\title{
Carbon Fiber Composite Cellular
}

\section{Structures}

\author{
A Dissertation
}

Presented to

the faculty of the School of Engineering and Applied Science

University of Virginia

In partial fulfillment

of the requirements of the degree

Doctor of Philosophy (Materials Science and Engineering)

by

Tochukwu George

May 2014 


\section{Approval Sheet}

The dissertation is submitted in partial fulfillment of the

requirements for the degree of

Doctor of Philosophy (Materials Science and Engineering)

Author

This dissertation has been read and approved by the examining committee:

Dissertation Advisor, Haydn N.G. Wadley (MSE)

Chairman, James M. Fitz-Gerald (MSE)

Dana Elzey (ES/MSE)

Sean Agnew (MSE)

Hilary Bart-Smith (MAE)

Accepted for the School of Engineering and Applied Science:

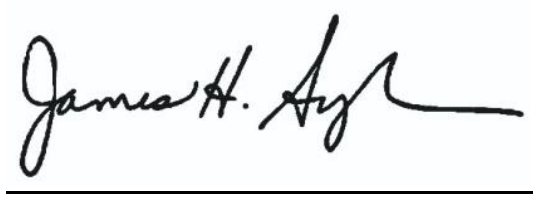

Dean, School of Engineering and Applied Science

May 2014 


\section{Abstract}

Ultralight sandwich panel structures which utilize light, stiff, and strong face sheets, and stiff, strong, compressible cellular cores have attracted significant research interest for use in aerospace and energy absorption applications. The cores of these sandwich structures are typically fabricated using high strength cellular materials, such as aluminum and titanium alloys, or polymer foams and honeycombs. However, for weight sensitive, ambient temperature applications, carbon fiber composites have emerged as a promising material due to its high specific strength and low density. Carbon fiber reinforced polymer (CFRP) composite cellular materials, when combined with structurally efficient sandwich panel designs, offer new opportunities for fabricating ultralight structures. This dissertation explores carbon fiber and carbon fiber hybrid sandwich panel design concepts, details the novel fabrication methods which have been developed for these structures, investigates the mechanical performance of the structures made using these approaches, and develops micromechanical models which predict the relationships between the mechanical properties of these structures and parent material properties, unit cell topology, and core density.

The dissertation develops four fabrication approaches for making carbon fiber and carbon fiber hybrid sandwich structures. These include a mechanical snap fitting and adhesive bonding method, a braided carbon fiber net method, a simplified linear braid approach, and a pultruded rod/linear tow technique. CFRP lattice structures made from carbon fiber laminates using a mechanical snap-fitting and adhesive bonding technique have been found to have high strength in through-thickness compression and in-plane 
shear. However, under compression or shear loading, these structures typically fail abruptly, exhibiting little ability to support load after initial strut failure. The trusses are also brittle, and absorb little energy during impact. The braided net fabrication approach utilizes a braided carbon fiber net within polymer and syntactic foams to form the core, woven carbon fiber face sheets, polymer and syntactic foam molds, and Kevlar stitching of core to the face sheets, followed by vacuum assisted resin transfer molding. This approach has facilitated the fabrication of empty lattice (truss and face sheet only) and hybrid core specimens ranging in density from $35-478 \mathrm{~kg} / \mathrm{m}^{3}$, and the mechanical performance of these structures characterized. The cellular structures made using this approach have been shown to have high compressive and shear strengths, as well as excellent energy absorption capacity. The linear braid method represents a simplified fabrication approach which utilizes linear braids to form the trusses within a hybrid cellular material. This method has facilitated the manufacture of hybrid cellular materials with a truss volume fraction ranging from $1.5-17.5 \%$ of the core. The fraction of the truss contained in the nodes is found to lead to saturation in core strength at higher densities. The pultruded rod/linear tow method enabled the fabrication of hybrid octet lattice structures which have a cell size independent of face sheet separation, and near isotropic properties. The micromechanical models presented in this dissertation offer a basis for materials design and analysis for composite structures for a wide variety of uses and applications. 


\section{Acknowledgements}

I would like to thank my advisor, Dr. Haydn Wadley, for his help and support throughout my years here at the University of Virginia. His mentorship and constructive advice has helped me become a much better scientist. I would also like to thank the

members of my committee, Dr. James Fitz-Gerald, Dr. Dana Elzey, Dr. Sean Agnew, and Dr. Hilary Bart-Smith for their valuable input, and for reviewing my dissertation. I would also like to acknowledge the members of the IPM research group here at the University of Virginia for numerous helpful discussions, suggestions, and assistance in the lab. Finally I want to thank my family for their love, support, and words of encouragement throughout my pursuit of my education. 


\section{Table of Contents}

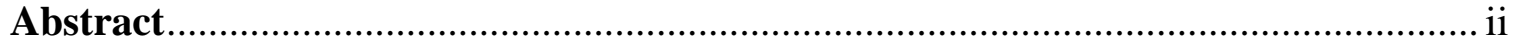

Acknowledgements ........................................................................................... iv

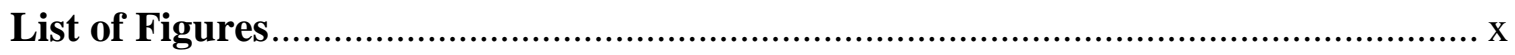

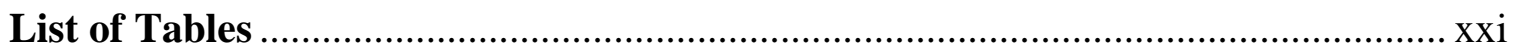

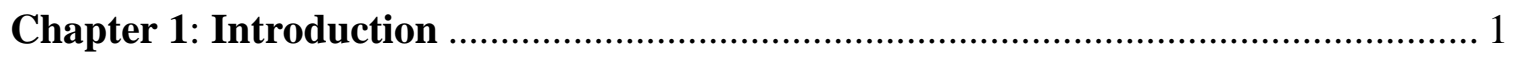

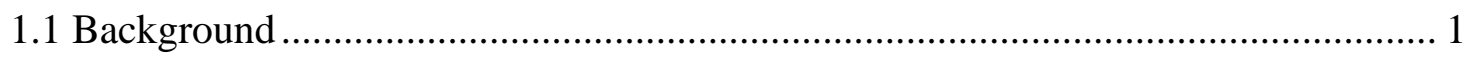

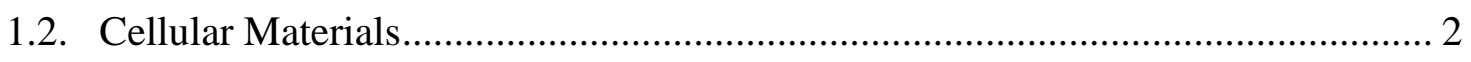

1.3 Stochastic Cellular materials............................................................................ 4

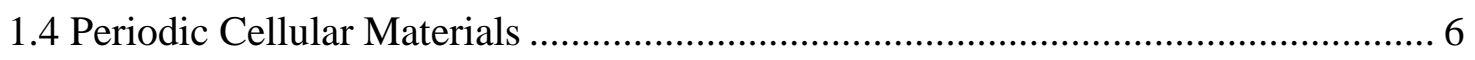

1.5 Carbon Fiber Truss Structures ...................................................................... 9

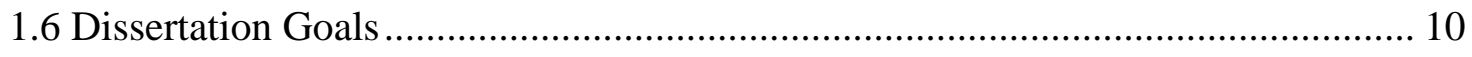

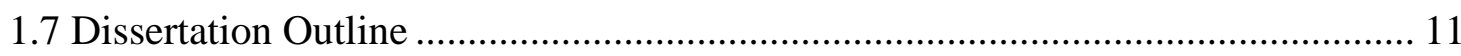

Chapter 2: Mechanical Properties of Periodic Cellular Materials.............................. 12

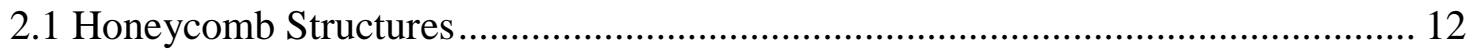

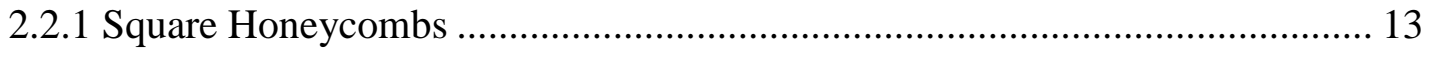

2.2.2 Hexagonal Honeycombs ....................................................................... 15

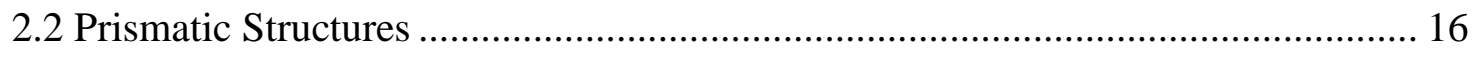

2.3 Lattice Truss Structures ................................................................................ 19

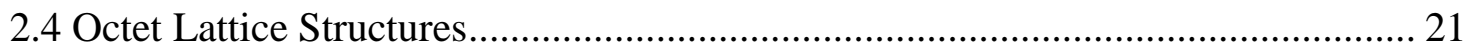


3.1 Materials and manufacture ......................................................................... 25

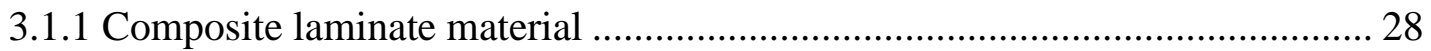

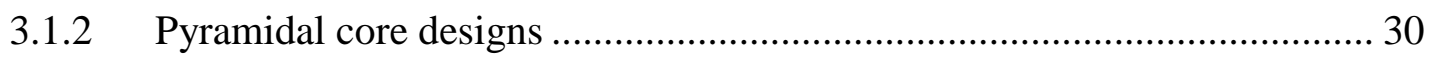

3.2 Measurements of the shear response of the pyramidal core............................ 31

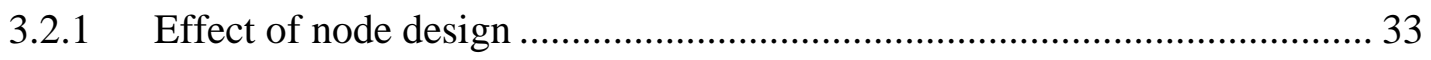

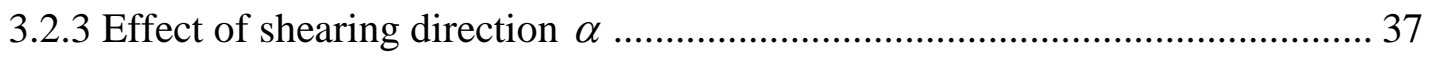

Chapter 4: Analytical Model of the Shear Response ............................................ 40

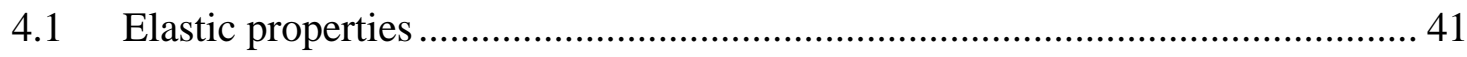

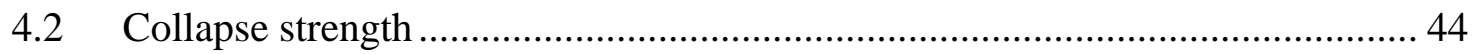

4.2.1 Plastic micro-buckling of the composite struts .......................................... 45

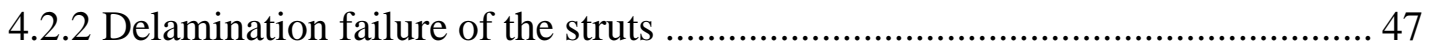

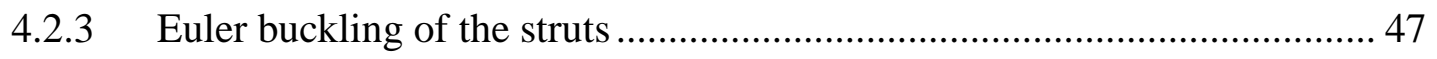

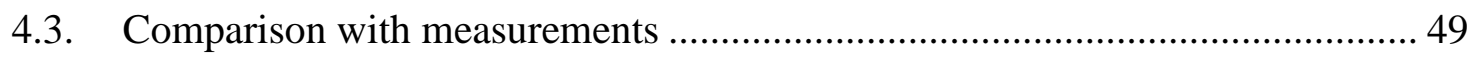

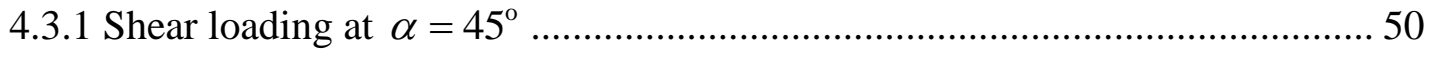

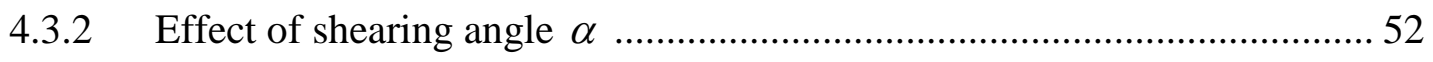

4.4. Comparison with competing materials .................................................. 52

4.5. Laminated structure advantages and limitations ..................................... 54

Chapter 5: Hybrid CFRP/Foam Structures …................................................. 55

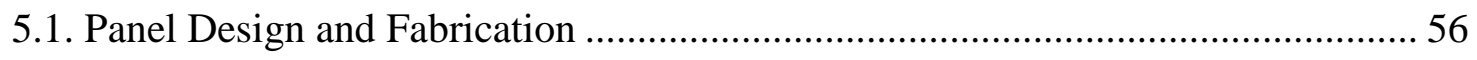




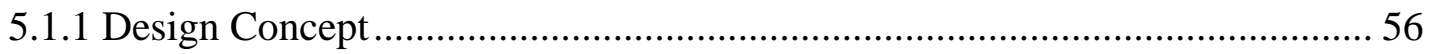

5.1.2 Braided net fabrication......................................................................... 58

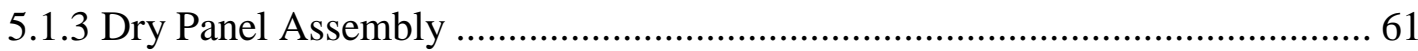

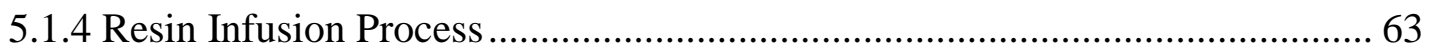

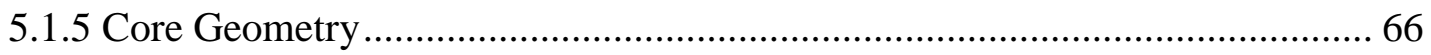

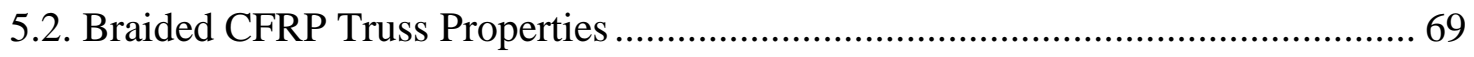

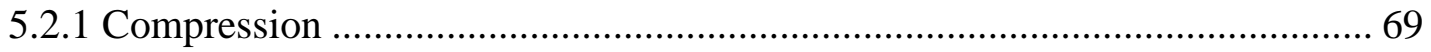

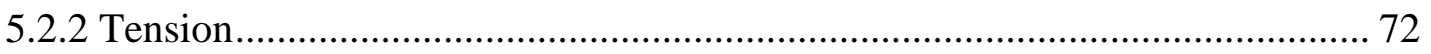

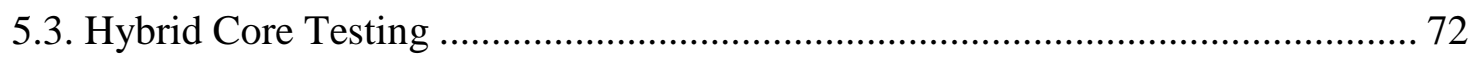

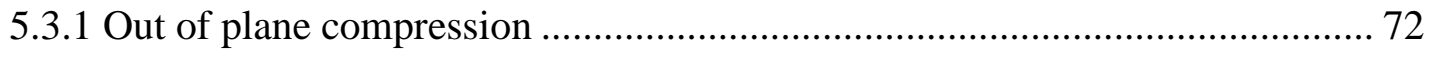

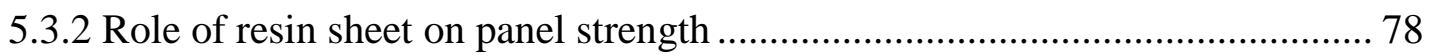

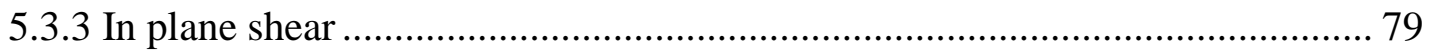

Chapter 6: Model of the braided CFRP/Foam Hybrid Core ................................. 82

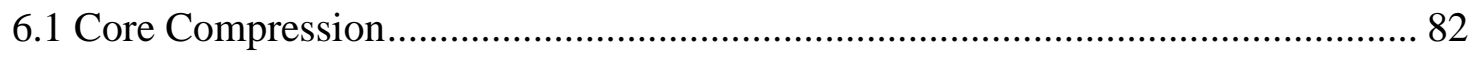

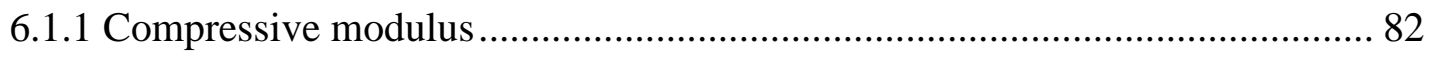

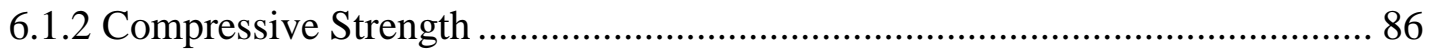

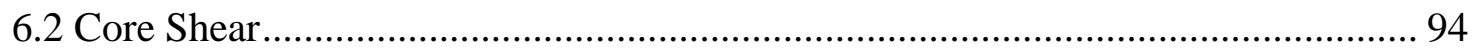

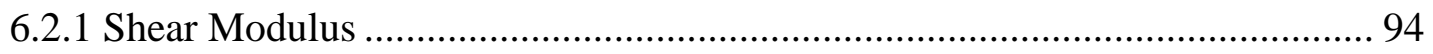

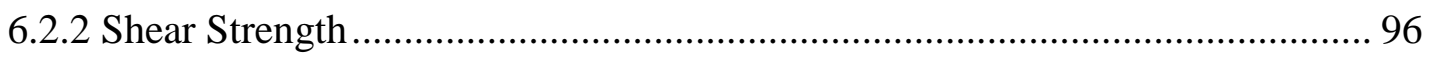

6.3 Energy Absorption ................................................................................ 98 


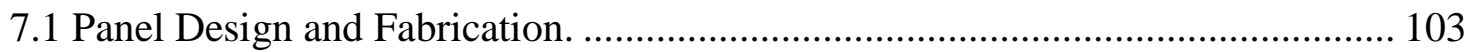

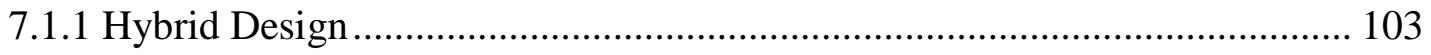

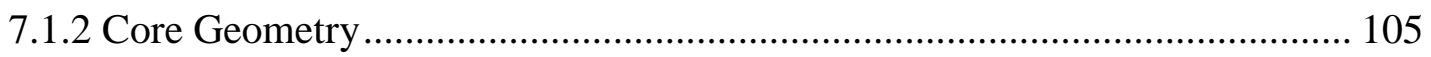

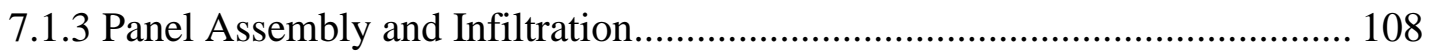

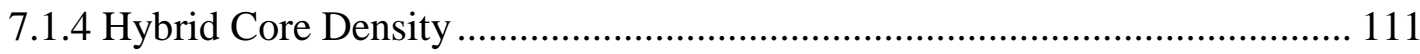

7.3. Mechanical Properties of Linear Braided Truss ............................................... 114

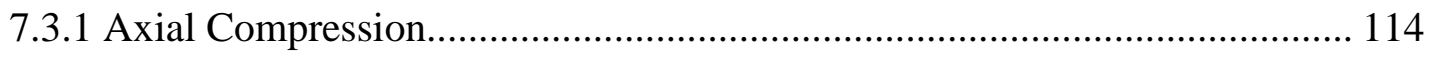

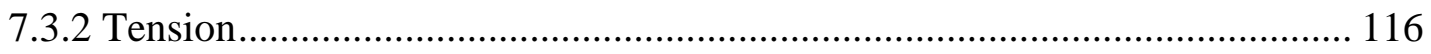

7.4. Hybrid Core Compression Response ……………………………................. 117

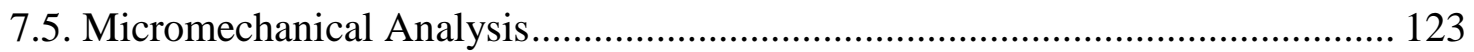

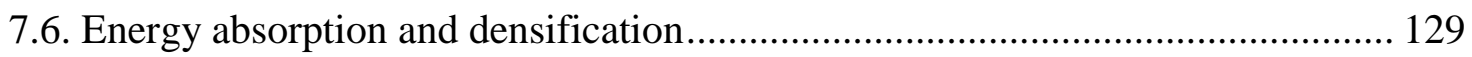

Chapter 8: Hybrid CFRP Octet Lattice Structures................................................ 134

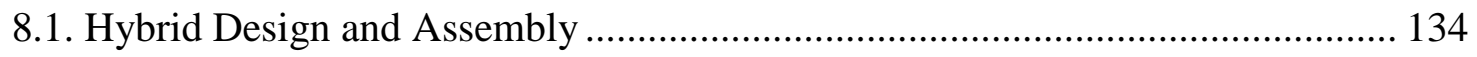

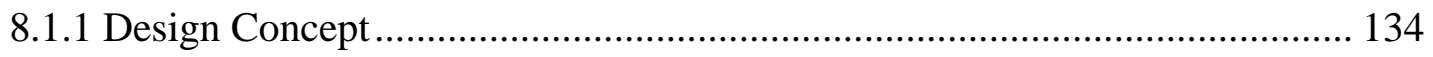

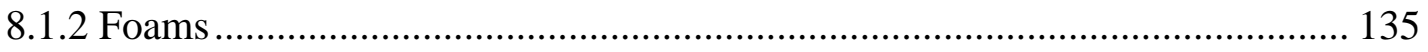

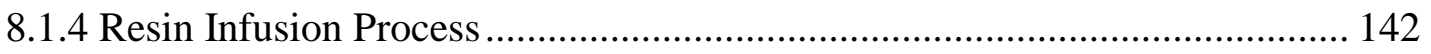

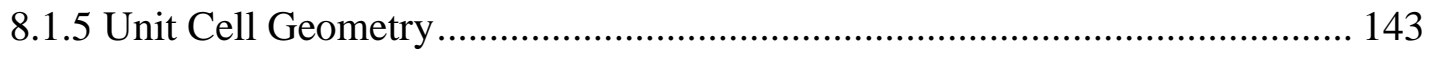

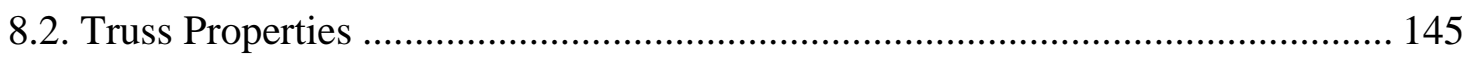

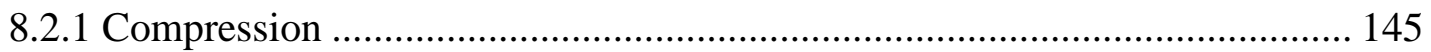




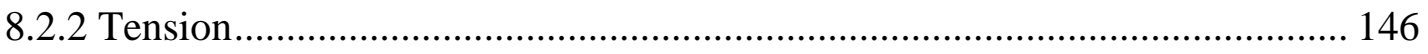

8.3. Hybrid Octet Lattice Response ................................................................... 148

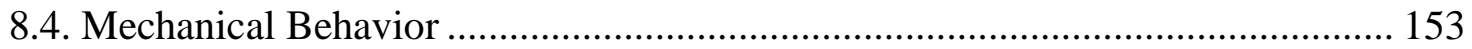

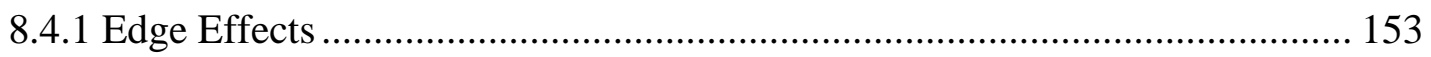

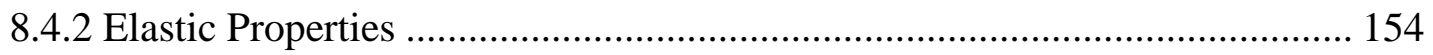

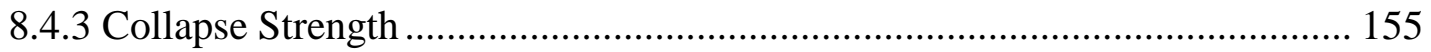

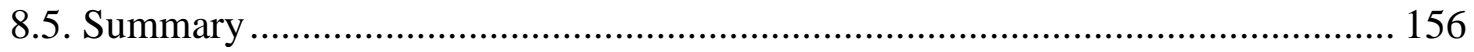

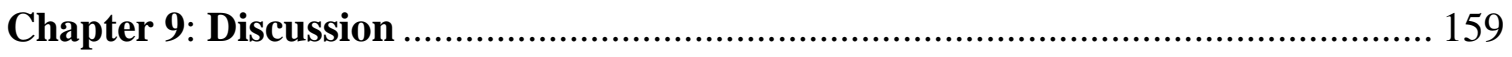

9.1 Stiffness, strength, and energy absorption.............................................. 159

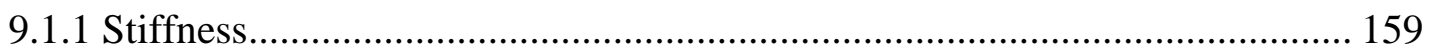

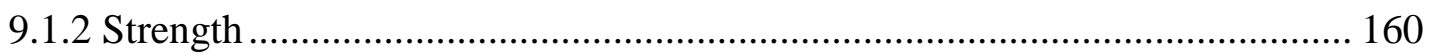

9.1.3 Hybrid Core Design for Energy Absorption Applications. .......................... 161

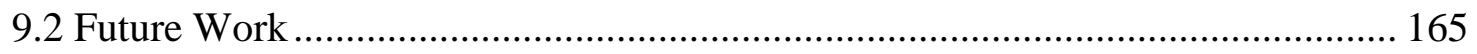

9.2.1 Application of fabrication methods to other composite materials ................ 165

9.2.2 Further exploration of hybrid octet lattices........................................... 167

9.2.3 Reduction of the the node saturation effect on core strength....................... 167

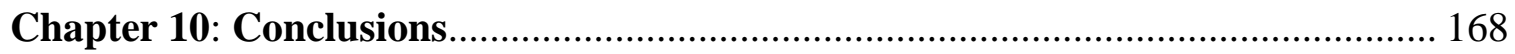

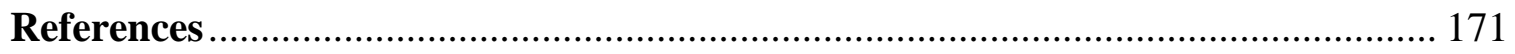




\section{List of Figures}

Figure 1.1: Modified Ashby Materials property charts for (a) compression and (b) shear loading. All "white spaces" are unachievable with current materials. The gaps identified by elliptical bubbles represent the region of opportunity investigated here 3

Figure 1.2: Different types of Stochastic Cellular materials 4

Figure 1.3: Schematic Illustration of Periodic Cellular Core Topologies...................8

Figure 2.1 Square honeycomb cell structure configured as a sandwich structure. .13

Figure 2.2 Hexagonal honeycomb cell structure configured as a sandwich structure.....16

Figure 2.3 Corrugated lattice sandwich structure unit cell dimensions .17

Figure 2.4 a) Pyramidal lattice unit cell with four struts, and b) tetrahedral lattice unit cell with three struts.

Figure 2.5: Octet lattice structure with circular cross-section trusses. The unit cell is shown in dashed lines .22

Figure 3.1: The fabrication route for making composite pyramidal lattice core sandwich panels. (a) Patterns were water jet cut from $0 / 90^{\circ}$ CFRP laminate sheets. The fiber directions are shown in this sketch. (b) Snap-fitting of the patterns to produce a pyramidal lattice. (c) Geometry of the truss pattern with relevant core design variables defined. (d) Fully assembled pyramidal lattice core sandwich panel. The composite face-sheets utilized cruciform shaped slots into which the pyramidal trusses were fitted and adhesively bonded. 
Figure 3.2: (a) Sketch of the unit cell of the pyramidal core with co-ordinate system marked. (b) Top-view of the unit cell indicating the direction of shear. (c) Photograph of the as-manufactured design 1 core $(\bar{\rho}=7 \%)$ in a sandwich panel structure

Figure 3.3: The measured uniaxial compressive stress versus strain response of the composite material along one of the fiber directions. Results for both a CLC test (a) and a column compression test (b) are shown. .30

Figure 3.4: Sketch of the single lap shear compression plate setup used to measure the shear response of the pyramidal cores.

Figure 3.5: (a) The measured shear stress $\tau$ versus strain $\gamma$ response $\left(\alpha=45^{\circ}\right)$ of the $\bar{\rho}=5 \%$ core for selected values of the node thickness $t_{0}$. (b) The measured peak strength as a function of node thickness for the $\bar{\rho}=5 \%$ core

Figure 3.6: (a) Photograph of the node failure mechanism in the $\bar{\rho}=5 \%$ core with $t_{0} / t=0.5$. (b) Sketch illustrating the node failure mechanism 35

Figure 3.7: The measured shear stress $\tau$ versus strain $\gamma$ response $\left(\alpha=45^{\circ}\right)$ of the pyramidal cores for selected values of the relative density $\bar{\rho}$ and node thickness $t_{0} / t=0.8$ .36

Figure 3.8: Photographs of specimens just after attainment of the peak load to illustrate the observed failure mechanisms. (b) Delamination observed in the $\bar{\rho}=5 \%$ specimen and (b) Euler buckling of the struts observed in the $\bar{\rho}=0.9 \%$ specimen..............38 
Figure 3.9: The measured shear stress $\tau$ versus strain $\gamma$ response of the $\bar{\rho}=5 \%$ pyramidal (node thickness $t_{0} / t=0.8$ ) for three selected loading directions $\alpha$. The analytical model predictions of the strength are also included

Figure 4.1: Schematics showing the fiber alignment on (a) the laminate sheets, and (b) The sandwich panel...................................................40

Figure 4.2 (a) Oblique and (b) planer views of the unit showing truss displacement due to an applied shear force .42

Figure 4.3: Predictions of the variation of the normalized peak strength $\tau_{p} /\left(\bar{\rho} \sigma_{\max }\right)$ with relative density $\bar{\rho}$ for three selected value of the normalized laminate modulus $\bar{E}_{s}$. The predictions assume that the node volume is negligible .48

Figure 4.4: Summary of the measured (a) unloading modulus and (b) peak strength as a function of relative density $\bar{\rho}$ for shear loading at $\alpha=45^{\circ}$ (node thickness $t_{0} / t=0.8$ ).

The lines are predictions of the micromechanical models $\left(t_{0} / t=0.8\right)$

Figure 4.5: An Ashby chart of (a) shear modulus and (b) shear strength as a function of density. In addition to a range of competing cellular materials, properties of the CFRP pyramidal cores measured in this study are included.

Figure 5.1: Hybrid composite core structure consisting of a braided CFRP pyramidal lattice with polymer foam inserts configured as the core of a sandwich panel with 3D woven carbon fiber composite face sheets.

Figure 5.2 (a) Schematic of 64 carrier single module used for 3D braiding. (b) Shape control in the 3BRAID® process; disengagement of fork gears (the color red indicates 
which gears are disengaged) causes the preform to split. Reengagement of the fork gears rejoins the preform

Figure 5.3 (a) Schematic illustration of the structure of the braided carbon fiber strut. (b) Photograph of a strut section of the braided net. c) The braided net structure, with unit cell shape and dimensions identified.

Figure 5.4: The hybrid CFRP pyramidal lattice core sandwich panel assembly sequence.

Figure 5.5: Setup used for the vacuum assisted epoxy resin infusion process

Figure 5.6 a) Photograph of a completed hybrid panel with CFRP pyramidal lattice and H250 Divinycell foam core. b) X-CT image of the sample shown in (a) revealing the interior structure of the finished panel with the foam digitally filtered out

Figure 5.7: Pyramidal CFRP unit cell with elliptical cross section trusses. The minor axis of the truss cross-section was perpendicular to the trapezoidal side face of the foam core (not shown) used to support the truss. .66

Figure 5.8: (a) Schematic illustration showing setup used to determine compressive strength of the braided composite strut, and (b) the orientation of the strut fracture surface. .69

Figure 5.9: (a) Axial compressive stress-strain response of a braided truss. (b) X-CT cross sectional image of a failed truss specimen showing damage initiating by microbuckling in one of the tows and shear crack propagation across the truss. (c) Axial

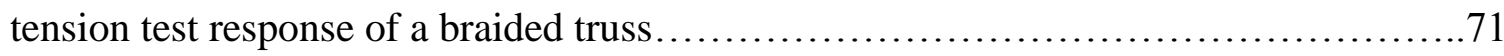


Figure 5.10: Compressive stress-strain responses for the hybrid CFRP pyramidal lattice/foam core panels constructed using Divinycell foams of different strengths (and densities). The response of just the foams is also shown......................... 73

Figure 5.11: Compressive stress-strain responses for the hybrid composite core panels constructed from syntactic foams of different strengths........................... 74

Figure 5.12: Reconstructed X-CT images of a hybrid composite panel with H250 foam core; (a) as fabricated and at various levels of strain (b-d). The trusses fail by microbuckling near the nodes while the resin sheets fail by buckling $\ldots \ldots \ldots \ldots \ldots \ldots . . .77$

Figure 5.13: The mechanical response of the empty CFRP lattice in (a) compression and (b) shear .78

Figure 5.14: Core compressive stress - strain response in the absence of a truss core for 3 foam densities. 80

Figure 5.15: Shear stress versus shear strain curves for the hybrid composite core sandwich panels made using (a) H80 and (b) H100 foam. The stress strain curves for the foams used are also shown. .81

Figure 6.1: Schematic and free body diagram showing deflection of the struts within the core upon application of a compressive load.

Figure 6.2: Barcharts showing: (a) the measured compressive modulus compared to the predicted modulus for select foam densities, and (b) the individual modulus components combined for the cumulative prediction $\left(\mathrm{E}_{\mathrm{core}}\right)$ for each of the specified core densities...87 
Figure 6.3: The micromechanical predictions for both the elastic and microbuckling strengths plotted as a function of the ellipticity ratio $d_{1} / d_{2}$. The compressive strength contribution of the CRFP lattice deduced by subtracting the contributions of the foam and resin sheets from the measured strength are also shown for the different foams (open

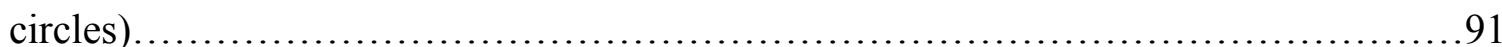

Figure 6.4: Barcharts showing (a) the measured core compressive strength compared to the micromechanical predictions, and (b) the individual components of the compressive strength prediction...................................................... 93

Figure 6.5: (a) The measured shear modulus of the composite cores compared to the micromechanical predictions. (b) The measured shear strength of the composite cores compared to the micromechanical predictions.

Figure 6.6: Material property chart showing (a) compressive strength, (b) shear strength, (c) compressive modulus and (d) shear modulus of CFRP honeycomb and pyramidal truss structures. The hybrid CFRP lattice/foam results of this study are also shown

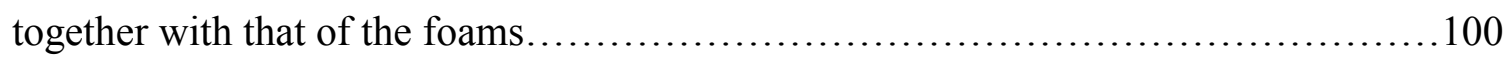

Figure 6.7: (a) The energy absorbed per unit volume and (b) energy absorbed per unit mass both plotted against density for low density cellular materials. The hybrid composites compare favorably with the other materials. 102

Figure 7.1: Hybrid composite core structure consisting linear carbon fiber braids with polymer foam inserts configured as the core of a sandwich panel with 3D woven carbon fiber composite face sheets..... 
Figure 7.2: Pyramidal CFRP unit cell with elliptical cross section trusses. Two linear braids are required to form each unit cell. 106

Figure 7.3: Illustrations of the structure of the linear braids together with photographs of the actual IM7 carbon fiber braids, containing (a) 8 tows, (b) 16 tows, (c) 32 tows, and (d) 64 tows. Each tow contained 12000 carbon fibers .107

Figure 7.4: The linear hybrid lattice core sandwich panel assembly sequence.... 109

Figure 7.5: (a) Structure of the 3 Weave face sheet (b) Reconstructed XCT showing top view of the 3 Weave material. 110

Figure 7.6: Reconstructed XCT images showing an untested specimen with the CFRP truss comprising: a) $1.5 \%$; b) $4 \%$, c) $7 \%$, and d) $17.5 \%$ of the core

Figure 7.7: Typical compressive stress-strain response for each of the infused linear braids tested. The specimens tested were all $20 \mathrm{~mm}$ in length, all fail by fiber microbuckling....

Figure 7.8: Typical tensile stress-strain responses for each of the linear braid composites tested.

Figure 7.9: Compressive stress-strain curves for hybrid linear braid panels

Figure 7.10: (a) The compressive modulus of the hybrid cores plotted against density (b) The compressive strength of the hybrid cores plotted against density.... . .120

Figure 7.11: Reconstructed X-CT images of a hybrid composite panel with 7\% truss volume fraction core (hybrid density of $364 \mathrm{~kg} / \mathrm{m}^{3}$ ): (a) as fabricated, (b) strained $10 \%$, 
and (c) strained 50\%. The trusses fail by fiber microbuckling at the circled locations and shear fracture near the nodes while the resin sheets fail by plastic buckling....

Figure 7.12: Reconstructed X-CT images of a hybrid composite panel with $7 \%$ truss volume fraction showing a longitudinal cross-sectional slice of the trusses (a) as fabricated state, (b) after $10 \%$ strain, and (c) after $50 \%$ strain 122

Figure 7.13: (a) The measured compressive modulus compared to that predicted for the different hybrid core densities, and (b) the individual modulus components of the prediction for each hybrid density 124

Figure 7.14: (a) The measured core compressive strength compared to micromechanical predictions, and (b) the components of the compressive strength prediction.... 127

Figure 7.15: The elastic buckling and microbuckling predictions CFRP lattice for the compared to the deduced empty lattice strength (total core strength minus foam and resin sheet) 128

Figure 7.16: Material property chart showing (a) compressive strength, and (b) compressive modulus of CFRP honeycomb and pyramidal truss structures. The linear braid results of this study are also shown together with that of the foams 130

Figure 7.17: (a) The energy absorbed per unit volume and (b) energy absorbed per unit mass both plotted against density for low density cellular materials. The hybrid composites made using linear braids compare favorably with the other materials 
Figure 7.18: (a) Schematic of densification model used to predict densification strain (b) The predicted densification strain compared to the measured values for the truss relative densities tested.

Figure 8.1: Schematic illustrating the design concept for the hybrid octet lattice .135

Figure 8.2: Schematic showing the fabrication procedure for the syntactic foams.... 136

Figure 8.3: Compressive stress-strain curves for $\mathrm{SC} 1$ syntactic foams with microsphere volume fractions of (a) $21 \%$, (b) $39 \%$, (c) $60 \%$, and (d) $65 \%$ respectively.....

Figure 8.4: SEM image showing the microstructure of the $65 \%$ glass volume fraction SC1 syntactic foam. 138

Figure 8.5: Schematic showing the assembly procedure for the hybrid octet lattice utilizing carbon fiber tows and a fiber threading method.

Figure 8.6: Schematic showing the assembly procedure for the hybrid octet lattice utilizing pultruded rods

Figure 8.7: The infiltration setup for the octet hybrid structures 142

Figure 8.8: Photographs showing finished octet hybrid specimens made using H80 and H250 divinycell polymer foams and linear tow trusses .143

Figure 8.9: Unit cell of the octet truss - foam hybrid .144

Figure 8.10: Stress-strain curves for the linear tow and pultruded rod trusses 147

Figure 8.11: XCT image slice showing mid-plane of failed linear tow truss. It can be seen that the truss fails by microbuckling of the fiber tows 
Figure 8.12: Compressive stress-strain responses for the hybrid octet lattices. Also shown are the stress-strain curves for the foams used, scaled by volume fraction within the hybrid.

Figure 8.13: Stress-strain curve for hybrid octet specimen made using pultruded rod trusses. Also shown are the stress-strain curves for the foam used, scaled by volume fraction within the hybrid

Figure 8.14: Reconstructed XCT images of a SC1 syntactic foam/ CFRP linear truss octet hybrid, showing one layer of cells a) as fabricated, and b) after loading to $10 \%$ strain. The failed trusses are shown in black circles.

Figure 8.15: a) The upper and lower predicted modulus compared to the measured modulus. b) The upper and lower predicted collapse strength compared to the measured values 157

Figure 8.16: Modified Ashby charts showing the (a) compressive moduli and (b) compressive strength of the hybrid octet structures compared to other CFRP structures. .158

Figure 9.1: Modified Ashby charts showing the (a) compressive moduli and (b) compressive strength of the structures developed in this dissertation, compared to other lattices/materials.

Figure 9.2: Modified Ashby charts showing the (a) compressive and (b) shear strengths of the structures developed in this dissertation, compared to other lattices/materials...163 
Figure 9.3: (a) Volumetric and (b) Gravimetric projections of the change in energy absorption of the linear braid hybrid cores for increases in the inclined strut angle $\omega$

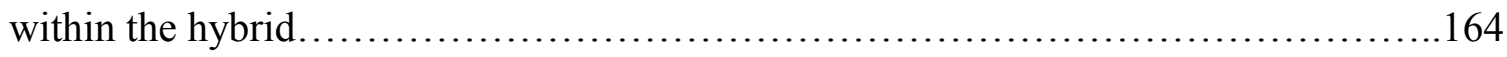

Figure 9.4: Proposed fabrication method for Nextel 610 metal matrix composites which mimics the linear braid fabrication procedure................................. 166 


\section{List of Tables}

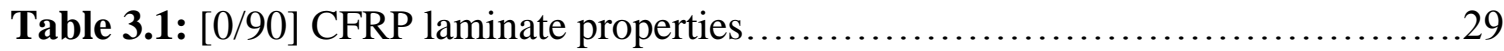

Table 3.2: Sandwich core parameters...................................... 31

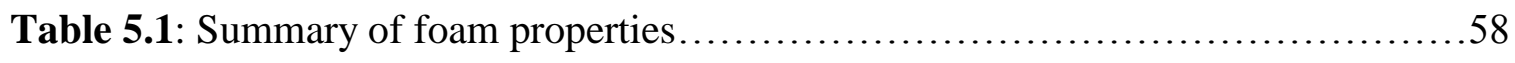

Table 5.2: Summary of core properties.................................... 75

Table 7.1: Summary of braid and CFRP strut properties.........................107

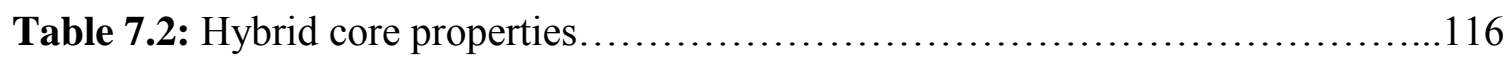

Table 8.1 CFRP truss properties........................................... 147

Table 8.2 Hybrid CFRP octet properties..................................151 


\section{Chapter 1}

\section{Introduction}

\subsection{Background}

The demand for ultralight sandwich structures for use in aerospace and other weight sensitive applications has resulted in the emergence of a number of light stiff and strong materials and structures. These include light metals such as magnesium, aluminum and titanium alloys, which have been successfully deployed ${ }^{1,2}$ and composites based on carbon fibers and polymeric light matrices ${ }^{3,4}$. Configured as sandwich panels consisting of pairs of light, stiff, strong faces separated by low density cores, these materials offer exceptional structural load support, especially in bending. The use of "cellular materials" concepts for the cores has grown in importance ${ }^{5}$, as these materials are used to provide a low weight means to separate face sheets, and get bending resistance. The strengths of cellular materials/structures depend on that of the materials from which they are made, and the cell topology. Aluminum alloy truss cores manufactured via an extrusion and folding process have been shown to have excellent mechanical properties in compression and shear ${ }^{6}$. Lattices made from higher specific strength titanium alloys have also been shown to have even higher compressive strengths and stiffnesses, and the potential for higher temperature aerospace applications ${ }^{7,8}$. However, for weight driven ambient temperature applications, carbon fiber materials have the highest specific strength, and when fabricated in the form of ultralight sandwich structures, appear to offer the best 
mechanical properties ${ }^{3,4}$. This dissertation explores methods for making ultralight carbon fiber and carbon fiber hybrid composites, to characterize their mechanical properties in compression and shear, and to establish mechanical property-composite-core topology relations for these panel structures. New CFRP cellular materials promise to expand the materials property space available to structural designers. This can be visualized using modified Ashby maps, which reveal the gaps that currently exist in the strength-density materials property space, as shown in Figure 1.1 for both compression and shear.

\subsection{Cellular Materials}

Many biologically synthesized materials such as bone, wood, coral, and bee honeycombs are highly porous, even though the presence of porosity in a material is usually viewed as undesirable, since they reduce the strength and stiffness of the material. However, these naturally occurring classes of materials, known as cellular solids, typically feature a hard outside layer and light weight interior with regularly spaced pores or voids ${ }^{9}$. They are therefore a form of sandwich construction and efficiently resist bending loads. These naturally occurring cellular materials have been in existence and utilized for thousands of years, but a better understanding regarding the mechanics of these structures has not been realized until more modern times. The presence of such structures in nature has spurred the design of artificial cellular structures with dense exterior layers which mimic the structural efficiency and load bearing capacity of these materials ${ }^{5}$. Cellular materials can be made with either stochastic or periodic topologies. In both cases, the cellular solids can be either open cell or closed cell. 

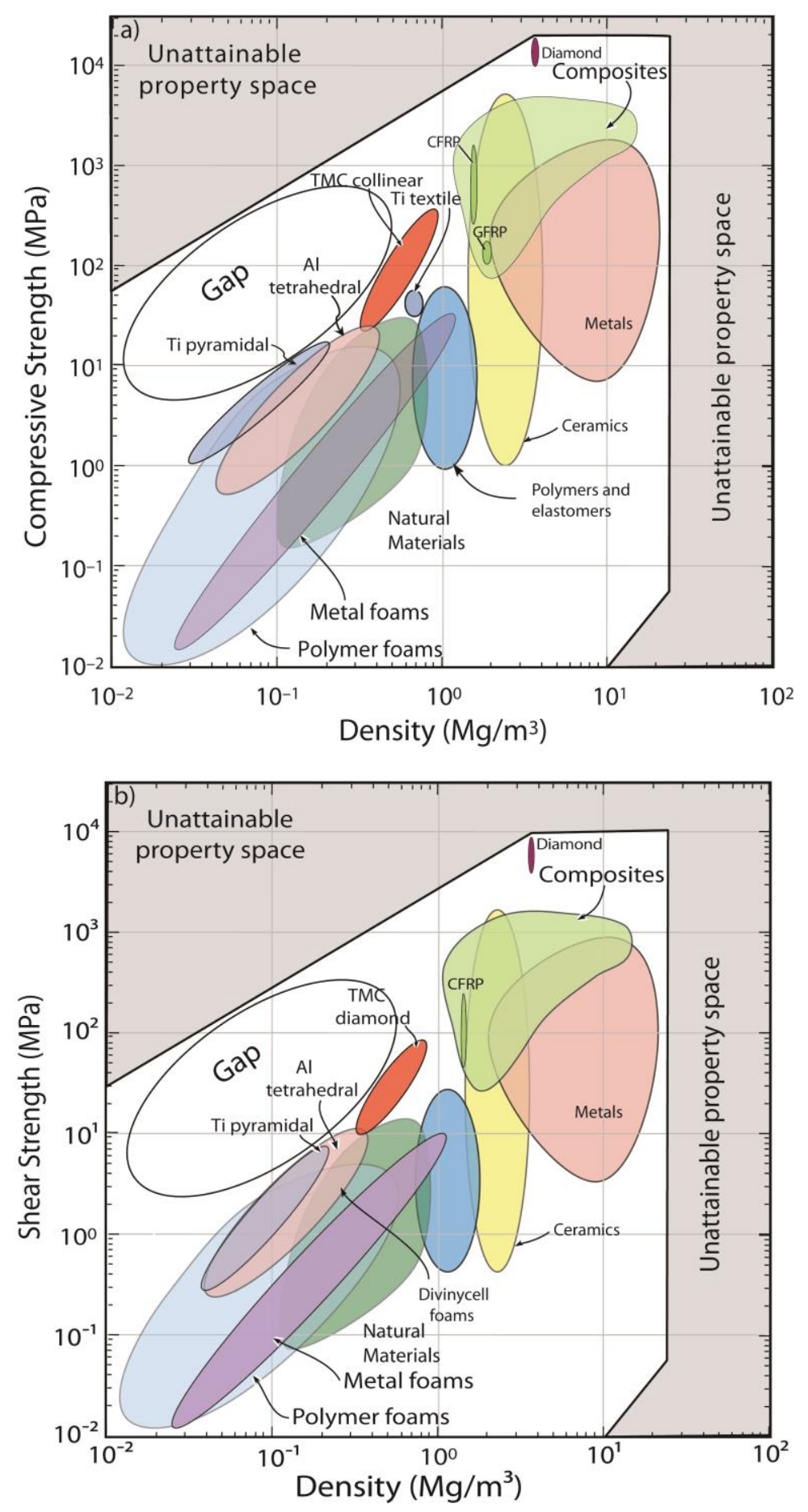

Figure 1.1: Modified Ashby ${ }^{10}$ materials property charts for (a) compression and (b) shear loading. All "white spaces" are unachievable with current materials. The gaps identified by elliptical bubbles represent the region of opportunity investigated here. 


\subsection{Stochastic Cellular materials}

The term "stochastic cellular materials" refers to a class of cellular materials which possess random architecture. This random architecture is observed in naturally occurring materials, such as wood, bone, cork, and coral, as well as artificially manufactured materials, such as metal and polymer foams, and features a random distribution of pores or voids within the structure. Figure 1.2 shows images of some commonly used stochastic cellular materials.

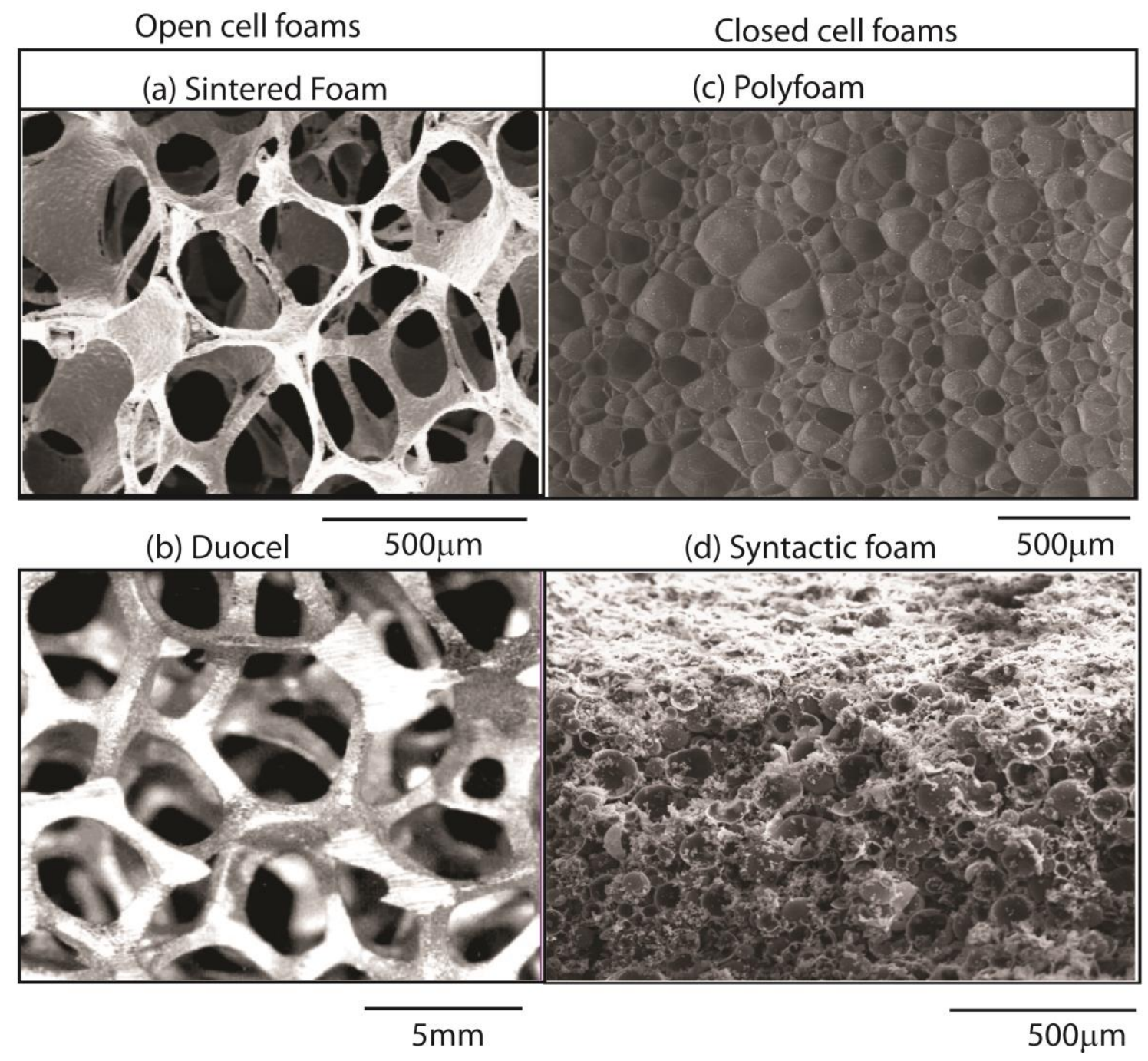

Figure 1.2: Different types of stochastic cellular materials ${ }^{11}$ 
There are two subclasses of stochastic cellular materials; open and closed cell structures. Closed cell structures (Figure $1.2 \mathrm{c}$ and d) feature membrane walls which completely surround the pores or voids, while open cell structures (Figure $1.2 \mathrm{a}$ and $\mathrm{b}$ ) are made of ligaments interconnected at nodes. The relative density of a cellular material $\bar{\rho}$ is the ratio of the density of the foam structure to that of the parent material. For both closed cell and open cell foams, the elastic modulus drops rapidly as the pore content is increased ( $\bar{\rho}$ decreases). Gibson and Ashby ${ }^{9}$ analyzed the micromechanical response of cellular materials and developed relations between foam properties, parent material properties, and the relative density of the foam. For open cell foams, they show that:

$$
\frac{E^{*}}{E_{s}} \approx \bar{\rho}^{2}
$$

where $E^{*}$ is the modulus of the foam, $E_{s}$ is the value of the Young's modulus of the solid parent material. Similarly, for closed cell foams,

$$
\frac{E^{*}}{E_{s}} \approx \phi^{2} \bar{\rho}^{2}+(1-\phi) \bar{\rho}
$$

where $\phi$ is the fraction of solid in the edges ${ }^{9}{ }^{12}$. The compressive strength of a foam also drops rapidly with decreasing relative density. Gibson and Ashby ${ }^{9}$ found that for open cell foams, the strength can be approximated by:

$$
\frac{\sigma_{p l}^{*}}{\sigma_{y s}} \approx 0.3 \bar{\rho}^{3 / 2}
$$


where $\sigma_{p l}^{*}$ is the plastic collapse strength of the foam, and $\sigma_{y s}$ is the yield strength of the cell wall material. Similarly, for closed cell foams,

$$
\frac{\sigma_{p l}^{*}}{\sigma_{y s}} \approx 0.3(\phi \bar{\rho})^{3 / 2}+0.4(1-\phi) \bar{\rho}
$$

Foam structures made from polymers, metals, and ceramics have been extensively investigated at the fundamental level ${ }^{12,13,14}$. The manufacturing process for making stochastic foams often results in a non-uniform cell structure, with relatively large numbers of defects, and sometimes random and non-identical curvature of the cell walls. As a result of this heterogeneous structure, the strength and stiffness can often vary from place to place within the cellular structure. While both polymeric and metal foams have been used as cores within sandwich structures, their low strength and modulus are not optimal for most applications ${ }^{15}$.

\subsection{Periodic Cellular Materials}

Periodic cellular materials are ordered cellular structures. These materials are typically highly porous structures with $20 \%$ or less of their interior volume occupied by solid $^{16}$. They are often stretch dominated structures whose strengths increase linearly with the relative density ${ }^{16,17,18}$. Periodic cellular structures feature repeating unit cells which have a periodicity in three dimensions. The three main types of periodic topologies, namely prismatic, honeycomb, and truss cellular structures. Prismatic cores are usually made from plates or sheets, and have open cells in one direction and closed cells in the other two directions (see Figure 1.3). There are three main types of prismatic topology, namely triangular, diamond, and nav-truss. Prismatic cores are often manufactured by a 
bending or plate folding process, or by an extrusion technique ${ }^{16,19}$. Since the cells are only open in one direction, prismatic topologies are often used in fluid flow applications to enable fluid flow in one direction but not others ${ }^{16}$. Honeycomb cores are closed cell structures comprised of plate or sheet elements that form the edges of the unit cell. Honeycombs can be made in triangular, hexagonal or square shapes (see Figure 1.3), or slight variations of these shapes. Honeycomb structures can be manufactured by a slotting process, or by a corrugation and stacking process ${ }^{20,21}$. The slotting process can be used to make square and even triangular shaped cells which are less anisotropic than hexagonal honeycombs, and more efficiently resist in-plane stretching. Honeycomb structures offer superior mechanical performance when compared to stochastic foams, but have limited multifunctional capability due to the closed cell nature ${ }^{16,22}$. Truss cores are fully open cellular structures which are made from slender beams (struts or trusses). These structures can be made with a number of cross-sectional shapes, including square, rectangular or tubular. Examples of truss core topologies include pyramidal, tetrahedral, 3-d kagome, square collinear, octet, and other variations of these shapes. Truss cores feature ligaments which are interconnected at node points, and in some cases form a continuous network. A number of fabrication techniques for truss cores have been developed, including a perforation and folding method ${ }^{23}$, which is suited for metal alloys, investment casting ${ }^{24}$, hollow tube layup ${ }^{25}$, and mechanical snap fitting ${ }^{26}$. Octet lattice topologies have the added advantage of near-isotropic properties, i.e., the mechanical properties are similar when tested in different orientations ${ }^{27}$. 


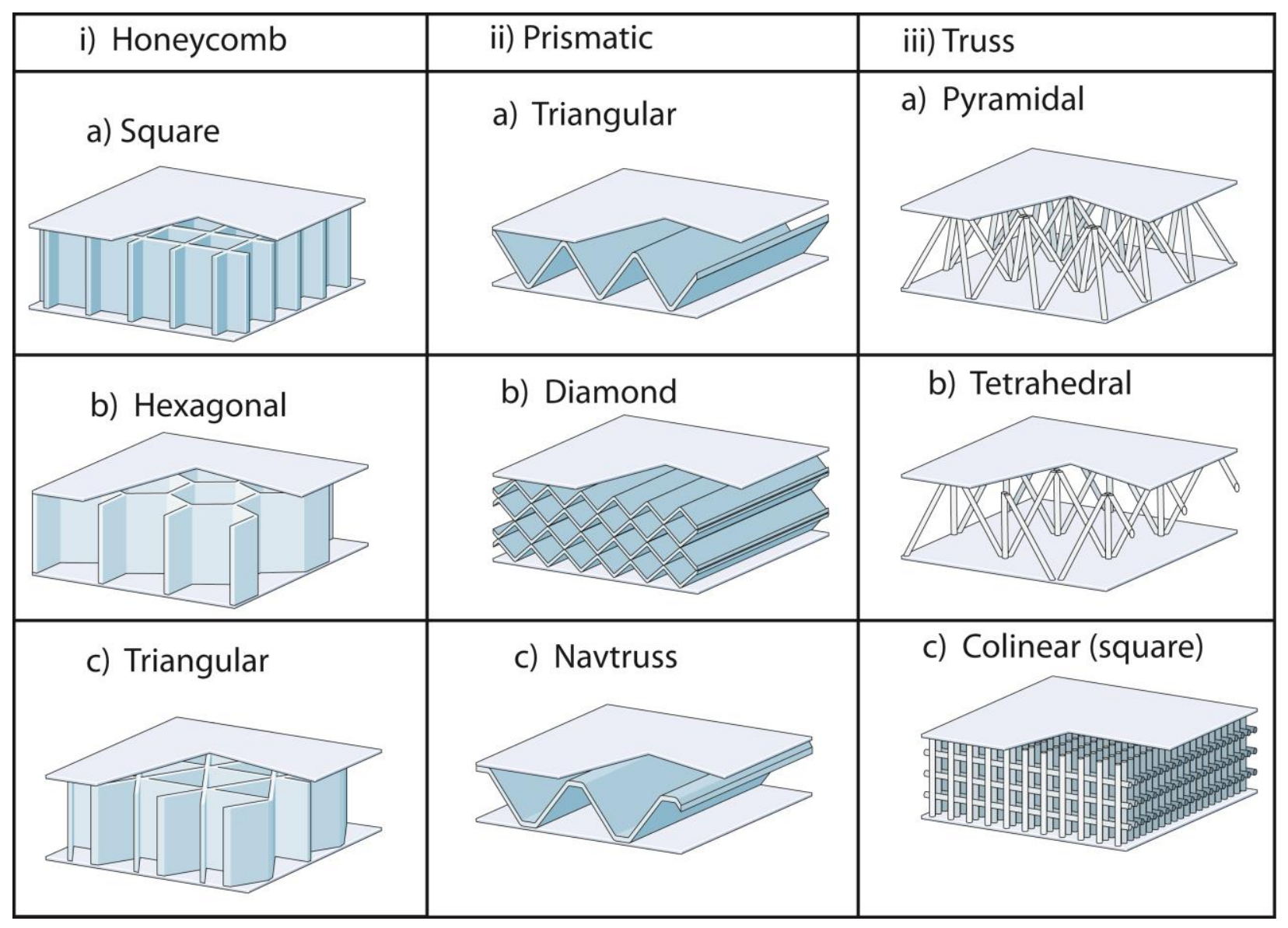

Figure 1.3: Schematic Illustration of Periodic Cellular Core Topologies (modified from references 16 and 28 )

Cellular structures can provide several simultaneous functionalities. For example, closed cell foam cored sandwich panels can support bending loads and mitigate impact loads well, while open celled systems can provide cross-flow heat exchange in addition to structural load support ${ }^{29,30,31}$. Lattice truss cores are usually stretch dominated, due to the fact that the core ligaments experience axial stress, i.e., either compression or tension, when the core is loaded in compression, shear, or bending. The strength of these lattice structures scales linearly with the relative density $\bar{\rho}$ of the cellular structure ${ }^{16,24}$ until buckling of the struts occurThe mechanical performance of lattice truss cores within 
sandwich panels is thus superior to that of stochastic foams that deform by bending, as can be seen in Figure 1.1. As a result, lattice truss cores are widely preferred for use in sandwich cores over stochastic foams ${ }^{24}$.

It has been shown that the strength of sandwich panels with lattice cores is dependent on the properties of the material from which the lattice is made, as well as its topology ${ }^{16,18,24}$. It thus follows that increasing the mechanical properties of these structures would require use of high strength materials to fabricate the lattice cores.

\subsection{Carbon Fiber Truss Structures}

The use of carbon fiber materials within the core of sandwich structures has generated significant interest in recent years. Carbon fiber honeycomb sandwich structures made from 0/90 carbon fiber reinforced polymer (CFRP) laminates by slotting and adhesive bonding have been shown to have high compressive strengths ${ }^{26}$. Carbon fiber truss structures have also been made by the hot-press molding of carbon fiber prepreg materials, and also perform well in compression ${ }^{32,33}$. Recently, a mechanical snapfitting method has been developed for manufacturing carbon fiber pyramidal truss cores from 0/90 CFRP laminates, and has been shown to produce sandwich panels with very high compressive strengths at low densities ${ }^{4}$. Work presented in Chapters 3 and 4 explores the in-plane shear response of cellular structures made in this way. The high specific stiffness and strength, along with the low density of carbon fiber materials have made them attractive for sandwich panel construction.

Carbon fiber laminates in general are limited in strength in axial compression due to brooming, delamination, and microbuckling, and fail by fiber fracture in tension. All 
the trusses in a carbon fiber lattice made using laminates experience a compressive load when the lattice is loaded in through-thickness compression ${ }^{3}$. When loaded in in-plane shear, half the trusses experience a compressive load, while the other half experiences a tensile load ${ }^{34}$. The compressive strength of carbon fiber lattices is important to optimize, and can be enhanced by eliminating low stress failure modes. In addition, many CFRP structures typically fail catastrophically, with little ability to support load after the initial strut failure. They would therefore not be suitable for absorbing the kinetic energy of an impact; something that polymer and metal foams do well by plastic work (dissipation). Thus, increasing the energy absorption capability of CFRP lattice structures is also of great interest. The use of braided carbon fiber material to create the trusses of the lattice might potentially help suppress delamination, and result in higher sandwich panel strengths. The use of a braided CFRP truss, in combination with light weight, high performance foams, has the potential to create efficient, light weight and high strength hybrid carbon fiber composites.

\subsection{Dissertation Goals}

The primary objectives of this dissertation are to: (i) investigate the possibility of making sandwich structures and sandwich structure hybrids using carbon fiber reinforced polymer (CFRP) materials with low density and high moduli and strength; (ii) develop methods for manufacturing these carbon fiber and carbon fiber hybrid sandwich structures; (iii) explore and characterize the mechanical properties as a function of unit cell parameters, core topology, density, and constituent material properties; and (iv) to develop micromechanics-based models which predict the mechanical behavior of these structures. 


\subsection{Dissertation Outline}

The remainder of this dissertation is organized as follows: Chapter 2 reviews the mechanical properties of periodic cellular materials, including truss structures. Chapter 3 describes the fabrication technique and materials used to manufacture CFRP sandwich structures from laminates, as well as the testing procedures and mechanical behavior of these structures in in-plane shear. Chapter 4 develops a micromechanical model for the mechanical performance of the laminate structures in shear. Chapter 5 describes a design concept, materials, and manufacture of CFRP/Foam hybrid structures based upon use of a braided net. Chapter 6 presents a micromechanics model for the mechanical behavior of these CFRP/Foam hybrid structures in through thickness compression and in-plane shear. Chapter 7 describes a simplified fabrication technique for making CFRP/Foam hybrid structures using a linear braid, and investigates the consequences of increasing the volume fraction of CFRP trusses within the unit cell. Chapter 8 describes the design and manufacture of a CFPR/Foam hybrid octet lattice, and characterizes the mechanical properties. Chapter 9 then discusses the significance of these results in the context of the goals of the dissertation, and several suggestions for future work. Finally, Chapter 10 summarizes the conclusions of the dissertation. 


\section{Chapter 2}

\section{Mechanical Properties of Periodic Cellular}

\section{Materials}

As discussed in Chapter 1, stretch dominated periodic cellular materials have a highly ordered architecture, which in turn affects the mechanical performance of sandwich structures which have periodic cellular cores. This Chapter reviews the relationships between the mechanical properties of the periodic cellular structures and that of the parent materials used to construct them. The periodic structures examined include honeycomb, prismatic, and lattice truss cellular structures.

\subsection{Honeycomb Structures}

There are three main kinds of honeycomb structures, namely square honeycombs, hexagonal honeycombs, and triangular honeycombs. Triangular honeycombs are difficult to make, and not widely used. A number of methods have been devised over the years for fabricating square and hexagonal honeycomb structures, and this includes a slotting and brazing approach ${ }^{21,35}$, slotting and adhesive bonding ${ }^{36}$, and by stacking of corrugated layers $^{37}$. The relationships between the mechanical properties of square and hexagonal honeycomb topologies and that of the parent material is examined below. 


\subsubsection{Square Honeycombs}

Figure 2.1 shows an illustration of the square honeycomb cell topology. The relative density of a cellular structure can be determined by calculating the volume of truss contained within the unit cell, and then dividing that by the volume of the unit cell. For the square honeycomb for instance, the total volume of truss in the unit cell is equal to $2 t l H$, while the volume of the unit cell is $l^{2} H$. Thus, the relative density is given by ${ }^{21,36}$ :

$$
\bar{\rho}=\frac{2 t}{l}
$$

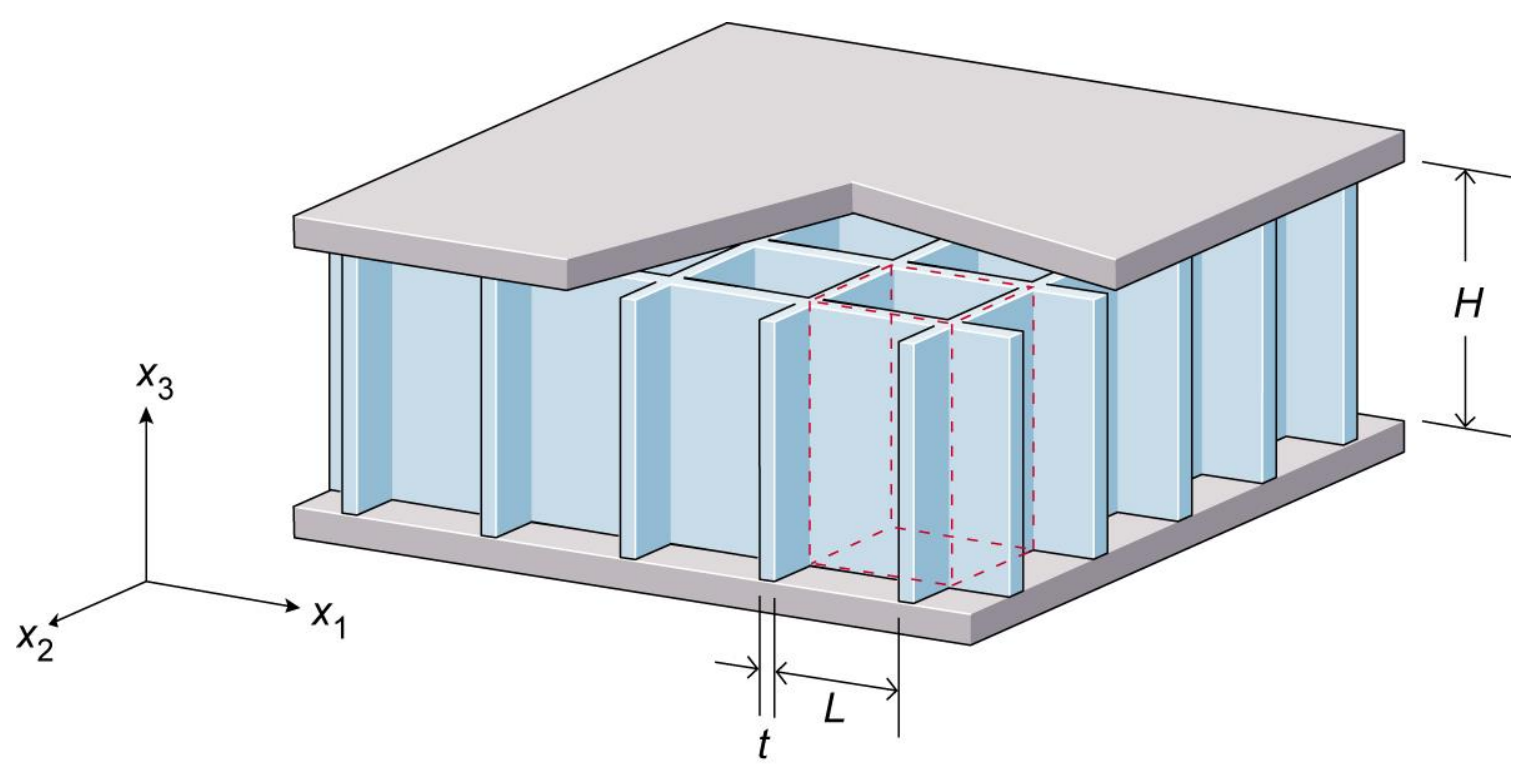

Figure 2.1 Square honeycomb cell structure configured as a sandwich structure. 
where $t$ is the cell wall thickness and $l$ is the wall spacing, as shown in Figure 2.1. The Young's modulus of the square honeycombs is simply the Young's modulus of the cell wall parent material, scaled by the relative density, expressed $\operatorname{as}^{21,36}$ :

$$
E=\bar{\rho} E_{s}
$$

where $E_{s}$ is the elastic modulus of the cell wall parent material. Equation 2.2 is also equivalent to the elastic modulus of the cell wall material multiplied by the ratio of the area of the truss ligaments to that of the unit cell. In cases where the cell wall material has anisotropic elastic properties, the elastic modulus of the parent material along the direction applied force (parallel to the cell walls) is used.

The square honeycomb lattice typically fails by elastic or plastic buckling of the cell walls. The plastic buckling stress for the honeycomb lattice can similarly be expressed as the plastic collapse strength of the cell wall material scaled by the relative density:

$$
\sigma_{b}=\rho \sigma_{p l}
$$

where $\sigma_{p l}$ is the plastic buckling strength of the cell wall material ${ }^{21,36}$ For cases where the lattice fails by elastic buckling, it is necessary to determine the critical buckling stress for cell wall material, $P_{e l}$. This is dependent on the geometry of the cell walls (rectangular), as well as the elastic modulus of the cell wall material. The critical buckling stress required for elastic buckling of the cells $P_{e l}$, is given by ${ }^{21,36}$ :

$$
P_{e l}=\frac{K \pi^{2}}{6(1-v)} G \frac{t^{3}}{L}
$$


where $K$ is the buckling coefficient, $G$ is the elastic shear modulus of the cell wall parent material, and $v$ is the Poisson's ratio. The buckling coefficient $\mathrm{K}$ is determined based on the boundary conditions at the top and bottom edges of the cell. The elastic buckling strength of the square honeycomb in terms of the critical buckling stress can then be expressed as:

$$
\sigma_{e l}=\frac{P_{e l} \bar{\rho}}{t}
$$

The peak strength of the square honeycomb would be determined by the failure mode which is attained at the lowest load level.

\subsubsection{Hexagonal Honeycombs}

Hexagonal honeycomb lattices have six sided unit cells, as shown in Figure 2.3. Regular hexagonal honeycombs have an angle of $\theta=120^{\circ}$ between the cell walls, and cell wall height $h$ is equal to the cell wall length $L$. Using trigonometry, we can calculate the volume of truss, and the volume of the unit cell, and it can be seen that the relative density of the hexagonal honeycomb can be written as ${ }^{38,39}$ :

$$
\rho=\frac{2}{\sqrt{3}} \frac{t}{L}
$$

The linear elastic properties of hexagonal honeycomb structures are governed by the bending of the cell walls, which can be modeled as beams subjected to an axial load ${ }^{38}$. For a through-thickness compressive load, the elastic modulus and strength of the core are well estimated by Equations 2.2 and 2.3, i.e, the parent material properties scaled by the relative density, in a similar manner to square honeycombs. 


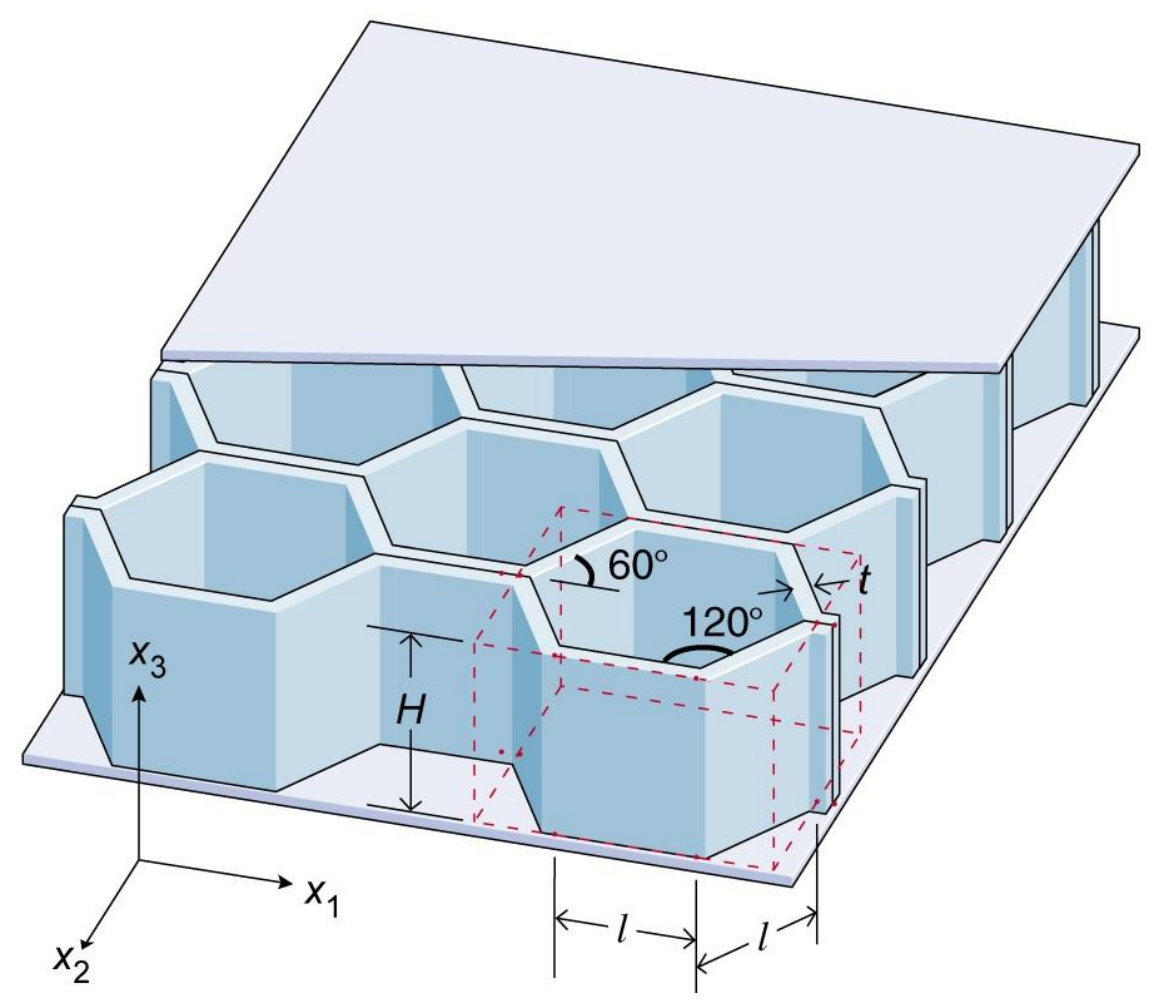

Figure 2.2 Hexagonal honeycomb cell structure configured as a sandwich structure.

\subsection{Prismatic Structures}

Prismatic cellular structures typically have open cells along one dimension, and closed cells in the other dimensions. The open cells run along the entire length or width of the specimen, as shown in Figure 1.3. The most common prismatic core structures are typically corrugated, and include the triangular, diamond, and nav-truss topologies. A number of fabrication methods are commonly used to create corrugated prismatic cores, including slotting and electro-discharge machining ${ }^{19}$, a folding and brazing $\operatorname{method}^{40}$, and an extrusion and welding approach ${ }^{41}$. Figure 2.1 shows an illustration of a typical one- 
layer corrugated core, including the relevant dimensional parameters of the core, namely the thickness of

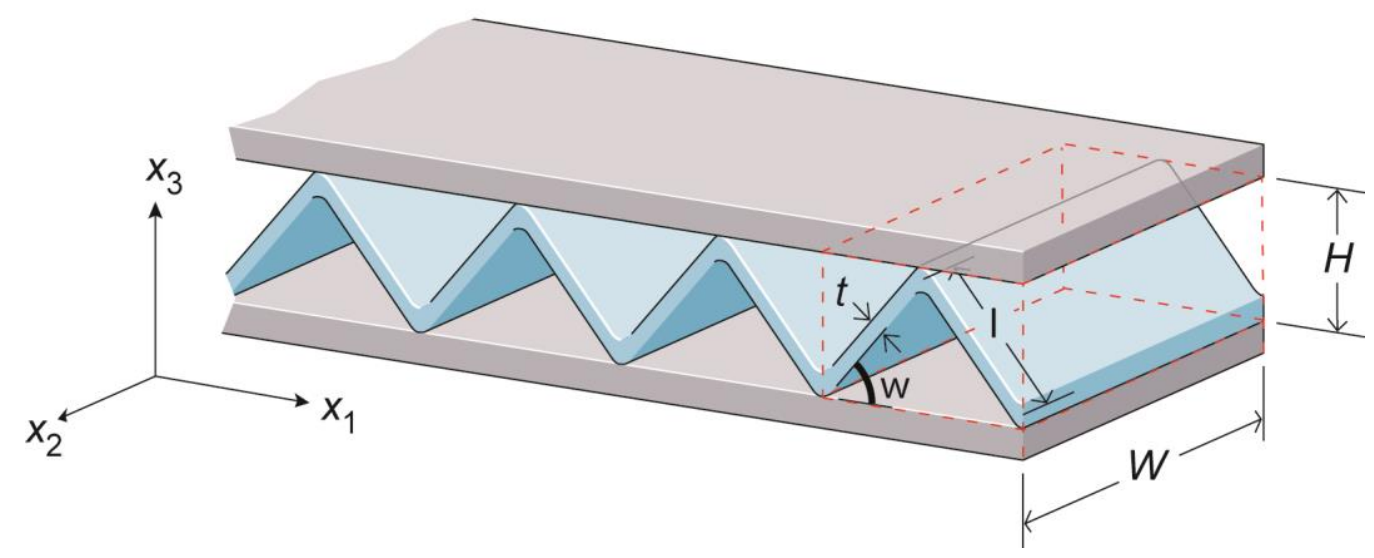

Figure 2.3 Corrugated lattice sandwich structure unit cell dimensions.

the corrugations, $t$, the truss length $l$, and the angle of inclsination of the corrugations $\omega$. The relative density of a corrugated structure is again calculated from the total truss volume divided by the volume of the unit cell. The volume of truss within the unit cell in this case would be equivalent to $2 t l W$. The volume of the unit cell can be obtained in terms of $t, l$, and $\omega$ using trigonometry, and is equivalent to $(l \sin \omega)(2 l \cos \omega) W$. Thus, the relative density of the structure can be expressed as:

$$
\bar{\rho}=\frac{2 t}{l \sin 2 \omega}
$$

where the angle of inclination of the corrugations, $\omega$, typically lies between $45^{\circ}$ and $60^{\circ}$. The truss angle $\omega$ determines the modulus and strength in out of plane compression $\left(x_{3}\right.$ direction) and in-plane shear ( $x_{1}$ direction) For an applied through thickness compressive 
load, contribution to the overall stiffness due to bending is negligible compared to the contribution due to stretching. The elastic modulus of the core in the through-thickness direction, $E_{L}$, can be determined by multiplying the elastic modulus of the parent material by the relative density, and a geometric factor. This geometric factor is determined by a trigonometric resolution of forces, depending on the topology of the lattice. For a core where all the truss ligaments are vertical, and $\omega$ is $90^{\circ}$ (for example, the I-core), this geometric factor is 1 (This is also true for honeycombs as well). For corrugated lattices, this geometric factor is $\sin ^{4} \omega^{19,40}$. The elastic modulus of the corrugation when loaded in the $x_{3}$ can then be expressed as:

$$
E_{3}=E_{s} \bar{\rho} \sin ^{4} \omega
$$

where $E_{s}$ is the Young's modulus of the parent material. Using the same method, it can be determined that for an applied in-plane shear force, the effective shear modulus of the corrugated core, $G_{l}$, can be expressed as follows ${ }^{19}$ :

$$
G_{1}=\frac{E_{s} \bar{\rho} \sin ^{2} 2 \omega}{4}
$$

The peak strength of the core when a through-thickness compressive force is applied would also depend on the strength of the constituent trusses, the relative density, and the geometric factor. The geometric factor has been determined to be $\sin ^{2} \omega$ for a compressive force $^{19}$. Therefore, the predicted peak strength of the corrugated core, $\sigma_{3}$, would be given by:

$$
\sigma_{3}=\sigma_{c} \bar{\rho} \sin ^{2} \omega
$$


where $\sigma_{c}$ is the critical buckling strength of the constituent trusses. These trusses buckle either plastically or elastically based on the slenderness ratio, $t / l$. Using the same methodology, the transverse shear strength of the core, $\sigma_{1}$, after resolution of forces, would be equivalent to ${ }^{19,40}$ :

$$
\sigma_{1}=\sigma_{c} \frac{\bar{\rho}}{2} \sin 2 \omega
$$

The equations reviewed above allow for a determination of the expected mechanical performance of the corrugated structure prior to manufacture.

\subsection{Lattice Truss Structures}

Lattice truss structures typically comprise of slender trusses separated by face sheets. The truss-face sheet interface (nodes) facilitates the transfer of an applied load, typically resulting in the individual truss ligaments deforming by stretching, and the individual ligaments experience a tensile or compressive force. The most common truss core topologies are pyramidal, tetrahedral, octet, and collinear lattices. A number of techniques have been devised for fabricating these topologies, including perforation and

folding $^{42},{ }^{43}$, investment casting ${ }^{44}$, and collinear lay-up and brazing ${ }^{25}$. Figure 2.4 shows an illustration of the unit cell for both the pyramidal and tetrahedral core. The relative densities for the tetrahedral core and the pyramidal core using the same methodology as of dividing truss volume by unit cell volume, can be expressed by the equations below:

$$
\bar{\rho}=\frac{2 \pi}{\sqrt{3}} \frac{1}{\cos ^{2} \omega \sin \omega}\left(\frac{r}{l}\right)^{2}
$$




$$
\bar{\rho}=\frac{2 \pi}{\cos ^{2} \omega \sin \omega}\left(\frac{r}{l}\right)^{2}
$$

where $r, l$, and $\omega$ are the radius, length, and angle of inclination of the trusses respectively.

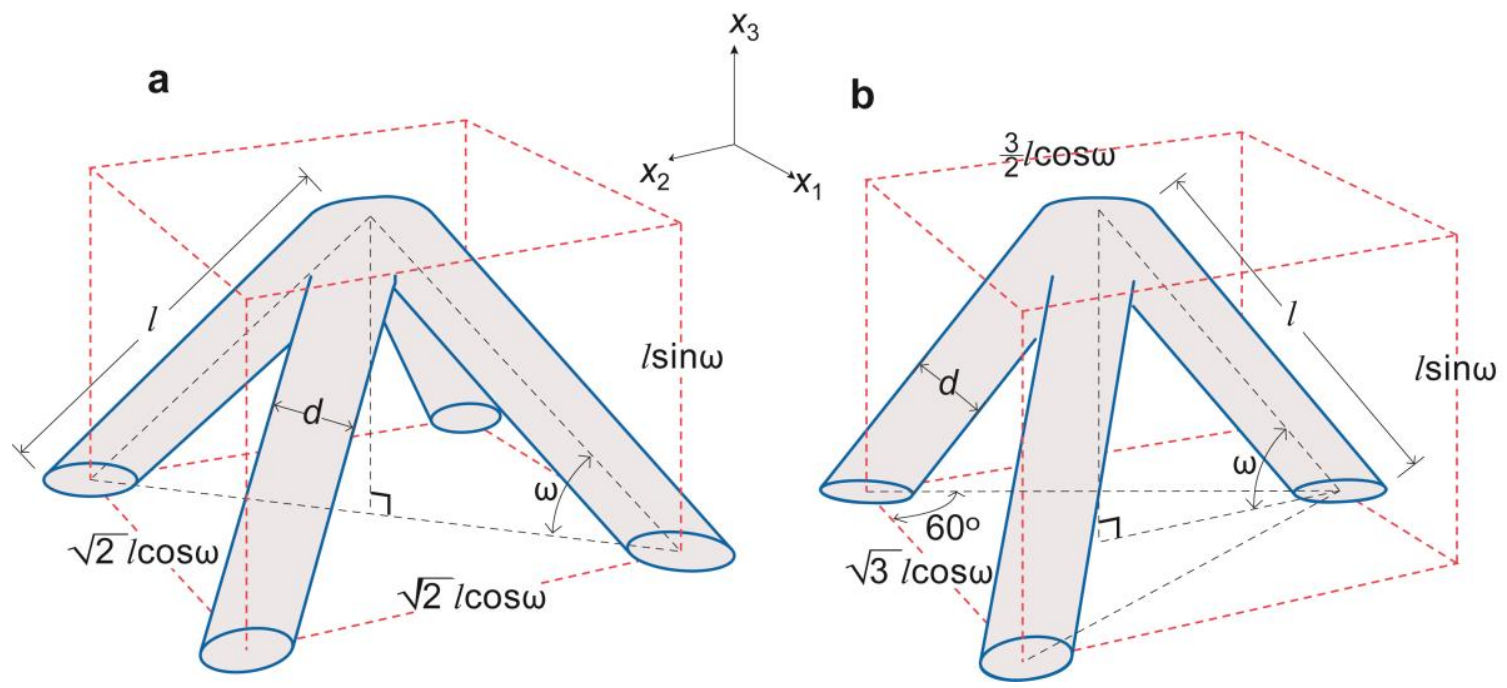

Figure 2.4 a) Pyramidal lattice unit cell with four struts, and b) tetrahedral lattice unit cell with three struts

The compressive modulus and compressive strength are the same for both the tetrahedral and pyramidal topologies for a given relative density and truss inclination angle, given that the geometric factors are the same for both lattices for through-thickness compression $^{25}$, except for elastic buckling at small strut aspect ratios. In both cases, the modulus and strength would be given by equations 2.14 and 2.15 respectively:

$$
E=E_{s} \bar{\rho} \sin ^{4} \omega
$$




$$
\sigma=\sigma_{Y} \bar{\rho} \sin ^{2} \omega
$$

where $E_{s}$ and $\sigma_{Y}$ are the elastic modulus and yield strength of the parent material of the trusses. The compressive strength is governed by elastic buckling for slender struts.

The shear strength of the core for both topologies is anisotropic, and depends on the angle $\varphi$ of the applied shear force ${ }^{25}$. The anisotropy is greater for the pyramidal core than the tetrahedral core, with a wider variation from the minimum to the maximum shear strength for the pyramidal core than the tetrahedral core ${ }^{25}$.

\subsection{Octet Lattice Structures}

Octet lattice structures are an attractive topology due to their near-isotropic properties, and the fact that the cell size is independent of face sheet separation. The basic structure of an octet lattice is shown in Figure 2.5. The structure in its simplest form, can be conceptually visualized as a multi-layered pyramidal lattice without face sheets, and with horizontal truss ligaments connecting the nodes of the structure. The relative density of the structure can be calculated the same way as other lattices, i.e by dividing the total truss volume by the volume of the unit cell. Considering a unit cell consisting of two inverted pyramidal units, along with the connected in-plane trusses, the total volume of 


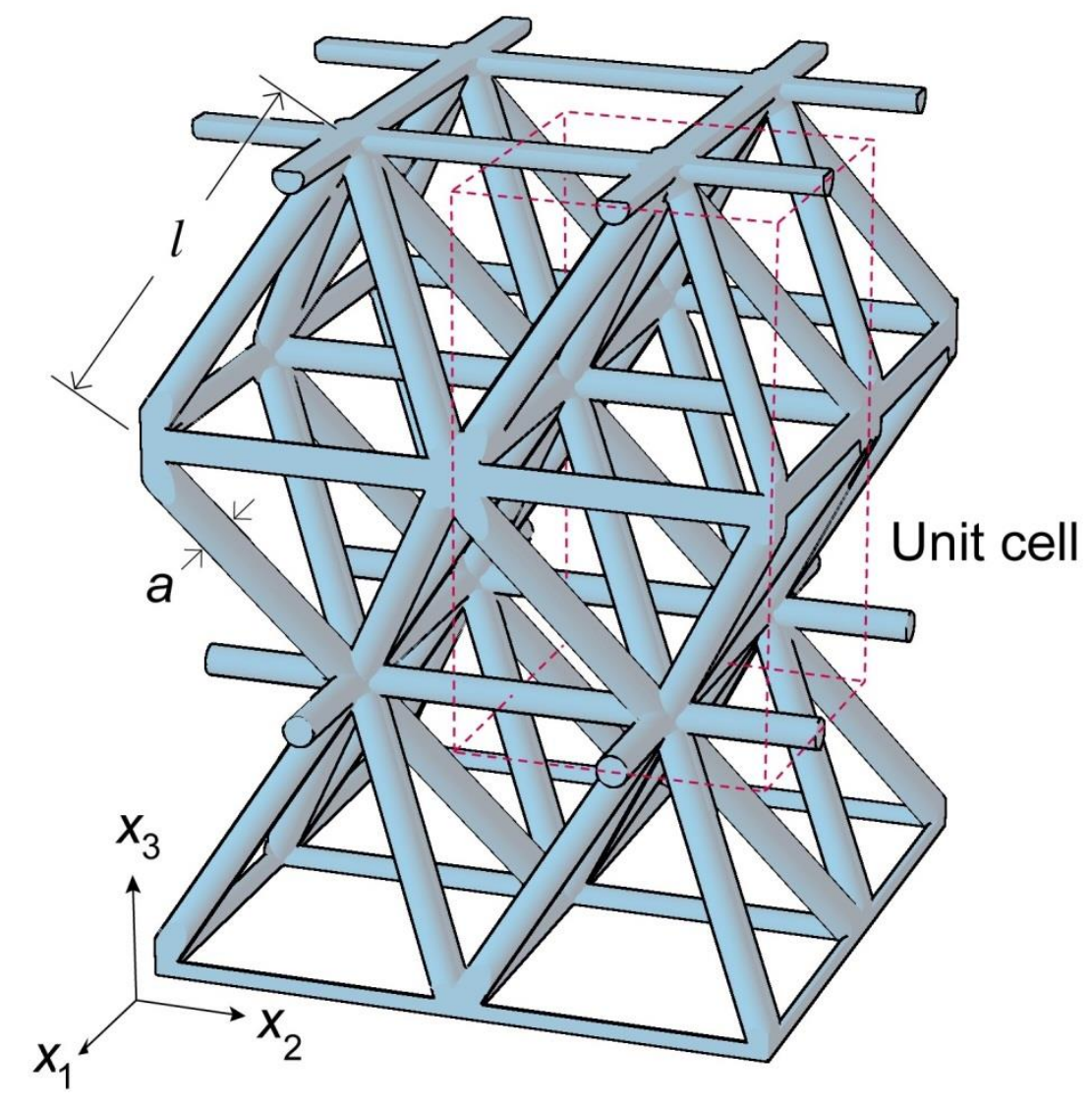

Figure 2.5: Octet lattice structure with circular cross-section trusses. The unit cell is shown in dashed lines.

truss can be calculated by counting the total number of trusses within the unit cell (12), and multiplying by the volume of the trusses $\left(\pi r^{2} l\right)$, where $r$ is the radius of the trusses $(a / 2)$. The volume of the unit cell is equivalent to $2 l^{3} \sin \omega$. For the regular octet lattice, the angle of inclination of the trusses $\omega$ is 45 degrees, thus, $\sin \omega$ is equivalent to $\sqrt{2} / 2$. Thus, the relative density of the octet lattice could be expressed as:

$$
\bar{\rho}=6 \pi \sqrt{2}\left(\frac{r}{l}\right)^{2}
$$


The above expression serves as a good first order approximation for the relative density for most cases, and does not account for the double counting of the node volume. For cases of a large r/l ratio, the double counting of the node volume becomes significant, and additional terms would have to be added to correct for this, based on the specific geometry of the nodes. The most comprehensive analysis of the elastic/plastic behavior of octet lattices was carried out by Deshpande, et $\mathrm{al}^{27}$, for metal octet lattices. The authors develop relations between the parent material properties and the elastic modulus and plastic collapse strength of the lattice for loading in a number of directions. This was done using stiffness matrices, backed by finite element simulations. The equations reviewed in this chapter offer an introduction to the concept of relating lattice mechanical behavior to parent material properties, relative density, and unit cell configuration. 


\section{Chapter 3}

\section{Laminated Carbon Fiber Structures}

As indicated in Chapter 2, the stiffness and strength of lattice structures depends upon the topology, relative density and the mechanical properties of the materials used to construct them $^{45}$. Sandwich structures with square honeycomb cores made from 0/90 crossply carbon fiber reinforced polymer (CFRP) composite laminates have recently been shown to offer a high specific strength in through-thickness compression ${ }^{26}$. CFRP sandwich panels with open cell pyramidal lattice cores have also been recently fabricated from $0 / 90$ crossply laminates using a snap fit process ${ }^{4}$. The specific compressive strength of this pyramidal lattice core was shown to be exceeded by only that of CFRP honeycombs for core densities around $100 \mathrm{kgm}^{-3}$. The high specific compressive strength of this lattice resulted from the resistance of trusses to elastic buckling and compressive delamination. However, sandwich panel structures are usually used in situations where they are subjected to significant bending loads ${ }^{1}$. In this case, the shear response of the core governs the panel's stiffness and strength. In this Chapter, the in-plane shear stiffness and strength of CFRP pyramidal lattice truss structures made by the snap fit assembly method ${ }^{4}$ is investigated, and its performance compared with other lightweight core concepts. 


\subsection{Materials and manufacture}

The pyramidal truss sandwich panels were manufactured from $0 / 90^{\circ}$ laminate sheets of thickness $t=3.175 \mathrm{~mm}$ in a three step process. First, patterns as shown in Figure 3.1a were water jet cut from the laminate sheets. Second, these patterns were then snap-fitted into each other (Figure 3.1b) to produce a pyramidal truss. Finally, the pyramidal truss was bonded to $3.175 \mathrm{~mm}$ thick composite face sheets using an epoxy adhesive. (The adhesive comprised 100 parts by weight of Dow Epoxy Resin with 13 parts of a triethylene tetramine curing agent. The cure cycle consisted of 12 hours at room temperature, followed by heating to $100^{\circ} \mathrm{C}$ for two hours.) These composite face sheets had cruciform shaped slots of depth $t_{0}$ milled into them at appropriate locations such that the pyramidal nodes of the pyramidal truss could be counter-sunk into the face sheets (Figure 3.1d).

The critical parameters describing the geometry of the pyramidal core are sketched in Figure 3.1c and include, the strut length $l$, the strut width $t$ (which is equal to the laminate sheet thickness and thus the struts have a square cross-section) and the node width and thickness $b$ and $t_{0}$, respectively. The struts make an angle $\omega$ with the horizontal plane of the face sheet (Figure 3.1c). The unit cell of the pyramidal core is sketched in Figure 3.2a. Simple geometric considerations dictate that the relative density of the core (defined as the density of the "smeared-out" core to the density of the solid material from which it is made) is given by:

$$
\bar{\rho}=\frac{2\left(l t+t_{0} b\right) t}{l \sin \omega(l \cos \omega+b)^{2}} \equiv \frac{2(\bar{l}+\overline{h b})}{\bar{l} \sin \omega(\bar{l} \cos \omega+\bar{b})^{2}},
$$


where the non-dimensional lengths $\bar{l} \equiv l / t, \bar{b} \equiv b / t$ and $\bar{h} \equiv t_{0} / t$. In the limit of vanishing node volumes $(b=h \rightarrow 0)$, this expression reduces to:

$$
\bar{\rho} \approx \frac{4}{\bar{l}^{2} \sin 2 \omega \cos \omega} .
$$

A photograph of the as-manufactured $\bar{\rho}=7 \%$ specimen is included in Figure 3.2c.

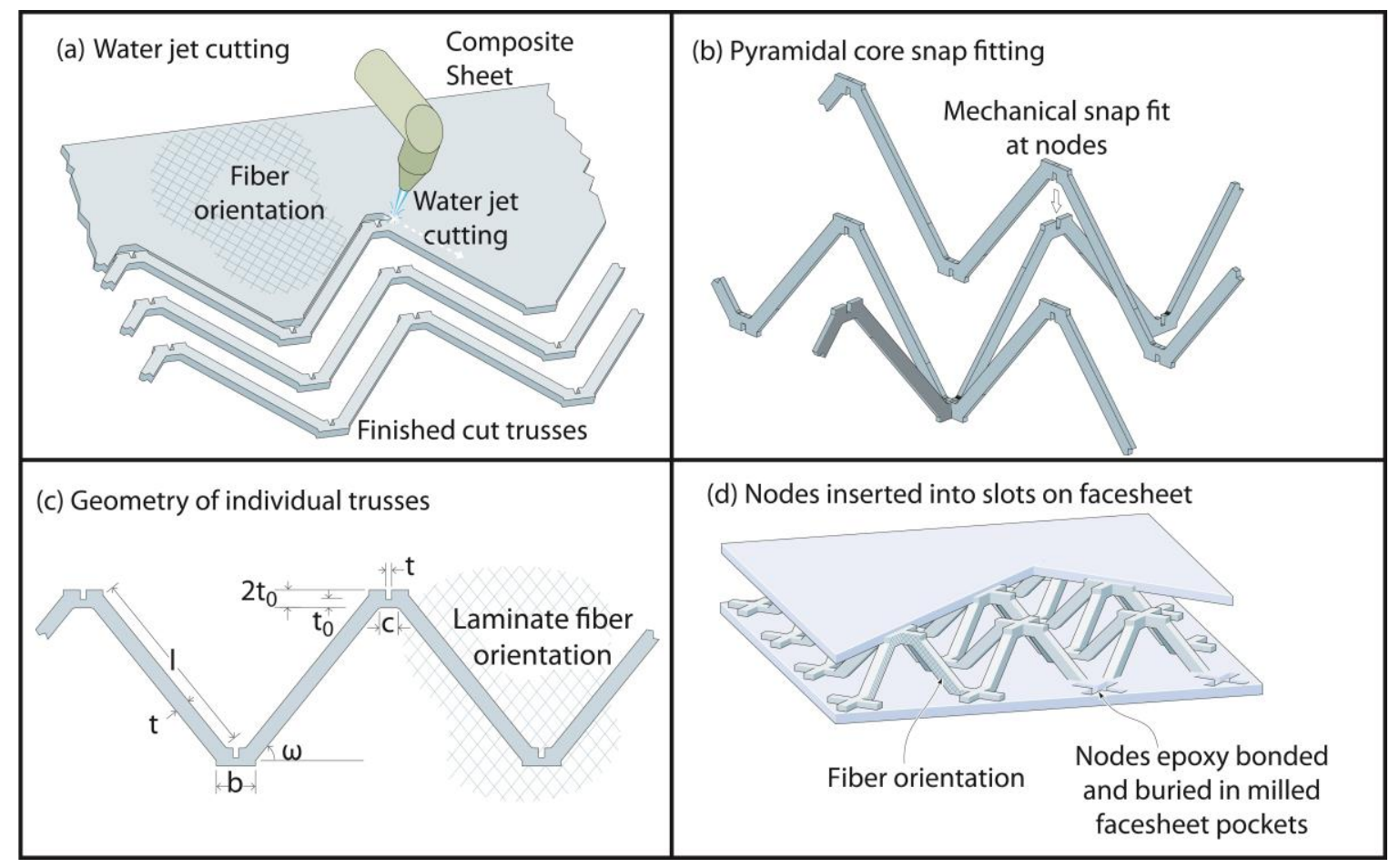

Figure 3.1: The fabrication route for making composite pyramidal lattice core sandwich panels. (a) Patterns were water jet cut from $0 / 90^{\circ} \mathrm{CFRP}$ laminate sheets. The fiber directions are shown in this sketch. (b) Snap-fitting of the patterns to produce a pyramidal lattice. (c) Geometry of the truss pattern with relevant core design variables defined. (d) Fully assembled pyramidal lattice core sandwich panel. The composite face-sheets utilized cruciform shaped slots into which the pyramidal trusses were fitted and adhesively bonded. 


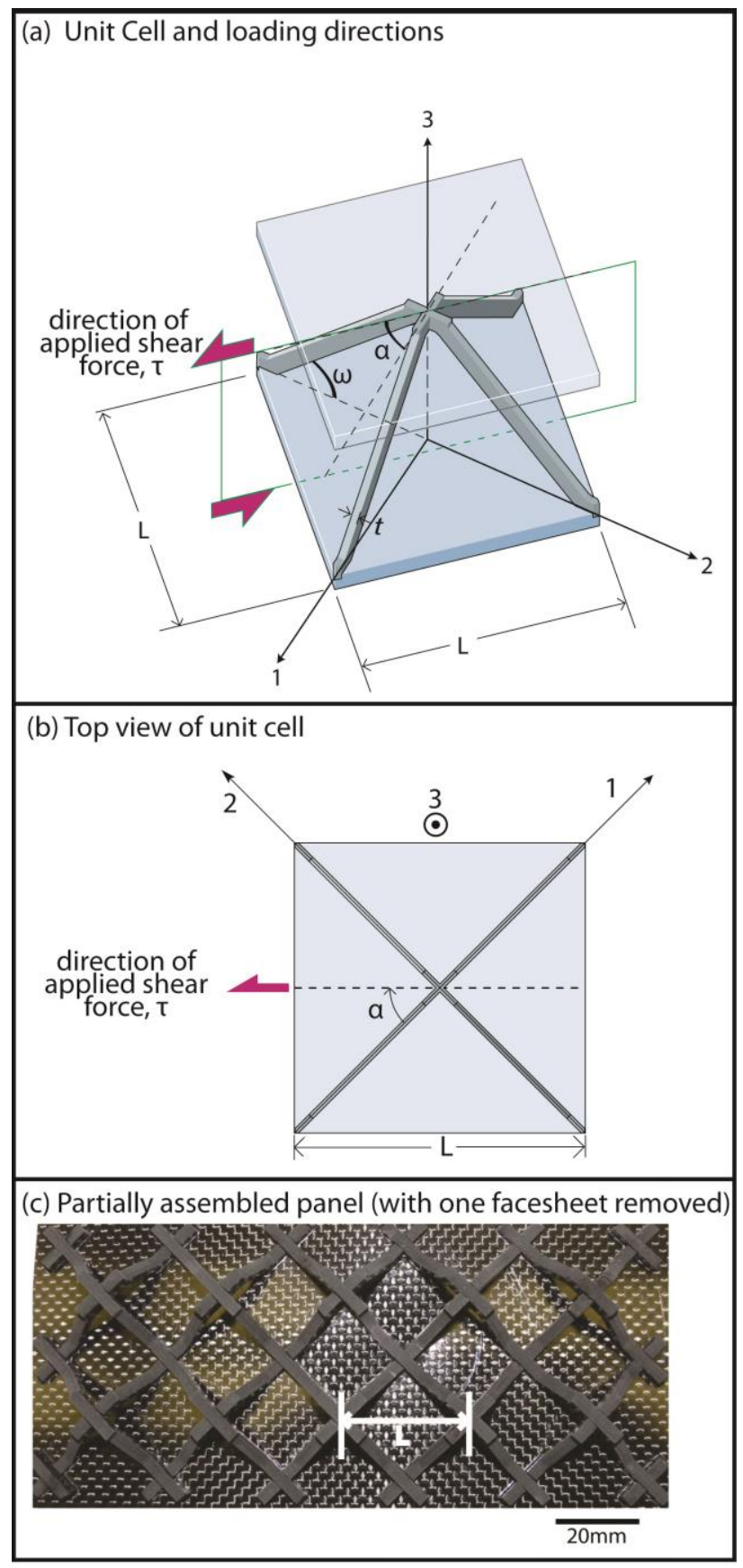

Figure 3.2: (a) Sketch of the unit cell of the pyramidal core with co-ordinate system marked. (b) Top-view of the unit cell indicating the direction of shear. (c) Photograph of the as-manufactured design 1 core $(\bar{\rho}=7 \%)$ in a sandwich panel structure. 


\subsubsection{Composite laminate material}

The 0/90 CFRP laminate sheets were obtained from McMaster-Carri. They had a thickness $t=3.175 \mathrm{~mm}$ and comprised $65 \%$ by volume of carbon fibers in a vinylester matrix. Plies comprising unidirectional fibers were laid-up alternating at $\left[0^{\circ} / 90^{\circ}\right]$ orientation to build an orthotropic laminate comprising 14 plies. The measured density of the laminate material was $\rho_{s}=1440 \mathrm{kgm}^{-3}$.

The laminate was tested in uniaxial compression along one of the fiber directions in order to determine the relevant Young's modulus and compressive strength of the parent material used to manufacture the pyramidal cores. Two types of compression tests were conducted:

(i) Column compression tests were conducted in which the specimens were compressed between two flat, parallel and rigid platens with no end-clamping of the laminates. They failed by interplay delamination, and this provided the delamination strength of the laminates and best simulated the loading conditions of the struts of the pyramidal core.

(ii) Combined loading compression (CLC) test in accordance with ASTM D $6641^{46}$ to determine the micro-buckling strength of the composite. This clamped test is selected to ensure that brooming failure does not occur within the specimen.

In each case tests were conducted on rectangular specimens of thickness $3.175 \mathrm{~mm}$, width $20 \mathrm{~mm}$ and gauge length $12 \mathrm{~mm}$ : the specimens were deliberately designed to be

\footnotetext{
${ }^{\mathrm{i}}$ McMaster-Carr, 6100 Fulton Industrial Blvd. Atlanta, GA 30336-2852, USA.
} 
sufficiently "stocky" so as to prevent the Euler buckling of the specimens. The applied load was measured via a load cell while a laser extensometer was used to measure the nominal axial strain in the specimens. A nominal applied strain rate of $10^{-3} \mathrm{~s}^{-1}$ was employed for these tests. The measured compressive nominal stress versus strain curves from both types of tests are plotted in Figure 3.3. The following can be deduced: (i) the unloading Young's modulus $E_{s} \approx 28 \mathrm{GPa}$, (ii) the delamination failure strength $\sigma_{d l} \approx 380 \mathrm{MPa}$ while (iii) the micro-buckling strength of the composite is $\sigma_{\max }=615 \mathrm{MPa}$. The tensile strength of the laminate was measured in accordance to ASTM D3039 standard for composite testing, using 20mm wide coupons and a 50mm gage length. Five specimens were tested for each for each condition, and the average laminate properties are listed in Table 3.1.

Table 3.1: [0/90] CFRP Laminate Properties

\begin{tabular}{|c|c|c|c|c|c|}
\hline Density & $\begin{array}{c}\text { Young's } \\
\text { Modulus }\end{array}$ & $\begin{array}{c}\text { Microbuckling } \\
\text { Strength }\end{array}$ & $\begin{array}{c}\text { Delamination } \\
\text { Strength }\end{array}$ & $\begin{array}{c}\text { Tensile } \\
\text { Strength }\end{array}$ & $\begin{array}{c}\text { Tensile } \\
\text { Modulus }\end{array}$ \\
\hline$\rho\left(\mathrm{Mg} / \mathrm{m}^{3}\right)$ & $\mathrm{E}(\mathrm{GPa})$ & $\sigma_{m b}(\mathrm{MPa})$ & $\sigma_{d l}(\mathrm{MPa})$ & $\sigma_{t}(\mathrm{MPa})$ & $\mathrm{Y}(\mathrm{GPa})$ \\
\hline 1.4 & 28 & 615 & 380 & 450 & 77 \\
\hline
\end{tabular}



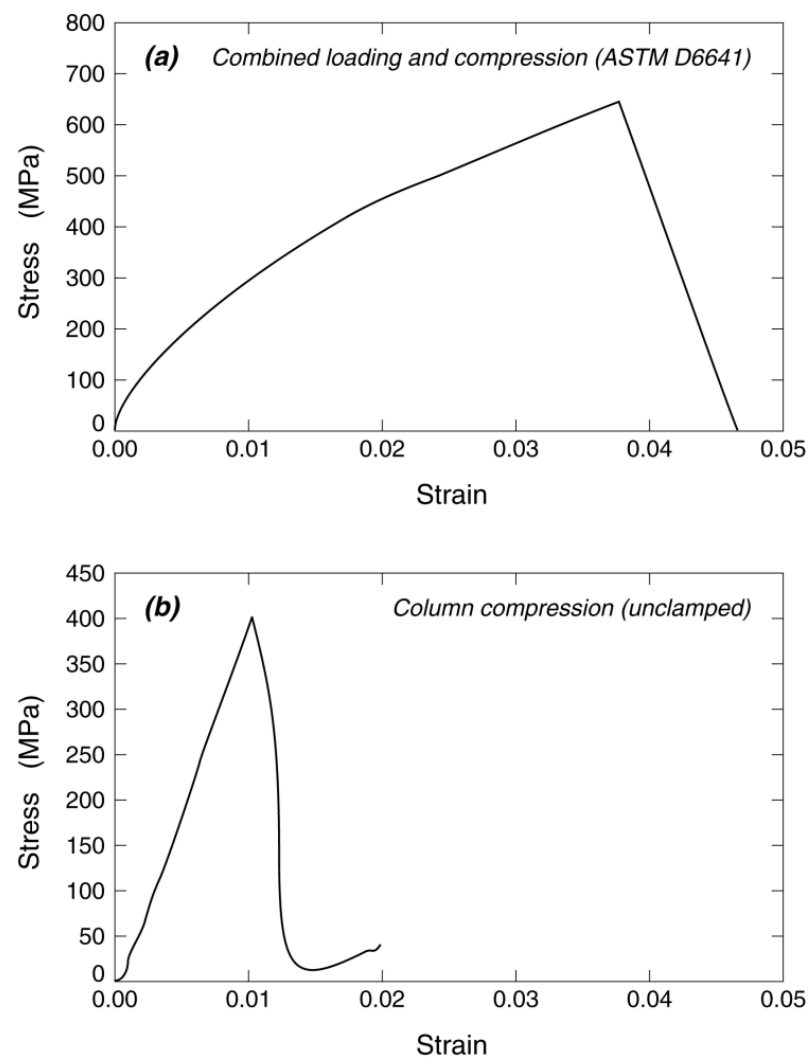

Figure 3.3: The measured uniaxial compressive stress versus strain response of the composite material along one of the fiber directions. Results for both a CLC test (a) and a column compression test (b) are shown.

\subsubsection{Pyramidal core designs}

All the pyramidal cores tested and manufactured in this study had a strut angle $\omega=45^{\circ}$. Therefore, the included angle between the struts was $90^{\circ}$ and the patterns were cut from the laminate sheets such that half the fibers of the $0-90^{\circ}$ laminate were aligned along the axis of the struts of the pyramidal core (Figure 3.1a). Panels with a pyramidal core of relative density $\bar{\rho}$ ranging between $0.9 \%$ to $10 \%$ were manufactured and tested 
in this study. All the cores had a strut thickness $t=3 \mathrm{~mm}$ and node width $b=15.3 \mathrm{~mm}$ and the strut length $l$ was varied between $9.4 \mathrm{~mm}$ and $63.5 \mathrm{~mm}$ to enable the manufacturing of cores of different densities. The main part of the study was carried out on cores with node thickness $t_{0}=0.8 t$ : a parametric study (reported subsequently in Chapter 3.2) confirmed that node failure was not an operative failure mechanism during shear loading of the core for nodes with $t_{0} \geq 0.8 t$. The strut lengths $l$ of the six core relative densities investigated in this study are listed in Table 3.2.

Table 3.2: Sandwich Core Parameters

\begin{tabular}{|c|c|c|}
\hline $\begin{array}{c}\text { Density }(\bar{\rho}) \\
(\%)\end{array}$ & $\begin{array}{c}\text { Core height } h \\
(\mathrm{~mm})\end{array}$ & $\begin{array}{c}\text { Truss length, } l \\
(\mathrm{~mm})\end{array}$ \\
\hline 0.9 & 45.2 & 63.5 \\
\hline 1.6 & 31.4 & 44.1 \\
\hline 3.0 & 20.0 & 28.3 \\
\hline 5.0 & 13.2 & 18.7 \\
\hline 7.0 & 9.7 & 13.7 \\
\hline 10.0 & 6.6 & 9.4 \\
\hline
\end{tabular}

\subsection{Measurements of the shear response of the pyramidal}

\section{core}

Single lap shear tests were conducted on the pyramidal core panels in accordance with ASTM C- $273^{47}$, the standard test for sandwich core materials, using a compression plate setup as sketched in Figure 3.4. The ASTM standard specifies that the length to thickness ratio of the specimen $L / H \geq 12$, while the width $W \geq 2 H$, where $\mathrm{L}, \mathrm{W}$, and $\mathrm{H}$ are the length, width, and height of the lattice respectively. This was achieved by employing specimens that had 2 pyramidal cells along the width of the specimen and 5 
to 7 cells along the length depending upon the specimen relative density ( 5 cells were used for specimens with $5 \% \leq \bar{\rho} \leq 10 \%, 6$ cells for the $3 \%$ and $1.6 \%$ specimens while the $0.9 \%$ specimen required 7 cells). In order to attach the specimen to the test fixture, holes were drilled into the composite face sheets of the pyramidal core panels and the panels attached to the text fixture (Figure 3.4). The applied load was measured from the load cell of the test machine and used to infer the shear stress while the shear strain was measured using a laser extensometer. All tests were conducted at an applied shear strain rate of $10^{-3} \mathrm{~s}^{-1}$

In general, the shear response of the pyramidal core is anisotropic and hence dependent on the direction of shearing. The direction of shearing was defined as follows. Consider the unit cell sketched in Figure 3.2a: the shearing direction is specified via the angle $\alpha$ (Figure 3.2b) such that $\alpha=0^{\circ}$ and $90^{\circ}$ for applied shear stresses $\tau_{13}$ and $\tau_{23}$, respectively. Unless otherwise specified, all measurements reported subsequently are for $\alpha=45^{\circ}$ and for the sake of brevity we shall refer to this shear stress via the symbol $\tau$ and the corresponding engineering shear strain via $\gamma$. 


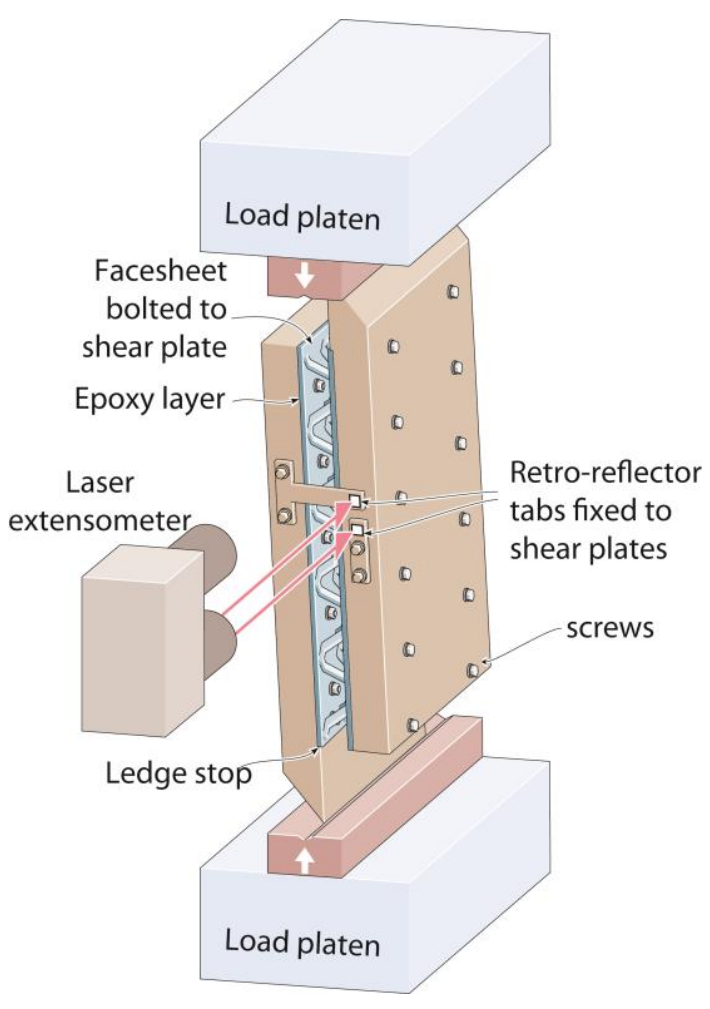

Figure 3.4: Sketch of the single lap shear compression plate setup used to measure the shear response of the pyramidal cores.

\subsubsection{Effect of node design}

The measured $\tau$ versus $\gamma$ curves for the $\bar{\rho}=5 \%$ pyramidal core are plotted in Figure 3.5a for node thicknesses over the range $0.5 \leq t_{0} / t \leq 0.9$ (One specimen is tested for each node condition). The peak shear strength is seen to increase with increasing $t_{0} / t$ for $t_{0} / t<0.8$ with the shear response of the $t_{0} / t=0.8$ and 0.9 specimens identical for all practical purposes. The lower strength of the specimens with the smaller node thicknesses is due to tensile failure of the struts at the nodes as seen from the photograph in Figure 3.6a. A sketch illustrating this failure mode is shown in Figures 3.6b-d: the 
strut making an obtuse angle with the loading direction is under tensile stresses and breaks off at the nodes. This failure mode is prevented by employing sufficiently stubby nodes whereby the failure mode switches to failure of the struts of the pyramidal core and independent of the node thickness (see Figure 3.5b). Subsequently, all results are shown for $t_{0} / t=0.8$ such that node failure is never an operative mechanism.
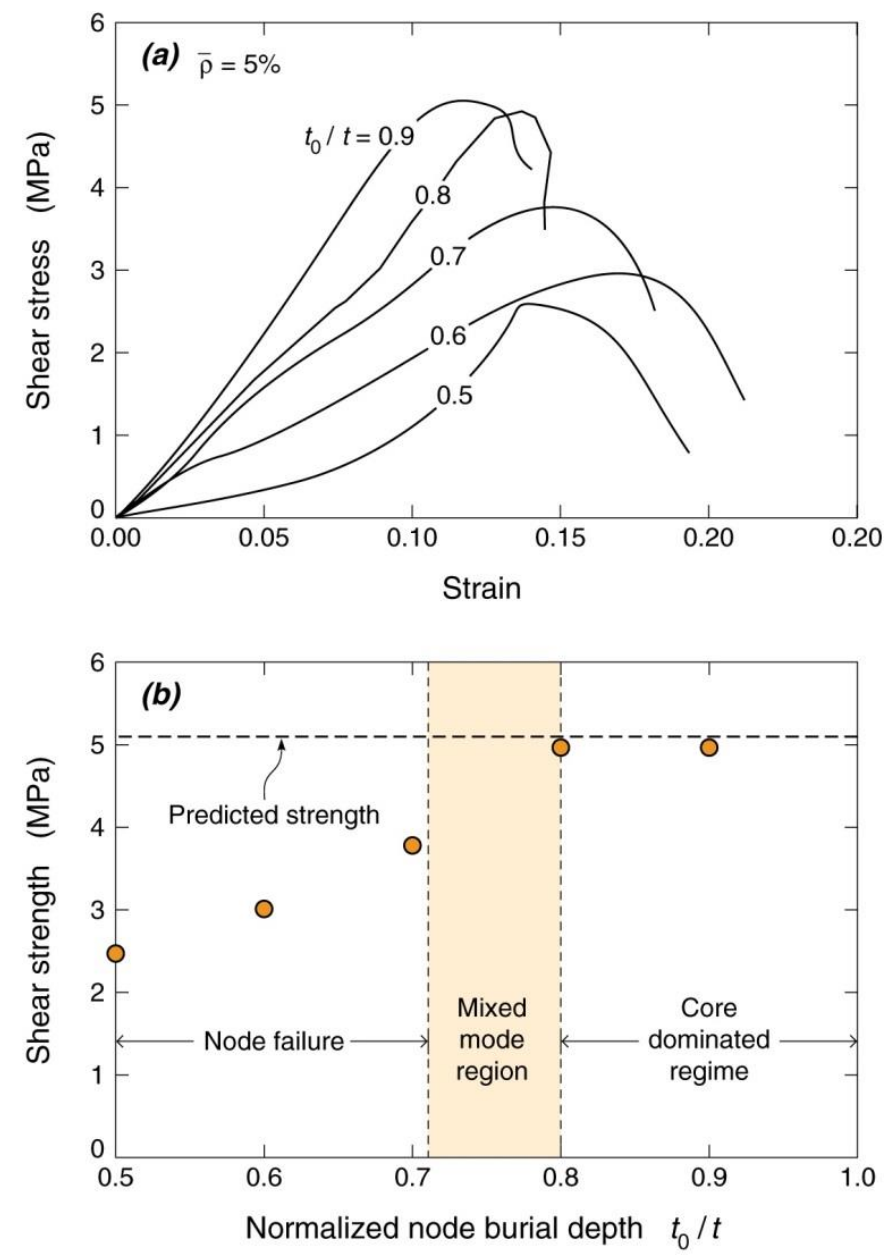

Figure 3.5: (a) The measured shear stress $\tau$ versus strain $\gamma$ response $\left(\alpha=45^{\circ}\right)$ of the $\bar{\rho}=5 \%$ core for selected values of the node thickness $t_{0}$. (b) The measured peak strength as a function of node thickness for the $\bar{\rho}=5 \%$ core. The predicted core strength (see Chapter 4) is also shown. 


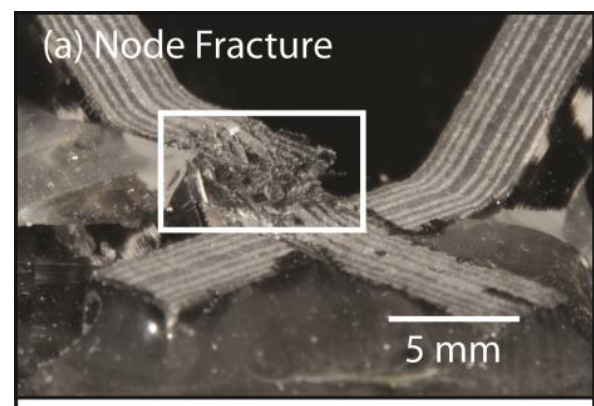

(b) Node design

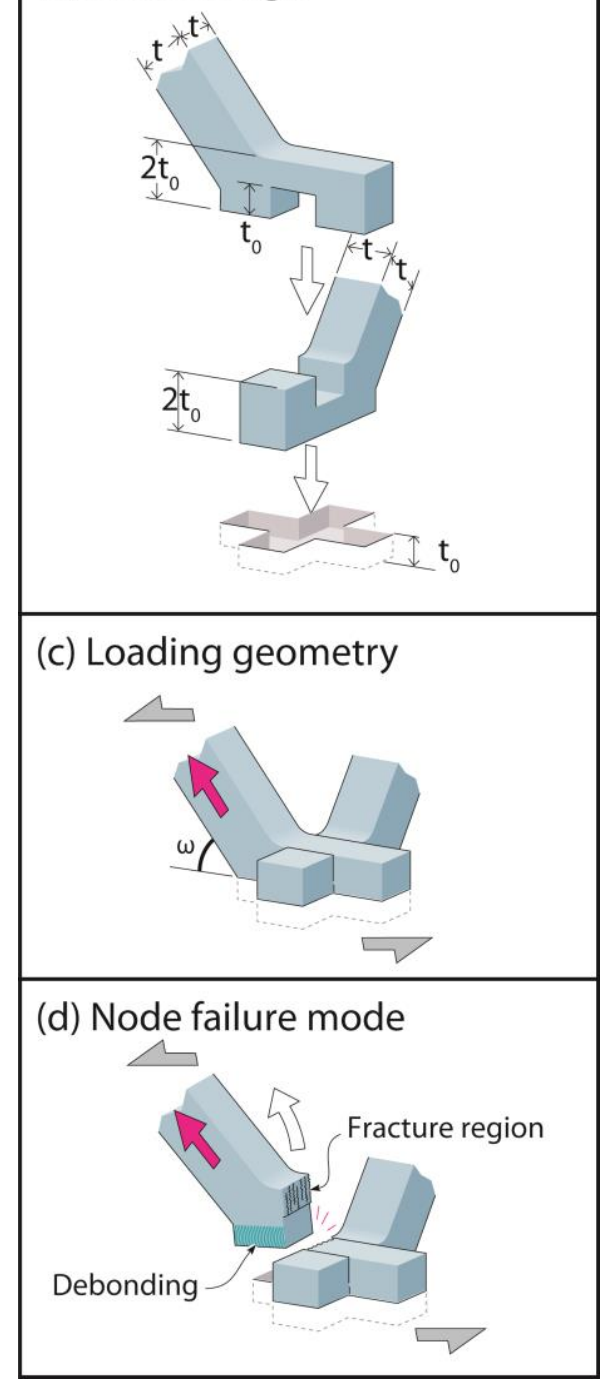

Figure 3.6: (a) Photograph of the node failure mechanism in the $\bar{\rho}=5 \%$ core with $t_{0} / t=0.5$. (b) Sketch illustrating the node failure mechanism. 


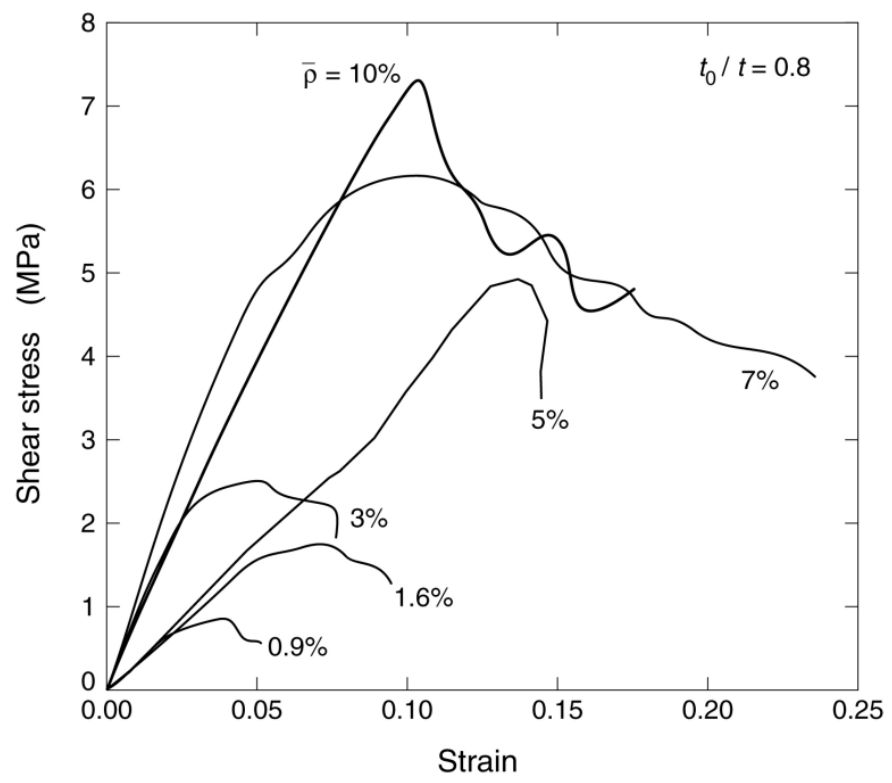

Figure 3.7: The measured shear stress $\tau$ versus strain $\gamma$ response $\left(\alpha=45^{\circ}\right)$ of the pyramidal cores for selected values of the relative density $\bar{\rho}$ and node thickness $t_{0} / t=0.8$.

\subsubsection{Effect of core relative density $\bar{\rho}$}

The measured shear stress $\tau$ versus strain $\gamma$ response of the pyramidal cores is plotted in Figure 3.7 for the six relative densities investigated here. Three samples were tested for each core relative density. In all cases, the responses display an initial linear elastic phase followed by a non-elastic phase, which typically precedes the peak stress and failure, and signals crack initiation and growth within the specimen. Failure first occurs in the strut under compression (i.e. the strut making an acute angle with the 
loading direction) and is followed by failure of the remaining struts and a consequent fall in the load carrying capacity of the specimen. Two failure modes were observed:

(i) Delamination failure of the struts: for all specimens expect for the $\bar{\rho}=0.9 \%$ specimens, the first failure event was delamination of the strut making an acute angle with the loading direction and thus under compression. A photograph of this failure event in the $\bar{\rho}=5 \%$ specimen just after the peak stress had been attained is included in Figure 3.8a. Subsequently, the other struts also fail by a combination of delamination and tensile fracture.

(ii) Euler buckling of the struts: the first failure event in the $\bar{\rho}=0.9 \%$ specimens was elastic Euler buckling of the strut under compressive loading (i.e. the strut making an acute angle with the loading direction). This failure event is shown in the photograph in Figure 3.8b taken immediately after the peak stress had been attained. The buckling event results in delamination of the strut followed by failure of the remaining three struts as well.

The measured unloading modulus and peak strengths are summarized in the next chapter, respectively as a function of the relative density $\bar{\rho}$.

\subsubsection{Effect of shearing direction $\alpha$}

All the results presented above were for loading in the $\alpha=45^{\circ}$ direction. To investigate the anisotropy, two additional tests were conducted on the $\bar{\rho}=5 \%$ core for loading in the $\alpha=22.5^{\circ}$ and $0^{\circ}$ directions. A comparison between the measured shear stress versus shear strain responses for the three loading directions is shown in Figure 
3.9: while the shear modulus seems to be relatively insensitive to $\alpha$, the peak shear strength decreases with decreasing $\alpha$ from approximately $4.5 \mathrm{MPa}$ in the $\alpha=45^{\circ}$ case to 2.7 MPa in the $\alpha=0^{\circ}$ case. The next chapter (Chapter 4) utilizes micromechanical models to understand the shear behavior of the lattice.

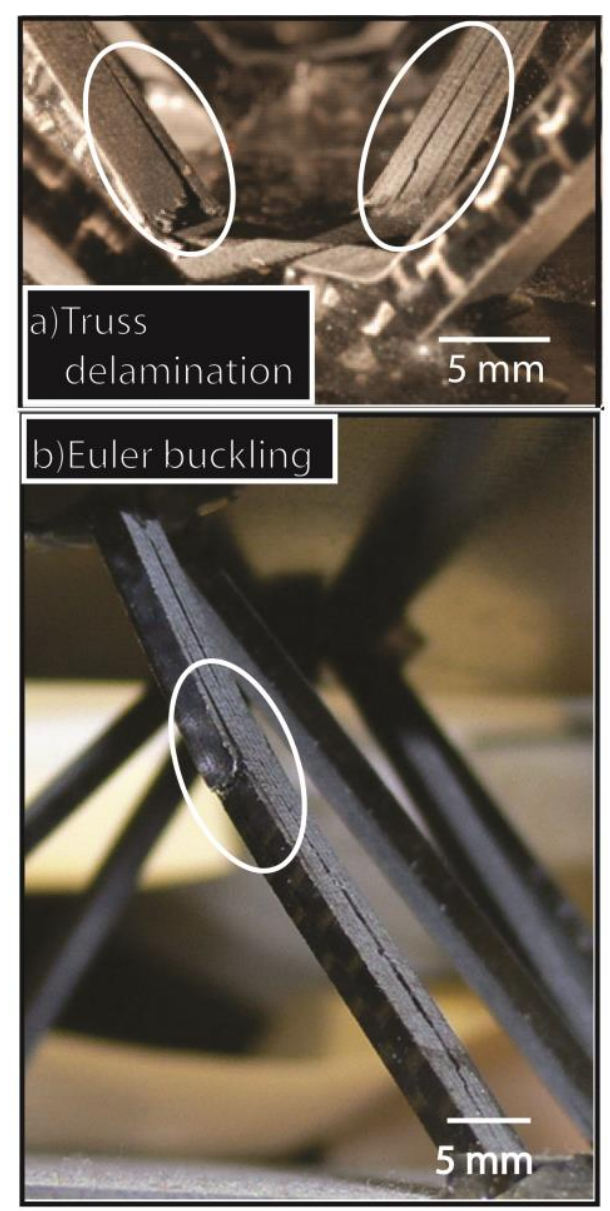

Figure 3.8: Photographs of specimens just after attainment of the peak load to illustrate the observed failure mechanisms. (b) Delamination observed in the $\bar{\rho}=5 \%$ specimen and (b) Euler buckling of the struts observed in the $\bar{\rho}=0.9 \%$ specimen. 


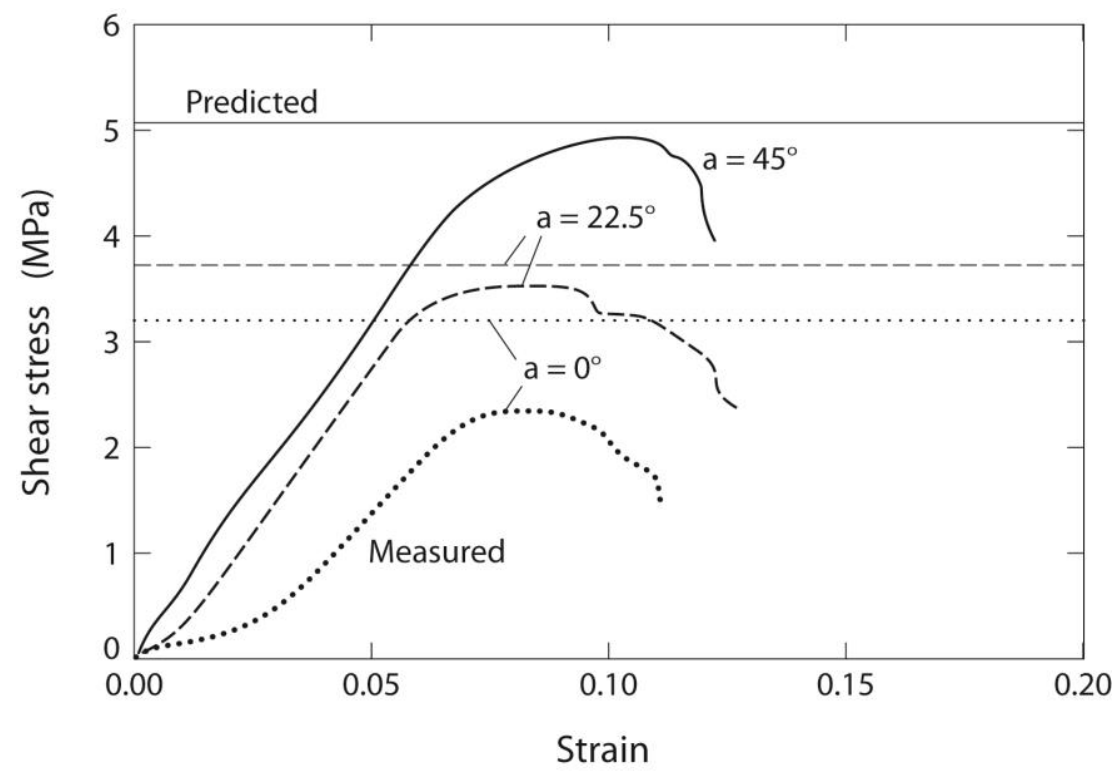

Figure 3.9: The measured shear stress $\tau$ versus strain $\gamma$ response of the $\bar{\rho}=5 \%$ pyramidal (node thickness $t_{0} / t=0.8$ ) for three selected loading directions $\alpha$. The analytical model predictions of the strength are also included. 


\section{Chapter 4}

\section{Analytical Model of the Shear Response}

In this chapter, the analytical expressions for the "effective" shear stiffness and strength of the composite pyramidal cores, sandwiched between two rigid face-sheets are derived. The pyramidal trusses are made from 0/90 CFRP laminates such that one set of fibers are aligned with the axial direction of the struts of the pyramidal truss (Figure 4.1a). A local Cartesian co-ordinate system $\left(e_{1}-e_{2}\right)$ is defined, aligned with the orthogonal set of fibers (Figure 4.1b). The Young's modulus and compressive plastic micro-buckling strengths of the laminate in either the $e_{1}$ or $e_{2}$ directions are $E_{s}$ and $\sigma_{c}$, respectively while $\tau_{Y}$ is the longitudinal shear strength of the matrix material of the laminate. The delamination strength of the composites along the $e_{1}$ or $e_{2}$ directions is denoted by $\sigma_{d l}$.
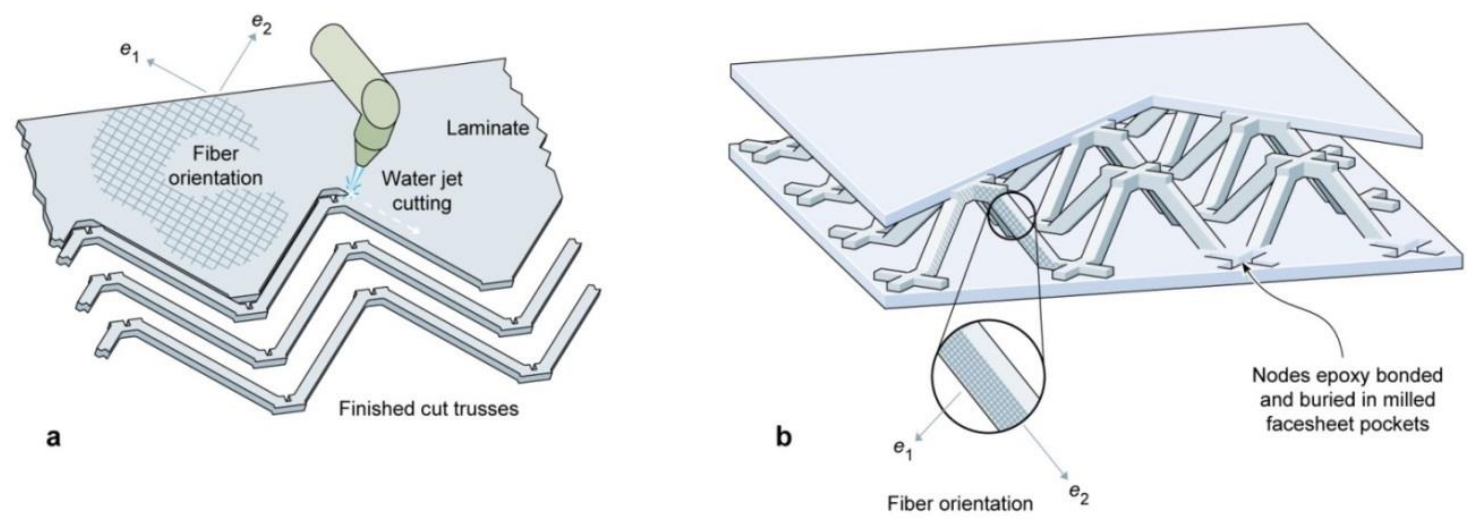

Figure 4.1: Schematics showing the fiber alignment on (a) the laminate sheets, and (b) The sandwich panel. 


\subsection{Elastic properties}

Analytical expressions for the shear modulus $G$ of the pyramidal core is obtained in terms of the core geometry and the elastic properties of the solid material by first analyzing the elastic deformations of a single strut of the pyramidal core and then extending the results to evaluate the effective properties of the core.

Consider the unit cell sketched in Figure 4.2a with an applied shear displacement $\delta$ at an angle $\alpha$ with the $x_{1}$ axis as shown in Figure 4.2b. We resolve this applied displacement into two perpendicular components:

$$
\delta_{1}=\delta \cos \alpha
$$

and

$$
\delta_{2}=\delta \sin \alpha .
$$

The struts in the unit cell are labeled via the symbols A through D as shown in Figure 4.2a in order to refer to them in the remainder of the analysis. The axial and shear displacements applied to struts $\mathrm{A}$ and $\mathrm{C}$ are:

$$
\delta_{a}=\delta_{1} \cos \omega,
$$

and

$$
\delta_{s}=\delta_{1} \sin \omega
$$

respectively with strut $\mathrm{A}$ under a compression while strut $\mathrm{C}$ is subjected to a tensile displacement. Elementary beam theory gives the axial and shear forces in strut A (or C) as:

$$
F_{A}=E_{s} t^{2} \frac{\delta_{a}}{l},
$$

and 


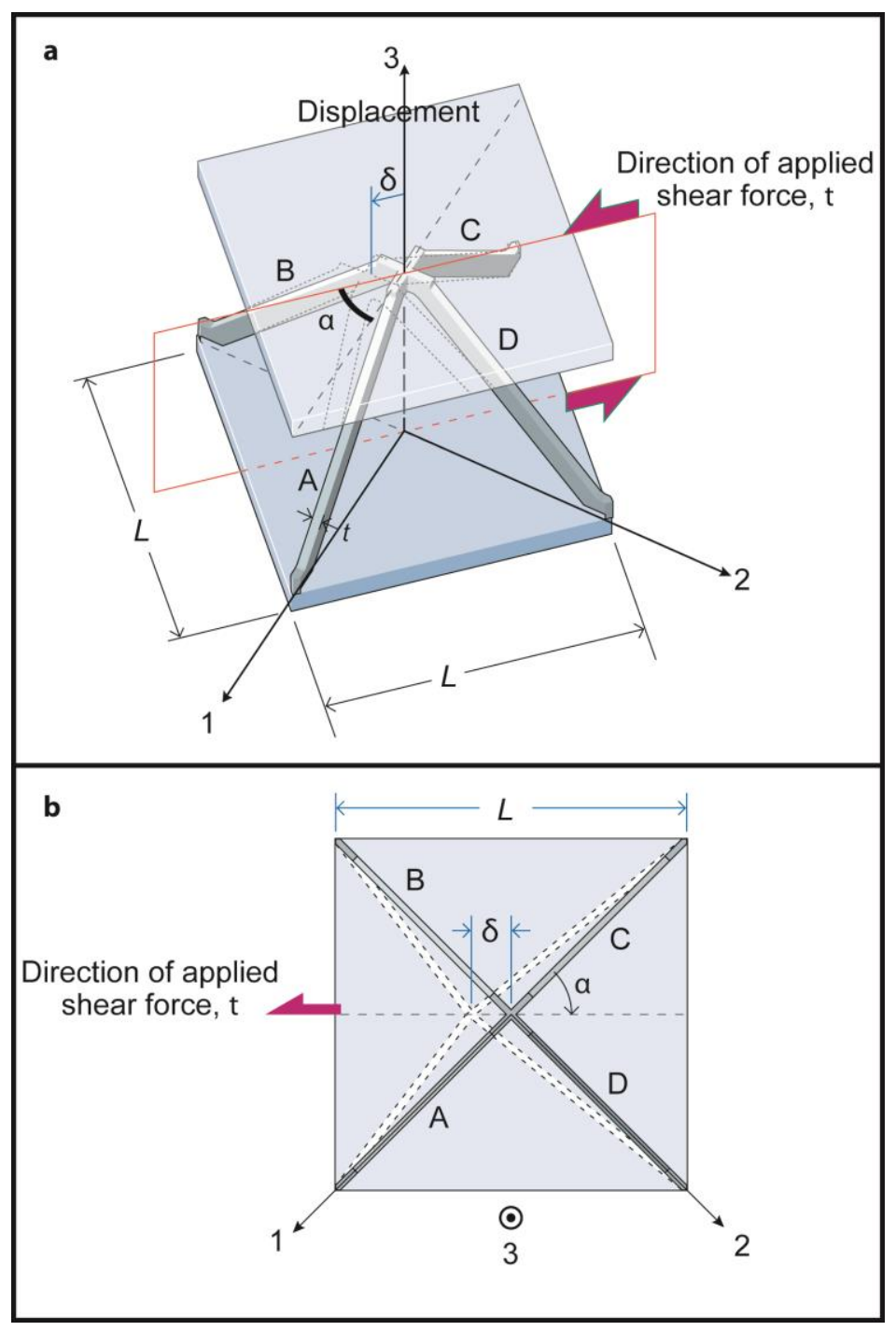

Figure 4.2 (a) Oblique and (b) planer views of the unit showing truss displacement due to an applied shear force.

$$
F_{S}=\frac{12 E_{s} I \delta_{s}}{l^{3}}
$$

respectively, where $I \equiv t^{4} / 12$ is the second moment of area of the strut cross-section.

Equivalent expressions are valid for struts B and D by replacing $\delta_{1}$ with $\delta_{2}$ in Equation (4.2). The total applied force along the $x_{1}$ direction then follow as 


$$
F_{1}=2\left(F_{A} \cos \omega+F_{S} \sin \omega\right)=\frac{2 E_{s} t^{2} \delta_{1}}{l}\left[\cos ^{2} \omega+\left(\frac{t}{l}\right)^{2} \sin ^{2} \omega\right]
$$

while the force $F_{2}$ in the $x_{2}$ direction is obtained by replacing $\delta_{1}$ with $\delta_{2}$ in Equation

(4.4). The applied shear stress is then given by:

$$
\tau \equiv \frac{2 \sqrt{F_{1}^{2}+F_{2}^{2}}}{(2 l \cos \omega+2 b)^{2}}
$$

and the engineering shear strain is:

$$
\gamma \equiv \frac{\delta}{l \sin \omega}
$$

The effective shear modulus $G \equiv \tau / \gamma$ of the pyramidal core then follows from Equations (4.5) and (4.6) as:

$$
\frac{G}{E_{s}}=\frac{\sin \omega}{(\bar{l} \cos \omega+\bar{b})^{2}}\left[\cos ^{2} \omega+\frac{\sin ^{2} \omega}{\bar{l}^{2}}\right]
$$

in terms of the non-dimensional geometric parameters of the core $\bar{l} \equiv l / t$ and $\bar{b} \equiv b / t$.

In the limit of negligible node volumes (i.e. $\bar{b}=\bar{h} \rightarrow 0$ ), the dimensionless modulus $G / E_{s}$ is related to the relative density $\bar{\rho}$ of the core via:

$$
\frac{G}{E_{s}} \approx \frac{\sin \omega}{\bar{l}^{2} \cos ^{2} \omega}\left[\cos ^{2} \omega+\frac{\sin ^{2} \omega}{\bar{l}^{2}}\right]=\frac{\bar{\rho}}{8} \sin ^{2} 2 \omega+\frac{\bar{\rho}^{2}}{16} \sin ^{4} \omega \cos ^{2} \omega
$$

Equation 4.8 has been written so that the first and second terms represent the contributions to the stiffness of the pyramidal core due to the stretching and bending of the struts respectively. 


\subsection{Collapse strength}

Given a robust node-face sheet connection, and that the laminate tensile strength exceeds the delamination strength, the struts under compression determine the strength of the lattice. There are three critical collapse mechanisms for the pyramidal core struts: (i) plastic micro-buckling; (ii) delamination failure and (iii) elastic Euler bucking. The operative failure mode will be the one associated with the lowest value of the collapse strength. Typically polymer matrices of fiber composites display non-linear behavior ${ }^{48}$ and thus elastic micro-buckling is not an operative failure mode and not considered in the collapse calculations presented here.

Before considering each of these failure modes, in turn, we derive expressions relating the failure strength of the pyramidal core to the compressive failure strength $\sigma_{c}$ of a single strut. The ratio of the shear to axial forces in the struts is given from Equations (4.2) and (4.3) as:

$$
\frac{F_{S}}{F_{A}}=\left(\frac{t}{l}\right)^{2} \tan \omega
$$

Substituting Equation (4.9) in Equations (4.4) and (4.5), the peak shear stress $\tau_{p}$ is given in terms of $\sigma_{c}$ as:

$$
\frac{\tau_{p}}{\sigma_{c}}=\frac{\left[\cos \omega+\left(\frac{t}{l}\right)^{2} \frac{\sin ^{2} \omega}{\cos \omega}\right]}{\cos \alpha(\bar{l} \cos \omega+\bar{b})^{2}}
$$

for $\alpha \leq \pi / 4$ : the peak strength in this case is set by the compressive failure of strut $\mathrm{A}$ (Figure 4.2) at a stress $\sigma_{c}$, because that strut experiences a higher resolved component of 
the shear force. Similarly, for $\pi / 4<\alpha \leq \pi / 2$, compressive failure first occurs in strut B and the failure stress follows as:

$$
\frac{\tau_{p}}{\sigma_{c}}=\frac{\left[\cos \omega+\left(\frac{t}{l}\right)^{2} \frac{\sin ^{2} \omega}{\cos \omega}\right]}{\sin \alpha(\bar{l} \cos \omega+\bar{b})^{2}}
$$

We now proceed to derive expressions for $\sigma_{c}$ for each of the failure modes considered here.

\subsubsection{Plastic micro-buckling of the composite struts}

It is generally accepted that fiber micro-buckling of composites is an imperfection-sensitive, plastic buckling event involving the non-linear longitudinal shear of the composite within a narrow kink band. Argon ${ }^{49}$ argued that the compressive strength $\sigma_{\max }$ is dependent on the shear strength of the surrounding matrix, $\tau_{Y}$, and the misalignment angle of the fibers $\phi$, and is given by:

$$
\sigma_{\max }=\frac{\tau_{Y}}{\bar{\phi}}
$$

for a composite comprising inextensional fibers and a rigid-ideally plastic matrix of shear strength $\tau_{Y}$. Kinking initiates from a local region of fiber misalignment of angle $\bar{\phi}$. It is assumed that the micro-buckle band is transverse to the axial fiber direction $e_{1}$, such that the angle $\beta$ between the normal to the band and the fiber direction vanishes. Now consider the case where the remote stress state consists of an in-plane shear stress $\tau^{\infty}$ in addition to a compressive stress parallel to the fibers. Then, Fleck and Budiansky ${ }^{50}$ have 
shown that this in-plane shear stress leads to a reduction in the micro-buckling stress as shown below:

$$
\sigma_{c}=\frac{\tau_{Y}-\tau^{\infty}}{\bar{\phi}}
$$

Prior to the micro-buckling of the struts, the struts are elastic and the analysis detailed above applies. Thus, from Equations (4.9) and (4.12) it follows that the axial stress $\sigma_{c}$ required to initiate micro-buckling in the inclined strut is given by:

$$
\sigma_{c}=\frac{\tau_{Y}}{\left[\bar{\phi}+\tan \omega\left(\frac{t}{l}\right)^{2}\right]}=\frac{\sigma_{\max }}{\left[1+\frac{\tan \omega}{\bar{\phi}}\left(\frac{t}{l}\right)^{2}\right]},
$$

where $\sigma_{\max }$ is the micro-buckling strength of the laminate for loading in the $e_{1}$-direction in the absence of remote shear. Substituting Equation (4.13) in Equation (4.10a) gives the shear strength as:

$$
\frac{\tau_{p}}{\sigma_{\max }}=\frac{\left[\cos \omega+\left(\frac{t}{l}\right)^{2} \frac{\sin ^{2} \omega}{\cos \omega}\right]}{\cos \alpha\left[1+\frac{\tan \omega}{\bar{l}^{2} \bar{\phi}}\right](\bar{l} \cos \omega+\bar{b})^{2}}
$$

for $\alpha \leq \pi / 4$. In the limit of vanishing node volume, the above expression reduces to:

$$
\frac{\tau_{p}}{\sigma_{\max }}=\frac{\bar{\rho} \sin 2 \omega}{4\left[1+\frac{\bar{\rho} \sin \omega \sin 2 \omega}{4 \bar{\phi}}\right]} \frac{\left[1+\frac{\bar{\rho} \sin ^{3} \omega}{2}\right]}{\cos \alpha}
$$

The shear strength for the case of $\pi / 4<\alpha \leq \pi / 2$ is given by replacing $\cos \alpha$ by $\sin \alpha$ in Eq. (16). 


\subsubsection{Delamination failure of the struts}

The composite struts can fail also by compressive delamination. The shear strength with compressive delamination as the failure mode is given by Equation (4.10) with $\sigma_{c}$ replaced by the delamination strength $\sigma_{d l}$ of the composite struts. In the case of vanishing node volume, the modified Equation (4.10a) reduces to:

$$
\frac{\tau_{p}}{\sigma_{d l}}=\bar{\rho} \sin 2 \omega\left[1+\frac{\bar{\rho} \sin ^{3} \omega}{2}\right] \sqrt{1+\tan ^{2} \alpha},
$$

With an analogous expression for $\pi / 4<\alpha \leq \pi / 2$ given by replacing $\cos \alpha$ by $\sin \alpha$ in Equation (4.16).

\subsubsection{Euler buckling of the struts}

Under shear loading, the pyramidal core may collapse by the elastic buckling of the constituent struts. Recall that the Euler buckling stress of an end-clamped strut subjected to an axial load is given by:

$$
\sigma_{E}=\frac{\pi^{2} E_{s} t^{2}}{3 l^{2}},
$$

and thus the shear strength of the pyramidal core due to the elastic buckling of the constituent struts is given by replacing $\sigma_{c}$ by $\sigma_{E}$ in Equation (4.10) and replacing $\sigma_{d l}$ by $\sigma_{E}$ in Equation (4.16) for the case of vanishing node volume.

Delamination failure of the struts can be prevented in appropriately designed composite pyramidal cores. In order to illustrate the optimal performance of the composite pyramidal cores, the predicted normalized peak strength of the composite pyramidal core $\tau_{p} /\left(\bar{\rho} \sigma_{\max }\right)$ is plotted in Figure 4.3 as a function of relative density $\bar{\rho}$ 
only considering the micro-buckling and Euler buckling failure mechanisms of the struts. Predictions are shown in Figure 4.1 for three selected values of $\bar{E}_{s} \equiv E_{s} / \sigma_{\max }$ representative of unidirectional $\left(\bar{E}_{s}=167\right)$, laminated $\left(\bar{E}_{s}=116\right)$ and woven $\left(\bar{E}_{s}=50\right)$ carbon fiber composites. For the purposes of illustration, in Figure 4.3 the volume of the nodes is neglected, with the choices $\alpha=45^{\circ}, \omega=45^{\circ}$ and $\bar{\phi}=2^{\circ}$. The misalignment angle $\phi=2^{\circ}$ is consistent with experimental evidence for unidirectional materials ${ }^{51}$. The normalized strength $\tau_{p} /\left(\bar{\rho} \sigma_{\max }\right)$ is a measure of the efficiency of the

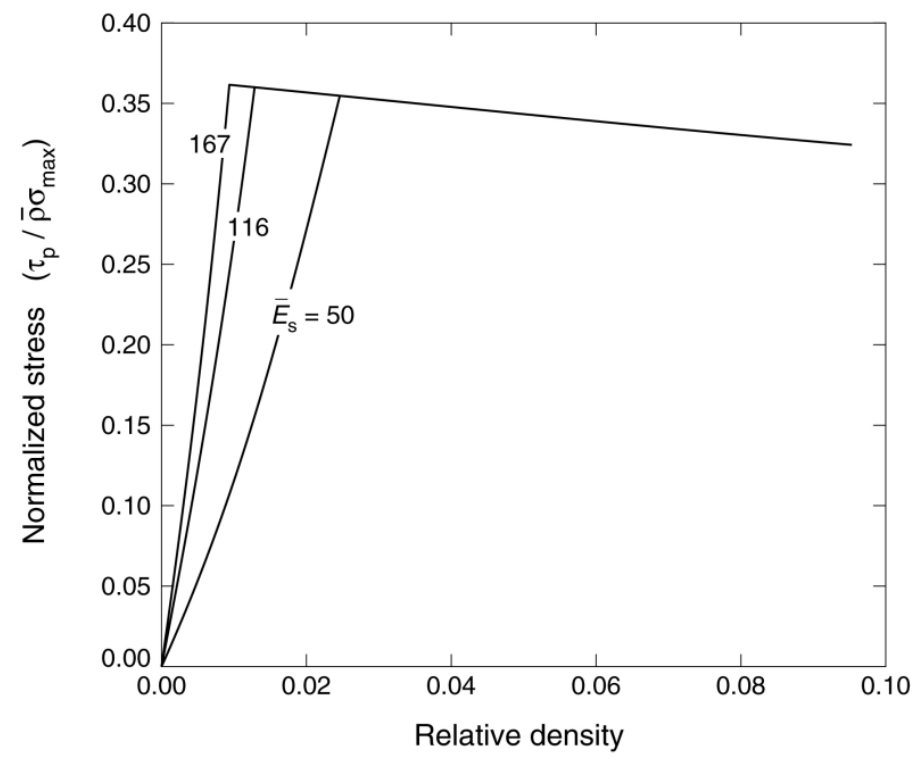

Figure 4.3: Predictions of the variation of the normalized peak strength $\tau_{p} /\left(\bar{\rho} \sigma_{\max }\right)$ with relative density $\bar{\rho}$ for three selected value of the normalized laminate modulus $\bar{E}_{s}$. The predictions assume that the node volume is negligible. 
topology in terms of its structural strength. Since $\tau_{p} /\left(\bar{\rho} \sigma_{\max }\right)=1$ corresponds to a cellular material that attains the Voigt upper bound, we expect $\tau_{p} /\left(\bar{\rho} \sigma_{\max }\right) \leq 1$. We note:

(a) The normalized strength $\tau_{p} /\left(\bar{\rho} \sigma_{\max }\right)$ peaks at a $\bar{\rho}$ value at which the failure modes transition from Euler buckling to micro-buckling. Designs at this transition value of $\bar{\rho}$ are most efficient in terms of their strength to weight ratio. This is rationalized by noting that in the Euler buckling regime the structural efficiency increases with increasing $\bar{\rho}$ as the struts become more stocky resulting in an increase in their Euler buckling loads. By contrast in the micro-buckling regime with increasing $\bar{\rho}$, the shear forces on the struts increase resulting in a decrease in their micro-buckling stress as per Equation (4.7).

(b) The maximum value of $\tau_{p} /\left(\bar{\rho} \sigma_{\max }\right)$ for the pyramidal cores increases with increasing $\bar{E}_{s}$ with the transition from Euler buckling failure to microbuckling then occurring at lower values of $\bar{\rho}$.

\subsection{Comparison with measurements}

The measurements detailed in Chapter 3 are subsequently compared with the analytical predictions detailed above. In making these predictions we employ the following material properties for the composite struts consistent with the measurements discussed in Chapter 3 and tabulated in Table 3.1: (i) Young's modulus $E_{s}=28 \mathrm{GPa}$, 
(ii) the delamination failure strength $\sigma_{d l}=380 \mathrm{MPa}$, (iii) the micro-buckling strength $\sigma_{\max }=615 \mathrm{MPa}$ and (iv) misalignment angle $\bar{\phi}=2^{\circ}$. The strut angle is taken to $\omega=45^{\circ}$ in all cases. The geometric dimensions of each relative density of the pyramidal cores are listed in Table 3.2- these values are used to determine the values of $\bar{l}$ and $\bar{b}$ required in the analytical expressions detailed in this chapter.

\subsubsection{Shear loading at $\alpha=45^{\circ}$}

The analytical predictions of the shear modulus are included in Figure 4.4a and agree well with the measurements over the range of relative densities investigated here. The elastic modulus of the lattice scales approximately linearly with the relative density, in line with the expectation for a periodic lattice. The predictions of peak strength are included in Figure $4.4 \mathrm{~b}$ with the predicted failure modes indicated (recall that the operative failure mode for a given relative density and set of material properties is the mode that gives the lowest peak strength). Three samples were tested for each relative density, and the average measurement for each relative density is shown in Figure 4.4, along with the range of the measurements. The measured and predicted values of the peak strengths are in good agreement. Moreover, in line with the observations discussed in Chapter 3, the predicted failure mode is delamination expect for the $\bar{\rho}=0.9 \%$ specimen that lies at the boundary between the Euler buckling and delamination failure modes. 

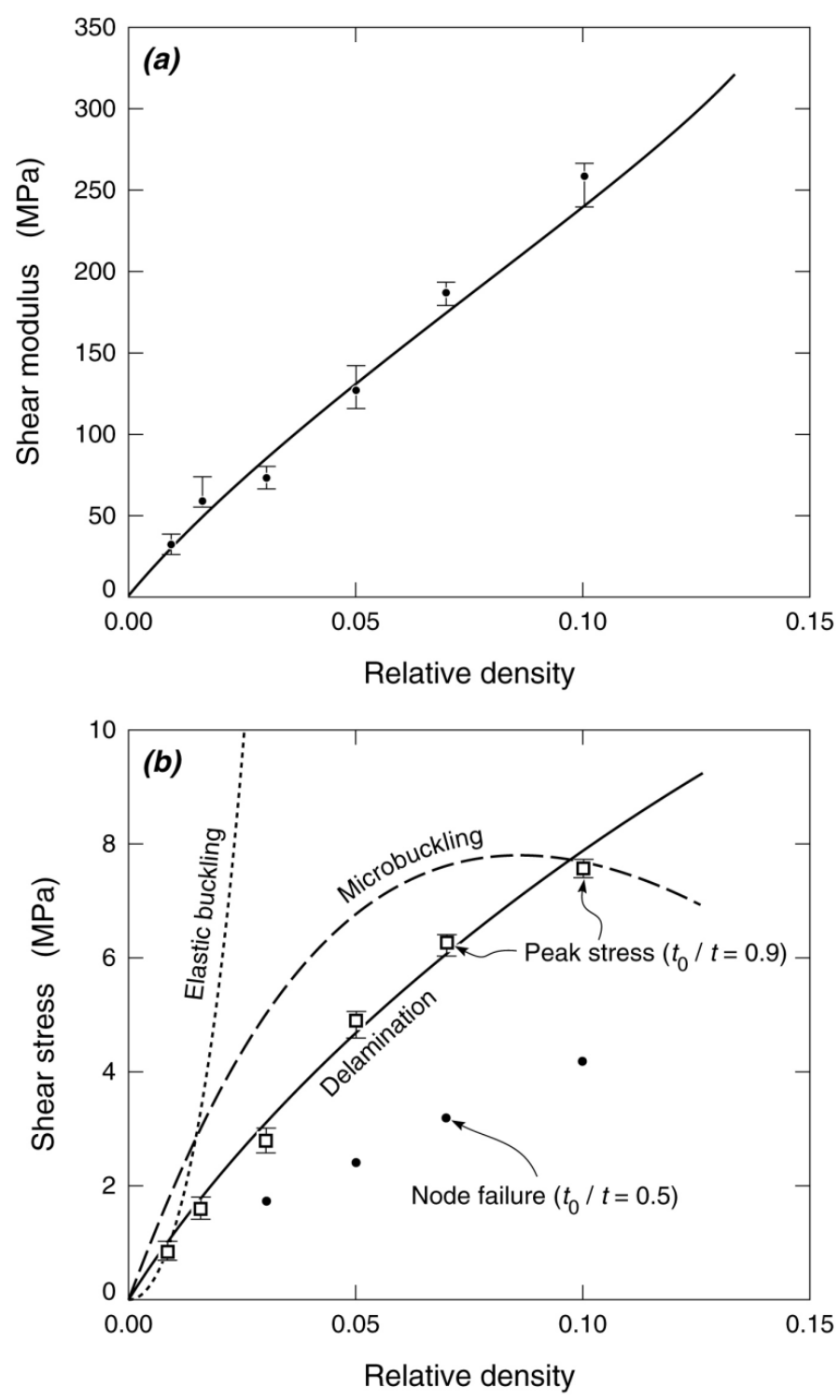

Figure 4.4: Summary of the measured (a) unloading modulus and (b) peak strength as a function of relative density $\bar{\rho}$ for shear loading at $\alpha=45^{\circ}$ (node thickness $t_{0} / t=0.8$ ). The lines are predictions of the micromechanical models $\left(t_{0} / t=0.8\right)$. 


\subsubsection{Effect of shearing angle $\alpha$}

The analytical predictions indicate that the shear modulus is independent of the shearing angle $\alpha$. This is borne out by the measured $\tau$ versus $\gamma$ responses included in Figure 3.9 wherein the elastic regime slopes are essentially independent of $\alpha$. The predictions of the peak strengths are also included in Figure 3.9. The predictions are generally in line with the measurements, suggesting that the shear strength decreases with decreasing $\alpha$.

\subsection{Comparison with competing materials}

The measured shear modulus and peak shear strengths of the CFRP pyramidal cores are included in Figures 4.3a and 4.3b, respectively along with a number of competing materials and topologies. In this Ashby ${ }^{10}$ plot the density of the pyramidal cores is taken to be $\rho=\bar{\rho} \rho_{s}$ with $\rho_{s}=1440 \mathrm{kgm}^{-3}$. The CFRP pyramidal cores have a performance similar to CFRP honeycombs in terms of both shear strength and modulus. The investigation by Finnegan et $\mathrm{al}^{4}$ has indicated that these CFRP pyramidal cores also have a similar compressive performance to CFRP honeycombs. Thus, for a range of multi-functional applications that require an "open-celled" architecture (e.g. so that cooling fluid can pass through a sandwich core), the CFRP pyramidal cores offer an attractive alternative to honeycombs. 

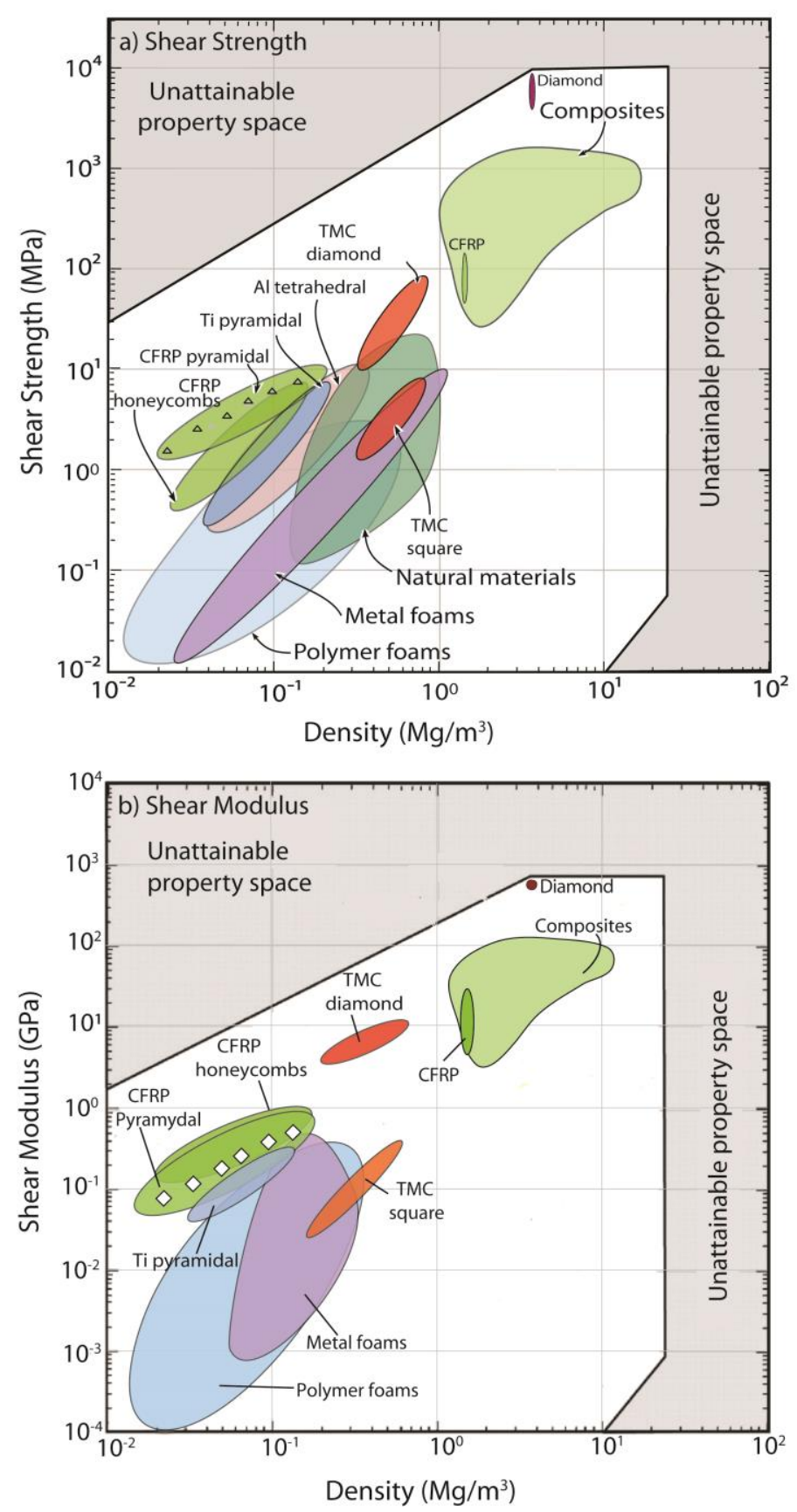

Figure 4.5: An Ashby ${ }^{10}$ chart of (a) shear modulus and (b) shear strength as a function of density. In addition to a range of competing cellular materials, properties of the CFRP pyramidal cores measured in this study are included. 


\subsection{Laminated structure advantages and limitations}

Pyramidal truss sandwich cores with relative densities $\bar{\rho}$ in the range $1-10 \%$ have been manufactured from 0/90 crossply carbon fiber reinforced polymer laminates by employing a snap-fitting method. The measured quasi-static shear strength varied between 1 and $7.5 \mathrm{MPa}$; increasing with increasing $\bar{\rho}$. Two failure modes were observed: (i) Euler buckling of the struts and (ii) delamination failure of the struts.

Analytical models are developed for the elastic response and collapse strengths of the composite cores. In general good agreement between the measurements and predictions is obtained. Along with the complimentary work ${ }^{4}$, the last two chapters demonstrate that composite cellular materials with a pyramidal micro-structure fill a gap in the strength versus density material property space and compete favorably with honeycomb designs. However, current designs of the pyramidal cores have not optimized the node designs and thus use material rather inefficiently. Moreover, the current designs undergo delamination failure of the struts and thus do not achieve the full potential of composite cores as predicted by the micro-buckling analysis presented here. A fabrication method which suppresses delamination would potentially enhance the strength of these structures. Chapter 5 explores one such approach. 


\section{Chapter 5}

\section{Hybrid CFRP/Foam Structures}

The compressive strength of CFRP lattice structures made from laminates is governed by elastic buckling of the struts at low relative densities, or truss delamination (inter-ply splitting). In shear, the strength of the adhesive used to attach the truss to the face sheet is also a limiting factor. In addition, the use of $0^{\circ} / 90^{\circ}$ laminates results in at most only half of the fibers being oriented in the direction of the load applied to the truss. The strength of CFRP truss structures could therefore be increased by: (i) increasing the fraction of fibers aligned in the loading direction, (ii) creating trusses better able to withstand interplay delamination failure, and (iii) developing a more robust node-face sheet bonding method. However, once a brittle CFRP strut failure occurs, the remnant strength of the lattices would be low, and so a CFRP core structure might be ill-suited for impact energy absorption applications.

In this chapter, the use of a braided carbon fiber approach for fabricating CFRP pyramidal lattice structures that reinforce closed cell polymer foams in a hybrid CFRP truss/foam core sandwich panel is explored. The braided trusses are non-laminated materials. In principle this eliminates the delamination failure mode. In addition, all the fibers are aligned within a few degrees of the braid axis which may increase the axial compressive strength of the strut. This chapter investigates the fabrication of a braided truss structure and the effect of varying the foam strength of a hybrid pyramidal truss/foam core upon mechanical response in compression and shear. 


\subsection{Panel Design and Fabrication}

\subsubsection{Design Concept}

The sandwich panel concept explored here is schematically illustrated in Figure 5.1. The panel cores were assembled from a braided carbon fiber net and prismatic, closed cell polymer foam inserts to form a hybrid CFRP pyramidal lattice/foam core structure. The core struts were Kevlar fiber stitched to 3D woven carbon fiber face sheets, and the structure then infused with an epoxy resin and cured. The closed cell polymer foam inserts served a number of purposes. They (i) provided a means of supporting and defining the cross-sectional shape of the trusses, (ii) maintained uniform face sheet separation, (iii) increased the area of adhesively bonded interface with the faces and (iv) provided core strengthening and impact energy storage during subsequent compression and shear loading.

A variety of foams were used to investigate the effect of foam strength (at the cost of increased density) on the hybrid cellular materials mechanical properties. Table 5.1 summarizes the densities and mechanical properties of the foams used in the study. They included closed cell PVC Divinycell (Diab Inc.) foams with densities of $80-250 \mathrm{kgm}^{-3}$ and compressive strengths of 1.6-6.8 MPa, and syntactic foams (UDC Corporation) consisting of hollow glass spheres in a polymer matrix with densities of $320-448 \mathrm{kgm}^{-3}$ and compressive strengths of 10-26 MPa. A very weak, but easily removed polyurethane foam was also used to fabricate samples so that an empty lattice mechanical response could be measured. The foams were $\mathrm{CNC}$ milled to create trapezoidal cross-section prisms with semicircular, $2.25 \mathrm{~mm}$ radius grooves to contain the carbon fiber braided lattice within a $4.5 \mathrm{~mm}$ diameter channel. 


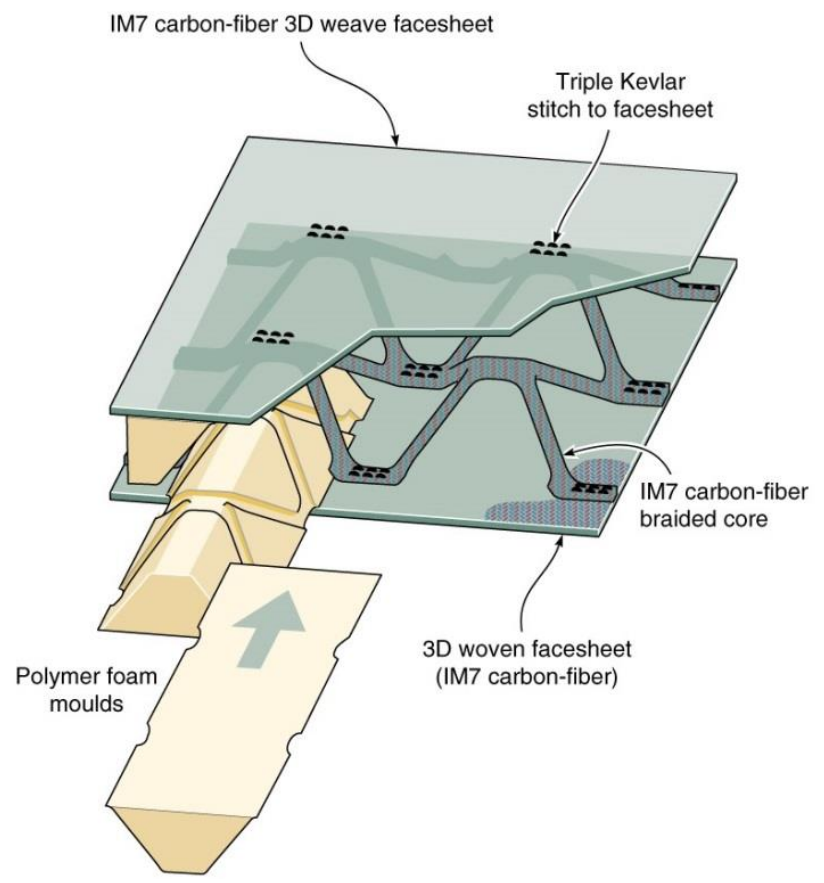

Figure 5.1: Hybrid composite core structure consisting of a braided CFRP pyramidal lattice with polymer foam inserts configured as the core of a sandwich panel with 3D woven carbon fiber composite face sheets. 
Table 5.1: Summary of foam properties

\begin{tabular}{|l|c|c|c|c|c|}
\hline \multicolumn{1}{|c|}{ Material } & $\begin{array}{c}\text { Density } \\
\left(\mathrm{kg} / \mathrm{m}^{3}\right)\end{array}$ & $\begin{array}{c}\text { Compressive } \\
\text { Strength } \\
(\mathrm{MPa})\end{array}$ & $\begin{array}{c}\text { Shear } \\
\text { Strength } \\
(\mathrm{MPa})\end{array}$ & $\begin{array}{c}\text { Compressive } \\
\text { Modulus } \\
(\mathrm{MPa})\end{array}$ & $\begin{array}{c}\text { Shear } \\
\text { Modulus } \\
(\mathrm{MPa})\end{array}$ \\
\hline $\begin{array}{l}\text { Polyurethane } \\
\text { foam }\end{array}$ & 32 & 0.172 & 0.152 & 14 & 5 \\
\hline Divinycell H80 & 80 & 1.3 & 0.95 & 85 & 23 \\
\hline Divinycell H100 & 100 & 2 & 1.4 & 115 & 28 \\
\hline Divinycell H130 & 130 & 2.7 & 1.9 & 145 & 40 \\
\hline Divinycell H200 & 200 & 4.2 & 3.2 & 265 & 65 \\
\hline Divinycell H250 & 250 & 6.8 & 3.9 & 350 & 81 \\
\hline Synfoam H20 & 320 & 10 & 6.4 & 360 & 110 \\
\hline Synfoam H24 & 384 & 16 & 9.8 & 460 & 125 \\
\hline Synfoam H28 & 448 & 26 & 14.2 & 575 & 140 \\
\hline
\end{tabular}

\subsubsection{Braided net fabrication}

Three dimensional braiding ${ }^{52,53,54,55,56}$ was used to create a braided carbon fiber net in which the trusses contained multidirectional fiber reinforcements whose angle of deviation from the longitudinal axis of the truss was up to $11^{\circ}$. The principal advantages of 3-D braided preforms are that (i) they can be fabricated in various complex (and, if desired continuously variable) shapes; (ii) their shear and torsional rigidities are significantly higher than those of a traditional laminated structure and (iii) the orientation of the filaments can be controlled. The fiber volume fraction in 3-D braided composite 
can also be widely varied, depending on the requirements for matrix infiltration, consolidation and densification of the various resin types.

The 3BRAID® process used here was developed at 3Tex and involved three machine motions ${ }^{54}$. Figure 5.2(a) shows a schematic of the single 64 carrier braiding module used here. Each of the 16 large circles represents a horn gear occupied by 4 braiding carriers colored in gray, while the smaller circles represent the fork gears. All fork gears in this application are active and so colored green. The horn gear ${ }^{57}$, which contained 4 yarn carriers, first completed a $1 \frac{1}{4}$ turn. Fork gears, located at the interstices of the horn gears then switch yarn carriers between horn gears by completing a $1 / 2$ turn. Lastly, a take-up system advanced the braid by a predetermined distance.
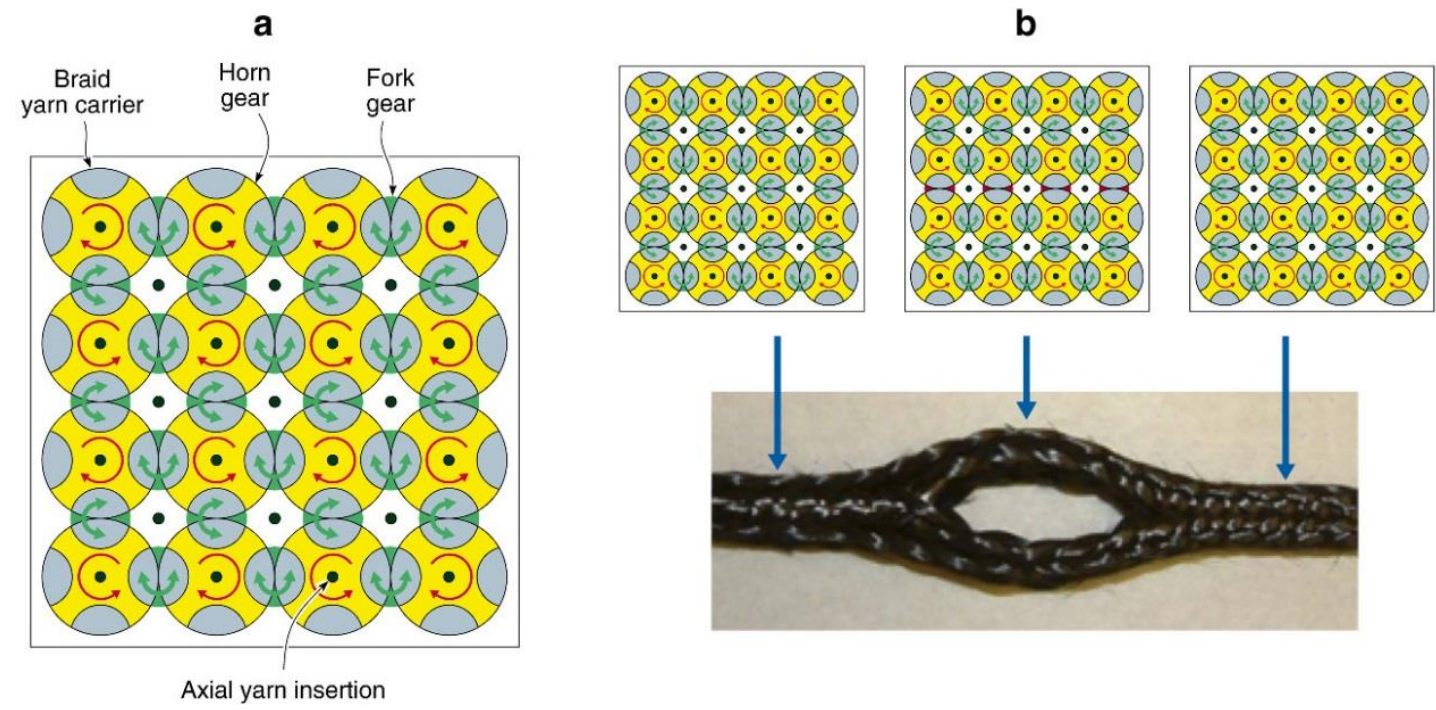

Figure 5.2 (a) Schematic of 64 carrier single module used for 3D braiding. (b) Shape control in the 3BRAID® process; disengagement of fork gears (the color red indicates which gears are disengaged) causes the preform to split. Reengagement of the fork gears rejoins the preform. 
Three methods can be used to control the shape of the preform in the 3BRAID® process. These include selective engagement or disengagement of individual fork gears, arrangement of the braiding carriers on the base of the machine, combined with selective control of individual fork gears, and tailored placement of axial yarns. Selective engagement of individual fork gears controls the exchange of braiding carriers between horn gears, which can be used to define the formation of internal openings within the preform, as well as the outside surfaces of the preform.

In Figure 5.2(b), the green colors mark fork gears where an exchange will occur and red colors mark fork gears where no exchange will occur. In this example, a single square shaped preform, formed by engaging all of the horn gears and fork gears, is split into 2 small rectangular shapes formed by disengaging the center fork gears during the several machine cycles, and then rejoined by reengaging the center fork gears. By alternating the splitting and joining from one section of the braid pattern to another, a truss braid or "web" architecture could be produced. Production of the split and joined sections occurred continuously as the 3D braided preform was produced.

The braided net used here was constructed from 24 tows of $12 \mathrm{~K}(12,000$ fibers per tow), Hexcel IM7 carbon fiber. The IM7 fiber has tensile strength of 5.67 GPa, a modulus of $276 \mathrm{GPa}$, and a density of $1800 \mathrm{kgm}^{-3}$. Figure $5.3(\mathrm{a})$ defines the structural parameters of this braid, while Figure 5.3(b) shows a section of the braid used here. The braid angle, $\phi=11^{\circ}$, its diameter, $d=4.5 \mathrm{~mm}$ and the repeat distance, $\lambda=25 \mathrm{~mm}$. The braided net had a density of $442 \mathrm{kgm}^{-3}$ and its unit cell geometry is defined in Figure 3(c). 
a Braid structure

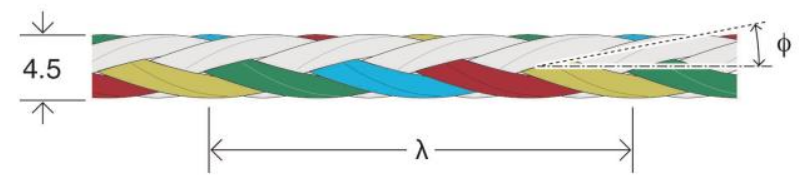

b IM7 braid

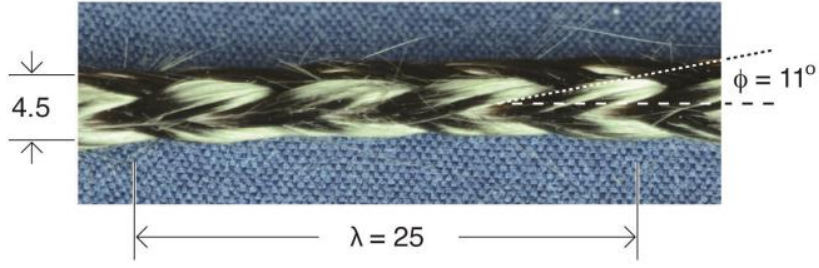

C Braided Net

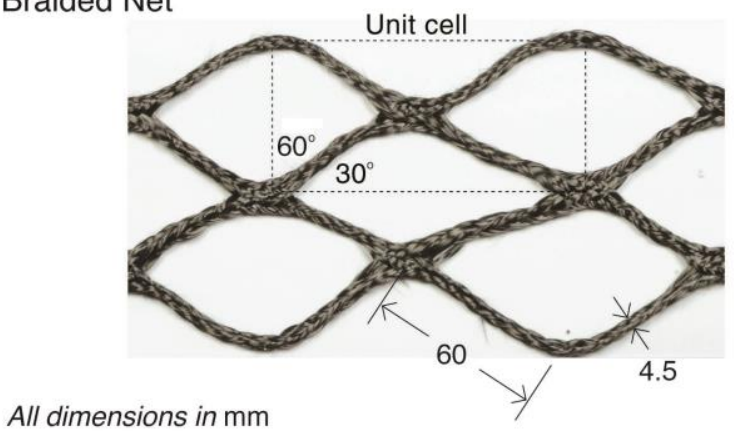

Figure 5.3 (a) Schematic illustration of the structure of the braided carbon fiber strut. (b) Photograph of a strut section of the braided net. c) The braided net structure, with unit cell shape and dimensions identified.

\subsubsection{Dry Panel Assembly}

The dry assembly of a hybrid foam/CFRP truss core sandwich panel is schematically illustrated in Figure 5.4. The braid was first stitched to the dry face sheet, Figure 5.4(a). It was then placed within the $2.25 \mathrm{~mm}$ radius grooved channels of a foam insert, Figure 5.4(b), and an inverted foam mold was inserted to enclose the braided truss within a $4.5 \mathrm{~mm}$ diameter channel, Figure 5.4(c). The braid was then stitched to the lower face sheet and the process repeated to form a pyramidal structure, Figure 5.4(d). The 
braid- face sheet nodes were reinforced using three stitches of Kevlar thread each approximately $15 \mathrm{~mm}$ in length. The $3 \mathrm{Weave}^{\mathrm{TM}}$ face sheets were made from $12 \mathrm{~K}$ Hexcel IM7 Carbon fibers at 3Tex Inc. The sheet consists of fiber tows running in the $\mathrm{x}, \mathrm{y}$ and $\mathrm{z}$ directions, with $43 \% \mathrm{x}$-fibers (warp), $47 \% \mathrm{y}$-fibers (weft), and $10 \%$ z-fibers. The sheets had a thickness of $3.5 \mathrm{~mm}$, prior to infusion, and an areal density of $2.08 \mathrm{kgm}^{-2}$. The 3D woven structure of both the braid and face sheet were selected to increase resistance to delamination.

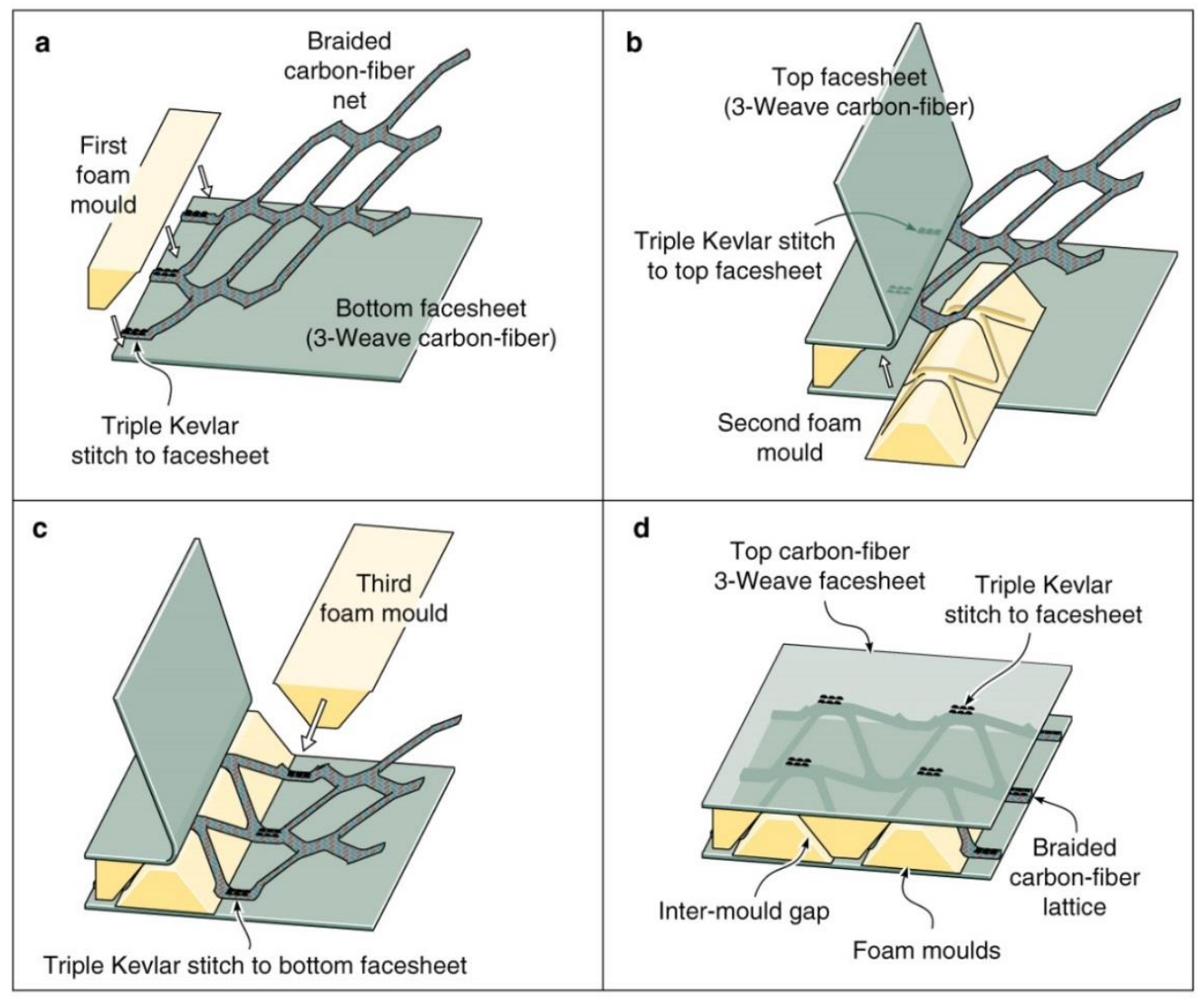

Figure 5.4: The hybrid CFRP pyramidal lattice core sandwich panel assembly sequence. 


\subsubsection{Resin Infusion Process}

A SC1A grade epoxy resin with SC1B curing agent (Applied Poleramic Inc., Benicia, CA) was used for the polymer matrix. This epoxy was selected because of its $(i)$ low viscosity $\left(295 \mathrm{cps}\right.$ at room temperature $\left.23^{\circ} \mathrm{C}\right)$, which allowed complete infiltration of the complex shaped panel during the infusion process, (ii) moderately high strength, and (iii) high impact resistance. The cured epoxy has a compressive strength of $75 \mathrm{MPa}$, and a Young's modulus $E_{\text {resin }}=1.85 \mathrm{GPa}$. A vacuum assisted resin transfer molding (VaRTM) process was used to infuse the assembled carbon fiber structures. The setup prior to infusion is illustrated in Figure 5.5. The infusion and cure cycle were performed in an autoclave, which enabled control of the temperature and pressure (vacuum) within the assembly throughout the process. The samples were infused on a wax coated glass substrate to enable removal of the panel after infusion. A layer of breather material and a peel ply were then laid over the wax covered glass. The carbon fiber panel was then placed on top of the peel ply. A second layer of peel ply was used to cover the panel, and a layer of distribution media was used to ensure even flow of resin throughout the part. Another layer of breather material was placed over the distribution media, and finally, a nylon vacuum bag was used to enclose and seal the part. Inlet and outlet tubes were inserted in the vacuum bag. The outlet tube was connected to a resin trap, and could be separately evacuated from the autoclave. The inlet tube was connected to the resin container.

The panels were infused using an epoxy to curing agent ratio of 100:22. The vacuum bagged parts were placed in the autoclave, and the outlet line evacuated to a 
vacuum pressure $P_{v}=-3.6 \mathrm{kPa}$, with the resin inlet line sealed. The inlet line was then opened, and resin allowed to flow through the part and began to exit through the outlet tube. The inlet tube was then closed, and the resin cure cycle program was executed within the autoclave. The part was first externally pressurized to $0.1 \mathrm{MPa}$, while maintaining the internal vacuum pressure. The vacuum line was then released to atmospheric pressure, and the external pressure increased to $0.17 \mathrm{MPa}$ to eliminate resin vapor voids. The part was then heated up to $71^{\circ} \mathrm{C}$, and the pressure and temperature maintained for a 4 hour curing period.

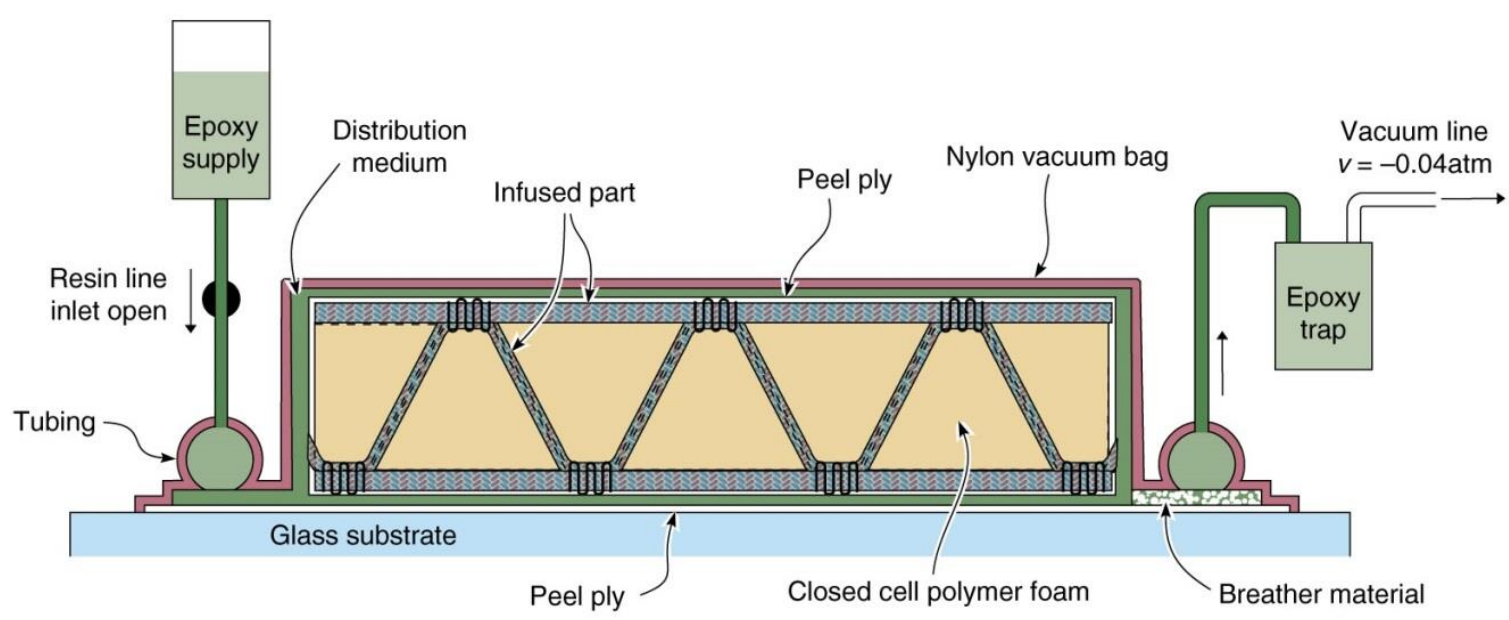

Figure 5.5: Setup used for the vacuum assisted epoxy resin infusion process.

After cure, the panels were removed from the infusion tooling and machined to the appropriate dimensions needed for testing. Each compression test panel comprised of four unit cells, in a $2 \times 2$ unit cell array. The shear test panels were 6 unit cells long and two unit cells wide. The foam was mechanically removed from some samples in order to ascertain the empty lattice mechanical response. We note that small gaps between the prismatic foam inserts resulted in a thin layer of resin being retained in the hybrid core 
panels. Figure 5.6 shows a photograph of a finished hybrid core panel, as well as an x-ray computed tomographic (X-CT) reconstruction. The retained corrugated resin layer can be clearly seen in the reconstructed image. The X-CT characterization also revealed that the trusses had an elliptical cross section. This was due to deformation of the foam during the consolidation process, and this is discussed more extensively below.
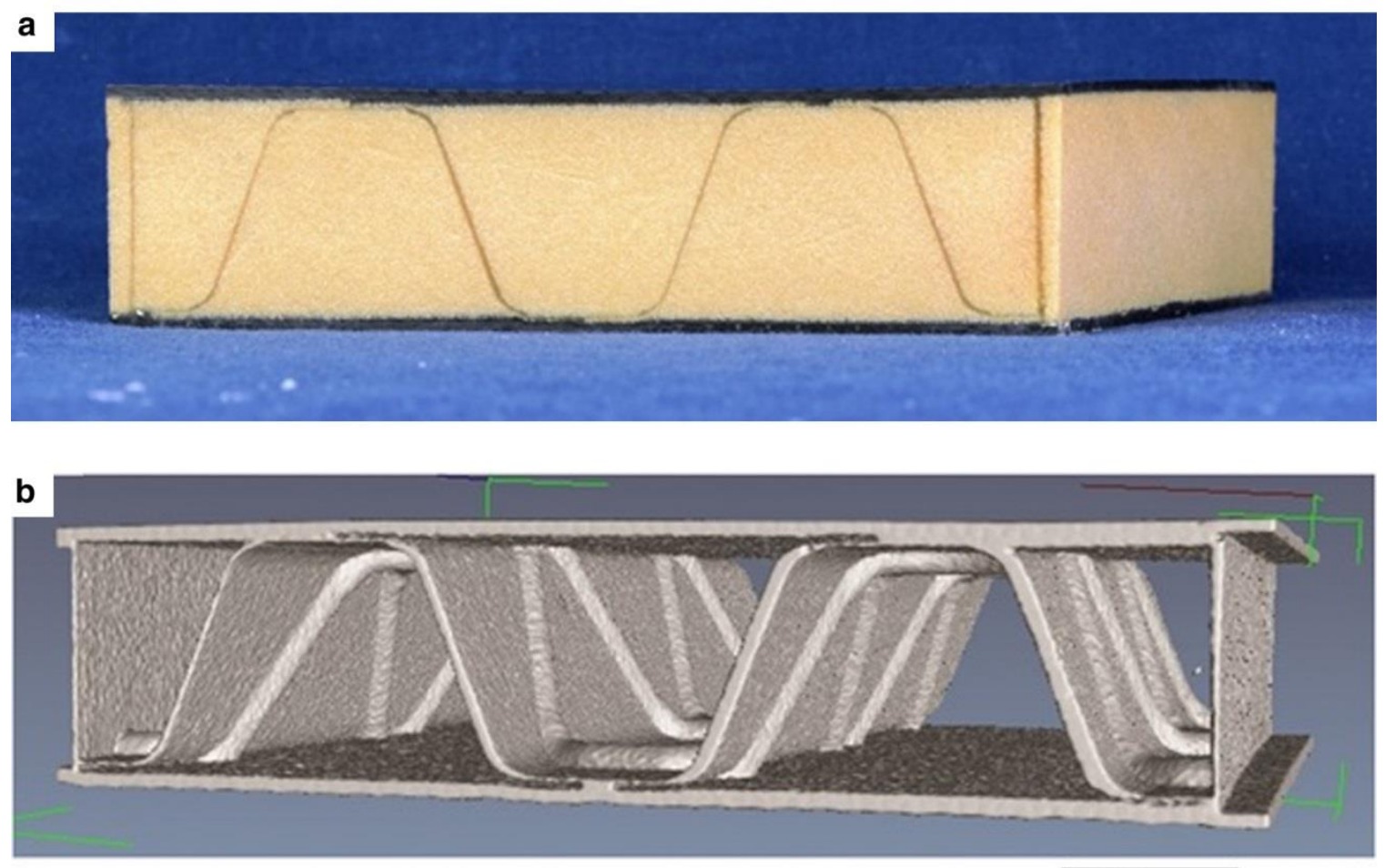

$20 \mathrm{~mm}$

Figure 5.6 a) Photograph of a completed hybrid panel with CFRP pyramidal lattice and H250 Divinycell foam core. b) X-CT image of the sample shown in (a) revealing the interior structure of the finished panel with the foam digitally filtered out. 


\subsubsection{Core Geometry}

A unit cell of the pyramidal lattice is shown in Figure 5.7 with elliptical cross section trusses with a minor axis width $d_{1}$ and major axis length $d_{2}$. The angle of inclination of the trusses to the base of the unit cell $\omega=54^{\circ}$. The truss-face sheet node in both cases was assumed to have a width equal to the major axis diameter of the truss, and a length $b$ that was set equal to 2 times the woven diameter of the braid $(d=4.5 \mathrm{~mm})$, maintaining the woven node dimensions. The length of the truss, $l=41 \mathrm{~mm}$.

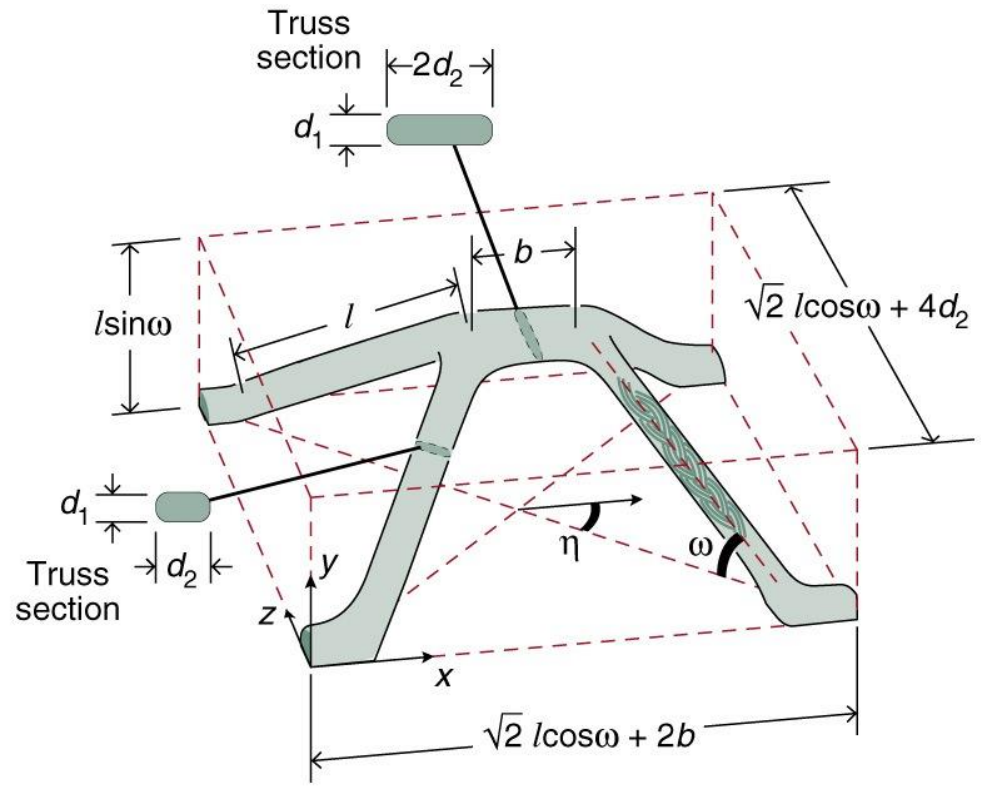

Figure 5.7: Pyramidal CFRP unit cell with elliptical cross section trusses. The minor axis of the truss cross-section was perpendicular to the trapezoidal side face of the foam core (not shown) used to support the truss. 
The relative density of the CFRP empty lattice unit cell $\bar{\rho}$ was found by calculating the ratio of the truss volume to that of the unit cell. One quarter of a node can be assigned to each lower corner of the unit cell, and a full node volume to the top face. The truss volume within the unit cell includes the node volume, and for the most general case of an elliptical truss:

$$
\bar{\rho}=\frac{\pi d_{1} d_{2}(l+b)}{l \sin \omega\left(\sqrt{2} l \cos \omega+4 d_{2}\right)(\sqrt{2} l \cos \omega+2 b)}
$$

In the special case where a truss was not deformed during resin infiltration, $d_{1}=d_{2}=d$. The measured values of $d_{1}$ and $d_{2}$ were determined from X-CT reconstructions of the as fabricated samples, Table 5.2. These measurements were taken along orthogonal crosssectional slices of the trusses.

The total density of the hybrid core, $\rho$, is found by accounting for the masses of the foam and resin sheets. The volume, $v_{r s}$ occupied by a resin sheet of thickness $t$ within the unit cell was estimated by calculating the volume of the corrugated resin sheet:

$$
v_{r s}=2 t(l+b)(\sqrt{2} l \cos \omega+4 d)
$$

The volume fraction of the resin sheet $v_{f r}$ is found by dividing Equation (6a) by the volume of the unit cell: 


$$
v_{f r}=\frac{2 t(l+b)}{l \sin \omega(\sqrt{2} l \cos \omega+2 b)}
$$

The thickness of the resin sheet varied from 0.1 to $1.0 \mathrm{~mm}$ and had an average value $t_{\text {avg }} \approx 0.5 \mathrm{~mm}$ for all specimens tested. For this value of $t, v_{f r} \approx 0.025$. This value is used for subsequent calculations. It was assumed that the volume within the unit cell which was not occupied either by the truss or the resin sheet was occupied by foam. The volume fraction of the foam $v_{f f}$ can then be estimated as:

$$
v_{f f}=\left(1-\left(\bar{\rho}+v_{f r}\right)\right)
$$

The total density of the hybrid core, $\rho$ can then be expressed as:

$$
\rho=\left(\bar{\rho} \rho_{c c}\right)+\left(\rho_{r s} v_{r s}\right)+\left(\rho_{f} v_{f f}\right)
$$

where, $\rho_{c c}$ is the density of the braided CFRP trusses $\left(1450 \mathrm{kgm}^{-3}\right), \rho_{r s}$ is that of the cured epoxy resin $\left(1100 \mathrm{kgm}^{-3}\right)$, and $\rho_{f}$ is the density of the foam given in Table 2 . 


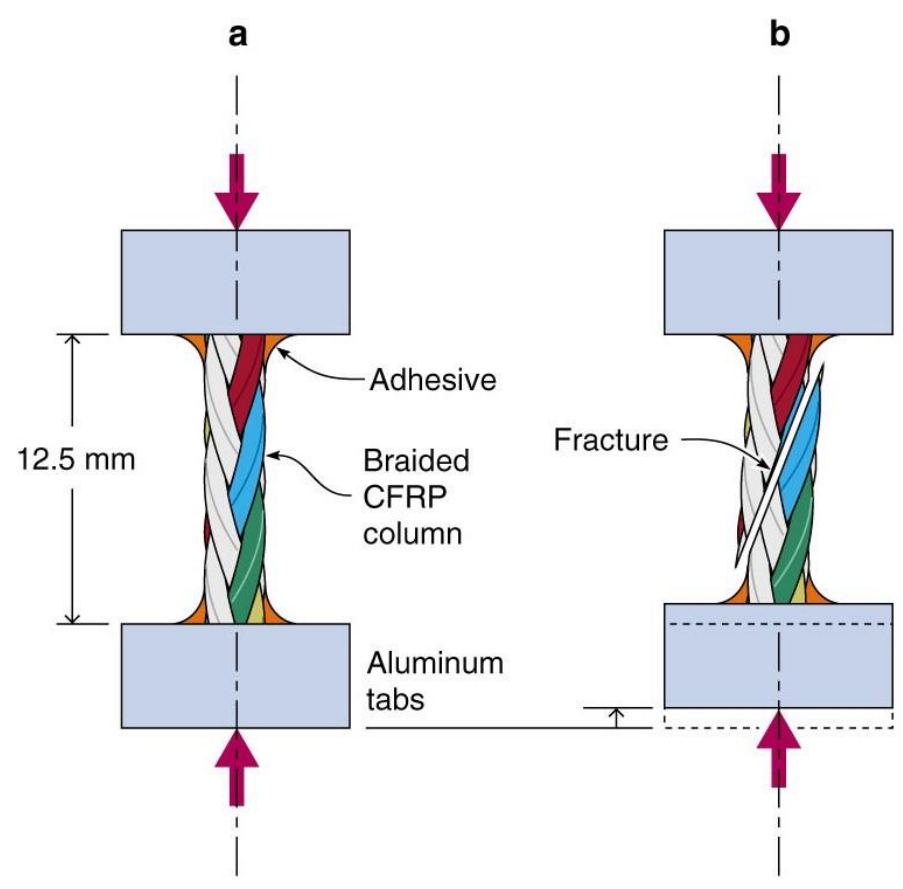

Figure 5.8: (a) Schematic illustration showing setup used to determine compressive strength of the braided composite strut, and (b) the orientation of the strut fracture surface.

\subsection{Braided CFRP Truss Properties}

\subsubsection{Compression}

The compressive strength of a circular cross section CFRP braid was first determined. Samples were prepared by flowing resin through a length of the braid, andconfined within a circular glass tube with an inner diameter $d=4.5 \mathrm{~mm}$ equal to that of the braid. The resin was cured, and the tubing removed, leaving behind the cylindrical braided composite sample with a fiber volume fraction of $52 \%$. These samples were cut into $12.5 \mathrm{~mm}$ long pieces, and their ends coated with SCI epoxy to prevent brooming failure $^{58}$ and glued to a pair of platens. They were then tested in compression as shown in 
Figure 5.8(a) taking care to ensure the two platens were parallel, and the cylindrical axis of the CFRP braids was normal to the platens. The samples were tested in compression at ambient temperature $\left(23^{\circ} \mathrm{C}\right)$ at a strain rate of $2 \times 10^{-4} \mathrm{~s}^{-1}$.

The compressive stress-strain response of a typical sample is shown in Figure 5.9(a). The average compressive strength for 5 tests $\sigma_{\max }=540 \pm 40 \mathrm{MPa}$, and the Young's modulus (measured during unloading) $E_{\text {truss }}=28 \pm 2 \mathrm{GPa}$. The braided CFRP struts failed macroscopically in shear, Figure 5.8(b). Figure 5.9(b) shows an XCT cross section of a failed truss. This scan was done using an XRADIA MicroXCT200 X-ray computed tomography system. It can be seen that the initial failure occurred by microbuckling within one of the tows. This occurred on a plane at approximately $45^{\circ}$ to the loading axis and displaced the ends of the tow in the struts radial direction. This resulted in cracks forming in adjacent tows and macroscopic failure propagating at an angle to the direction of applied load. 


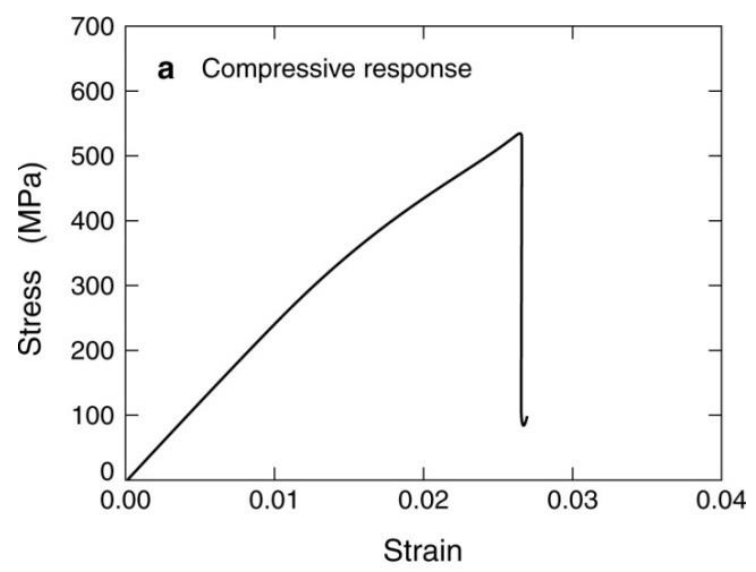

b X-CT image of strut mid-plane at failure
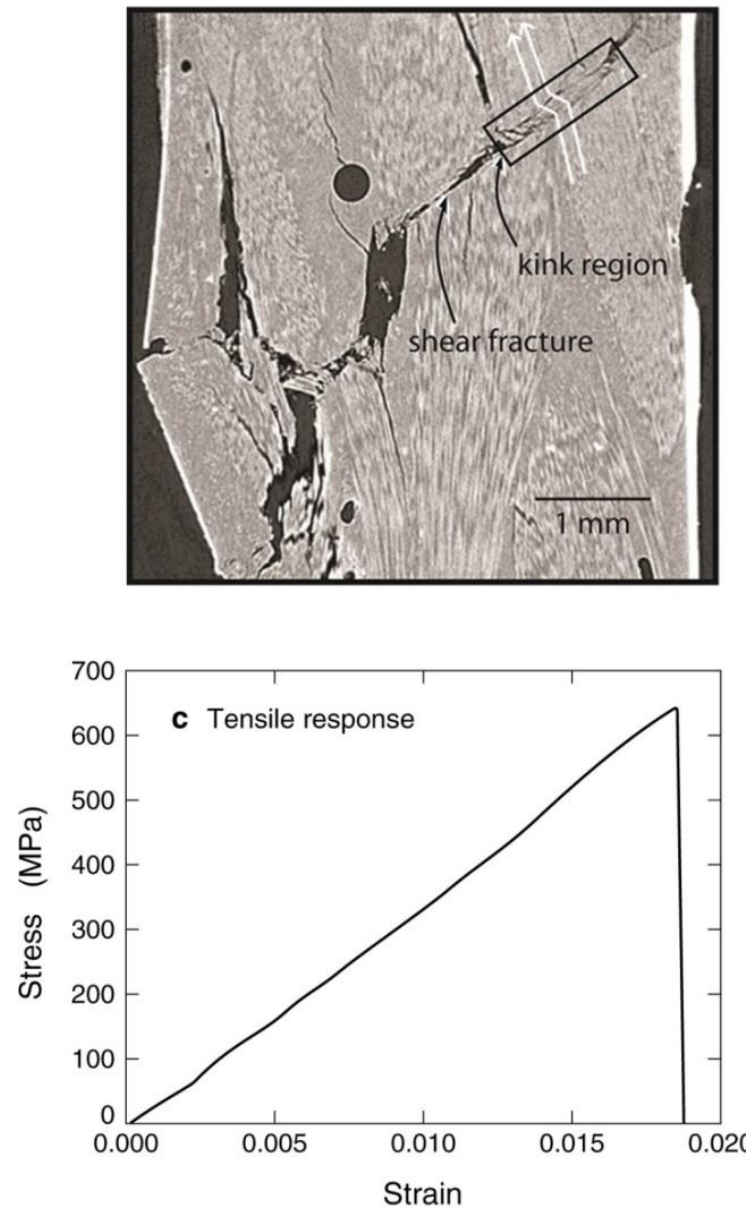

Figure 5.9: (a) Axial compressive stress-strain response of a braided truss. (b) X-CT cross sectional image of a failed truss specimen showing damage initiating by microbuckling in one of the tows and shear crack propagation across the truss. (c) Axial tension test response of a braided truss. 


\subsubsection{Tension}

The tensile strength of the truss was determined in accordance with the ASTM D3039 standard for composite tensile testing. The samples were made by the same method as the samples for compressive testing (with $d=4.5 \mathrm{~mm}$ ). The samples are securely clamped within the grips and had a $50.8 \mathrm{~mm}$ gauge length. The samples were then strained at a constant rate of $2 \times 10^{-4} \mathrm{~s}^{-1}$, at ambient room temperature $\left(23^{\circ} \mathrm{C}\right)$. A typical stress - strain response is shown in Figure 5.9(c). The trusses are found to have an average tensile strength (from 5 tests) of $\sigma_{\text {tensile }}=640 \pm 30 \mathrm{MPa}$, and a Young's modulus (measured during unloading) $E_{\text {truss }}=32 \pm 4 \mathrm{GPa}$.

\subsection{Hybrid Core Testing}

\subsubsection{Out of plane compression}

The through thickness compressive response of the panels was measured with a screw-driven universal testing machine (Model 4208 Instron Corporation, Canton, MA) with a $300 \mathrm{kN}$ load cell in accordance with ASTM C-365, the Standard Test Method for Flatwise Compressive Properties of Sandwich Cores. Retro-reflective tabs were attached to the top and bottom face sheets, and strain measurements were taken using a laser extensometer. The samples were compressed at a nominal strain rate of $2 \times 10^{-4} \mathrm{~s}^{-1}$ at $23^{\circ}$ C. The elastic modulus for each sample was measured by unloading within the nominally elastic region of the stress-strain curve. 

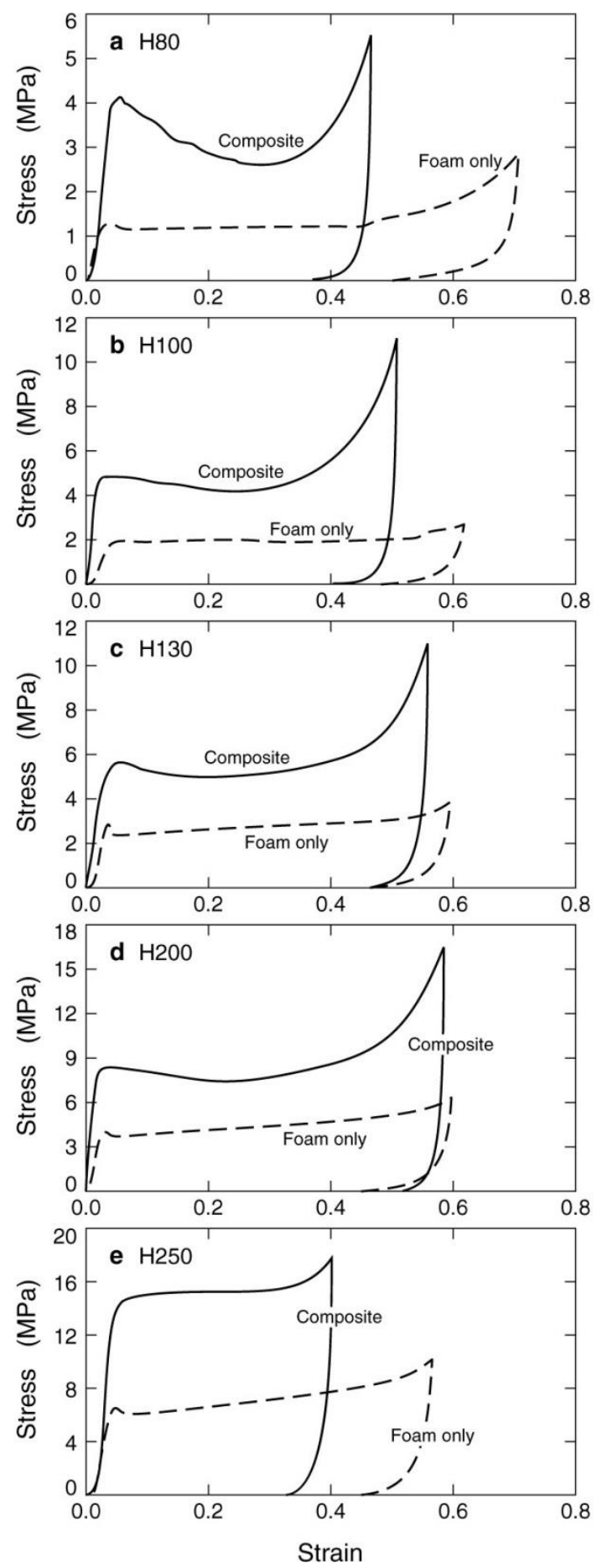

Figure 5.10: Compressive stress-strain responses for the hybrid CFRP pyramidal lattice/foam core panels constructed using Divinycell foams of different strengths (and densities). The response of just the foams is also shown. 

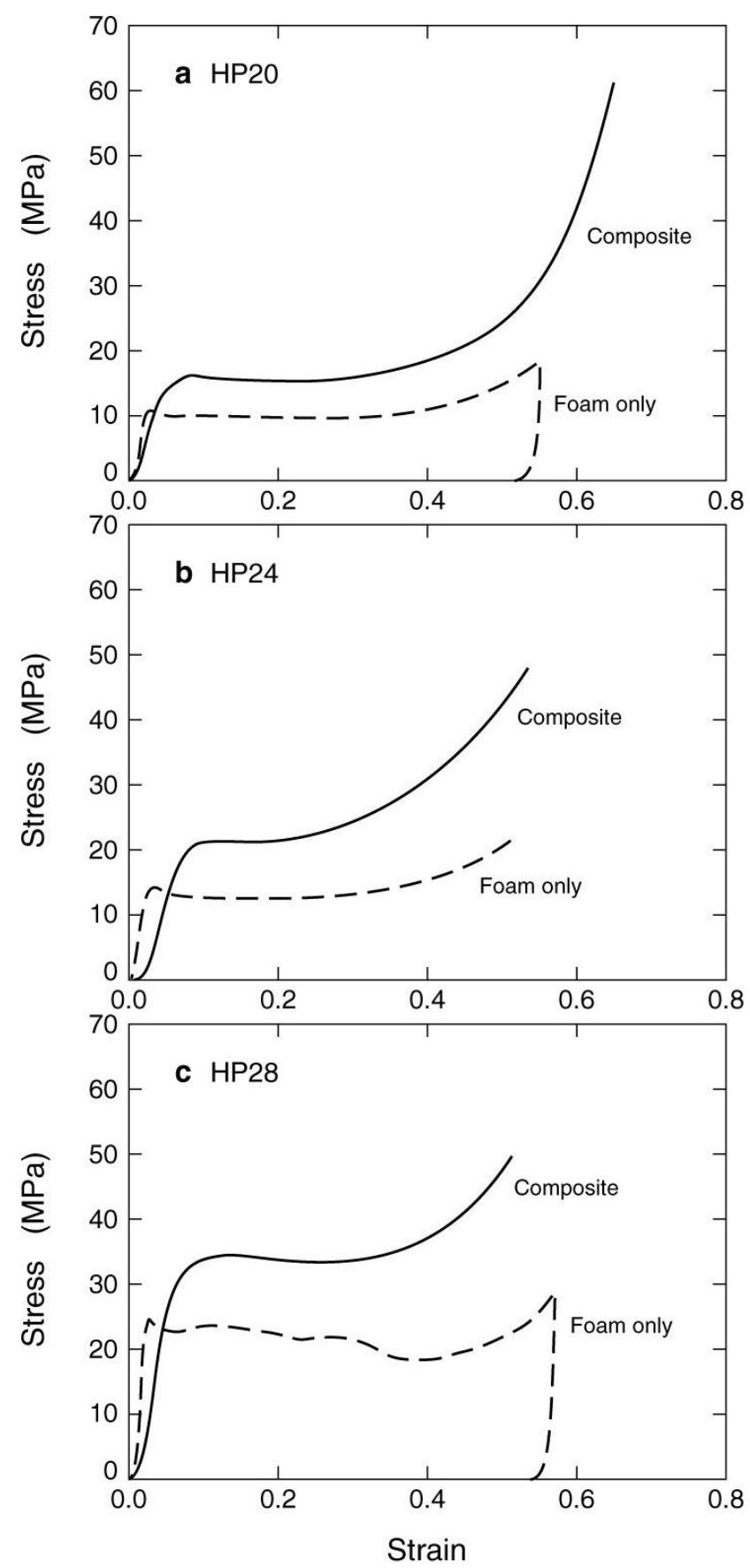

Figure 5.11: Compressive stress-strain responses for the hybrid composite core panels constructed from syntactic foams of different strengths. 
Compressive stress strain responses for the hybrid composite cores constructed with Divinycell and syntactic foams are shown in Figures 5.10 and 5.11, and compared with those of the foams used to fabricate each hybrid core. Two tests were done for each of the core densities, as well as the foams. The average compressive strength (the fracture stress) and modulus for each of the hybrid cores are summarized in Table 2. The stress strain curves for all the samples were initially linear, followed by yielding and a sample dependent drop in strength that was followed by a stress plateau before the stress rose as the core densified. It can be seen that the difference between the strength of the hybrid core and that of the foam, decreased as the foams compressive strength decreased. The reasons for this are investigated in Chapter 6.

Table 5.2: Summary of core properties

\begin{tabular}{|l|c|c|c|c|c|c|c|c|c|}
\hline Foam Type & $\begin{array}{c}\text { None } \\
\text { (Empty) }\end{array}$ & H80 & H100 & H130 & H200 & H250 & HP20 & HP24 & HP28 \\
\hline $\begin{array}{l}\text { Core density } \\
\left(\mathrm{kgm}^{-3}\right)\end{array}$ & 44 & 120 & 141 & 171 & 239 & 288 & 356 & 418 & 482 \\
\hline $\begin{array}{l}\text { Compressive } \\
\text { strength (MPa) }\end{array}$ & 1 & 4.2 & 5.2 & 6 & 8.1 & 12.5 & 16 & 22.5 & 34.5 \\
\hline $\begin{array}{l}\text { Compressive } \\
\text { modulus(MPa) }\end{array}$ & 50.7 & 172 & 251.5 & 315 & 402.8 & 477.1 & 485 & 564 & 755 \\
\hline $\begin{array}{l}\text { Shear strength } \\
\text { (MPa) }\end{array}$ & 0.5 & 1.7 & 3.2 & - & - & - & - & - & - \\
\hline $\begin{array}{l}\text { Shear modulus } \\
(\mathrm{MPa})\end{array}$ & 20.7 & 77 & 123 & - & - & - & - & - & - \\
\hline $\begin{array}{l}\text { Ellipticity }\left(d_{1} / d_{2}\right) \\
(\mathrm{mm} / \mathrm{mm})\end{array}$ & $\begin{array}{l}2.1 / \\
5.3\end{array}$ & $\begin{array}{l}2.8 / \\
5.1\end{array}$ & $\begin{array}{l}2.9 / \\
5.1 *\end{array}$ & $\begin{array}{l}3.0 / \\
5.0^{*}\end{array}$ & $\begin{array}{l}3.2 / \\
4.9\end{array}$ & $\begin{array}{l}3.9 / \\
4.5\end{array}$ & $\begin{array}{l}3.6 / \\
4.5\end{array}$ & $\begin{array}{c}4.1 / \\
4.5^{*}\end{array}$ & $\begin{array}{l}4.3 / \\
4.5\end{array}$ \\
\hline
\end{tabular}

* Indicates interpolated data 
X-ray computed tomography was performed on compressed hybrid core specimens in order to identify the failure mechanisms controlling the cores peak strength. Scans were first performed on unstrained specimens and then repeated after the specimens had been loaded to strain levels of 10,20, and 40\%. Three dimensional reconstructions were performed using volume graphics software (VG Studio 2.0, Volume Graphics GmbH, Heidelberg, Germany). The reconstructed images at the various strain levels for a hybrid CFRP truss/H250 foam core are shown in Figure 5.12. The shape and position of the trusses, as well as the resin sheet can be clearly seen in the unstrained sample, Figure 5.12(a). The foam has been digitally filtered from the image to allow an unimpeded view of the trusses and resin sheet. From the stress-strain curve, it can be seen that the panel yielded at a strain of about 5\%. The XCT reconstruction of a panel compressed to $10 \%$ strain is shown in Figure 12(b). It can be seen that the panel yield corresponded to fracture of the trusses near the nodes with the crack plane at an acute angle with respect to the truss axis. This was similar to the mode observed for a single compressed CFRP braided strut. The resin sheets began to buckle at a strain of $20 \%$, Figure 5.12(c). The onset of densification (strain of 40\%), Figure 5.12(d), coincided with contact of the buckled resin sheets with the face sheets.

The compressive stress versus strain response of an empty lattice made using a low strength (but easily removed) polyurethane foam is shown in Figure 5.13a. The trusses within the empty lattice fracture at the same location as those within the hybrid core. In compression, the empty lattice yielded at a stress of 1MPa. The measured modulus was 50.7 MPa. Further straining led to a rapid, progressive drop in strength. 
a Unstrained sample

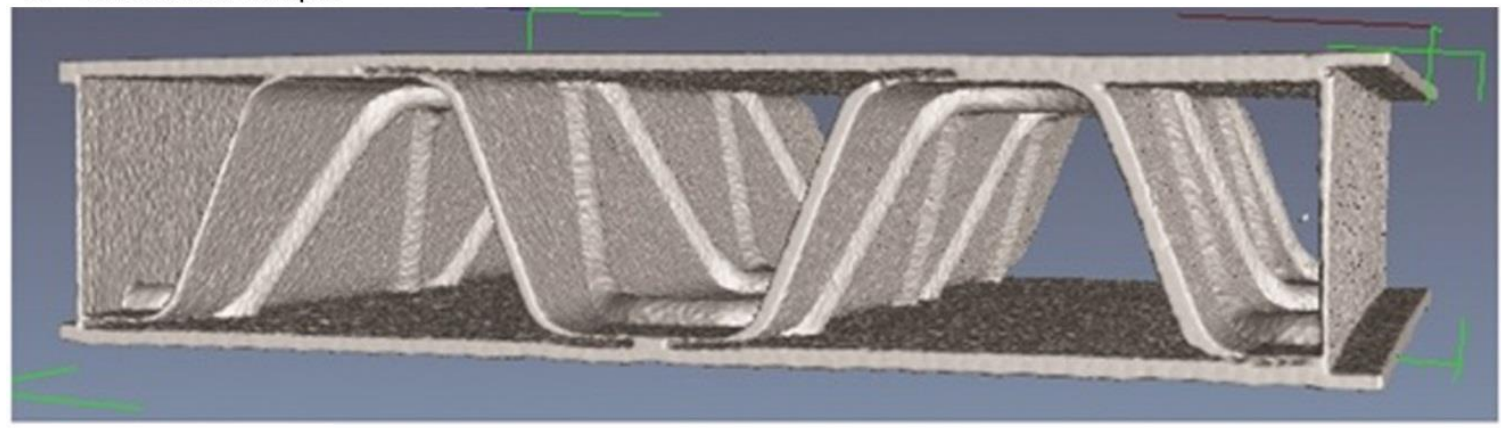

b $10 \%$ strain

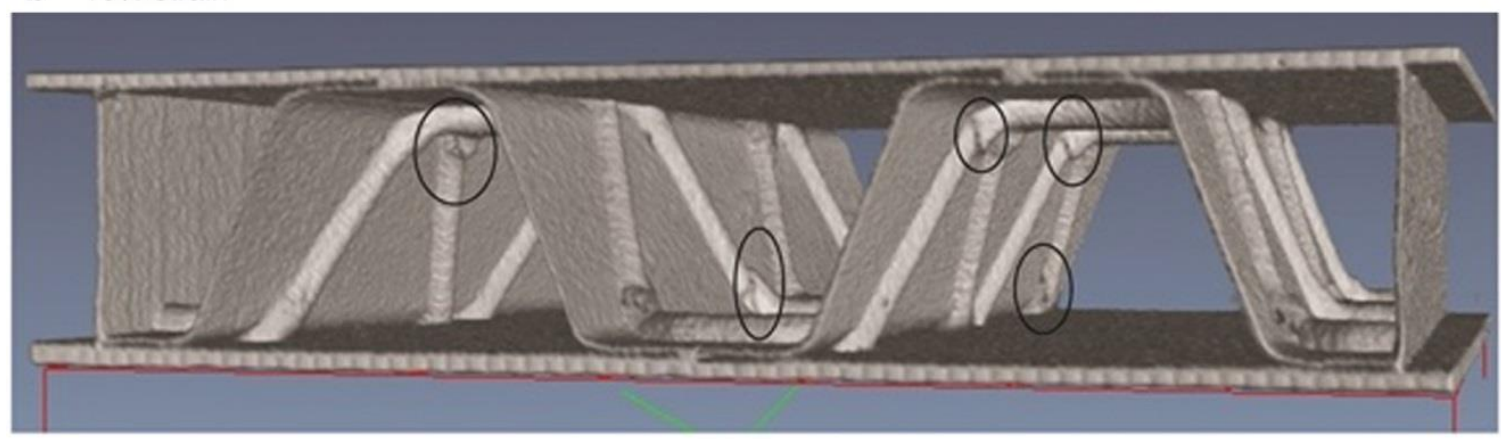

c $20 \%$ strain

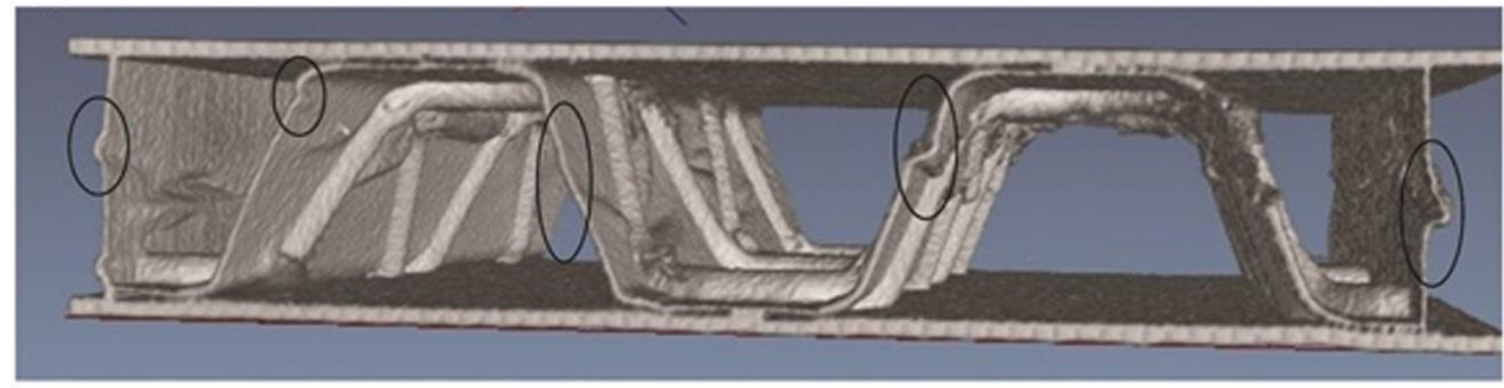

d $40 \%$ strain

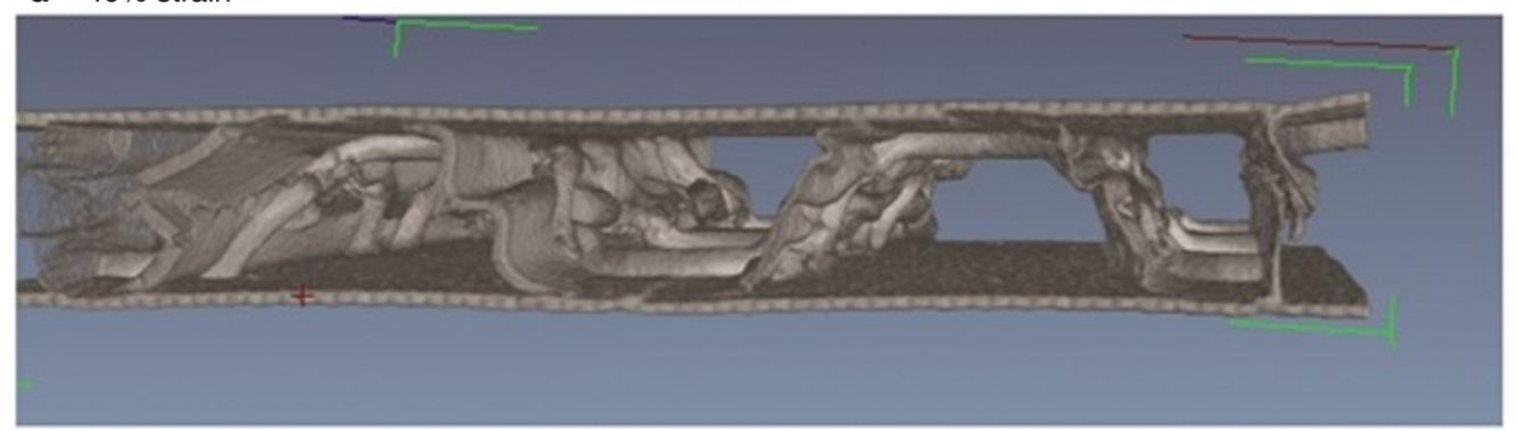

Figure 5.12: Reconstructed X-CT images of a hybrid composite panel with $\mathrm{H} 250$ foam core; (a) as fabricated and at various levels of strain (b-d). The trusses fail by microbuckling near the nodes while the resin sheets fail by buckling. 

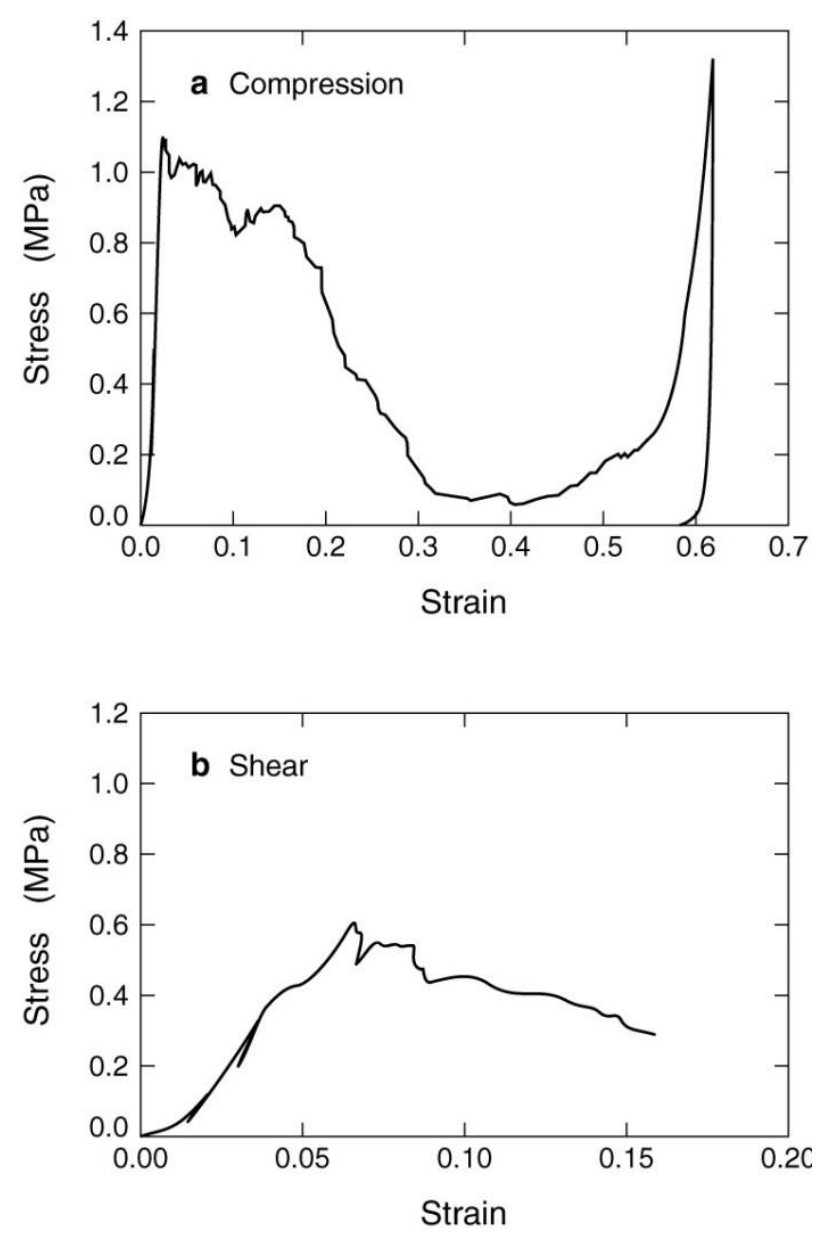

Figure 5.13: The mechanical response of the empty CFRP lattice in (a) compression and (b) shear.

\subsubsection{Role of resin sheet on panel strength}

During the infusion process for the panel, a thin layer of resin formed between the foam molds. The thickness of this layer typically varied between 0.1 and $1 \mathrm{~mm}$. Given the moderately high strength of the epoxy used, it is necessary to determine the contribution of this resin sheet to the strength of the hybrid core. In order to do this, panels were assembled using polymer foam molds as before, but contained no grooves or 
braided trusses. These panels were infused in the same manner and the compressive stress-strain responses of these "no-CFRP truss" cores were measured for the H80, H200, and HP20 foams, and compared to the solid foam cores, Figure 5.14. It can be seen that the resin sheets add about $1 \mathrm{MPa}$ to the compressive strength of the foam filled panel without resin sheets, and was independent of the density of the foam. It is therefore reasonable to conclude that about $1 \mathrm{MPa}$ of the strength difference between the hybrid and foam only cores described in section 4.2 can be attributed to the presence of the resin sheets. It is noted that the densification strain decreases for the panels containing the resin sheets, and this might be attributable to the higher specific strength of the resin. The densification mechanism is discussed in detail in Chapter 9.

\subsubsection{In plane shear}

The in-plane shear response of the panels was measured with the same screw driven universal testing machine using a compression shear plate setup previously described in Chapter 3. The shear testing was performed in accordance with ASTM C273, which dictated that the sample length was twelve times its thickness and the width was two times the thickness. These specifications were satisfied by preparing samples that were two unit cells wide and six unit cells in length. The samples were attached to the shear plates using Redux 319 epoxy adhesive (Hexcel Corporation, Stamford, CT), as well as a set of screws that penetrated the face sheets. The shear plates also had edge stops to provide additional sliding restraint. The testing was performed at $23^{\circ} \mathrm{C}$ and at an angle of $\eta=0$ (in the x-direction) as defined in Figure 5.7. This orientation places two trusses of each unit cell in axial compression, and two in axial tension. Tests in shear 
were successful for the empty lattice (polyurethane foam removed), H80 foam, and the H100 foam hybrids. Efforts to test panels with denser foam cores resulted in failure of the adhesive bond between the composite and the shear plates, and were therefore abandoned.
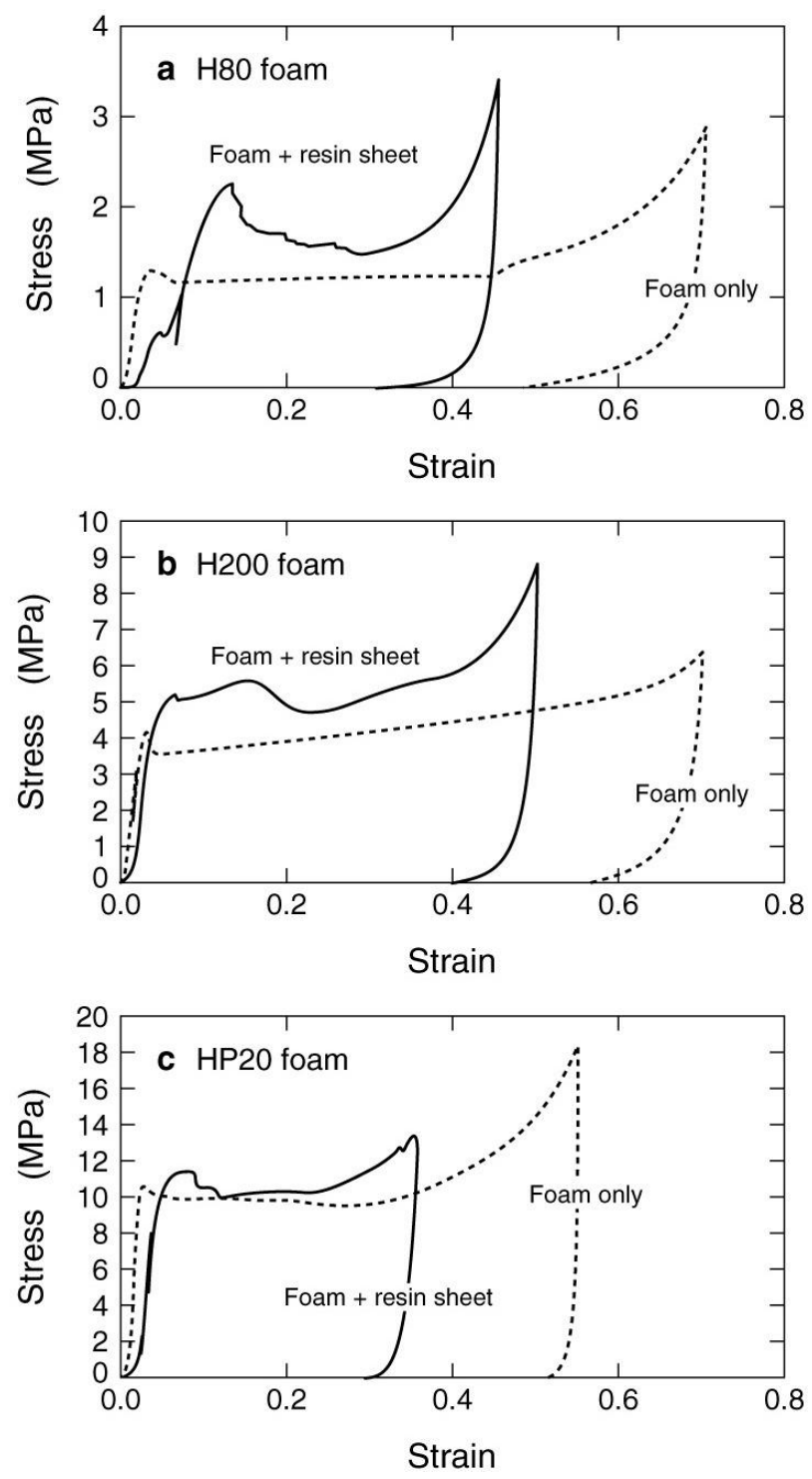

Figure 5.14: Core compressive stress - strain response in the absence of a truss core for 3 foam densities. 
The shear stress-shear strain curves for the successfully tested hybrid core panels are shown in Figure 5.15, along with the curves for the corresponding foams. In shear, the empty lattice failed at a peak strength of $0.5 \mathrm{MPa}$, and had a measured shear modulus of 20.7 MPa, Figure 5.13(b). Failure first occurred within the trusses loaded in tension; a consequence of the larger tensile stress in the tensile loaded struts of a pyramidal lattice with inclination angle $\omega=54^{\circ}$. Figure 5.15 shows a summary of the measured compressive and shear strengths of the composite cores, as well as the foams.
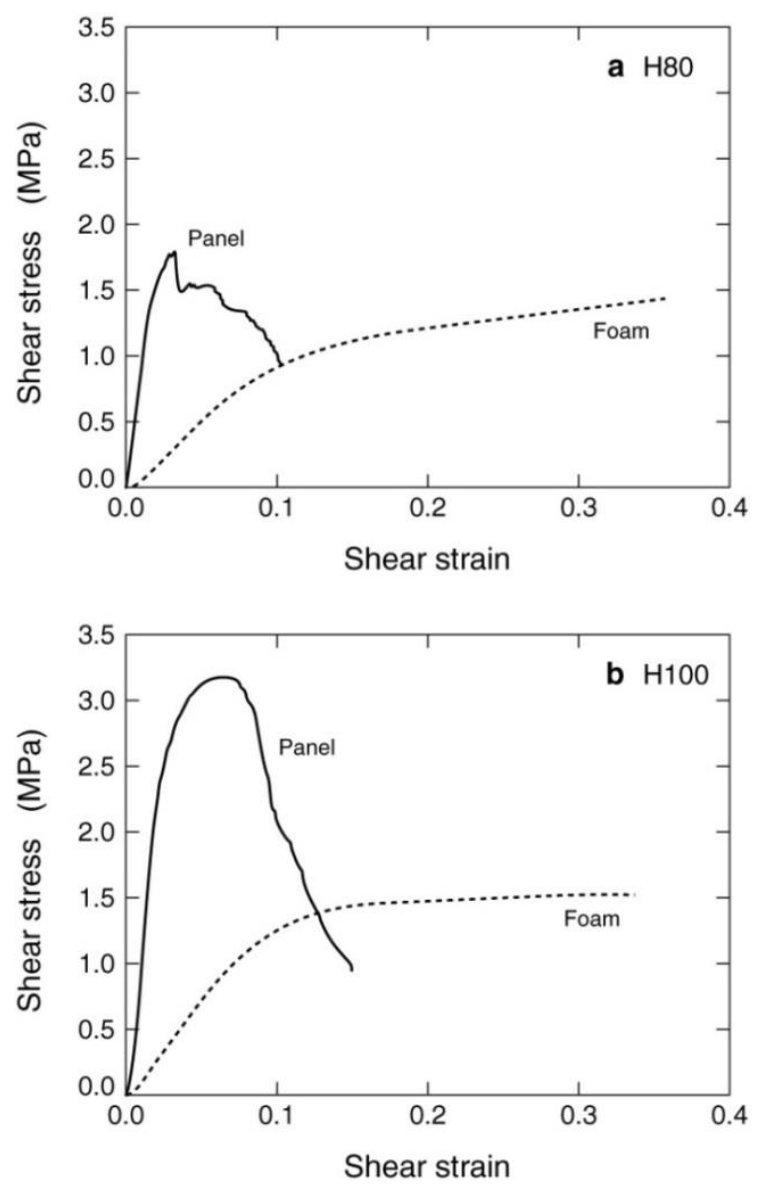

Figure 5.15: Shear stress versus shear strain curves for the hybrid composite core sandwich panels made using (a) H80 and (b) H100 foam. The stress strain curves for the foams used are also shown. 


\section{Chapter 6}

\section{Model of the braided CFRP/Foam Hybrid Core}

To interpret the experiments in Chapter 5, analytical expressions are derived for the compressive and shear moduli and strengths of both the empty CFRP lattice and foam filled hybrid cores. We utilize a local Cartesian coordinate system, with the axes $x, y$, and $z$ in the length, height, and width directions of the unit cell, Figure 5.7.

\subsection{Core Compression}

\subsubsection{Compressive modulus}

When an out of plane compressive force is applied to the top of a rigidly supported empty lattice unit cell with oval cross section trusses, Figure 6.1, axial, $F_{A}$, and shear, $F_{S}$ forces are created in each of the trusses. Using the approach developed by Finnegan et $\mathrm{al}^{4}$, these forces can be related to geometric parameters of the unit cell and the material used to make it. The axial force, $F_{A}$ is dependent on the cross-sectional area of the truss, and the shear force, $F_{S}$, is also dependent on the second moment of the crosssectional area of the beam. These truss supported forces, using elemental beam theory, are given by the bending moment and deflection, as shown below:

$$
F_{A}=\frac{E_{\text {truss }} \pi r_{1} r_{2} \delta \sin \omega}{l}
$$


and

$$
F_{s}=\frac{E_{\text {truss }} \pi r_{1}^{3} r_{2} \delta \cos \omega}{l^{3}}
$$

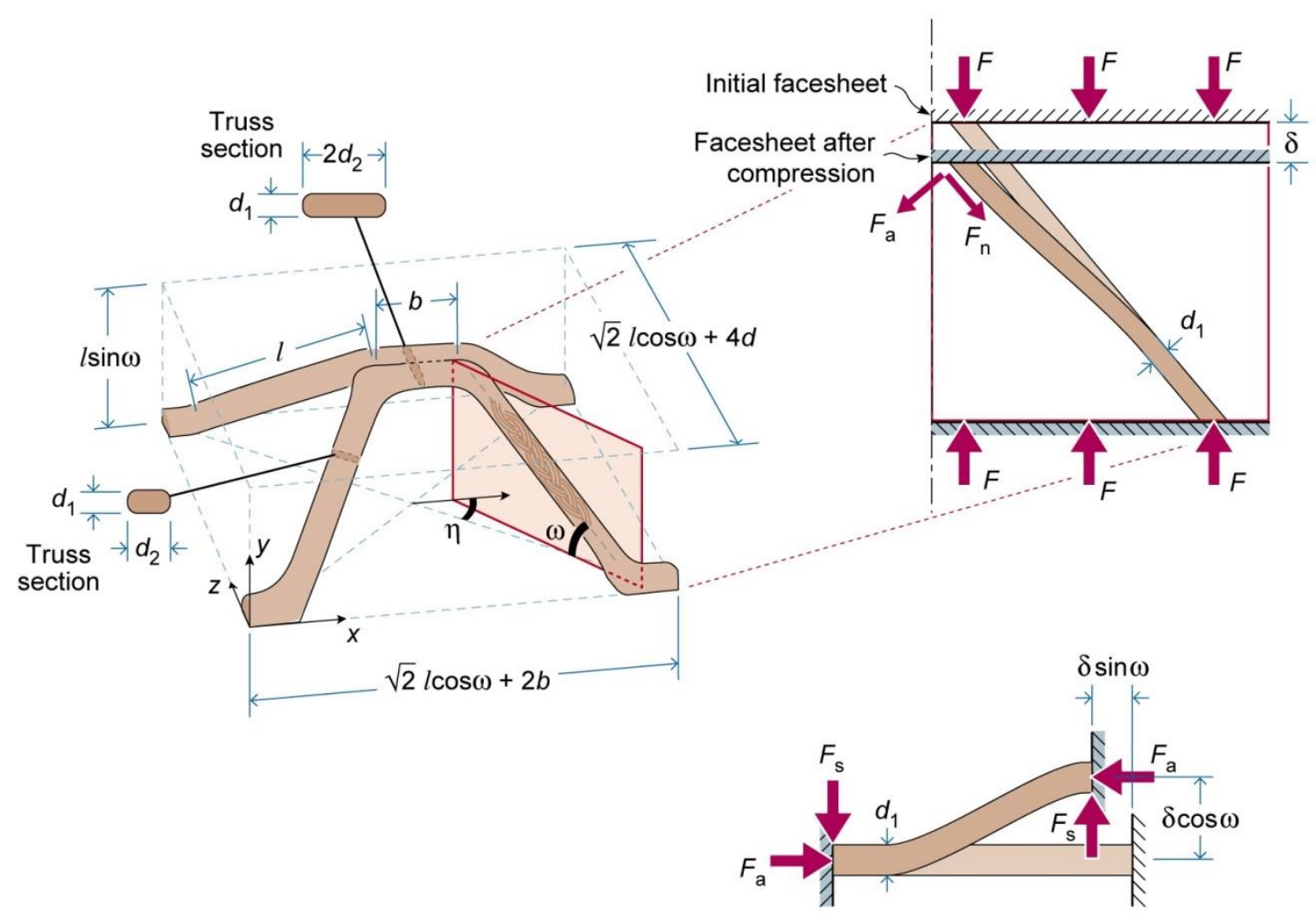

Figure 6.1: Schematic and free body diagram showing deflection of the struts within the core upon application of a compressive load.

where $r_{1}=d_{1} / 2$ and $r_{2}=d_{2} / 2$ and $\delta$ is the unit cell displacement in the y direction (recall that the trusses are bending about the $d_{2}$ axis). In the limit of a circular cross section truss, these expressions reduce to:

$$
F_{A}=\frac{E_{\text {truss }} \pi r^{2} \delta \sin \omega}{l}
$$


and

$$
F_{s}=\frac{E_{\text {truss }} \pi r^{4} \delta \cos \omega}{l^{3}}
$$

The total force acting on the end of the truss, $F$ is the sum of the axial and shears forces:

$$
F=F_{A} \sin \omega+F_{S} \cos \omega=\frac{E_{\text {truss }} \pi r_{1} r_{2} \delta}{l}\left[\sin ^{2} \omega+\left(\frac{r_{1}^{2}}{l^{2}}\right) \cos ^{2} \omega\right]
$$

Since there are four struts within a CFRP pyramidal unit cell, each supporting an applied force $\mathrm{F}$ (Equation 6.3), and the base area of the unit cell is known, the stress supported by an empty lattice is given by:

$$
\sigma=\frac{8 F}{\left(\sqrt{2} l \cos \omega+4 d_{2}\right)(\sqrt{2} l \cos \omega+2 b)}
$$

The definition of compressive strain for the unit cell is given by:

$$
\varepsilon \equiv \frac{\delta}{l \sin \omega}
$$


Since the compressive modulus of the pyramidal lattice, $E_{\text {lattice }}=\sigma / \varepsilon$, it follows from Equations $6.4 \mathrm{a}$ and $6.4 \mathrm{~b}$ that:

$$
\frac{E_{\text {lattice }}}{E_{\text {truss }}}=\frac{8 \pi r_{1} r_{2} \sin \omega\left[\sin ^{2} \omega+\left(\frac{r_{1}^{2}}{l^{2}}\right) \cos ^{2} \omega\right]}{\left(\sqrt{2} l \cos \omega+4 d_{2}\right)(\sqrt{2} l \cos \omega+2 b)}
$$

where $E_{\text {truss }}$ is the compressive elastic modulus of the braided CFRP truss, as reported in Chapter 5.

The contribution of the corrugated resin sheet to the modulus of the core is dependent on the elastic modulus of the resin, $E_{\text {resin }}$, the volume fraction of the resin corrugation, $v_{f r}$, and the angle of inclination of the resin sheets, $\theta$. Deshpande, et al ${ }^{19}$ have shown that this contribution from the corrugated structure, following a resolution of forces, can be expressed as:

$$
E_{c o r r}=v_{f r} E_{r e s i n} \sin ^{4} \theta
$$

Assuming the strains in the foam, resin sheet and trusses of the compressed hybrid core to be identical, the compressive modulus of the foam filled hybrid, $E_{c}$, can be determined using a rule of mixtures:

$$
E_{c}=E_{\text {lattice }}+v_{f f} E_{\text {foam }}+v_{f r} E_{\text {resin }} \sin ^{4} \theta
$$

where $v_{f r}$ is the volume fraction of the resin $(0.025)$, and $E_{\text {resin }}$ the elastic modulus of the resin and $v_{f f}$ is the volume fraction of the foam $\left(1-\bar{\rho}-v_{f r}\right), \theta$ is the angle of inclination of 
the resin sheet with respect to the X-Z plane, and $E_{\text {foam }}$ is the elastic modulus of the foam. Figure 6.2(a) shows that the predicted and measured moduli, while Figure 6.2(b) shows the contribution of each of the components within the hybrid core system (truss, resin sheet and foam) to the total predicted modulus. It can be seen that the model overpredicts the modulus. This might be attributable to the fact that even a small amount of truss waviness introduced during panel manufacture could lead to large knockdowns in the measured stiffness, an effect documented by Queheillalt, et $\mathrm{al}^{59}$.

\subsubsection{Compressive Strength}

For low aspect ratio trusses, axial loading of braided CFRP strut in the axial direction results in either Euler elastic or plastic microbuckling. For microbuckling failure, Argon ${ }^{49}$ has argued that the compressive strength, $\sigma_{\max }$ of an axially loaded composite made up of fibers within a plastic matrix with shear yield strength $\tau_{y}$ is given by:

$$
\sigma_{\max }=\frac{\tau_{y}}{\varphi}
$$

where $\varphi$ is the axial misalignment angle of the fibers. In a pyramidal lattice loaded in through thickness compression the struts are loaded in both axial compression and inplane shear ${ }^{4}$. An in-plane shear stress $\tau^{\infty}$ is introduced due to inclination of the struts. When this in-plane shear stress is superimposed on the axial compressive stress, Fleck and Budiansky ${ }^{50}$ have shown that the critical microbuckling stress $\sigma_{c}$ can be approximated by: 


$$
\sigma_{c}=\frac{\tau_{y}-\tau^{\infty}}{\varphi}
$$

where $\tau^{\infty}<\tau_{\mathrm{y}}$, is the in-plane shear stress which reduces the critical axial stress required to cause microbuckling.
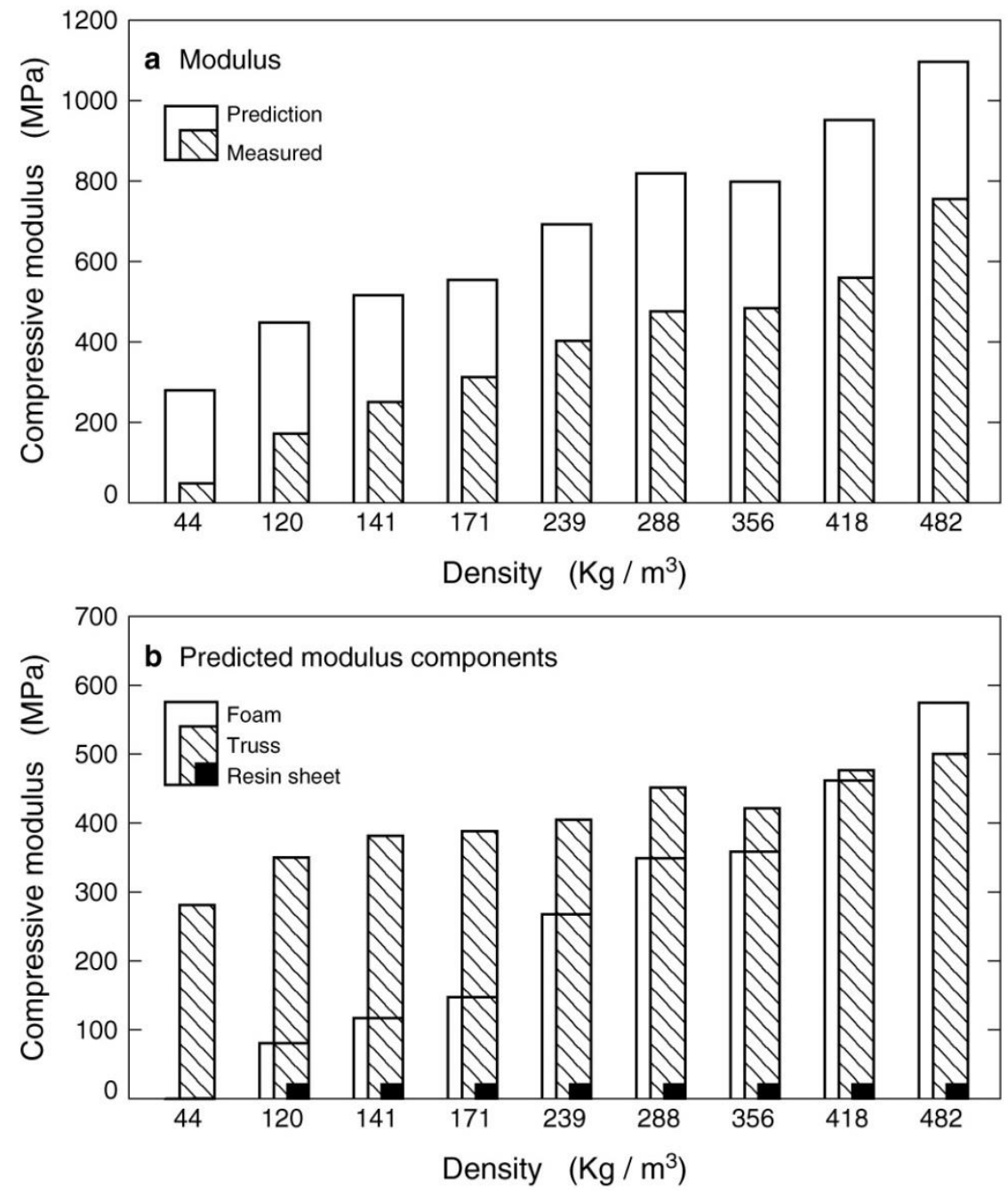

Figure 6.2: Barcharts showing: (a) the measured compressive modulus compared to the predicted modulus for select foam densities, and (b) the individual modulus components combined for the cumulative prediction $\left(\mathrm{E}_{\mathrm{core}}\right)$ for each of the specified core densities. 
Since $\tau^{\infty}$ and $\sigma_{\mathrm{c}}$ can be obtained from Equations 6.2a and 6.2b divided by the truss cross sectional area, the ratio of $\tau^{\infty} / \sigma_{\mathrm{c}}=(r / l)^{2} \cot \omega$. Upon substitution for $\tau^{\infty}$ in Equation 6.8 and rearranging, we find that the critical buckling strength under combined axial compression and in-plane shear can be written as:

$$
\sigma_{c}=\frac{\tau_{y}}{\varphi+\left(\frac{r_{1}}{l}\right)^{2} \cot \omega}=\frac{\sigma_{\max }}{\left[1+\left(\frac{r_{1}}{l}\right)^{2} \frac{\cot \omega}{\varphi}\right]}
$$

From Equation 6.3, the critical buckling force supported by a single inclined strut is:

$$
F=\sigma_{c} \pi r_{1} r_{2} \sin \omega\left[\sin ^{2} \omega+\left(\frac{r_{1}}{l}\right)^{2} \cos ^{2} \omega\right]
$$

Since there are four trusses per unit cell, and the area of the unit cell is known, the microbuckling strength of the pyramidal lattice can be written as:

$$
\sigma_{p} \equiv \frac{8 \sigma_{c} \pi r_{1} r_{2} \sin \omega}{\left(\sqrt{2} l \cos \omega+4 d_{2}\right)(\sqrt{2} l \cos \omega+2 b)}\left[\sin ^{2} \omega+\left(\frac{r_{1}}{l}\right)^{2} \cos ^{2} \omega\right]
$$

From Equation 6.9, it can be seen that this expression for the peak strength of the core can be rewritten as: 


$$
\frac{\sigma_{p}}{\sigma_{\max }}=\frac{8 \pi r_{1} r_{2} \sin \omega}{\left(\sqrt{2} l \cos \omega+4 d_{2}\right)(\sqrt{2} l \cos \omega+2 b)\left[1+\left(\frac{r_{1}}{l}\right)^{2} \frac{\cot \omega}{\varphi}\right]}\left[\sin ^{2} \omega+\left(\frac{r_{1}}{l}\right)^{2} \cos ^{2} \omega\right]
$$

In the special case of a cylindrical truss; $r_{1}=r_{2}=r$, this expression reduces to:

$$
\frac{\sigma_{p}}{\sigma_{\max }}=\frac{8 \pi r^{2} \sin \omega}{(\sqrt{2} l \cos \omega+4 d)(\sqrt{2} l \cos \omega+2 b)\left[1+\left(\frac{r}{l}\right)^{2} \frac{\cot \omega}{\varphi}\right]}\left[\sin ^{2} \omega+\left(\frac{r}{l}\right)^{2} \cos ^{2} \omega\right]
$$

Equations $6.12 \mathrm{a}$ and $\mathrm{b}$ are evaluated using the compressive strength of the truss $\sigma_{\max }$ determined in Chapter 5, as well as unit cell parameters $\omega, l, b$, and $d$ also described in Chapter 5. In addition $r_{1}$ and $r_{2}$ were determined from the $d_{1}$ and $d_{2}$ values listed in Table 5.2. The fiber misalignment angle is taken to be the braid angle $11^{\circ}$.

The microbuckling mechanism competes with an Euler (elastic) buckling mode. The critical Euler buckling load, $P_{E}$ is given by:

$$
P_{E}=\frac{\pi^{2} E I}{L^{2}}
$$

where $E$ is the Young's modulus of the beam, $I$ is the second area moment, and $L$ is the length of the beam. For an elliptical cross-section truss, the second area moment, $I \equiv \pi r_{1}^{3} r_{2} / 4$, where $r_{1}$ and $r_{2}$ are the short and long radii of the elliptical. The critical buckling stress for an elliptical truss is given by: 


$$
\sigma_{e l}=\frac{\pi^{2} r_{1}^{3} r_{2} E_{\text {truss }}}{4 l^{2}}
$$

For a cylindrical truss, $I \equiv \pi r^{4} / 4$, and the elastic buckling stress reduces to:

$$
\sigma_{e l}=\frac{\pi^{2} r^{4} E_{\text {truss }}}{4 l^{2}}
$$

The peak elastic buckling strength of the core can then be found by substituting $\sigma_{\mathrm{el}}$ for $\sigma_{\max }$ in Equation 6.12.

The predicted microbuckling and elastic buckling strengths of an empty CFRP pyramidal lattice are plotted as a function of the $d_{1} / d_{2}$ ratio for $d_{2}=4.5$ (core with strongest foam) and 5.3mm (weakest polyurethane foam) in Figure 6.3. It can be seen that as the truss became more oval (decreased $d_{1} / d_{2}$ ), the elastic buckling stress becomes lower than the microbuckling stress and the strength of lattices made using the polyurethane, H80 and H 200 foams is predicted to be governed by elastic buckling. However, as $d_{1} / d_{2}$ approached unity, the plastic microbuckling stress became lower than the elastic buckling stress, and failure is predicted to occur by microbuckling for the lattices fabricated with syntactic foams (HP series of foams). The deduced lattice contribution $^{\mathrm{ii}}$ to the compressive strength is overlaid on the predictions in Figure 6.3. The

\footnotetext{
ii Obtained by subtracting the strength of the foam and resin sheet from the measured strength of the hybrid
} core. 
trends with $\mathrm{d}_{1} / \mathrm{d}_{2}$ ratio are similar to that predicted, but the strength levels are lower, which is consistent with the imperfection sensitivity of the failure modes.

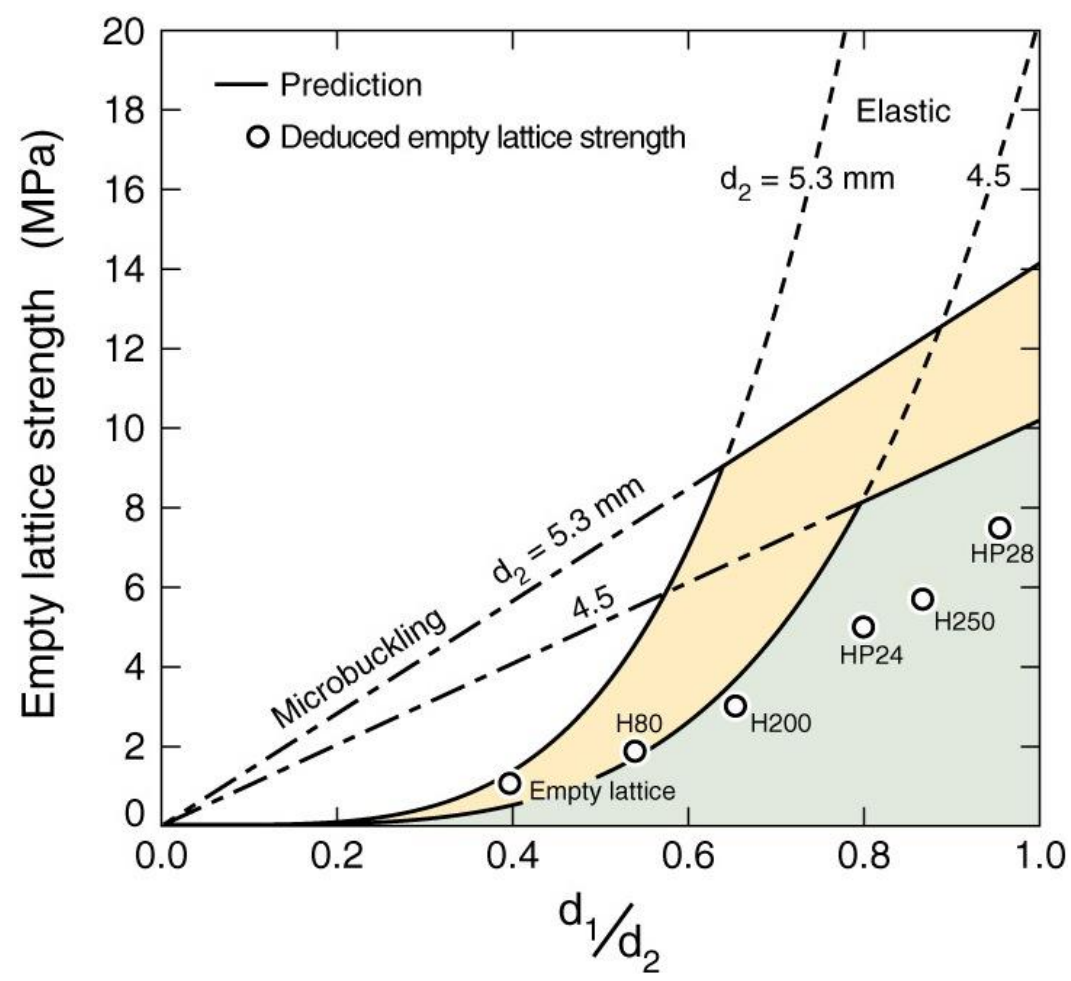

Figure 6.3: The micromechanical predictions for both the elastic and microbuckling strengths plotted as a function of the ellipticity ratio $d_{1} / d_{2}$. The compressive strength contribution of the CRFP lattice deduced by subtracting the contributions of the foam and resin sheets from the measured strength are also shown for the different foams (open circles).

The contribution of the corrugated resin sheet to the compressive strength of the core is dependent on the compressive strength of the resin, $\sigma_{r}$, the volume fraction of the resin corrugation, $v_{f r}$, and the angle of inclination of the resin sheets, $\theta$. Deshpande, et al ${ }^{19}$ 
have shown that this contribution from the corrugated structure, following a resolution of forces, can be expressed as:

$$
\sigma_{\text {corr }}=v_{f r} \sigma_{r} \sin ^{2} \theta
$$

The compressive strength of the foam filled hybrid core can be predicted by assuming iso-strain conditions in the lattice, foam and polymer corrugation. The rule of mixtures compressive strength of the hybrid core $\sigma_{h c}$ can then be written as:

$$
\sigma_{h c}=v_{f f} \sigma_{f}+v_{f r} \sigma_{r} \sin ^{2} \theta+\sigma_{p}
$$

where $v_{f f}$ is the volume fraction of the foam within the composite core unit cell, and $v_{f r}$ is the volume fraction of the resin sheets, $\theta$ is the angle of inclination of the resin sheets with respect to the direction of compression, and $\sigma_{f}$ and $\sigma_{r}$ are the compressive strengths of the foam and resin, respectively. Figure 6.4 compares the measured compressive strength of the hybrid composite core and the micromechanical predictions for the three contributions to the predicted strength (Equation 6.14). The upper bound micromechanical predictions are in good agreement with the measurements. 

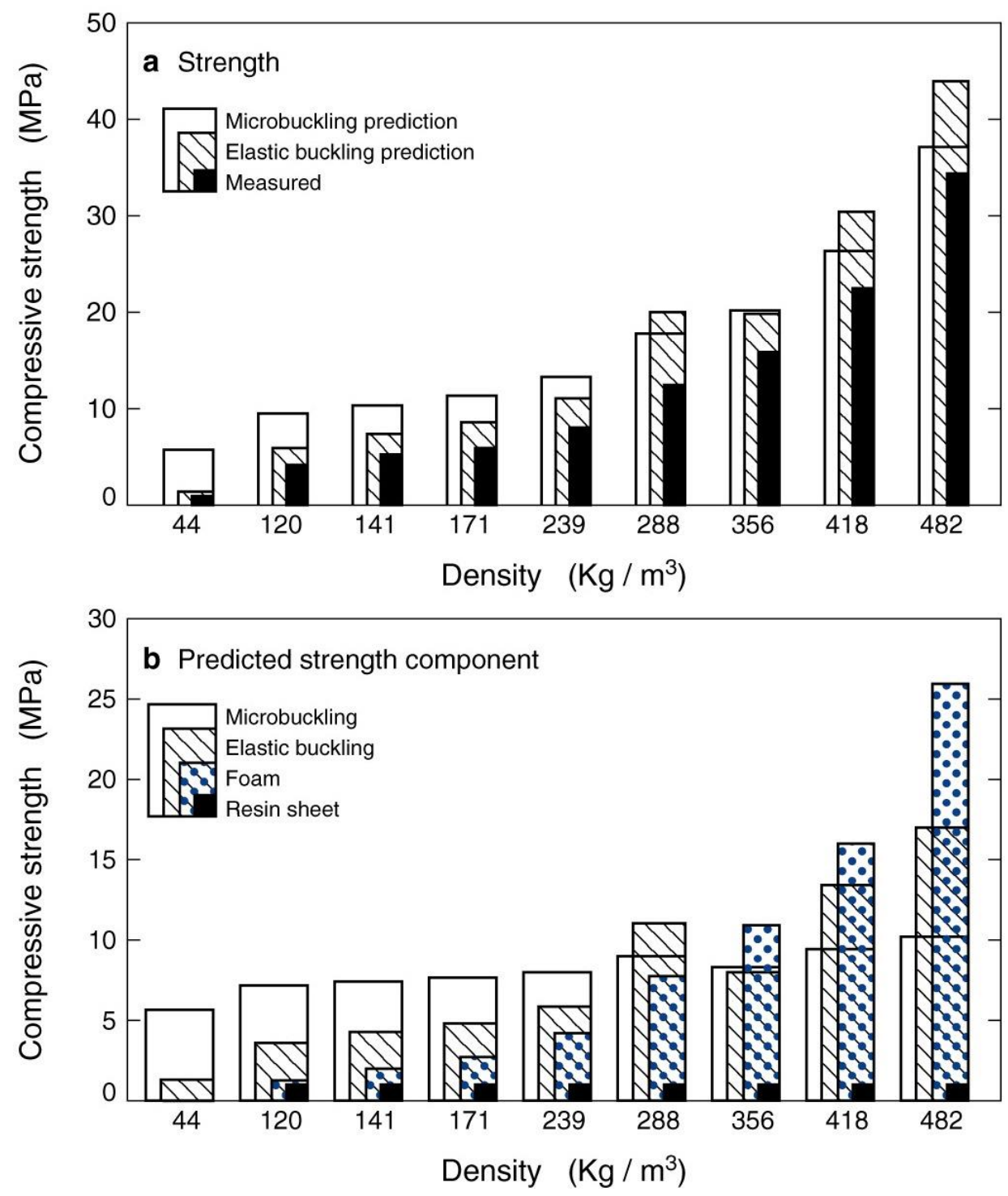

Figure 6.4: Barcharts showing (a) the measured core compressive strength compared to the micromechanical predictions, and (b) the individual components of the compressive strength prediction. 


\subsection{Core Shear}

\subsubsection{Shear Modulus}

Examination of the unit cell in Figure 6.1, shows that an applied shear force along the $\mathrm{x}$-axis at a shearing angle $\alpha=0$, would result in a deflection $\delta$ of the top face of the unit cell and a tensile force on two of the trusses and a compressive force on the remaining trusses. Following the approach outlined in Chapter 4, the unit cell deflection can be resolved into axial and shear displacements $\delta_{a}$ and $\delta_{s}$ of the trusses given by:

$$
\begin{gathered}
\delta_{a}=\delta \cos \omega \\
\delta_{s}=\delta \sin \omega
\end{gathered}
$$

Since the direction of the applied shear force is perpendicular to $y-z$ plane of the unit cell, Figure 6.1, the force is symmetrically distributed among the trusses within the unit cell. Each truss would be subjected to an axial and a shear force, which, following the method outlined in Chapter 4 are given by:

$$
F_{A}=\frac{E_{\text {truss }} \pi r_{1} r_{2} \delta_{a} \sin \omega}{l}
$$

and

$$
F_{s}=\frac{E_{\text {truss }} \pi r_{1}^{3} r_{2} \delta_{s} \cos \omega}{l^{3}}
$$


The total applied force applied to each truss, $F$ is given by:

$$
F=F_{A} \cos \omega+F_{S} \sin \omega=\frac{E_{\text {truss }} \pi r_{1} r_{2} \delta}{l}\left[\cos ^{2} \omega+\left(\frac{r_{1}}{l}\right)^{2} \sin ^{2} \omega\right]
$$

The shear stress, $\tau$ supported by the four trusses is then:

$$
\tau \equiv \frac{4 F}{\left(\sqrt{2} l \cos \omega+4 d_{2}\right)(\sqrt{2} l \cos \omega+2 b)}
$$

The engineering shear strain $\gamma$ is given by:

$$
\gamma \equiv \frac{\delta}{l \sin \omega}
$$

The shear modulus G of the truss-only structure can then be written as:

$$
\frac{G_{\text {lattice }}}{E_{\text {truss }}}=\frac{4 \pi r_{1} r_{2} \sin \omega\left[\cos ^{2} \omega+\left(\frac{r_{1}}{l}\right)^{2} \sin ^{2} \omega\right]}{\left(\sqrt{2} l \cos \omega+4 d_{2}\right)(\sqrt{2} l \cos \omega+2 b)}
$$


The shear modulus of hybrid core should also include contributions from the foam and resin corrugations. Assuming their contribution is given by the rule of mixtures, the hybrid core shear modulus, $G_{c}$ is given by:

$$
G_{c}=\bar{\rho} G_{\text {lattice }}+v_{f f} G_{\text {foam }}+v_{f r} E_{r e s i n} \frac{\sin ^{2} 2 \theta}{4}
$$

where $G_{f o a m}$ is the shear modulus of the foam.

\subsubsection{Shear Strength}

Failure within the specimens tested in shear occurred within the trusses loaded in tension. Thus, the tensile strength of the truss is used to predict the shear strength. It follows from Equations 6.17 and 6.18 that the shear strength, $\tau_{L}$ of the (empty) lattice core can be expressed as:

$$
\frac{\tau_{L}}{\sigma_{\text {tensile }}}=\frac{\pi r_{1} r_{2}\left[\cos ^{2} \omega+\left(\frac{r_{1}}{l}\right)^{2} \sin ^{2} \omega\right]}{\left(\sqrt{2} l \cos \omega+4 d_{2}\right)(\sqrt{2} l \cos \omega+2 b)}
$$

where $\sigma_{\text {tensile }}$ is the tensile strength of the truss, as determined in Chapter 5 . In the special case where the trusses are cylindrical, $r_{1}=r_{2}=r$, the above equation can be rewritten as: 


$$
\frac{\tau_{L}}{\sigma_{\text {tensile }}}=\frac{\pi r^{2}\left[\cos ^{2} \omega+\left(\frac{r}{l}\right)^{2} \sin ^{2} \omega\right]}{(\sqrt{2} l \cos \omega+4 d)(\sqrt{2} l \cos \omega+2 b)}
$$
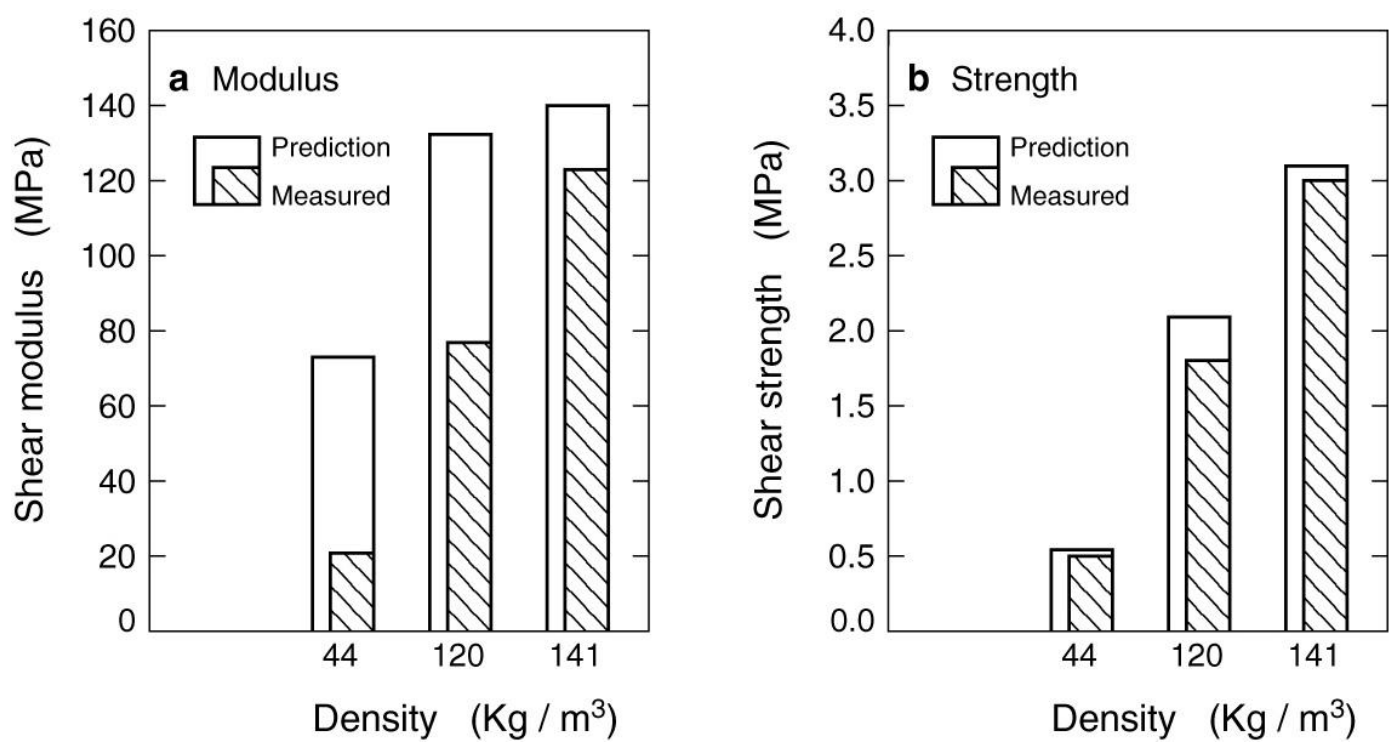

Figure 6.5: (a) The measured shear modulus of the composite cores compared to the micromechanical predictions. (b) The measured shear strength of the composite cores compared to the micromechanical predictions.

The contribution of the corrugated resin sheet to the shear strength of the core is dependent on the strength of the resin, $\sigma_{r}$, the volume fraction of the resin corrugation, $v_{f r}$, and the angle of inclination of the resin sheets, $\theta$. Deshpande, et a ${ }^{19}$ have shown that this contribution from the corrugated structure, following a resolution of forces, can be expressed as: 


$$
\sigma_{c s}=v_{f r} \sigma_{r} \cos ^{2} \theta
$$

The shear strength $\tau_{c}$ of the foam filled hybrid core can be determined by accounting for the individual components using the rule of mixtures:

$$
\tau_{c}=v_{f f} \tau_{f}+v_{f r} \sigma_{t r} \cos ^{2} \theta+\tau_{L}
$$

where $\tau_{\mathrm{f}}$ is the shear strength of the foam, and $\sigma_{\mathrm{tr}}$ is the tensile strength of the cured resin. Figure 6.4 compares the measured shear modulus and strength of the cores to the micromechanical predictions. The modulus is slightly over predicted, as explained earlier. The strength predictions are in pretty reasonable agreement with the measured values.

As the density of the foam decreases, there is a significant knockdown in the measured empty lattice strength of the core, while cores made using stronger foams have higher measured empty lattice strengths. This knockdown is explained by the fact that the weaker foams allow significant deformation of the shape of the trusses during the pressure assisted consolidation process (vacuum and pressure cycle within the autoclave), causing the cross-section of the truss to become more oval, and the $\mathrm{d}_{1} / \mathrm{d}_{2}$ ratio smaller.

\subsection{Energy Absorption}

Compressive and shear moduli and strengths of the hybrid CFRP lattices are plotted against density on modified Ashby charts in Figure 6.5 and compared with the 
foams used here and other CFRP lattices and honeycombs discussed in the introduction. It can be seen that the strength and moduli of the hybrid cellular structures lie between those of the foams and CFRP lattices/honeycombs. The specific strength and modulus of the CFRP lattices with circular cross section struts exceed those of the foams, and so as the mass fraction of the hybrid devoted to the foam increases, the mechanical properties converge to those of the foam and vice versa. A complicating discovery here was that partial compression of the foam inserts during pressure assisted resin transfer caused the originally circular trusses to assume an elliptical cross sectional shape, with the ellipticity increasing as the foams compressive strength was decreased. The reduced resistance of the elliptical shaped CFRP truss to elastic bending led to a significant reduction in the CRFP lattice contribution to the hybrid structures strength as the foam density was decreased. 

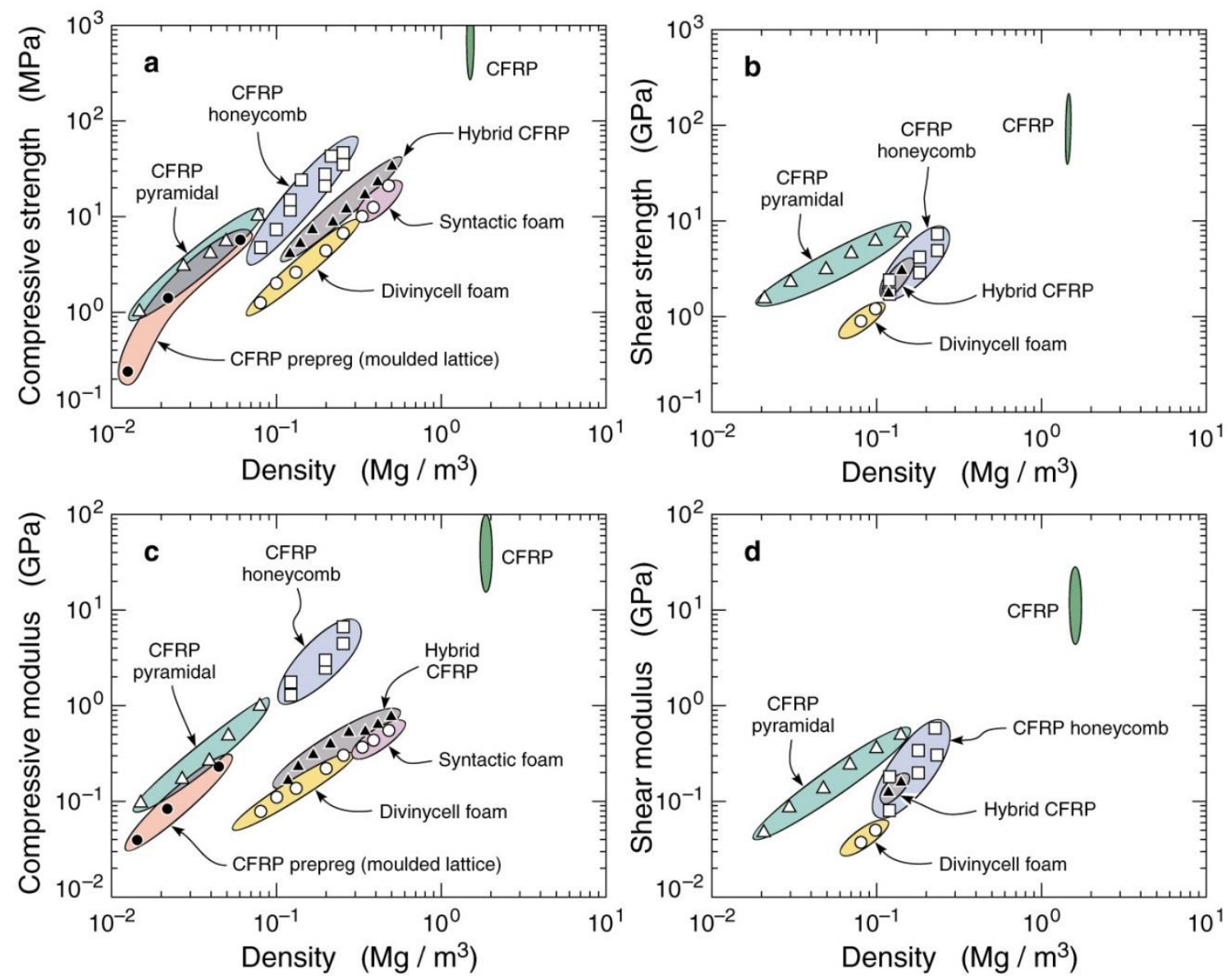

Figure 6.6: Material property chart showing (a) compressive strength, (b) shear strength, (c) compressive modulus and (d) shear modulus of CFRP honeycomb and pyramidal truss structures. The hybrid CFRP lattice/foam results of this study are also shown together with that of the foams.

The compressive stress versus strain responses of the hybrid cores exhibited a foam-like behavior with a well-developed plateau stress that continued to onset of densification, Figures 5.10 and 5.11. They therefore appear well suited for impact energy 
absorption applications. To investigate this further, the energy absorbed per unit volume, $W_{v}$ was determined by integrating the stress strain curve to the onset of densification:

$$
W_{v}=\int_{0}^{\varepsilon_{d}} \sigma d \varepsilon
$$

where $\varepsilon_{d}$ is the densification strain. The densification strain was determined by the point where the stress at the end of the plateau region exceeded the initial peak stress, or in the absence of a sharp initial peak, was defined as the inflection point where the tangent of the plateau region of the stress strain curve intersected the tangent to the densification region $^{60}$. The energy absorbed per unit mass, $W_{m}$, was obtained by dividing $W_{v}$ by the core density.

Figure 6.6(a) shows a plot of the volumetric energy absorption as a function of the density, while Figure 6.6(b) shows a plot of the gravimetric energy absorbed per unit mass, again as a function of the core density. It can be seen that the hybrid core significantly exceeds that absorbed by foams and CFRP lattices and honeycombs. This increase in the energy absorption of the hybrid over the foam is due to the presence of the foams, This suggests that for applications where high crush strength and impact energy absorption are required, these hybrid structures offer an interesting alternative to honeycombs and other core structures. The micromechanical models developed in this chapter provide a simple means for designing structures that meet specified demands for strength and modulus. 

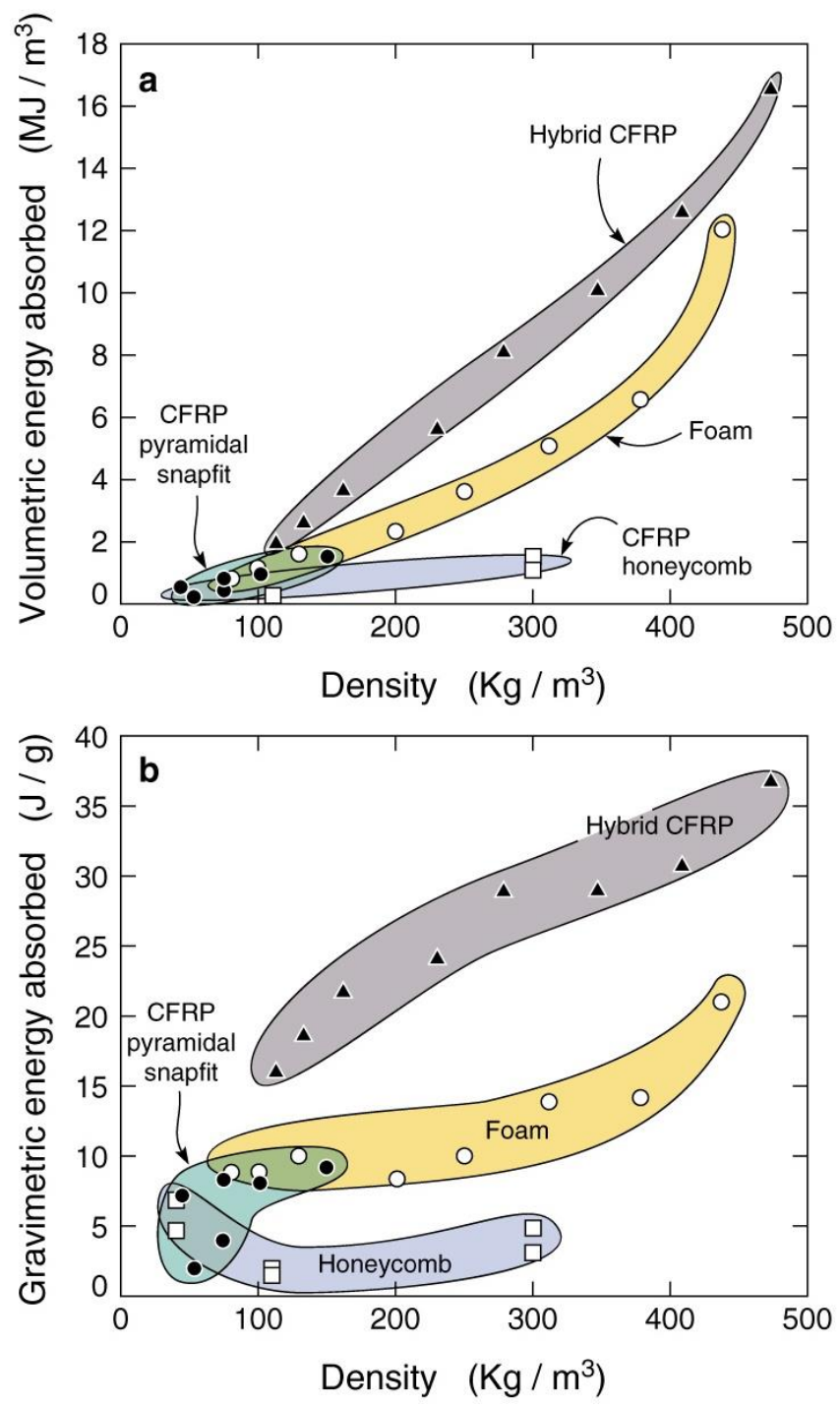

Figure 6.7: (a) The energy absorbed per unit volume and (b) energy absorbed per unit mass both plotted against density for low density cellular materials. The hybrid composites compare favorably with the other materials. (The data on other lattices is aggregated from published work within the IPM group and other groups ${ }^{4,26,32,33}$ ). 


\section{Chapter 7}

\section{Linear Braid Hybrid Carbon Fiber Composite}

\section{Structures}

Since the CFRP truss has a higher specific strength than foams, a better balance of properties might be achieved by increasing the volume fraction of the core occupied by trusses, while using the lowest density foam whose compressive strength was sufficient to preserve the trusses cross sectional shape. However, the complexity of the braiding process used to make a carbon fiber net made it difficult to increase the truss volume fraction, and to therefore investigate the effect of changing the truss volume fraction on the properties of the hybrid core. In this chapter, the use of a simpler linear carbon fiber braid approach to fabricate hybrid CFRP truss/foam core sandwich panels using a medium strength and density (Divinycell H250) foam is explored, the effect of varying the CFRP truss volume fraction within the core investigated. The truss relative density within the core unit cell could be easily changed by varying either the truss length $l$, or the diameter $d$, of the braid.

\subsection{Panel Design and Fabrication.}

\subsubsection{Hybrid Design}

The hybrid linear braid sandwich panel design is schematically illustrated in Figure 7.1 and was similar to that fabricated using the braided net approach (See Chapter 
5). Briefly, linear carbon fiber braids of different diameters were fabricated from the same IM7 carbon fiber used in the braided net study. These were utilized along with a medium density closed cell PVC (Divinycell H250) polymer foam to form a hybrid CFRP/ polymer foam structure. The linear braids formed the trusses within the core, and were stitched to 3-D woven face sheets using Kevlar thread. Divinycell foam has a density of $250 \mathrm{kgm}^{-3}$ and a compressive strength of $6 \mathrm{MPa}$; sufficient to reduce truss flattening during the consolidation process and to support the trusses after their initial fracture during compressive testing. The trapezoidal cross section foam molds were created by milling the Divinycell foam. They contained semi-circular cross section, variable diameter grooves for placement of the trusses and control of the truss fiber volume fraction.

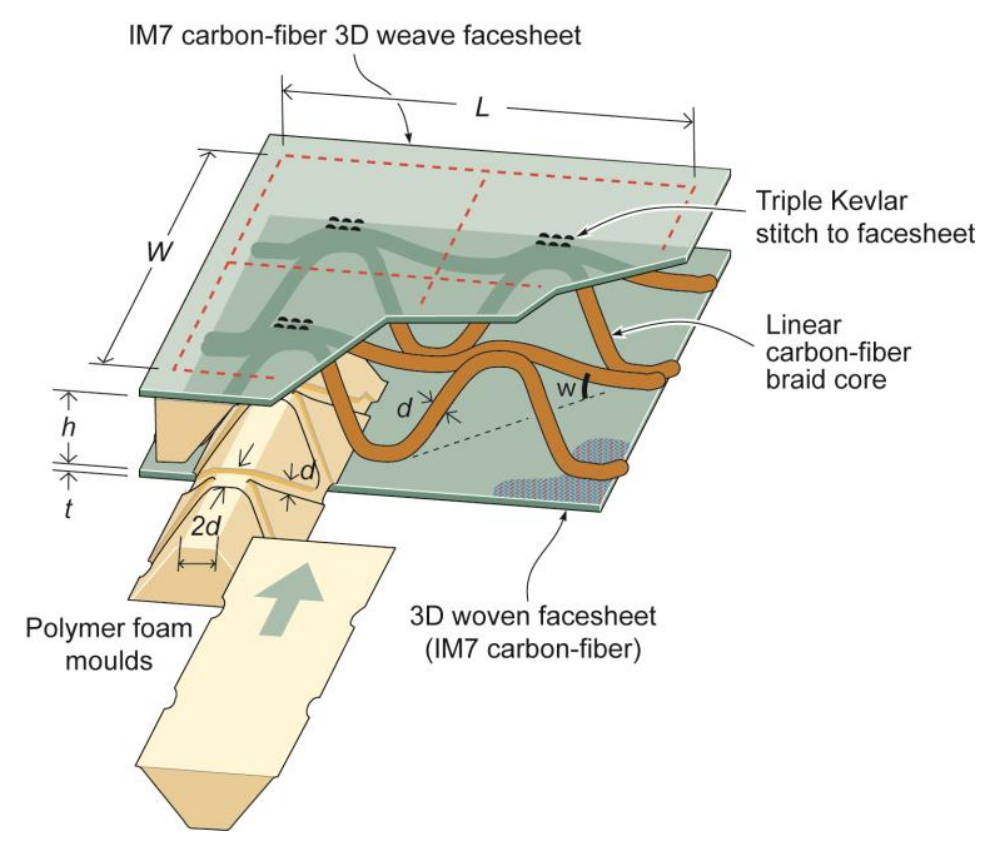

Figure 7.1: Hybrid composite core structure consisting linear carbon fiber braids with polymer foam inserts configured as the core of a sandwich panel with 3D woven carbon fiber composite face sheets. 


\subsubsection{Core Geometry}

A unit cell of the most general pyramidal lattice with elliptical cross-section trusses, is shown in Figure 7.2. The truss minor axis width (normal to the foam side surface) was defined as $d_{l}$ while its major axis length (parallel to the longitudinal axis of the mold) was $d_{2}$. The ellipticity ratio $\left(\mathrm{d}_{1} / \mathrm{d}_{2}\right)$ was approximately 0.86 for all the samples. The angle of inclination of the trusses to the base of the unit cell, $\omega$, determines the balance between out of plane compressive strength and in-plane shear resistance ${ }^{61}$. The structured investigated here used an angle of $45^{\text {oiii }}$. Preliminary trials indicated that simply folding a braid over the apex of a triangular foam mold led to dilation and ellipticity of the folded region, and resulted in low strength nodal failure of the braid during subsequent compression testing. This could be avoided by reducing the radius of curvature of the braid fold. The node at the truss/face sheet interface was therefore designed to have a width equal to twice the major axis diameter of the truss, and a length $b$ that was set equal to 2 times the woven diameter of the braid $d$ (see Table 7.1).

Four different linear braids were used to produce hybrid structures for this study. Their structure is illustrated, along with micrographs of the braids themselves, in Figures 7.3a-d. The four braids were fabricated by 3Tex (Cary, North Carolina) using 8, 16, 32, or 64 tows of 12k Hexcel IM7 carbon fiber, with a similar braiding angle of approximately $11^{\circ}$. The four linear braids have woven diameters of $2.7,3.8,5.2$, and $7 \mathrm{~mm}$, and repeat distances $\lambda=15,25,35$, and $65 \mathrm{~mm}$ respectively. The linear braids had a fiber volume fraction of approximately $50 \%$, and this volume fraction was maintained

\footnotetext{
iii This angle maximizes both the compressive and shear strength of the lattice. Increasing $\omega$ beyond $45^{\circ}$ increases the compressive strength while reducing the shear strength.
} 
for all the experimental studies reported here. The geometry and properties of these linear braids are summarized in Table 7.1.

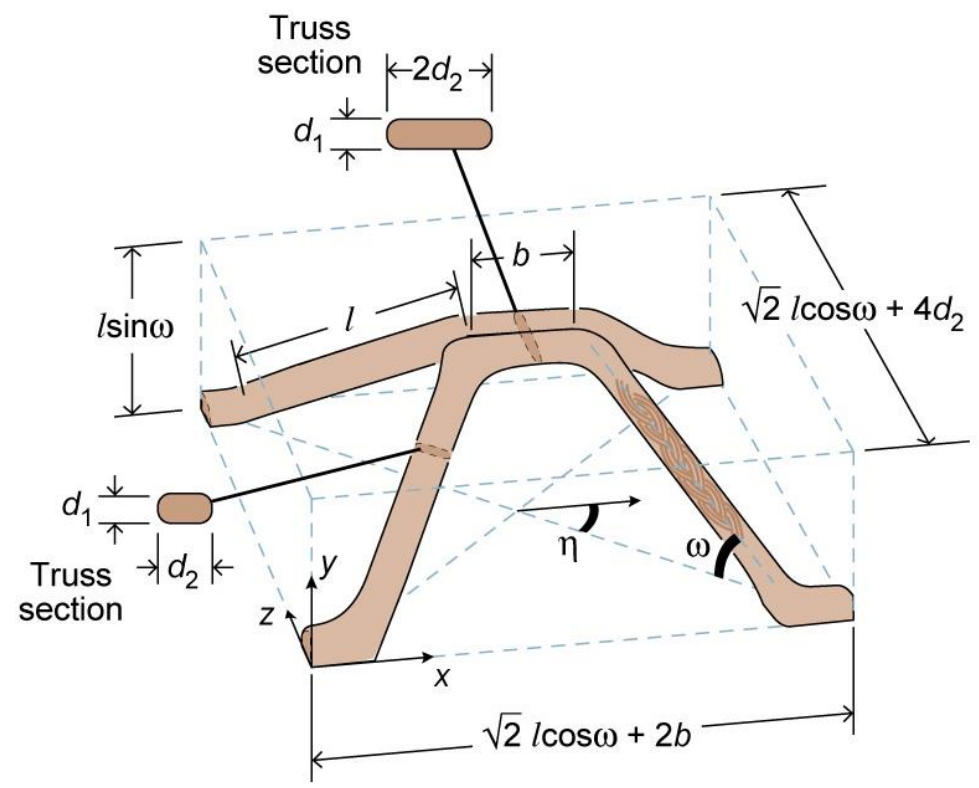

Figure 7.2: Pyramidal CFRP unit cell with elliptical cross section trusses. Two linear braids are required to form each unit cell. 
a 8 Tows

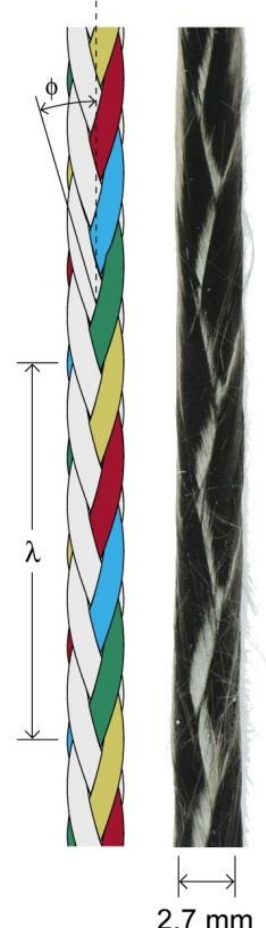

$27 \mathrm{~mm}$ b 16 Tows

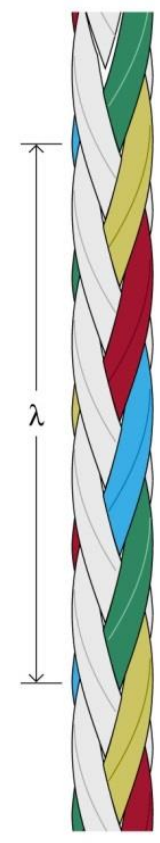

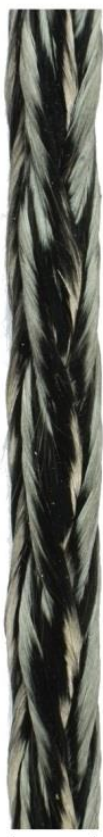

$\underset{3.8 \mathrm{~mm}}{\stackrel{\leftrightarrow}{\longrightarrow}}$ c 32 Tows
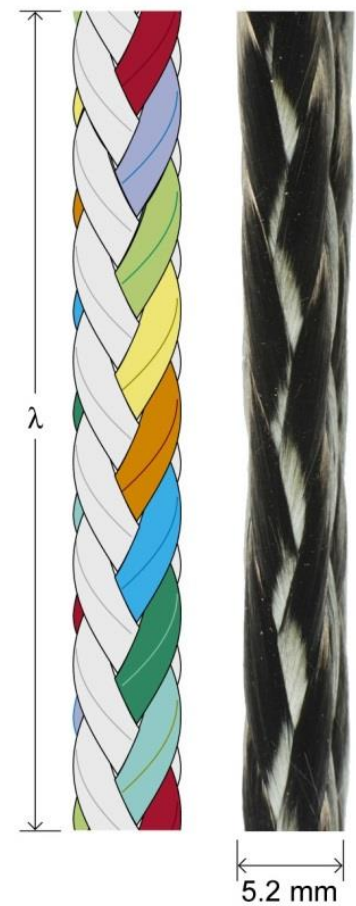

d 64 Tows
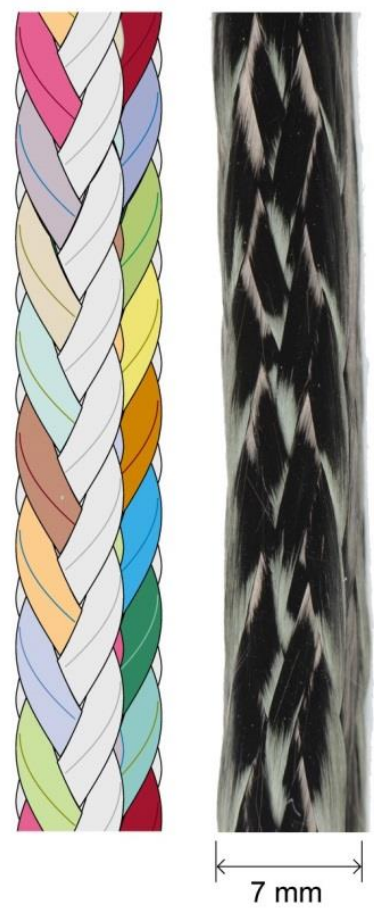

Figure 7.3: Illustrations of the structure of the linear braids together with photographs of the actual IM7 carbon fiber braids, containing (a) 8 tows, (b) 16 tows, (c) 32 tows, and (d) 64 tows. Each tow contained 12,000 carbon fibers.

Table 7.1: Summary of braid and CFRP strut properties

\begin{tabular}{|l|r|r|r|r|}
\hline Braid Number & 1 & 2 & 3 & 4 \\
\hline Number of tows per braid & 8 & 16 & 32 & 64 \\
\hline Braid Repeat Distance $\lambda(\mathrm{mm})$ & 15 & 25 & 35 & 65 \\
\hline Braid Angle (degree) & 11 & 11 & 11 & 11 \\
\hline Woven Braid Diameter (mm) & 2.7 & 3.8 & 5.2 & 7 \\
\hline Fiber Volume Fraction $v_{f}$ & 0.47 & 0.48 & 0.51 & 0.53 \\
\hline CFRP Strut Compressive Strength (MPa) & 570 & 555 & 550 & 530 \\
\hline CFRP Strut Compressive Modulus (GPa) & 39 & 37 & 37 & 35 \\
\hline CFRP Strut Tensile Strength (MPa) & 850 & 810 & 815 & 790 \\
\hline CFRP Strut Tensile Modulus (GPa) & 41 & 39.2 & 38.4 & 37.5 \\
\hline
\end{tabular}


Linear braids with 8,16 , and 32 tows were used to prepare hybrid cores with truss relative densities of $1.5,2.7$, and $4 \%$ respectively with $l$ fixed. The 64 tow braid was used to prepare cores with truss relative densities of $7,12.5$, and $17.5 \%$, by changing the truss length $l$. The values of $d_{1}$ and $d_{2}$ for the oval truss were determined for each of the linear braids using X-ray computed tomography (X-CT) measurements of the completed, untested panels. The core design parameters for the six core types are summarized in Table 7.2.

\subsubsection{Panel Assembly and Infiltration}

The assembly sequence used to fabricate the hybrid structure is schematically illustrated in Figure 7.4. The linear braids were first stitched to the dry face sheet at the base of one of the pre-machined foam molds. The linear braids are then placed within the mold's grooved channels, and an inverted foam mold used to enclose the braid within a circular channel equal to the dry braid diameter $(2.7,3.8,5.2$, and $7 \mathrm{~mm}$ for the $8,16,32$, and 64 tow linear braids respectively). The process was repeated until the desired number of pyramidal core lattice cells was created. Two linear braids were required for each row of the pyramidal lattice unit cells. The molds had a flat node region, the width of which was twice the woven diameter of the braid. The linear braids were stitched to the face sheet using three stitches of Kevlar thread over a length of approximately $15 \mathrm{~mm}$. The 3Weave ${ }^{\mathrm{TM}}$ face sheets were made from $12 \mathrm{~K}$ Hexcel IM7 carbon fiber tows by 3Tex Inc.

The structure of the $3 \mathrm{Weave}^{\mathrm{TM}}$ fabric is schematically illustrated in Figure 7.5a. The fabric consisted of 4 rows of weft fibers tows and 3 rows of orthogonal warp fiber tows, and a $\mathrm{z}$ yarn interpenetrating the layers, and running along the warp fiber direction. 
Approximately $47 \%$ of the fibers were in the weft direction, $43 \%$ in the warp direction, and $10 \%$ were $\mathrm{z}$ fibers. Figure $7.5 \mathrm{~b}$ shows a XCT image of the face sheet material, showing the as woven structure of the warp, weft, and $\mathrm{z}$ tows.

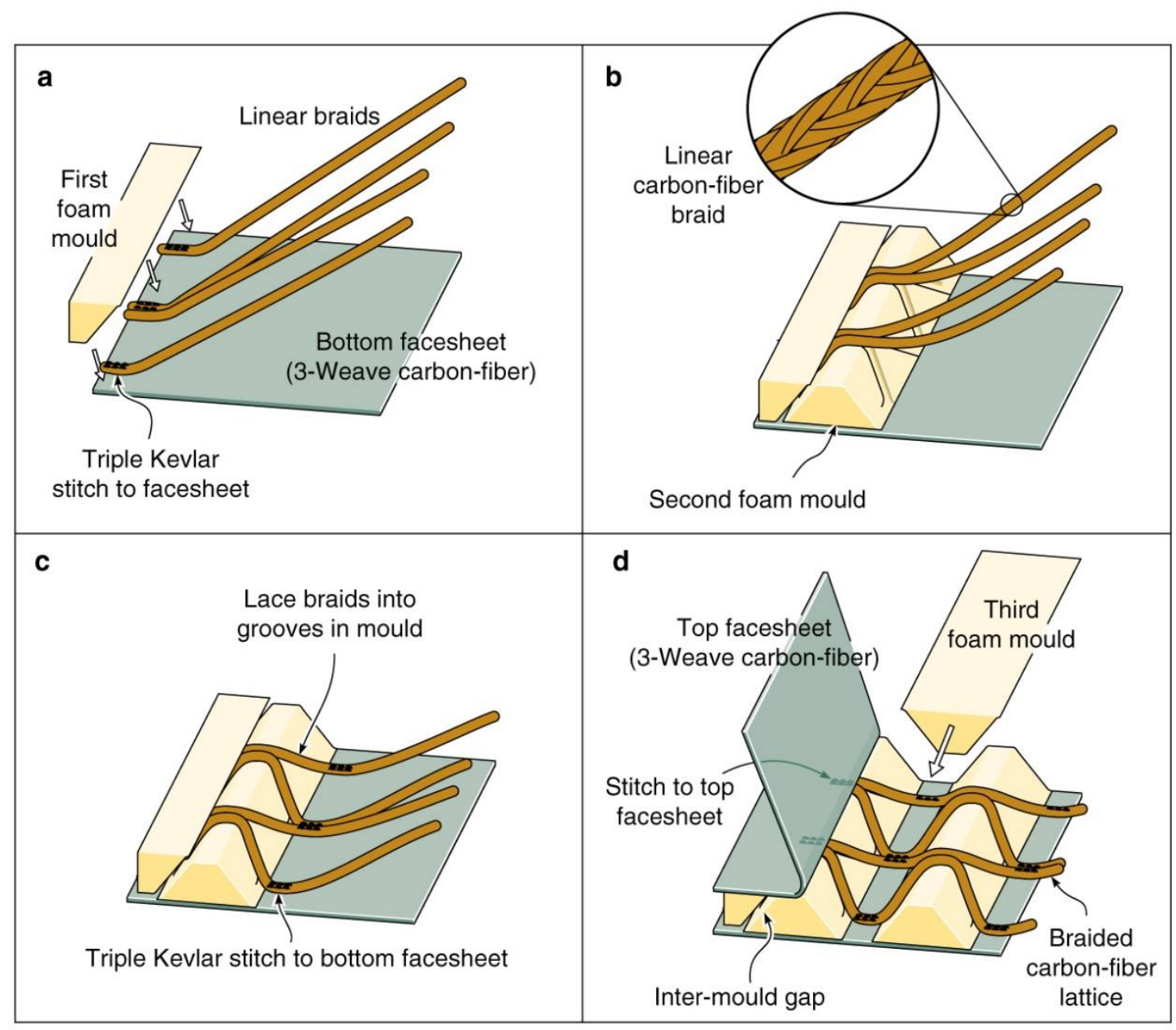

Figure 7.4: The linear hybrid lattice core sandwich panel assembly sequence. 


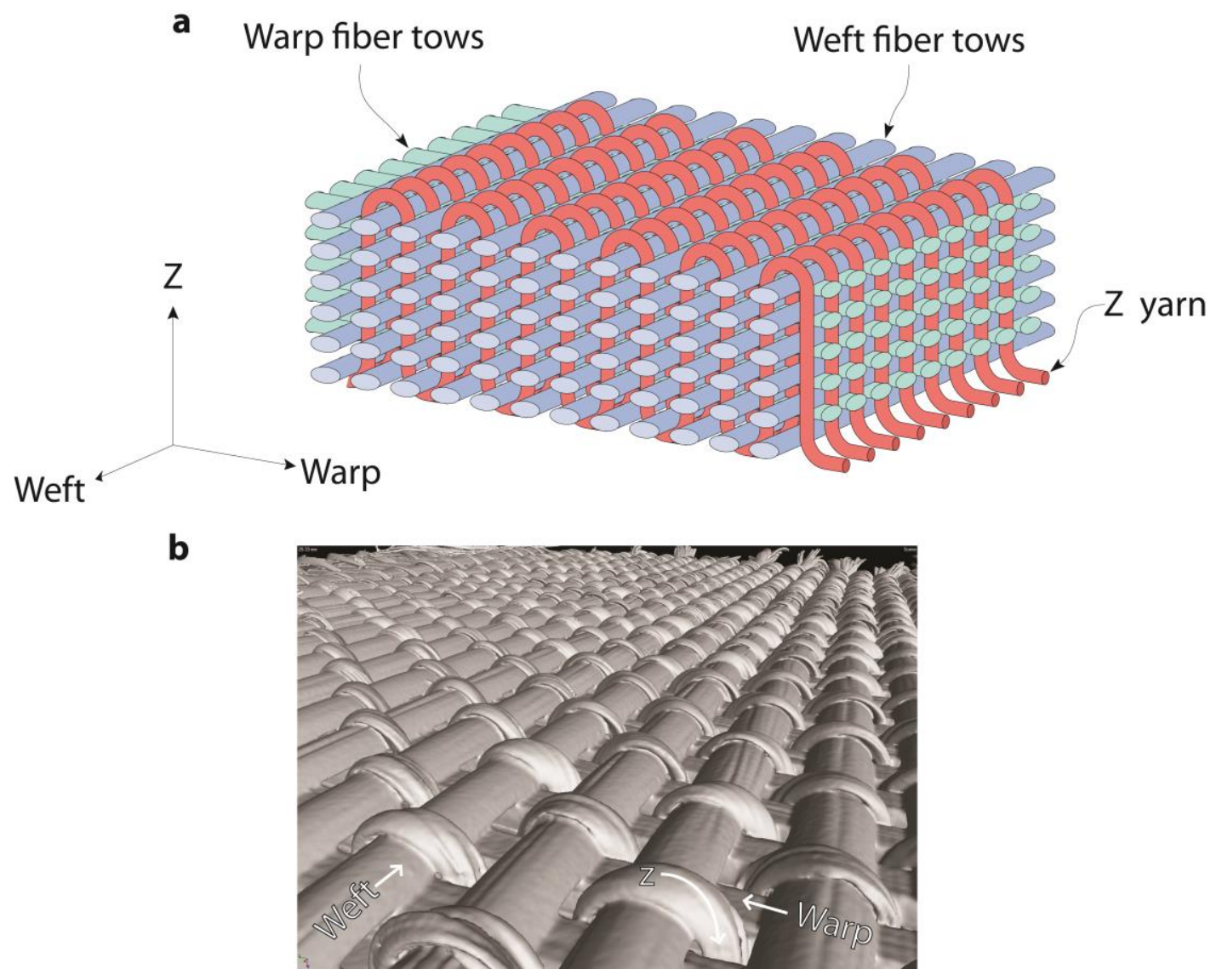

Figure 7.5: (a) Structure of the 3 Weave face sheet (b) Reconstructed XCT showing top view of the 3 Weave material.

After dry assembly, the panels were infiltrated with a SC1A epoxy resin and SC1B curing agent (Applied Poleramic Inc., Benicia, CA), with an epoxy to curing agent ratio of 100:22, using an vacuum assisted resin transfer molding process within an autoclave chamber (ASC Process Systems, Valencia, CA). This cured epoxy has a compressive strength of $75 \mathrm{MPa}$, and a Young's modulus $E_{\text {resin }}=1.85 \mathrm{GPa}$. After the cure cycle was complete, the CFRP panels were removed from the infusion tooling, and machined to the appropriate dimensions needed for testing. Each test panel comprised of 
four unit cells, in a $2 \mathrm{X} 2$ unit cell array. The geometry and other parameters of the composite panels are listed in Table 7.2. As discussed earlier (Chapter 5), small gaps between the prismatic foam inserts resulted in a thin layer of resin being retained between the foam molds of the hybrid core. Figure 7.6 shows reconstructed XCT images of 4 specimens where the trusses occupy $1.5,4,7$, and $17.5 \%$ of the core volume.

\subsubsection{Hybrid Core Density}

The relative density of the CFRP trusses within the core structure $\bar{\rho}$, including the contribution from the node, was found by calculating the ratio of the truss volume to that of the unit cell. One quarter of a node can be assigned to each lower corner of the unit cell, and a full node volume to the top face. For the most general case of an elliptical truss, the volume of the unit cell $\mathrm{V}$ is given by:

$$
V=l \sin \omega\left(\sqrt{2} l \cos \omega+4 d_{2}\right)(\sqrt{2} l \cos \omega+2 b)
$$

The relative density (volume fraction) of the truss within the core is then be obtained by dividing the truss volume by that of the unit cell:

$$
\bar{\rho}=\frac{\pi d_{1} d_{2}(l+b)}{l \sin \omega\left(\sqrt{2} l \cos \omega+4 d_{2}\right)(\sqrt{2} l \cos \omega+2 b)}
$$



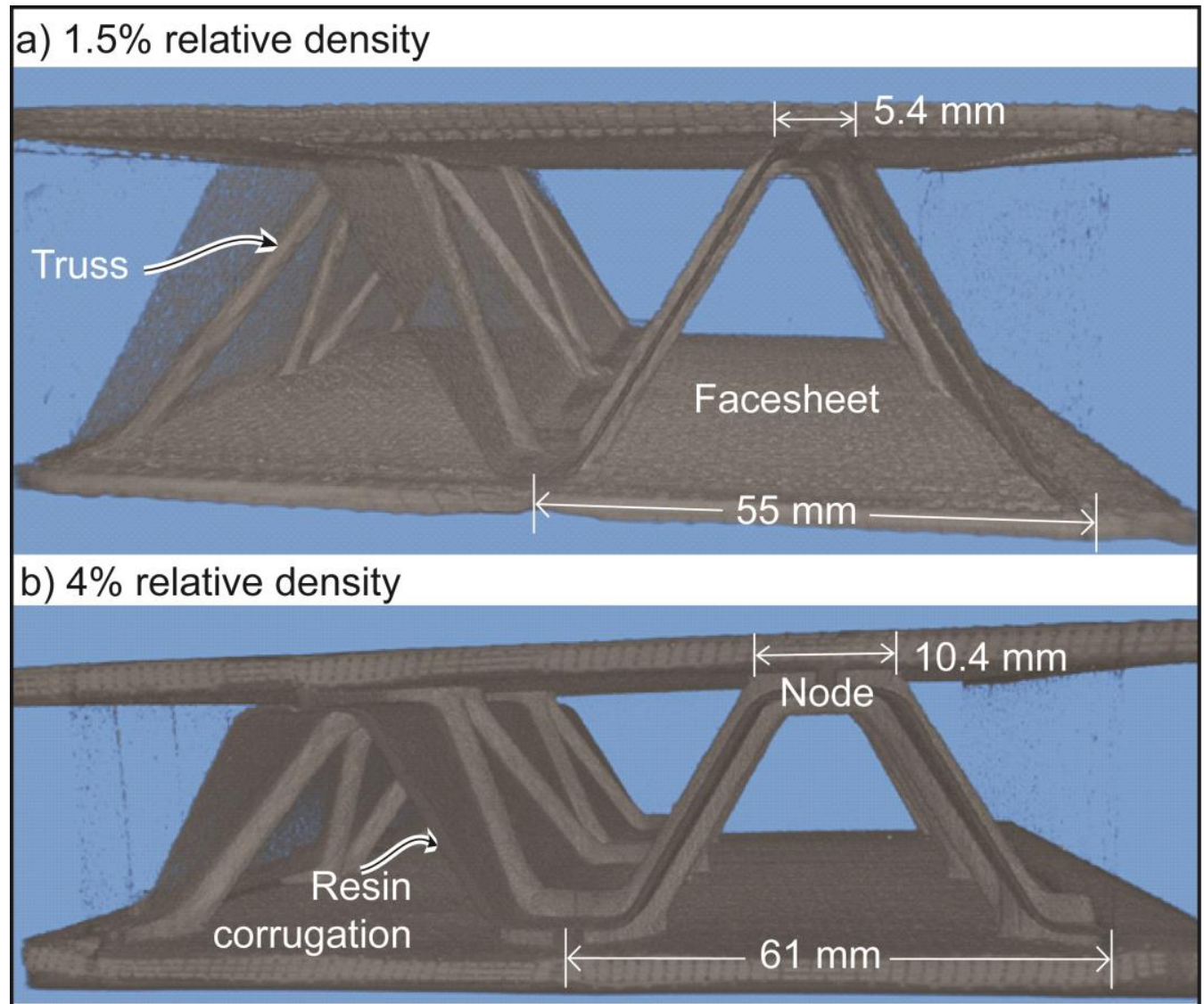

c) $7 \%$ relative density

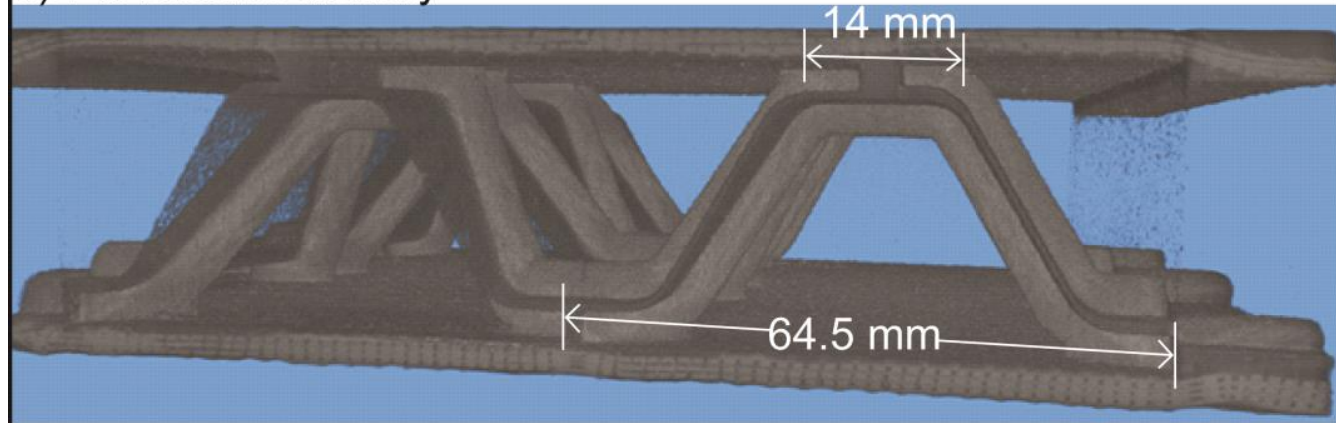

d) $17.5 \%$ relative density

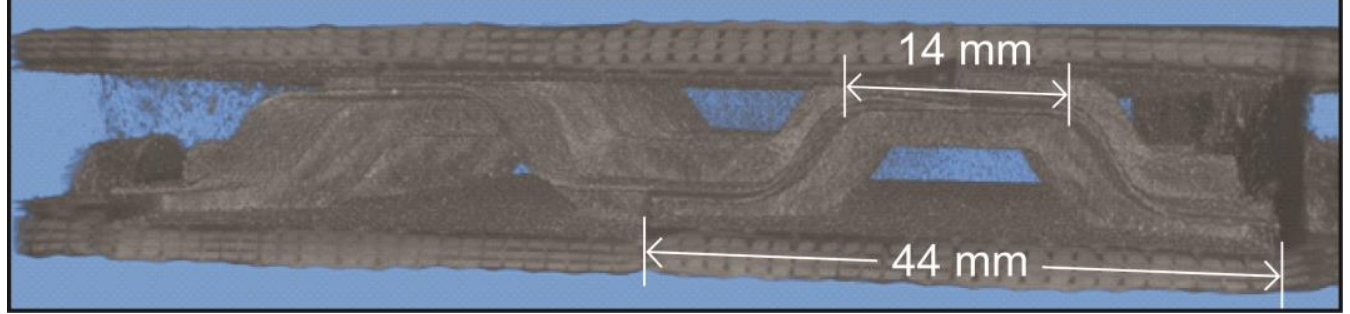

Figure 7.6: Reconstructed XCT images showing an untested specimen with the CFRP truss comprising: a) $1.5 \%$; b) $4 \%$, c) $7 \%$, and d) $17.5 \%$ of the core. 
The density of the hybrid core can be deduced by summing the contribution of the components of the core. The total density of the hybrid core, $\rho$, is found by accounting for the masses of the foam and resin sheets in a unit cell. The volume, $v_{r s}$ occupied by a resin sheet of thickness $t$ within the unit cell was estimated by calculating the volume of the corrugated resin sheet, including the flat region near the nodes:

$$
v_{r s}=2 t(l+b)(\sqrt{2} l \cos \omega+4 d)
$$

where $l$ is the truss length, $\omega$ is the truss inclination angle, $b$ is the node length, $d_{l}$ is the short axis diameter of the truss, and $d_{2}$ is the long axis diameter of the truss (See Figure 7.2). The volume fraction of the resin sheet $v_{f r}$ is found by dividing Equation 7.2 by the volume of the unit cell (Equation 7.1a):

$$
v_{f r}=\frac{2 t(l+b)}{l \sin \omega(\sqrt{2} l \cos \omega+2 b)}
$$

The thickness of the resin sheet measured by XCT for all samples had an average value $t_{\text {avg }} \approx 0.5 \mathrm{~mm}$. It was assumed that the volume within the unit cell which was not occupied either by the truss or the resin sheet was occupied by foam. The volume fraction of the foam $v_{f f}$ can then be estimated as:

$$
v_{f f}=\left(1-\left(\bar{\rho}+v_{f r}\right)\right)
$$

Where $\bar{\rho}$ is given by Equation $1 \mathrm{~b}$, and $v_{f r}$ (Equation 7.3 ). The total density of the hybrid core, $\rho$ can is then:

$$
\rho=\left(\bar{\rho} \rho_{c c}\right)+\left(\rho_{r s} v_{r s}\right)+\left(\rho_{f} v_{f f}\right)
$$


where, $\rho_{c c}$ is the measured density of the braided CFRP trusses $\left(1430 \mathrm{kgm}^{-3}\right), \rho_{r s}$ is that of the cured epoxy resin $\left(1100 \mathrm{kgm}^{-3}\right)$, and $\rho_{f}$ is the density of the Divinycell $\mathrm{H} 250$ foam $\left(250 \mathrm{kgm}^{-3}\right)$. The density of the hybrid core panels is given in Table 7.2.

\subsection{Mechanical Properties of Linear Braided Truss}

When a pyramidal lattice structure made using linear braids is subjected to out of plane compressive or an in plane shear loads, the trusses experience either axial tensile or compressive forces. The axial mechanical properties of the linear braid truss in axial compression and tension therefore govern the lattice response, and have been measured here.

\subsubsection{Axial Compression}

The axial compressive strength of each of the four linear braids was determined by preparing circular cross section CFRP truss samples from each of the braids confined within circular glass tubes with inner diameters of $\mathrm{d}=2.7,3.8,5.2$, and $7 \mathrm{~mm}$ for the linear braids with $8,16,32$, and 64 tows respectively. These inner tube diameters resulted in carbon fiber volume fractions that were similar (about $50 \mathrm{vol} \%$ ) to those of the truss structures in the hybrid cores, Table 7.1. Five specimens were tested for each of the linear braids. (Same number of specimens tested in tension) The resin infiltrated structures were cured, and the tubing removed, leaving behind the cylindrical braided composite samples. The cylindrical samples were then cut into $20 \mathrm{~mm}$ long pieces, which were sufficiently stocky to ensure avoidance of Euler buckling, their ends coated with SCI epoxy to prevent brooming failure ${ }^{62}$, attached to slotted tabs and tested in compression at ambient temperature $\left(23^{\circ} \mathrm{C}\right)$ at a strain rate of $2 \times 10^{-4} \mathrm{~s}^{-1}$. The elastic 
modulus for each sample was measured by unloading within the nominally elastic region of the stress-strain curve.

The compressive stress-strain responses for each linear braid truss type are shown in Figure 7.7, and their average moduli and strengths summarized in Table 7.1. All of the linear braid samples failed by a fiber microbuckling mechanism that was initiated in one of the tows and then propagated on an inclined plane across the sample. The compressive strengths of the four linear braid composites cylinders were similar, lying between 530 and $570 \mathrm{MPa}$.
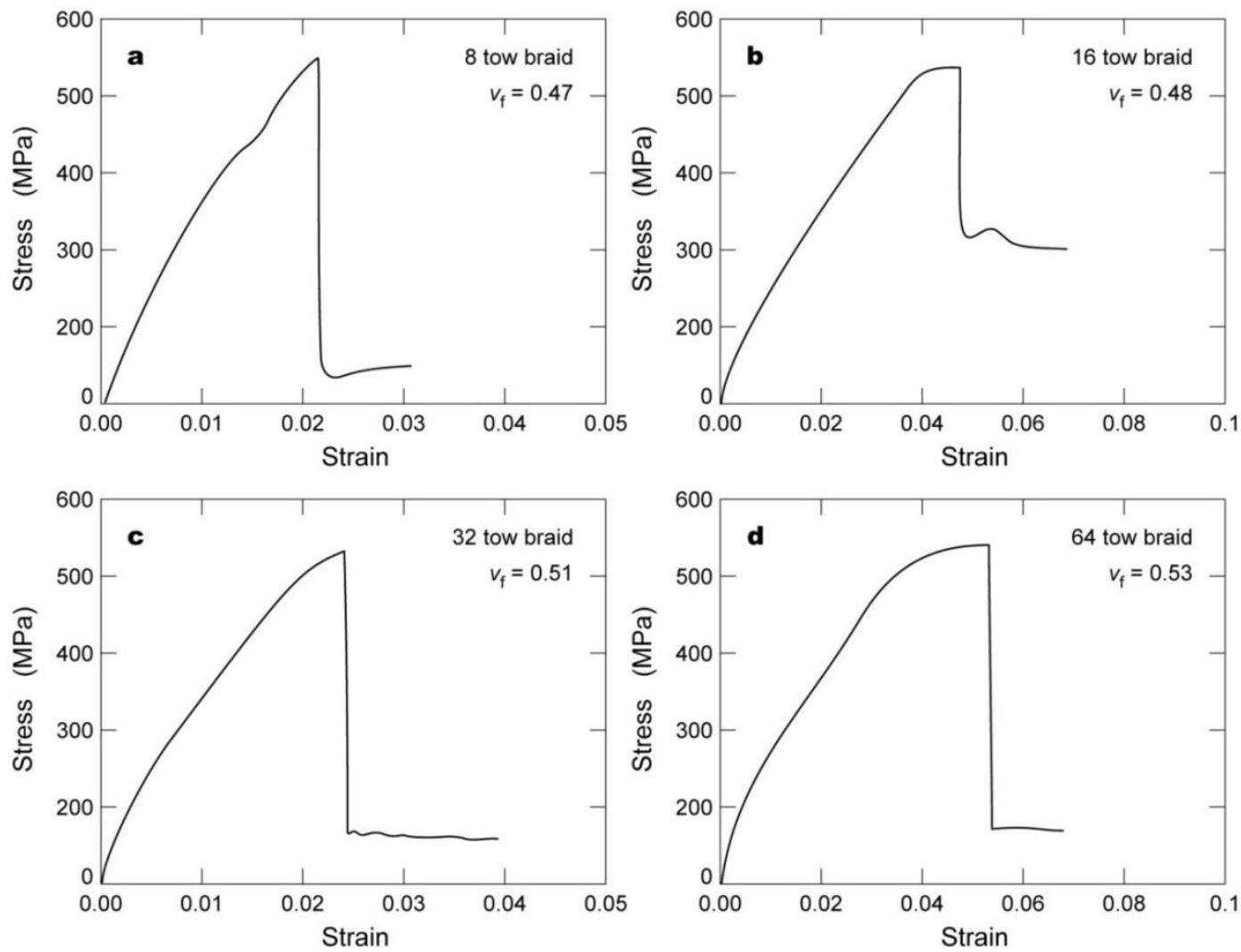

Figure 7.7: Typical compressive stress-strain response for each of the infused linear braids tested. The specimens tested were all $20 \mathrm{~mm}$ in length, all fail by fiber microbuckling. 


\subsubsection{Tension}

The tensile strength of the braided composite cylinders was determined in accordance with the ASTM D3039 standard for composite tensile testing. The samples had a $50 \mathrm{~mm}$ gauge length, and were then strained at a constant rate of $2 \times 10^{-4} \mathrm{~s}^{-1}$ at ambient room temperature $\left(23^{\circ} \mathrm{C}\right)$. The specimens failed by tensile fracture within the sample gauge length. The stress strain curves for a typical specimen of each of the linear braids is shown in Figure 7.8, and the average tensile strength and modulus for each of the linear braid composites is summarized in Table 7.1. The tensile strength and modulus of the composite cylinders are substantially lower than expected from a rule of mixtures calculation for unidirectional fibers, and the factors contributing to strength loss include damage to the fibers during the weaving process, reduction of strength at flaws where tows are severely distorted in the composite, and the uneven distribution of loads due to random tow waviness, effects which have been previously reported in literature ${ }^{63}$.

Table 7.2: Hybrid core properties

\begin{tabular}{|l|r|r|r|r|r|r|}
\hline Truss relative density $(\%)$ & 1.5 & 2.7 & 4 & 7 & 12.5 & 17.5 \\
\hline Braid used & 1 & 2 & 3 & 4 & 4 & 4 \\
\hline Truss diameter as designed $(\mathrm{mm})$ & 2.7 & 3.8 & 5.2 & 7 & 7 & 7 \\
\hline Strut ellipticity $\mathrm{d}_{1} / \mathrm{d}_{2}(\mathrm{~mm} / \mathrm{mm})$ & $2.3 / 2.7$ & $3.3 / 3.8$ & $4.5 / 5 / 2$ & $6.1 / 7$ & $6.1 / 7$ & $6.1 / 7$ \\
\hline Node length $b(\mathrm{~mm})$ & 5.4 & 7.6 & 10.4 & 14 & 14 & 14 \\
\hline Truss node volume fraction $(\%)$ & 11.9 & 15.9 & 20.6 & 20.7 & 29.4 & 35.4 \\
\hline Truss length $(\mathrm{mm})$ & 40 & 40 & 40 & 40 & 24.5 & 19 \\
\hline Bent fractional truss length $x$ & 0.75 & 0.73 & 0.72 & 0.72 & 0.78 & 0.81 \\
\hline Densification strain $\varepsilon_{\mathrm{D}}$ & 0.59 & 0.58 & 0.57 & 0.52 & 0.37 & 0.30 \\
\hline Resin sheet thickness $t_{\text {avg }}\left(\mathrm{mm}^{2}\right)$ & 0.45 & 0.47 & 0.51 & 0.55 & 0.57 & 0.6 \\
\hline Core density calculated $\left(\mathrm{kgm}^{-3}\right)$ & 271 & 285 & 299 & 336 & 398 & 474 \\
\hline Core density measured $\left(\mathrm{kgm}^{-3}\right)$ & 298 & 312 & 328 & 364 & 430 & 490 \\
\hline Core Compressive Strength $(\mathrm{MPa})$ & 7.7 & 9.6 & 12 & 14 & 22 & 25 \\
\hline Core Compressive Modulus $(\mathrm{MPa})$ & 390 & 550 & 710 & 1010 & 1450 & 1850 \\
\hline
\end{tabular}



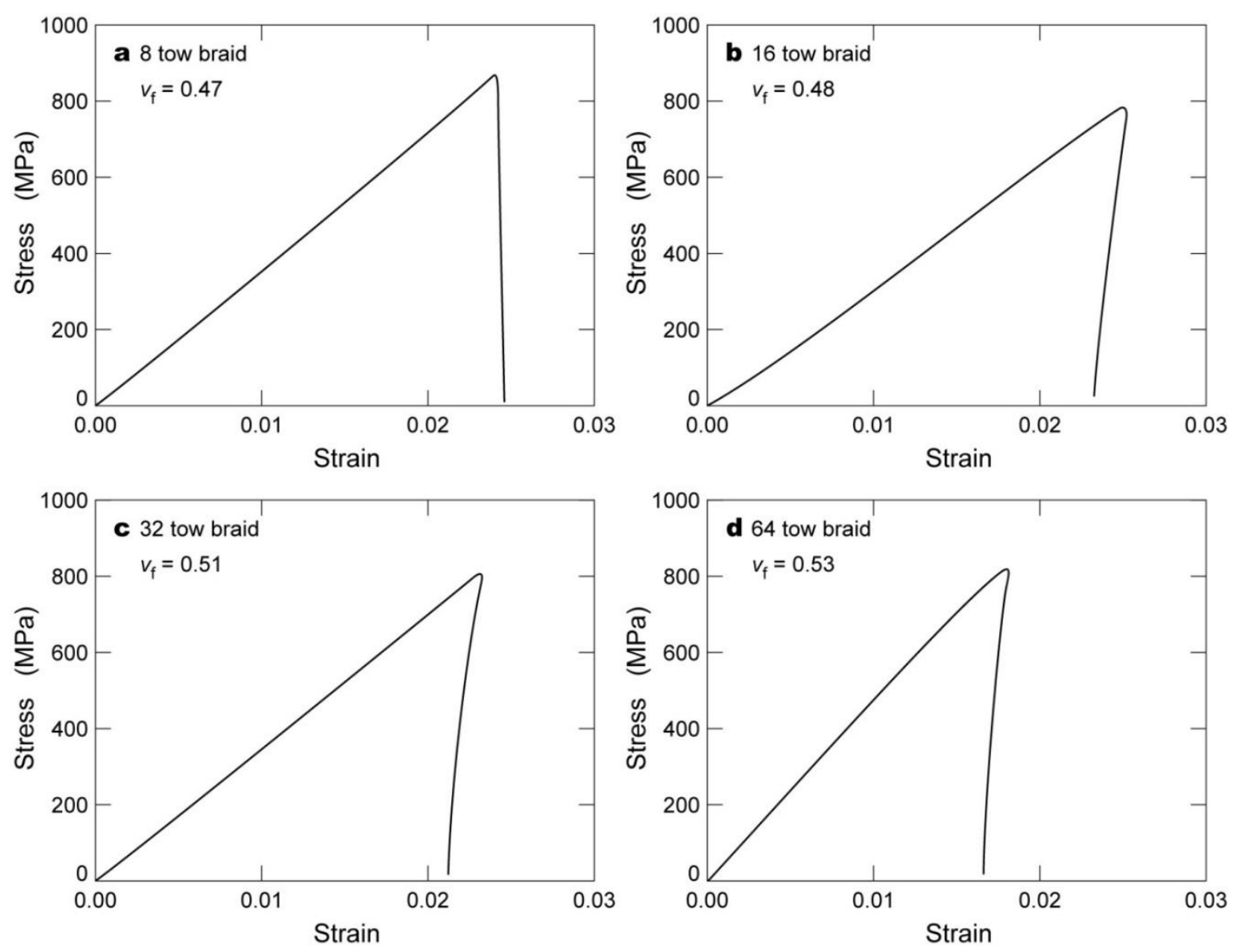

Figure 7.8: Typical tensile stress-strain responses for each of the linear braid composites tested.

\subsection{Hybrid Core Compression Response}

The through thickness compressive response of the sandwich panels was measured with a screw-driven universal testing machine (Model 4208 Instron Corporation, Canton, MA) with a 300kN load cell in accordance with ASTM C-365.

Retro-reflective tabs were attached to the top and bottom face sheets, and strain 
measurements were taken using a laser extensometer. The samples were compressed at a nominal strain rate of $2 \times 10^{-4} \mathrm{~s}^{-1}$ at room temperature $\left(23^{\circ} \mathrm{C}\right)$.

The compressive stress strain responses for the hybrid composite cores for each of the truss relative densities explored is shown in Figure 7.9. One specimen was tested for each core density. It can be seen that the stress strain curves for all the samples were initially linear, followed by yielding, a small rise in strength, a slight, sample dependent drop in strength followed by a stress plateau before the stress began to gradually rise as the core densified. The elastic modulus for each sample was measured by unloading within the nominally elastic region of the stress-strain curve. Figure 7.10a shows the dependence of the compressive modulus upon the density of the hybrid core. Figure 7.10b shows a plot of the compressive strength versus density for the linear braid composite hybrid cores. It can also be seen that both the elastic modulus and the yield strength increased as the truss relative density, or volume fraction of truss within the unit cell increased.

Figure 7.11 shows a sequence of isometric X-CT images of a hybrid composite panel with $7 \%$ truss volume fraction hybrid core for the as fabricated specimen (Figure 7.11a) after 10 and $50 \%$ strain total strain. Figure 7.12 shows alternate views of the same data revealing the axial cross section of the trusses at the same strain levels. It can be seen that the trusses fail by microbuckling on a shear plane near the nodes. The X-CT images also show that the resin sheets collapse by buckling. 

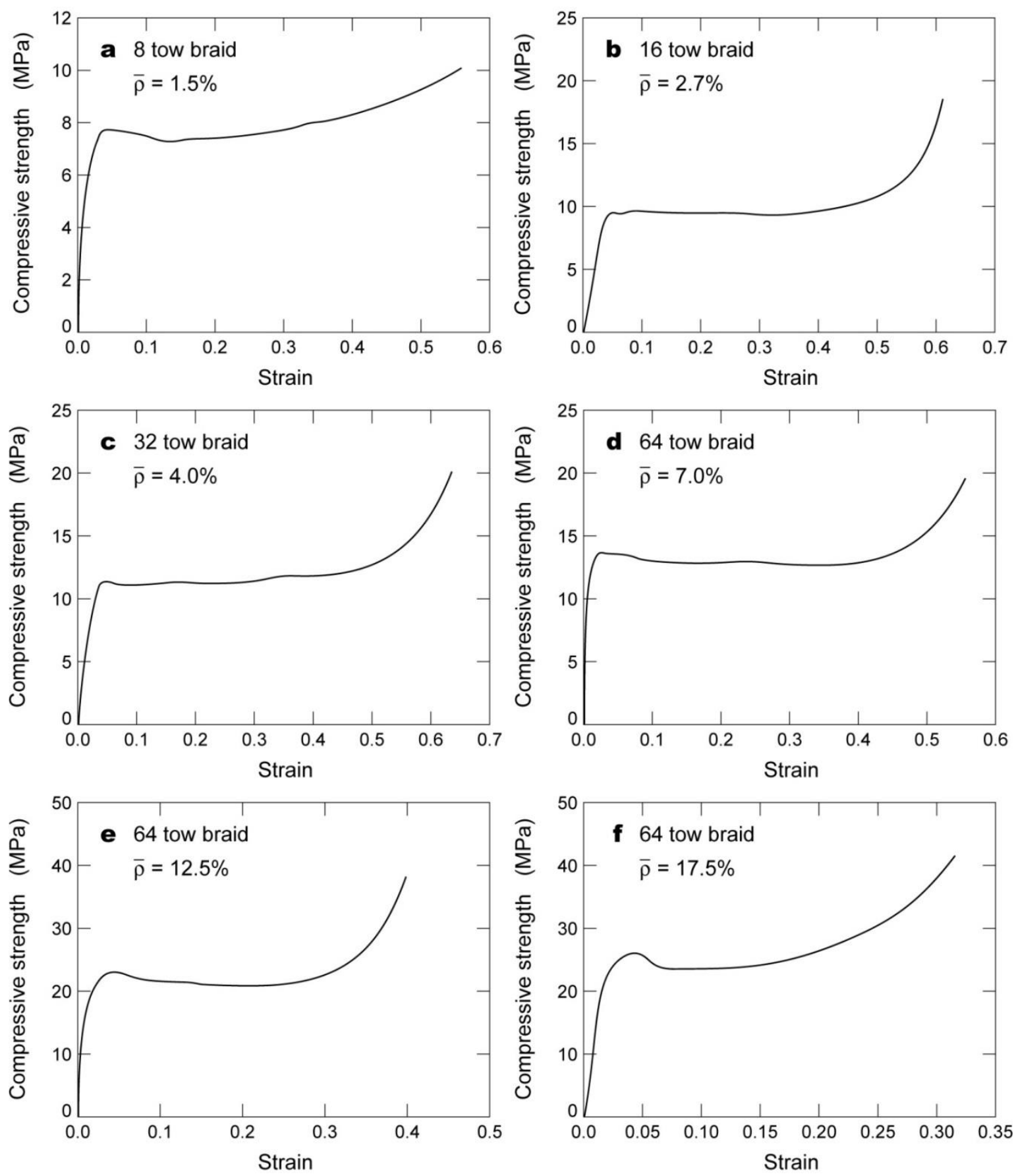

Figure 7.9: Compressive stress-strain curves for hybrid linear braid panels. 

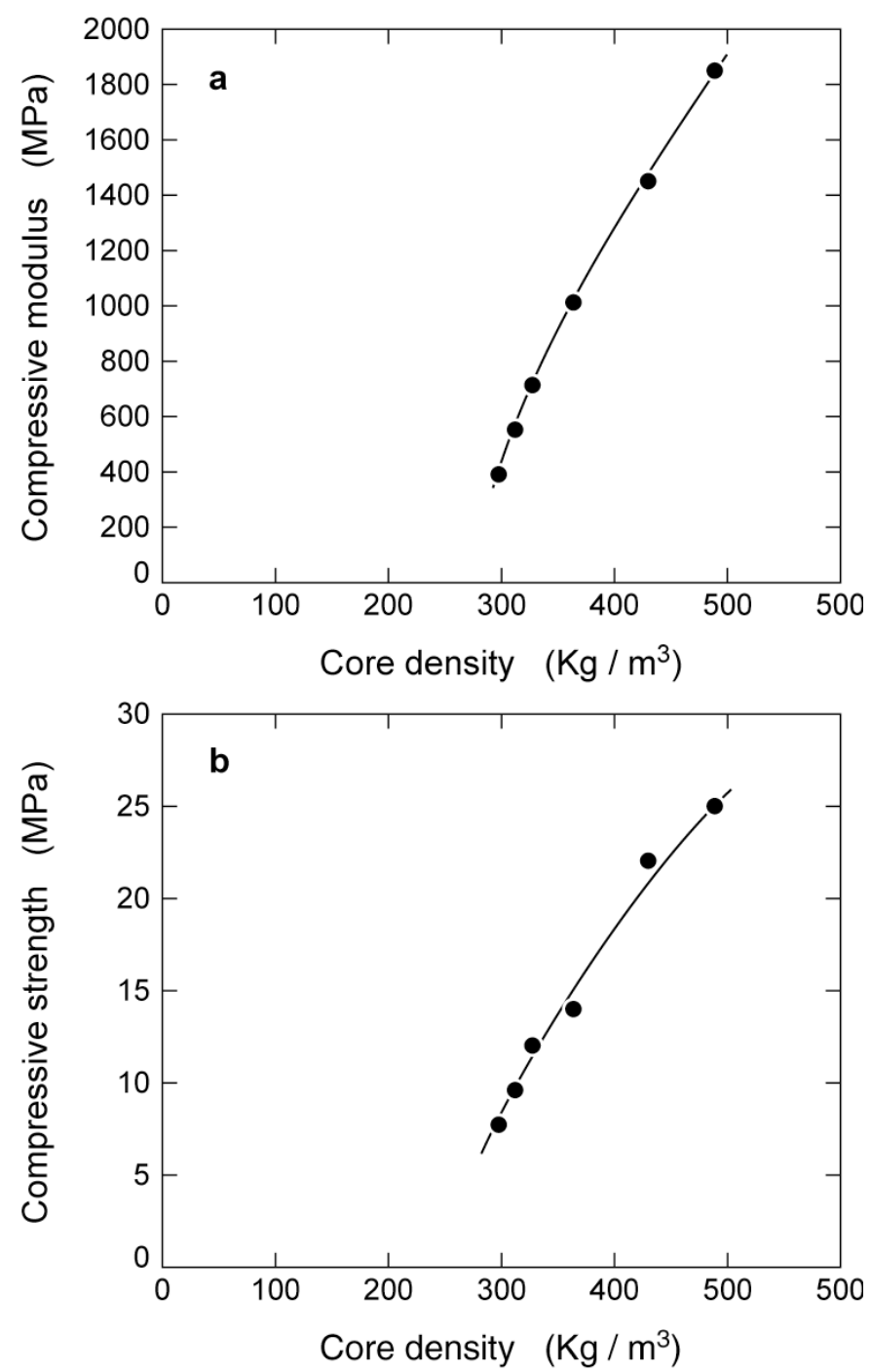

Figure 7.10: (a) The compressive modulus of the hybrid cores plotted against density (b) The compressive strength of the hybrid cores plotted against density. 

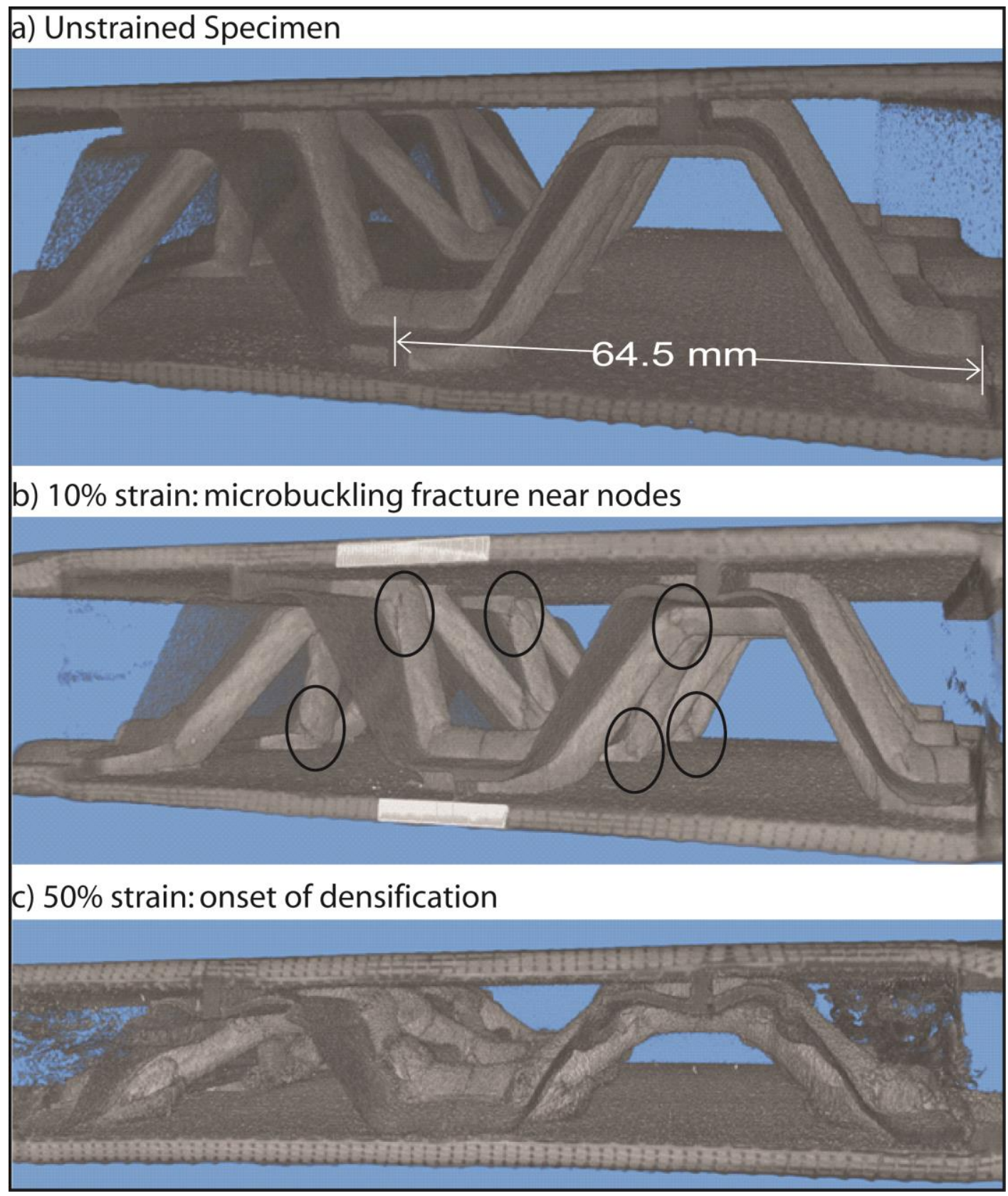

Figure 7.11: Reconstructed X-CT images of a hybrid composite panel with $7 \%$ truss volume fraction core (hybrid density of $364 \mathrm{~kg} / \mathrm{m}^{3}$ ): (a) as fabricated , (b)strained 10\%, and (c) strained 50\%. The trusses fail by fiber microbuckling at the circled locations and shear fracture near the nodes while the resin sheets fail by buckling. 


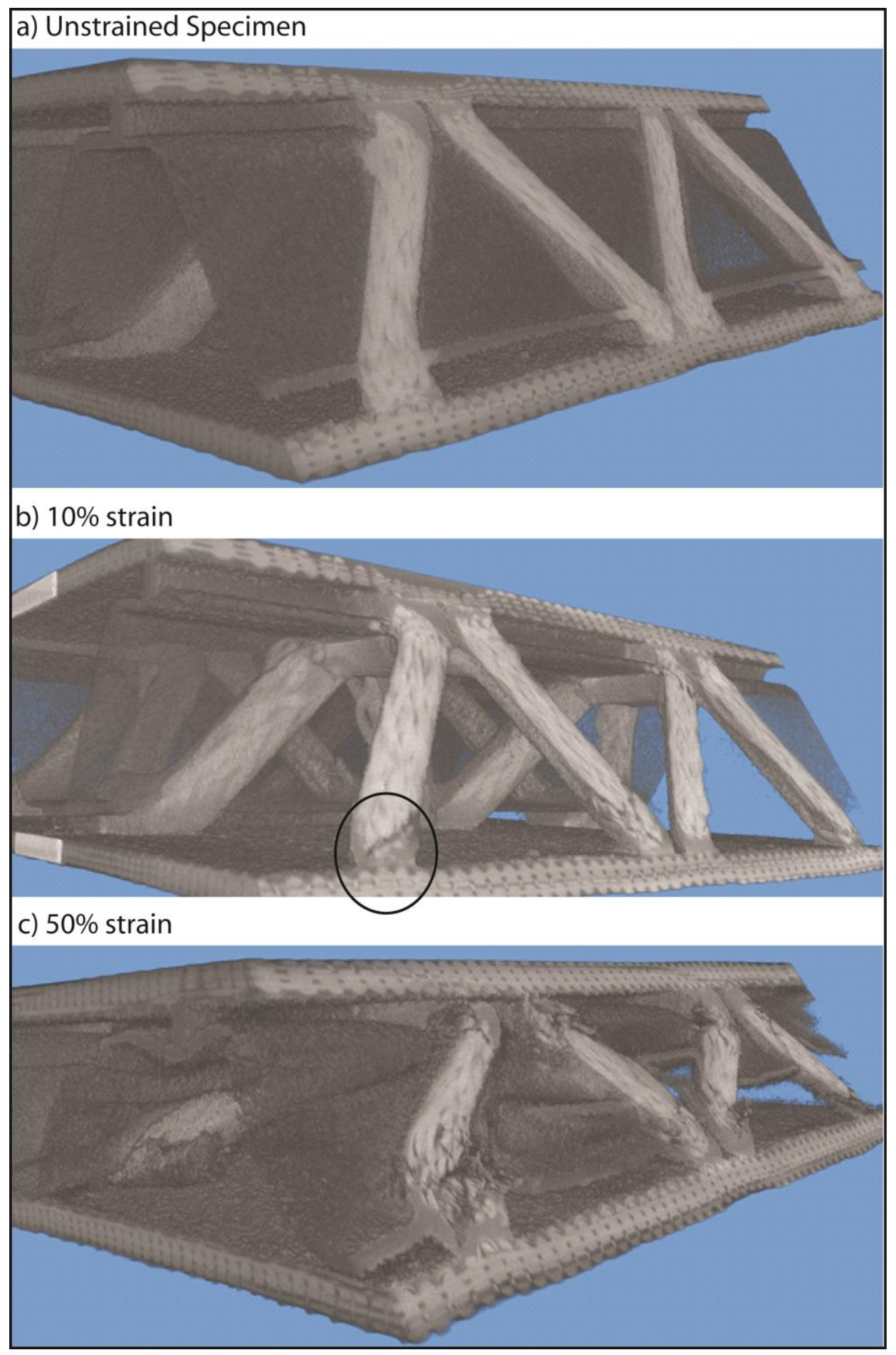

Figure 7.12: Reconstructed X-CT images of a hybrid composite panel with $7 \%$ truss volume fraction showing a longitudinal cross-sectional slice of the trusses (a) as fabricated state, (b) after $10 \%$ strain, and (c) after 50\% strain. 


\subsection{Micromechanical Analysis}

The experimental study indicates that the materials used to form the linear CFRP braid hybrid core and the geometry of the unit cell both influence the compressive modulus and strength of the core. The analysis of through thickness compression of a braided net hybrid core presented in Chapter 6 also applies here. It was shown that the elastic modulus of an empty CFRP truss lattice, $\mathrm{E}_{\text {lattice, }}$ with elliptical cross-section trusses, and no foam or resin corrugation, can be expressed in terms of the modulus of the CFRP axially compressed truss, $\mathrm{E}_{\text {truss, }}$, and the geometry of the unit cell:

$$
\frac{E_{\text {lattice }}}{E_{\text {truss }}}=\frac{8 \pi r_{1} r_{2} \sin \omega\left[\sin ^{2} \omega+\left(\frac{r_{1}^{2}}{l^{2}}\right) \cos ^{2} \omega\right]}{\left(\sqrt{2} l \cos \omega+4 d_{2}\right)(\sqrt{2} l \cos \omega+2 b)}
$$

where $d_{l}, d_{2}, l, \omega$, and $b$ are the unit cell parameters defined in Figure 7.2, and $r_{l}=d_{l} / 2$, and $r_{2}=d_{2} / 2$. The elastic modulus of hybrid core, $E_{c}$, can be estimated by assuming isostrain conditions whereupon the rule of mixtures gives:

$$
E_{c}=E_{\text {lattice }}+v_{f f} E_{\text {foam }}+v_{f r} E_{\text {resin }} \sin ^{4} \theta
$$

where $v_{f f}$ is the volume fraction of foam, and $v_{f r}$ is the volume fraction of the resin corrugation, and $\theta$ is the angle of inclination of the resin corrugation $\left(60^{\circ}\right)$. Using the geometry parameters for the hybrid cores summarized in Table 7.1, the three modulus components of Equation 7.7 for each of the core densities investigated here are shown in Figure 7.13b, and compared with the measured values in Figure 7.13a. It can be seen that there is good agreement between the predicted modulus and the measured values. Since the braided CFRP truss has a higher specific stiffness than either the foam or the resin 
sheet corrugation, the truss component of the modulus increases rapidly as the core density (truss volume fraction) increased.
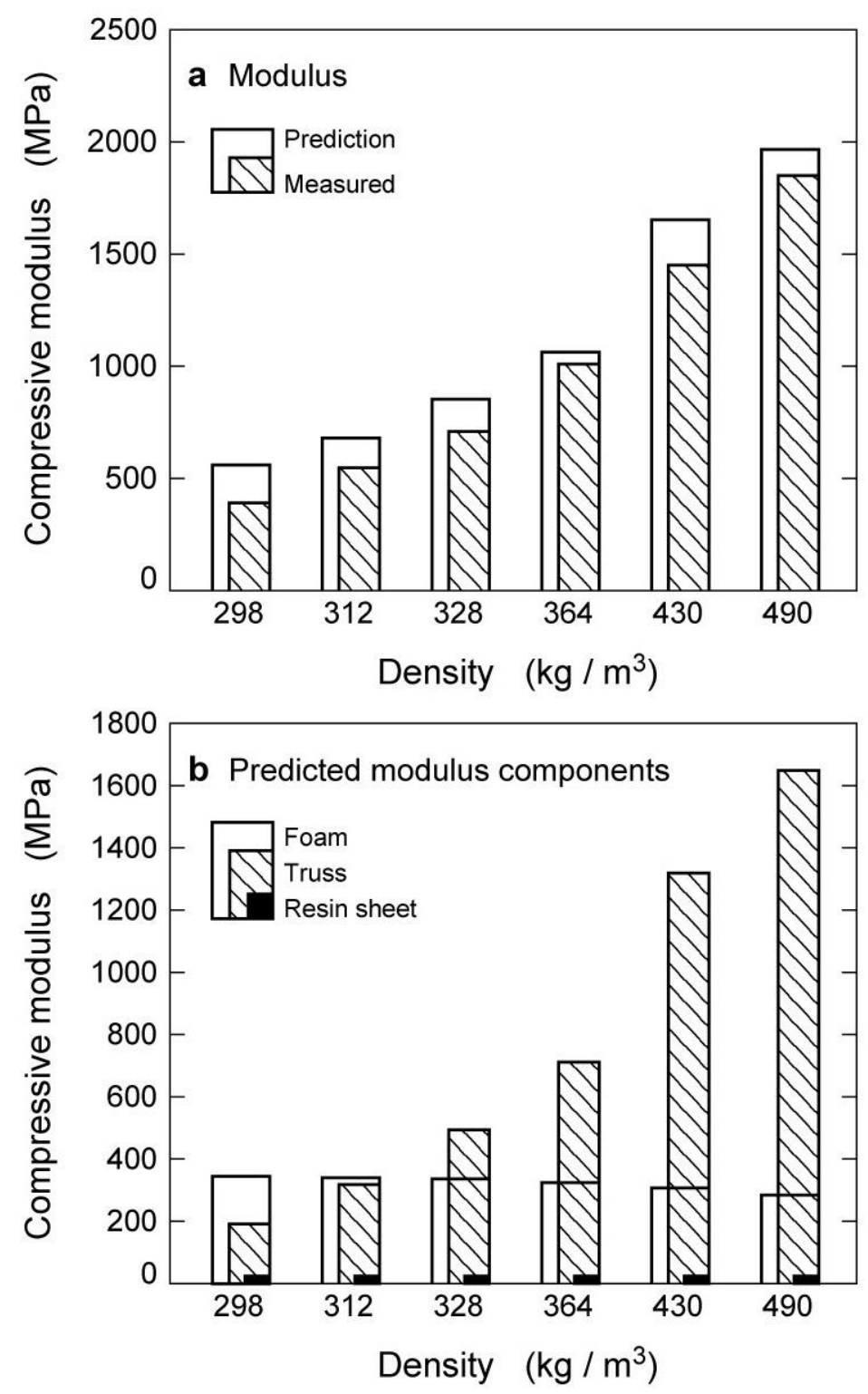

Figure 7.13: (a) The measured compressive modulus compared to that predicted for the different hybrid core densities, and (b) the individual modulus components of the prediction for each hybrid density. 
There are a number of potential failure modes for a CFRP truss, including fiber microbuckling, delamination, brooming, and elastic buckling. The braided and continuous nature of the truss ensured that delamination and brooming were not operative failure modes. Thus, the compressive strength of the pyramidal lattice within the hybrid core was controlled by either fiber microbuckling or global elastic (Euler) buckling depending upon whichever was lower for a given core type. The microbuckling strength of the pyramidal lattice, $\sigma_{\mathrm{p}}$, can be expressed in terms of the axial compressive strength of the CFRP truss, $\sigma_{\max }$, and unit cell parameters:

$$
\frac{\sigma_{p}}{\sigma_{\max }}=\frac{8 \pi r_{1} r_{2} \sin \omega}{\left(\sqrt{2} l \cos \omega+4 d_{2}\right)(\sqrt{2} l \cos \omega+2 b)\left[1+\left(\frac{r_{1}}{l}\right)^{2} \frac{\cot \omega}{\varphi}\right]}\left[\sin ^{2} \omega+\left(\frac{r_{1}}{l}\right)^{2} \cos ^{2} \omega\right]
$$

where $\sigma_{\max }$ is the compressive strength of the struts measured in Section 7.4.

The elastic buckling strength of the pyramidal lattice can likewise be expressed as:

$$
\frac{\sigma_{p}}{\sigma_{e l}}=\frac{8 \pi r_{1} r_{2} \sin \omega}{\left(\sqrt{2} l \cos \omega+4 d_{2}\right)(\sqrt{2} l \cos \omega+2 b)\left[1+\left(\frac{r_{1}}{l}\right)^{2} \frac{\cot \omega}{\varphi}\right]}\left[\sin ^{2} \omega+\left(\frac{r_{1}}{l}\right)^{2} \cos ^{2} \omega\right]
$$

where $\sigma_{\mathrm{el}}$ is the critical elastic buckling strength of the truss, which, for an elliptical cross section truss, is given by:

$$
\sigma_{e l}=\frac{\pi^{2} r_{1}^{3} r_{2} E_{t r u s s}}{4 l^{2}}
$$


The compressive strength of the foam filled hybrid core can then be predicted by assuming iso-strain conditions in the lattice, foam and polymer corrugation. The rule of mixtures compressive strength of the hybrid core can then be written as:

$$
\sigma_{h c}=v_{f f} \sigma_{f}+v_{f r} \sigma_{r} \sin ^{2} \theta+\sigma_{p}
$$

where $v_{f f}$ and, $v_{f r}$ are defined in Section 7.2.3, $\theta$ is the angle of inclination of the resin sheets, and $\sigma_{f}$ and $\sigma_{r}$ are the compressive strengths of the foam and resin, respectively.

The predicted microbuckling and elastic buckling strengths are shown in Figure 7.14 for each of the core densities tested, along with the actual measured values. It can be clearly seen that elastic buckling is likely to be operative at two lowest densities, but microbuckling governs the strength at all higher densities. It is noted that the microbuckling failures occurred near the nodes in the region of transition from the flat node to the straight truss. The braid is bent in this region, and therefore the fibers in this region are more highly misaligned than the unbent regions. Failure at this location is therefore consistent with the expectation that microbuckling would initiate within areas of the highest fiber misalignment ${ }^{64}$. 

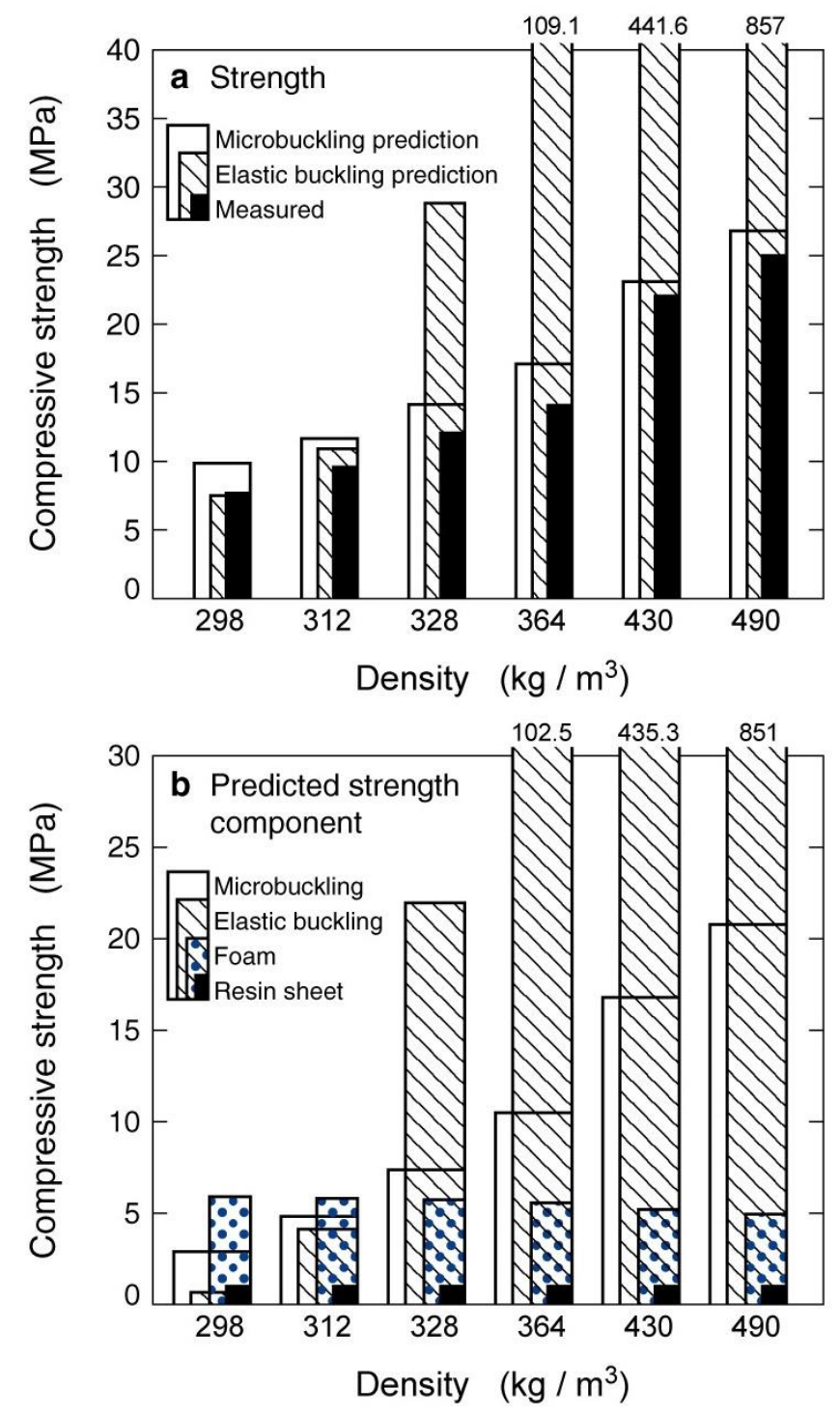

Figure 7.14: (a) The measured core compressive strength compared to micromechanical predictions, and (b) the components of the compressive strength prediction.

Figure 7.15 shows a plot of the predicted elastic buckling and microbuckling stresses versus the cores truss relative density. Also shown is the measured compressive strength of the CFRP pyramidal lattice obtained by subtracting the strength of the foam 
and resin sheet from the measured peak strength of the hybrid core. It can be seen that there is good agreement between the measured compressive strengths and the predictions. The small differences between the theoretical predictions and the measurements can be attributed to experimental uncertainty (one specimen tested per core density), as well as potential sample imperfections. It can also be seen that the relationship between the compressive microbuckling strength and the truss core fraction saturates as the relative density approaches $30 \%$. This is a result of the increasing truss mass fraction of the node, which does not support load as the truss diameter increases. Thus, the pyramidal structure becomes increasingly less efficient as the truss diameter increases, and $l$ becomes smaller. This effect is analogous to that reported for lattices made laminates ${ }^{4,34}$.

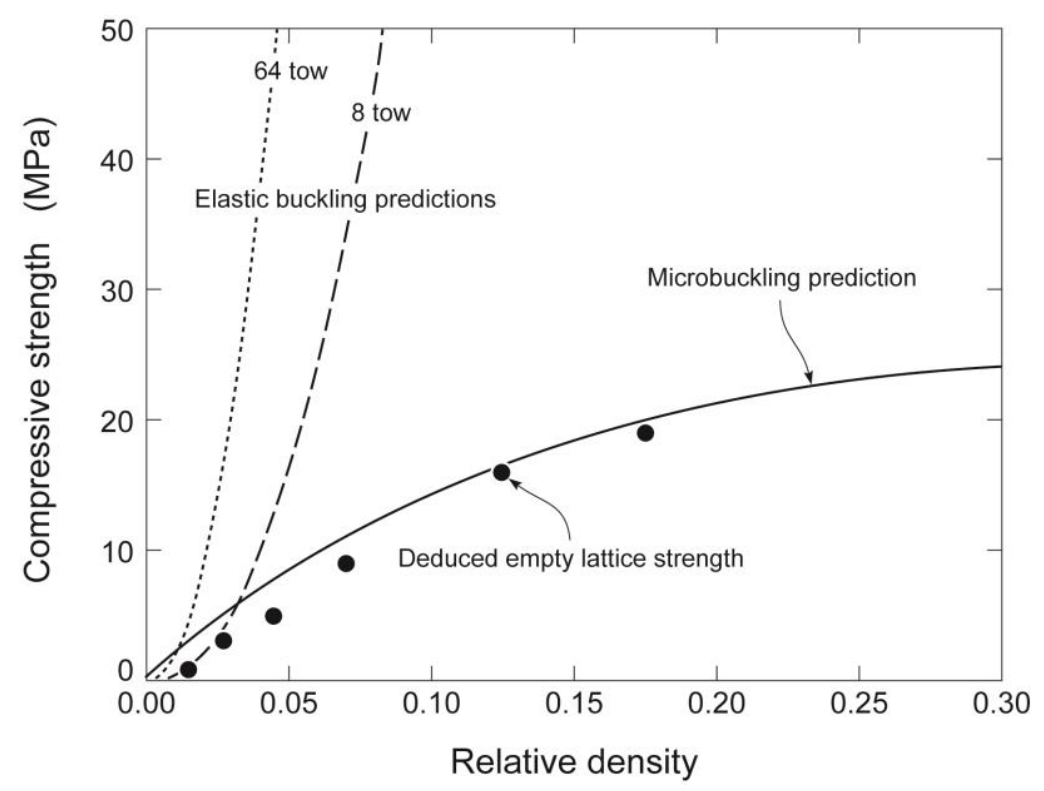

Figure 7.15: The elastic buckling and microbuckling predictions CFRP lattice for the compared to the deduced empty lattice strength (total core strength minus foam and resin sheet). 


\subsection{Energy absorption and densification}

The compressive strength and modulus of the hybrid CFRP lattices made from linear braids are plotted against density on modified Ashby charts in Figure 7.16. It is evident that the modulus of the hybrid linear braid structures, Figure 7.16a, significantly exceeds that of the hybrid braided net structures of similar core density, and can be attributed to the increased volume fraction of high specific stiffness CFRP trusses. It can be seen that the compressive strength of the linear braid hybrid structures is comparable to that of the hybrid structures made using a braided net, as well as other CFRP lattices and honeycombs. We note that the fraction of CFRP truss volume contained in the nodes (see Table 7.2) increases from $11.9 \%$ for the lowest density core to $35.4 \%$ for the highest density samples. Examination of Equations 7.8 and 7.9 indicate that similar lattice strengths could be obtained at lower densities, if the node volume could be eliminated. Efforts to achieve this were hampered by flattening of the braid near the face sheet, and severe weakening of the truss in that region. Some group ${ }^{65,66}$ have explored fabrication techniques which attempt to completely eliminate nodal volume by inserting CFRP pins into a foam between two face sheets. However this method might result in node to face sheet connectivity issues, and seems to work well for just slender struts.

The compressive stress versus strain curves for the hybrid specimens (Figure 7.9) show relatively long plateau stress regions with a plateau stress very close to the initial peak in strength. They are therefore likely to have promising uses for mechanical energy absorption during impact events. The energy absorbed per unit volume for the hybrid linear braid panels $W_{v}$, has been calculated in an identical manner to that reported by George, et al, by integrating the area under the stress strain curve up till the point of 
densification. The energy absorbed per unit mass, $W_{m}$, was found by dividing the energy absorbed per unit volume by the core density. The results are plotted in Figure 7.17. It can be seen that the energy absorbed per unit volume for the hybrid linear braid structures is slightly less than that of high foam mass fraction braided net structures, but substantially higher than CFRP honeycombs and lattices.
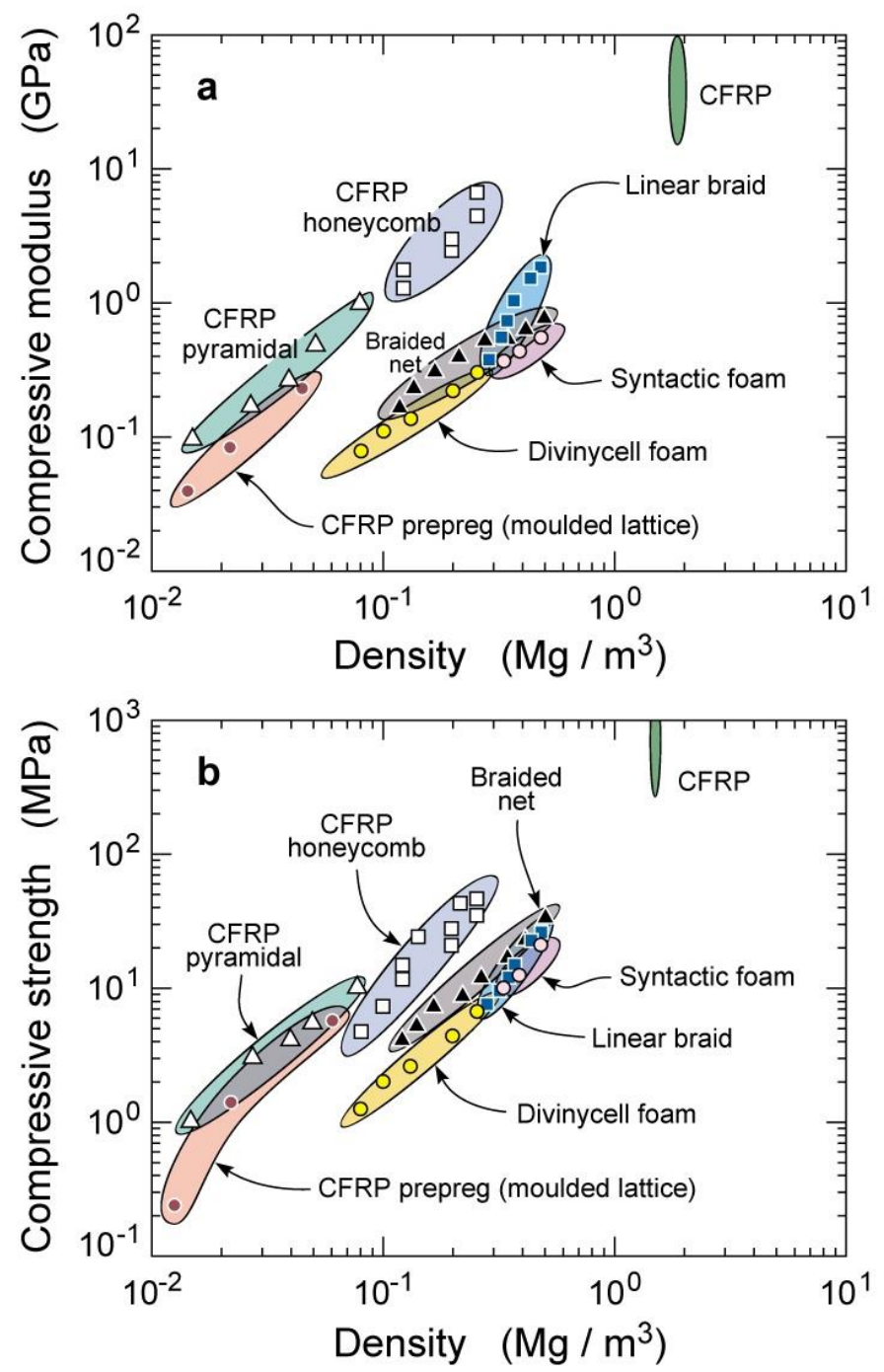

Figure 7.16: Material property chart showing (a) compressive strength, and (b) compressive modulus of CFRP honeycomb and pyramidal truss structures. The linear braid results of this study are also shown together with that of the foams. 

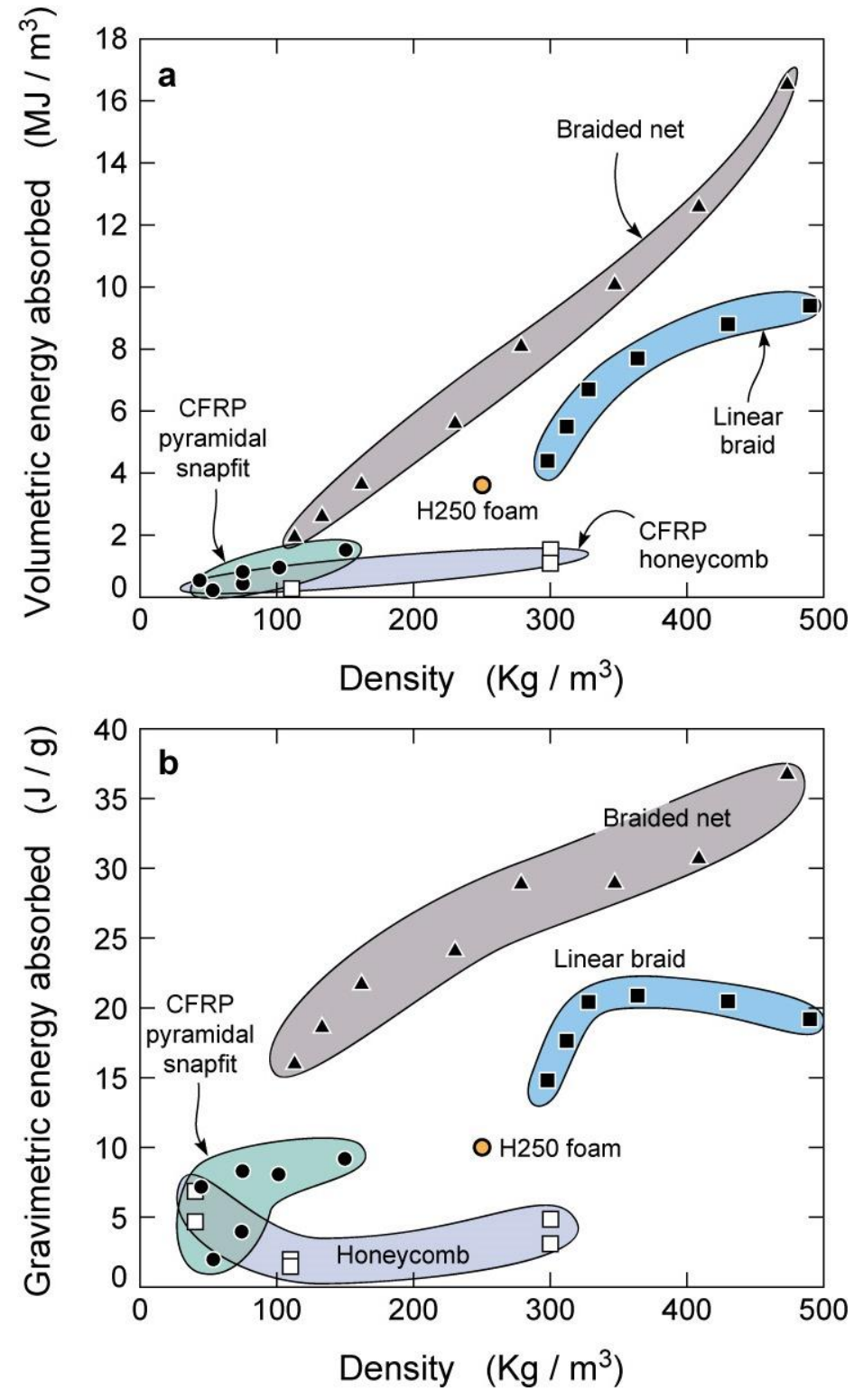

Figure 7.17: (a) The energy absorbed per unit volume and (b) energy absorbed per unit mass both plotted against density for low density cellular materials. The hybrid composites made using linear braids compare favorably with the other materials.

It can also be seen that the volumetric and gravimetric energy absorptions of the linear braid hybrid structures do not increase with hybrid core density as rapidly as the 
braided net structures. This is the result of saturation of the core strength and a decrease in densification strain, $\varepsilon_{d}$, as the truss volume fraction increases. It has been recently shown that the densification strain for a composite structure can be predicted based on the truss length, thickness, angle of inclination, and the bent fractional truss length ${ }^{67}$. The application of this principle to the structure investigated here is shown in Figure 7.18a. Analysis of the geometry indicates that the densification strain of the linear braid hybrid cores is given by:

$$
\varepsilon_{d}=\left(1-\frac{d_{1}}{l \sin \omega}-\frac{1-x}{\sin \omega} \sqrt{1-\left(\frac{\cos \omega-x}{1-x}\right)^{2}}\right)
$$

where $d_{l}, l$, and $\omega$ are the unit cell parameters described in Section 7.2.4. The bent fractional truss length, $x$ can be determined from XCT images of samples that have been compressed to densification (Table 7.2)

Figure 7.18 shows a plot of the predicted densification strain for each of the truss relative densities tested compared to the measured values (Table 7.2) It can be seen that there is very good agreement between the predicted and measured densification strain values. Equation 7.12 suggests that the densification strain could be increased by reducing the ratio of the truss diameter to the truss length $\left(d_{l} / l \sin \omega\right)$, and that lower relative density cores would have a higher densification strain. Given that the ideal energy absorber should have both high strength and a high densification strain, this would suggest that for energy absorption applications, the truss relative density within the hybrid should be kept relatively low. Equations 11 and 12 can be used to estimate the 
energy absorption capacity for a hybrid core for a given set of unit cell parameters, and would be very useful for materials design and analysis.
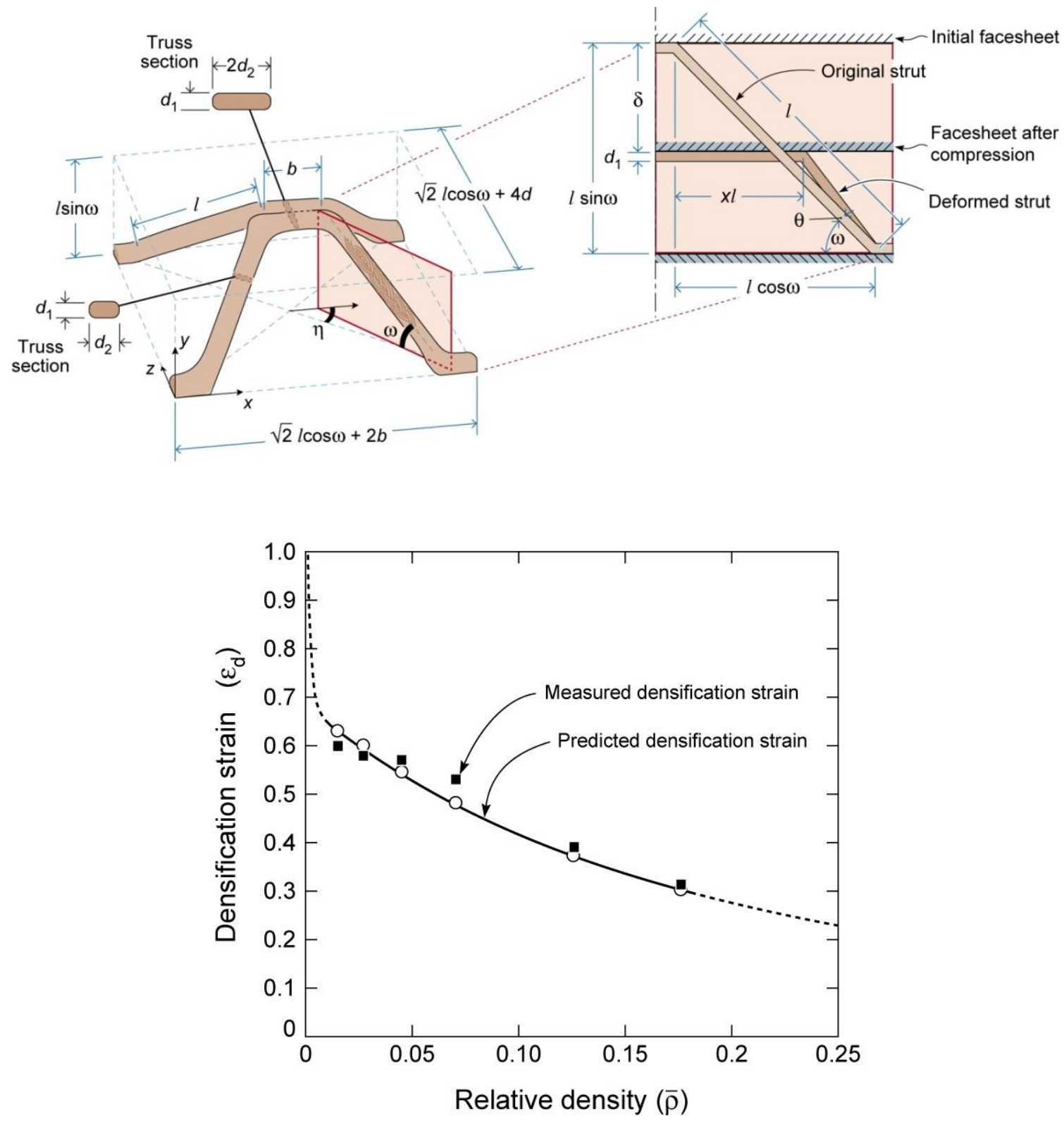

Figure 7.18: (a) Schematic of densification model used to predict densification strain (b) The predicted densification strain compared to the measured values for the truss relative densities tested. 


\section{Chapter 8}

\section{Hybrid CFRP Octet Lattice Structures}

The pyramidal (or related tetrahedral or kagome) lattice has a fixed relation between cell height and base. If lattice core sandwich panels with widely separated faces are used, the nodal connections between trusses and the faces would be widely separated, leading to significant stress concentration upon loading. This chapter explores the feasibility of fabricating hybrid CFRP/foam core sandwich structures with an octet truss topology that can have a cell size independent of face sheet separation. The CFRP trusses for the octet structure can be fabricated from linear carbon fiber tows, or pultruded carbon fiber composite rods which have a higher compressive strength. This chapter describes the fabrication and mechanical properties of both types of hybrid CFRP/foam octet lattice structures.

\subsection{Hybrid Design and Assembly}

\subsubsection{Design Concept}

The design of the composite structure explored here is shown schematically in Figure 8.1. The structure can be assembled in layers, using a polymer or syntactic foam as a space holder to form the lattice shape. The foam space holders can be machined with holes and grooves at specified spots such that upon assembly of the mold layers a complete octet structure is formed. Then carbon fiber tows, or pultruded carbon fiber rods can be inserted in the holes present to create the structure. The entire assembly can then 
be infiltrated with epoxy using a vacuum assisted resin transfer molding (VARTM) process.

\subsubsection{Foams}

To avoid resin infiltration of the foam, only closed cell foams can be used. Many types of closed cell foams could be used as the spaceholder for the octet structure. The foams used included the closed cell polymer Divinycell H80 and H250 foams, with densities of 80 and $250 \mathrm{kgm}^{-3}$, and a commercially available syntactic Trelleborg DS39 foam were investigated. In addition, as well as a syntactic foam made from hollow glass microspheres and an SC1 epoxy resin was also evaluated.

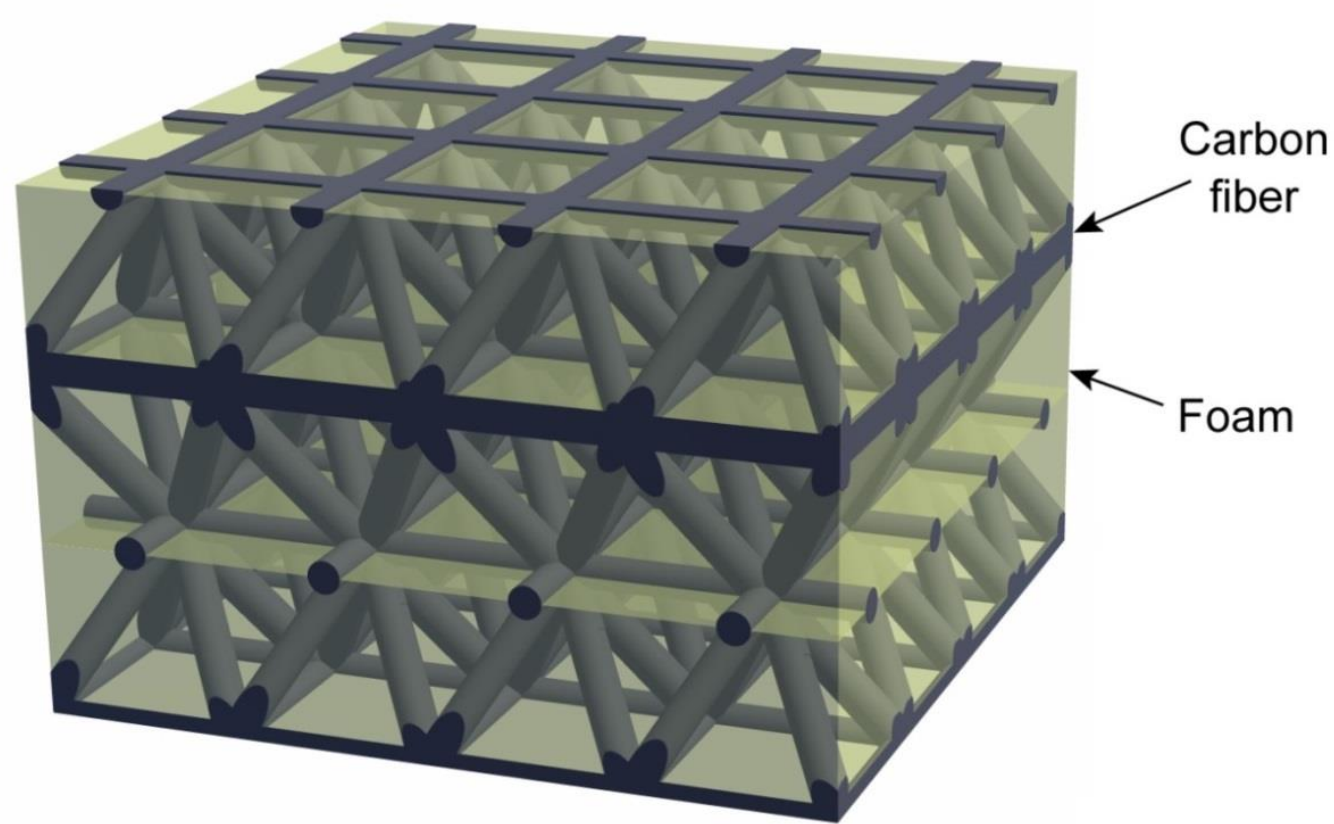

Figure 8.1: Schematic illustrating the design concept for the hybrid octet lattice 
The SC1 resin syntactic foam used in this study was created by mixing a high strength epoxy resin (SC1) with grade iM-30k hollow glass microspheres (3M Corporation, St Paul, MN). The iM-30k glass spheres were chosen because of their high crush strength and low density. The iM-30k glass spheres are made using soda lime borosilicate glass, and have an average diameter size of 18 microns, and a true density of $600 \mathrm{~kg} / \mathrm{m}^{3}$, and a bulk crush strength of 195MPa. The SC1 epoxy resin, and curing agent was obtained from Applied Poleramic (Benicia, CA). To identify the best volume fraction of hollow microspheres to use, predetermined quantities of the hollow glass microspheres and epoxy were measured out using calibrated measuring cylinders, and mixed together within a square aluminum mold, as schematically illustrated in Figure 8.2.

a Measure out glass beads and epoxy
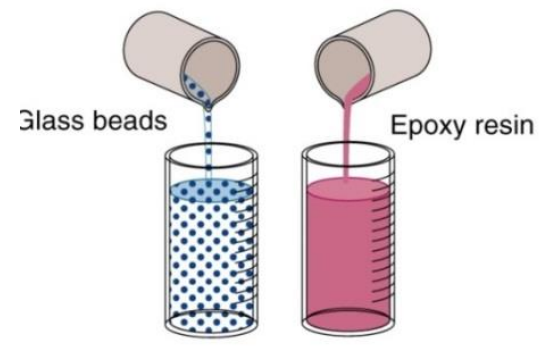

c Thoroughly mix and allow to solidify

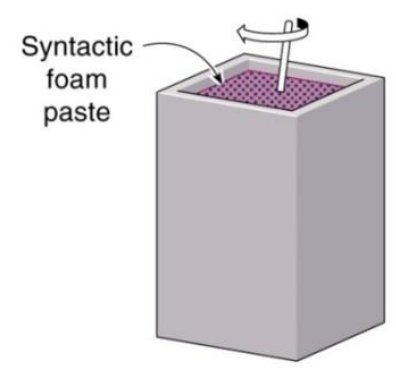

b Slowly mix small volumes while stirring

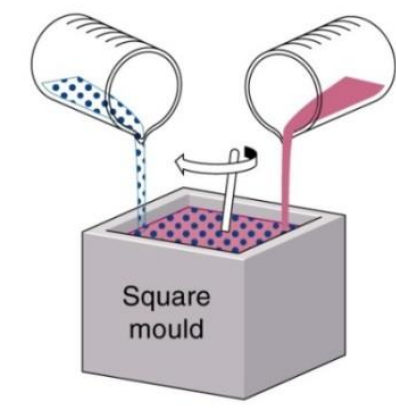

d Cure in furnace at $160^{\circ} \mathrm{F}$ for 4 hours
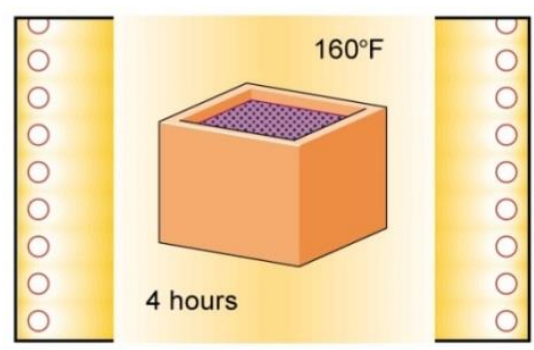

Figure 8.2: Schematic showing the fabrication procedure for the syntactic foams. 
The resin and the microspheres are thoroughly mixed together by mechanical stirring within the mold. The volume fraction of microspheres within the mix was varied from $21 \%$ to $65 \%$. The microspheres and epoxy form a viscous white paste. The mixture was allowed to solidify at room temperature, and then placed in a furnace at $71^{\circ} \mathrm{C}$ for 4 hours to complete the cure. After the curing of the syntactic foam mixture was completed, the solidified foam block was removed from the mold, and machined to into 40x40x20 mm blocks for compressive testing. The blocks were subsequently tested in compression at a strain rate of $2 \times 10^{-4} \mathrm{~s}^{-1}$ at room temperature $\left(23^{\circ} \mathrm{C}\right)$. Figure 8.3 shows compressive stress-strain curves for different volume fractions of microspheres. It can be seen from the stress strain cures that the toughness of the foam decreases as the volume fraction of glass microspheres increases. This is to be expected, given the fact that the resin has a higher toughness than the glass microspheres. The microstructure of $65 \%$ volume fraction syntactic foam is shown on the SEM image in Figure 8.4 below. This foam was selected for use in machining the molds due to its lower density, to ensure that the overall density of the finished hybrid composite would be as low as possible. 

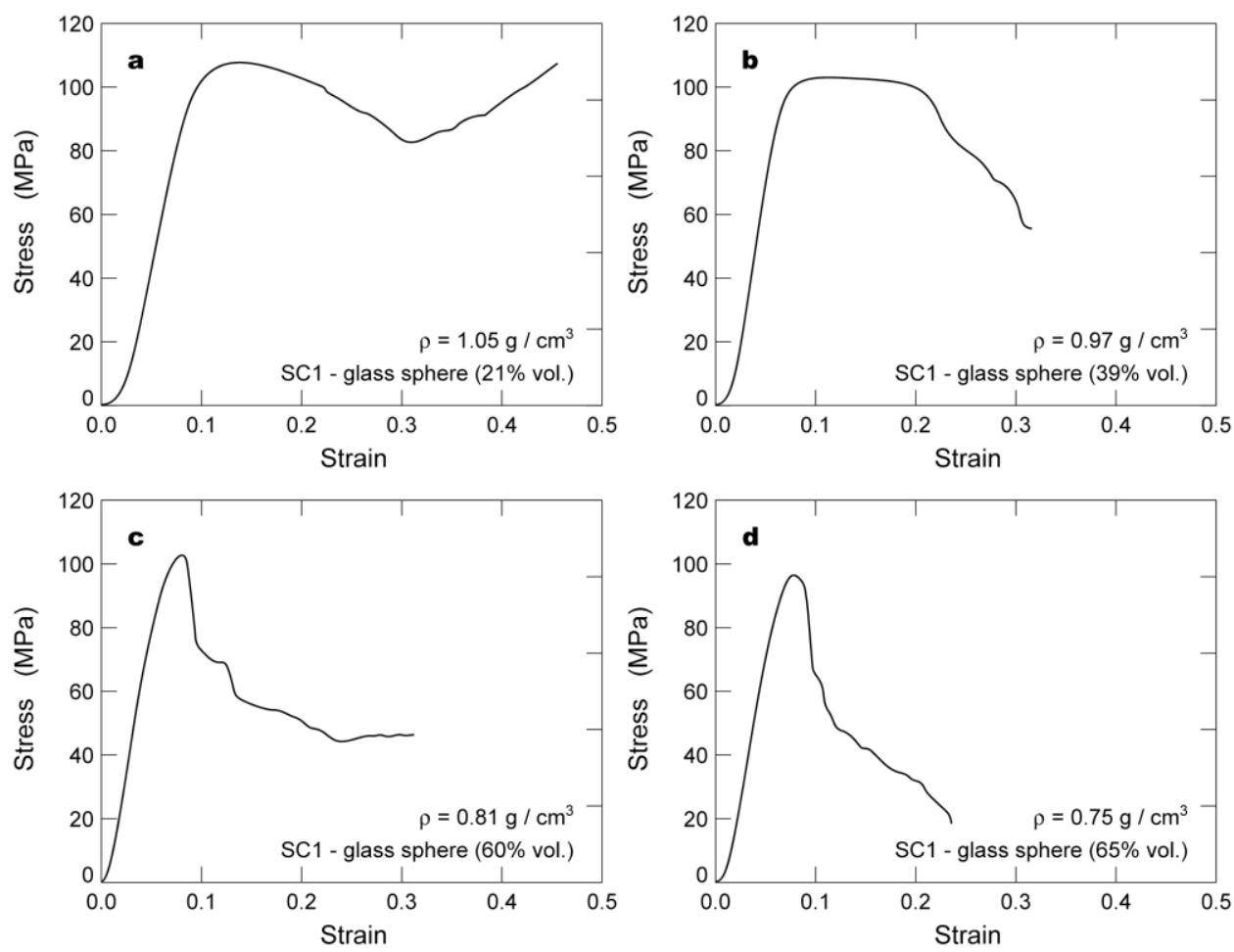

Figure 8.3: Compressive stress-strain curves for SC1 syntactic foams with microsphere volume fractions of (a) $21 \%$, (b) $39 \%$, (c) $60 \%$, and (d) $65 \%$ respectively.

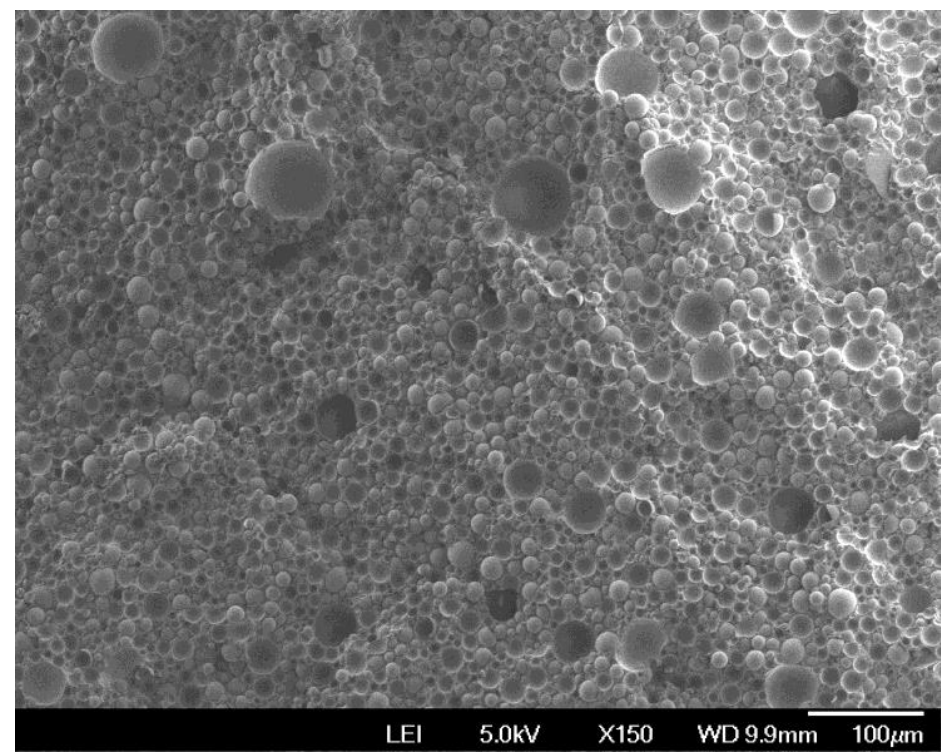

Figure 8.4: SEM image showing the microstructure of the $65 \%$ glass volume fraction SC1 syntactic foam. 


\subsubsection{Dry Panel Assembly}

The carbon fiber octet structures were assembled using two different techniques. The first technique (Figure 8.5) used carbon fiber tows to formulate the struts. It involved stacking 5 mold layers, and subsequently pulling carbon fiber tows into the holes within the mold using a mechanical needle. Four tows of $12 \mathrm{k}$ (12,000 fibers per tow) IM7 carbon fiber ${ }^{\text {iv }}$ were pulled into each of the holes. The design of the holes within the mold layers ensured that when the fiber tows interpenetrate each other at the nodes, with each subsequent bundle of tows interpenetrating the previous bundles already in place, until all four bundles of tows at each node are in place. Care was taken to ensure that all the holes within the mold structure are filled with the same number (4) of fiber tows. After this process was complete, the excess fiber on the outside of the mold structure is trimmed, and the panel is ready for resin infusion.

\footnotetext{
${ }^{\text {iv }}$ Manufactured by Hexcel Corporation, Stamford, CT
} 
a

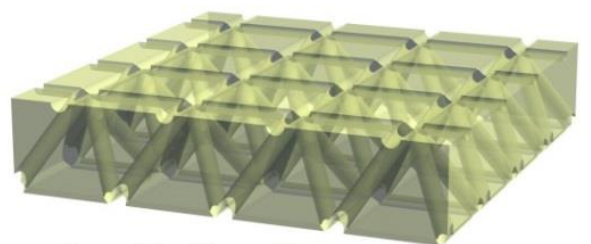

Pyramid void mould b

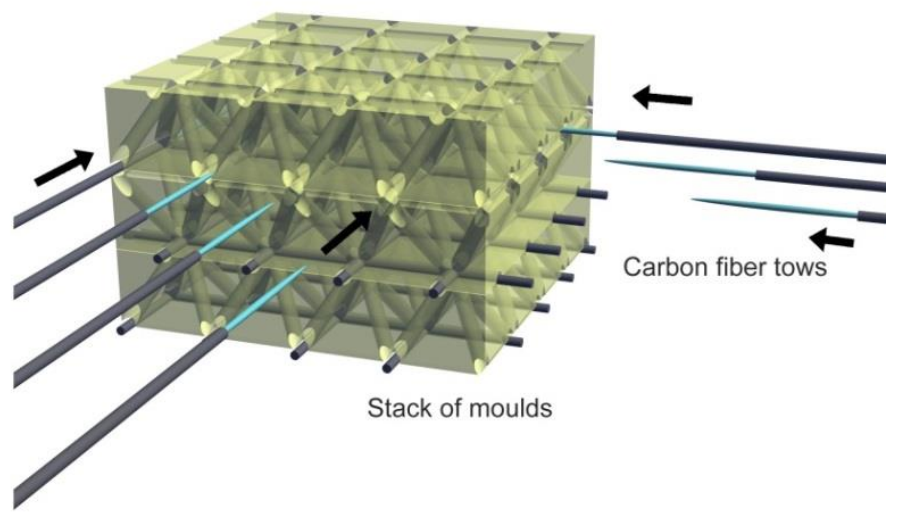

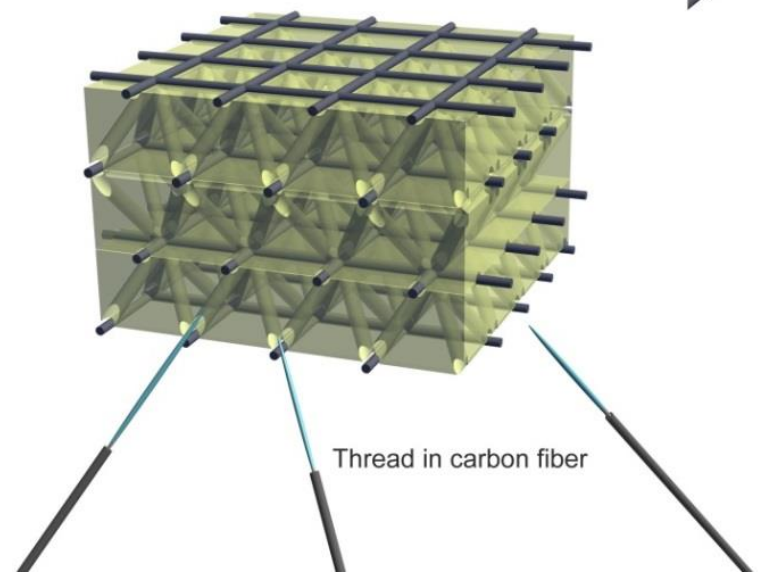

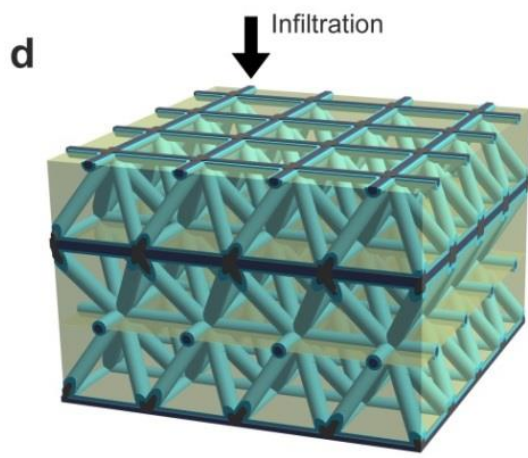

Figure 8.5: Schematic showing the assembly procedure for the hybrid octet lattice utilizing carbon fiber tows and a fiber threading method.

The second technique (Figure 8.6) utilized pultruded carbon fiber composite rods to fill the machined holes within the mold. This technique had the advantage of reducing the fiber waviness within the trusses of the structure, given that the pultruded rods have a high degree of fiber alignment. This technique also had the advantage of substantially reducing the time required to assemble each sample. The pultruded rods are inserted into the holes for each foam layer, and the layers subsequently stacked. The carbon fiber pultruded rods (type AE001180, Graphite Store Inc., Buffalo Grove, IL) used had a 
nominal diameter of $2 \mathrm{~mm}$, and were cut into $10 \mathrm{~mm}$ long lengths for insertion into the foam mold. The rods were inserted only on the $45^{\circ}$ angled holes within the foam plate, while 4 tows of $12 \mathrm{k}$ IM7 carbon fiber were placed in the horizontal holes. The carbon fiber rods in the $45^{\circ}$ angled holes slightly interpenetrated the horizontal tows at the nodes, and the nodes were subsequently held in place only by epoxy.

a Pyramid void mould

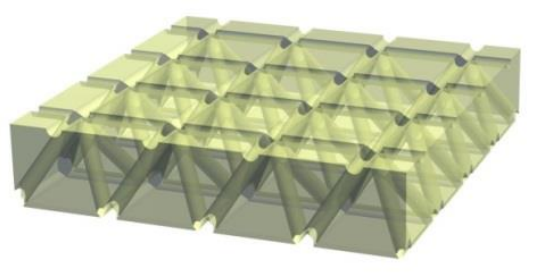

C

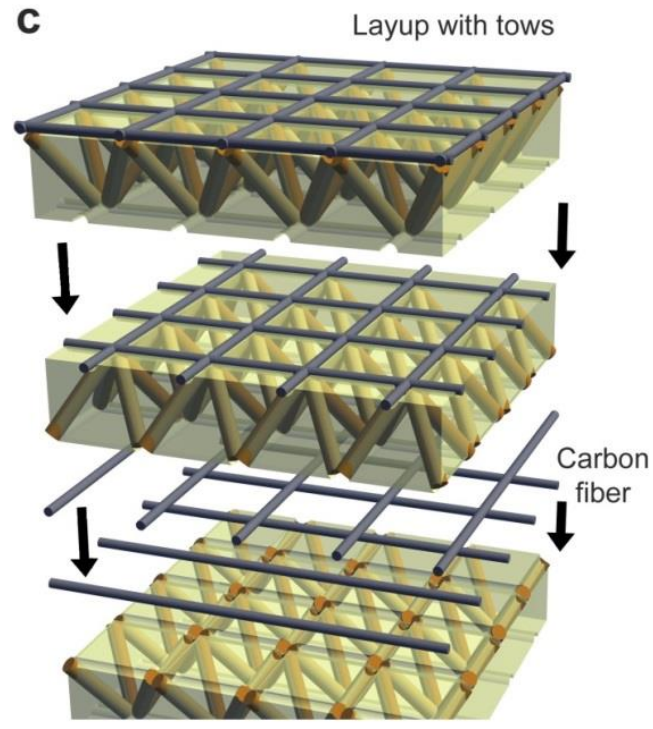

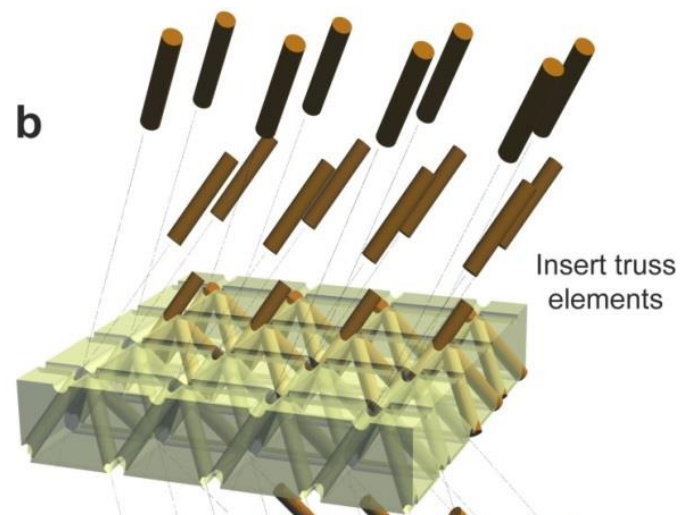

b

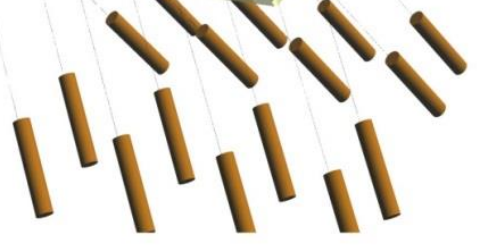

d

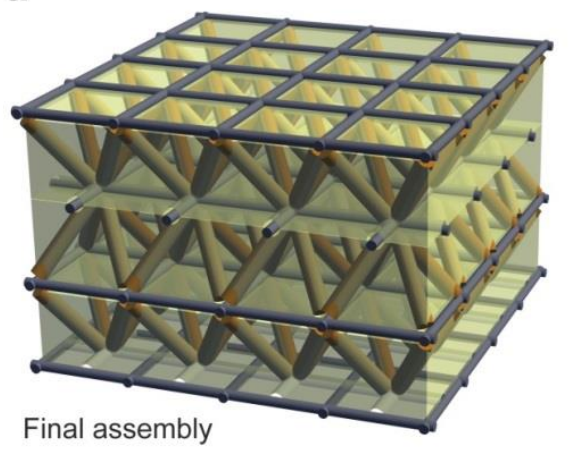

Figure 8.6: Schematic showing the assembly procedure for the hybrid octet lattice utilizing pultruded rods. 


\subsubsection{Resin Infusion Process}

The assembled specimens was then infused using SC1A epoxy resin with SC1B curing agent. This resin was selected for infiltration because of its low viscosity, which allowed for smooth resin flow and complete infiltration of the part, its moderately high strength, and to enable comparison with previously made specimens. A vacuum assisted resin transfer molding (VaRTM) process is used to infuse the assembled dry panel. The setup for the infusion process is illustrated in Figure 8.7. The infusion and cure cycle were performed in an autoclave, which enabled control of the temperature, pressure, and vacuum within the assembly. The VARTM process used is identical to the process used to fabricate the linear braid and braided net hybrid structures. After the infusion and cure cycle is complete, the completed panel is removed, and the excess material trimmed using a wet saw. A picture of the composites made using $\mathrm{H} 80$ and $\mathrm{H} 250$ foam, and carbon fiber tows, is shown in Figure 8.8.

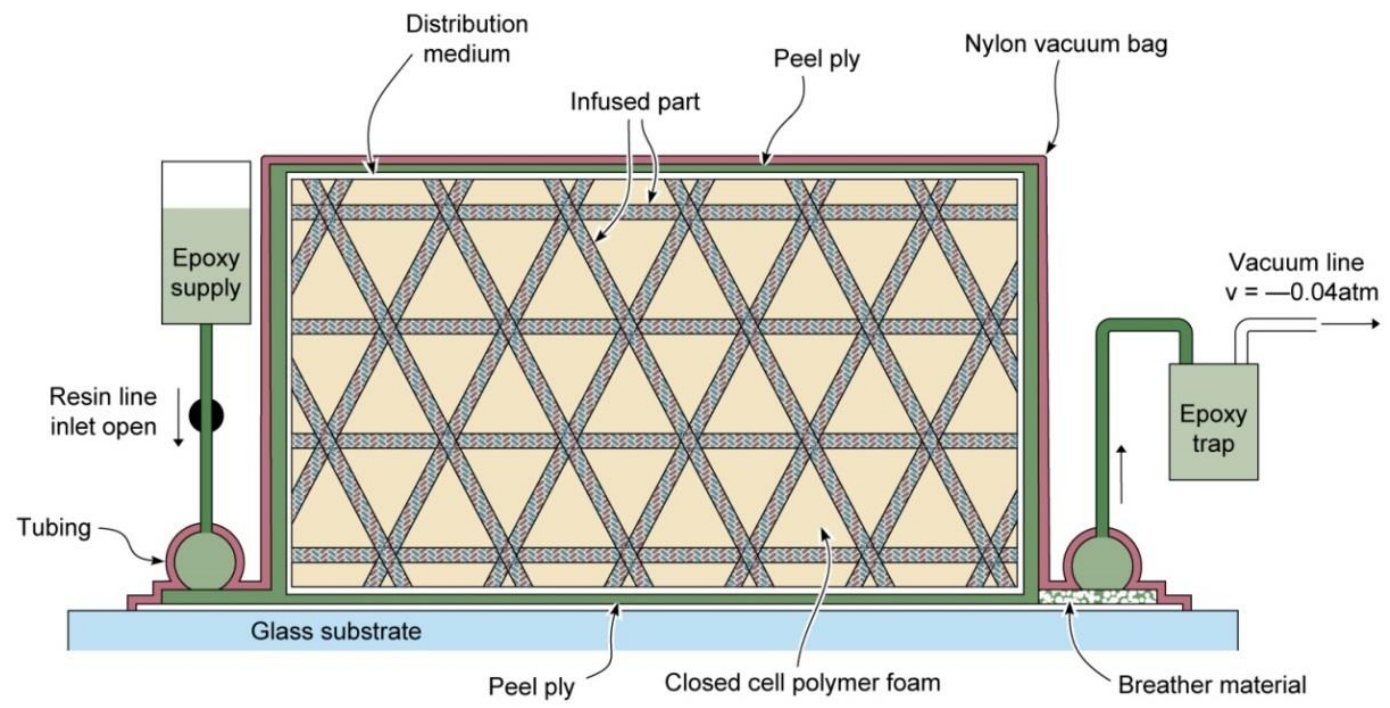

Figure 8.7: The infiltration setup for the octet hybrid structures. 


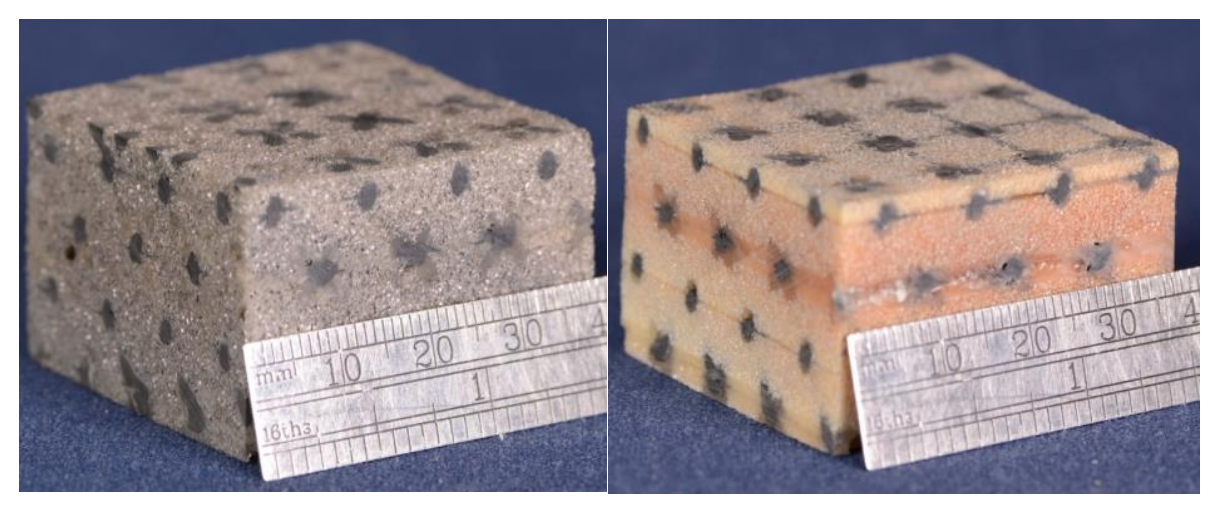

Figure 8.8: Photographs showing finished octet hybrid specimens made using H80 and H250 divinycell polymer foams and linear tow trusses.

\subsubsection{Unit Cell Geometry}

The octet truss hybrid specimens unit cell is shown in Figure 8.9. The dimensions of the unit cell are as follows: The diameter of the trusses, $d$, is $2 \mathrm{~mm}$ in all cases. The length of the truss ligaments $l$ (identical to the node to node spacing), is $10 \mathrm{~mm}$. The angle of inclination of the trusses, $\omega$, is $45^{\circ}$. The relative density $\bar{\rho}$ of the lattice, as shown in Chapter 2, is found by calculating the ratio of the truss volume to the volume of the unit cell, and can be written as:

$$
\bar{\rho}=6 \sqrt{2} \pi\left(\frac{r}{l}\right)^{2}
$$

Where $r$ is the radius of the trusses. The relative density of the unit cell, given the dimensions, is $26.4 \%$. The total density of the hybrid octet core $\rho$ can be found by adding the density of the trusses to the density of the surrounding foam material. It is assumed that the volume within the unit cell that is not occupied by trusses is occupied by foam. The total density of the hybrid core, can then be written as: 


$$
\rho=\left(\bar{\rho} \rho_{t}\right)+(1-\bar{\rho}) \rho_{f}
$$

where $\rho_{t}$ is the density of the truss material $\left(1450 \mathrm{kgm}^{-3}\right.$ for the infused tows and 1550 $\mathrm{kgm}^{-3}$ for the pultruded rods), and $\rho_{f}$ is the density of the surrounding foam. Each octet hybrid specimen was four unit cells in width and length, and 1.5 unit cells in height.

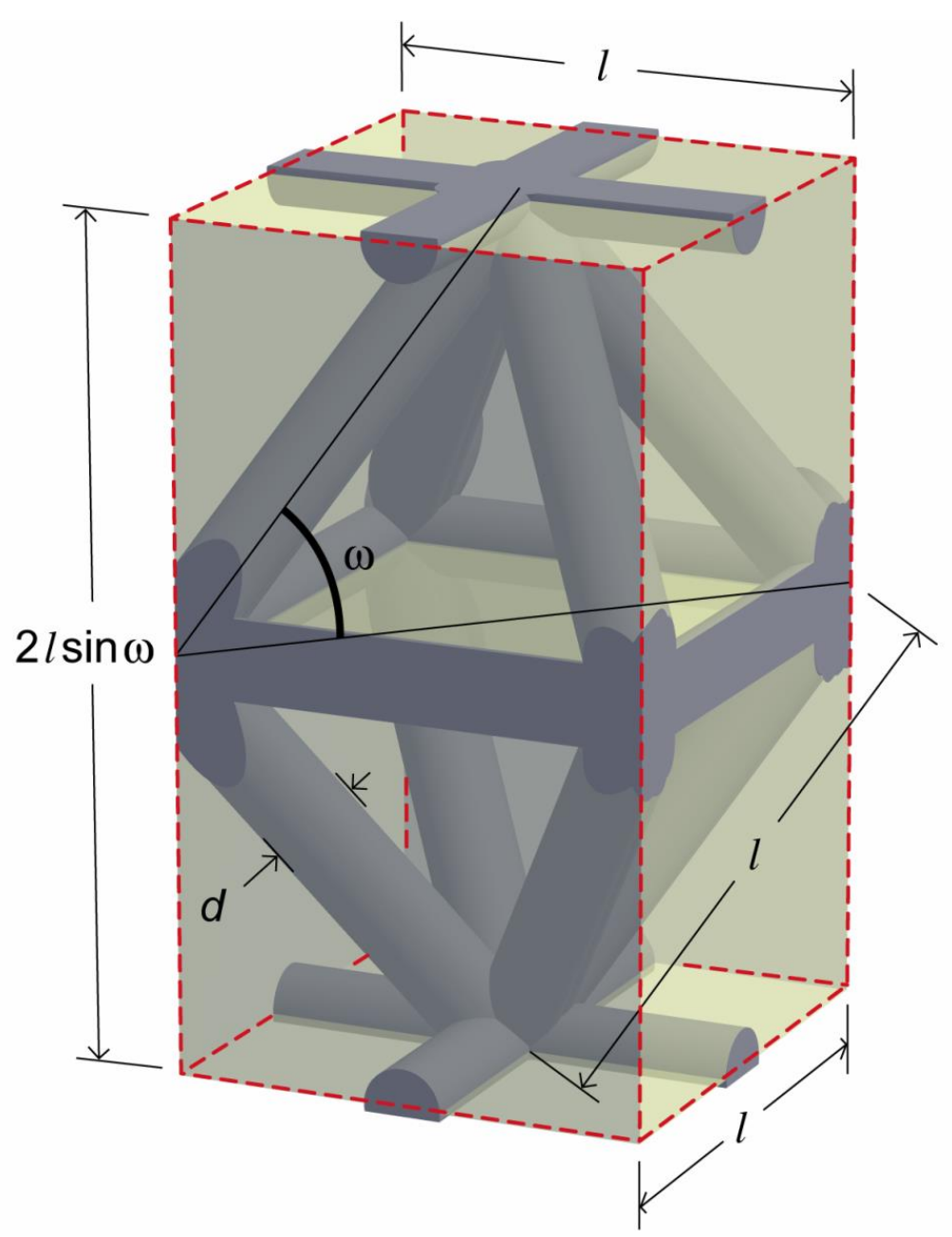

Figure 8.9: Unit cell of the octet truss/foam hybrid. 


\subsection{Truss Properties}

\subsubsection{Compression}

The compressive moduli and strength of the infused linear tow and the pultruded rod trusses have been measured. For the infused linear tow trusses, samples were prepared by infiltrating SC1 epoxy resin through a glass tube of diameter $d=2 \mathrm{~mm}$ contained four 12k IM7 tows. The resin was subsequently cured, and glass tubing was removed, leaving behind a cylindrical truss of diameter $2 \mathrm{~mm}$, that was then cut into $20 \mathrm{~mm}$ lengths and epoxy coated on both ends to prevent brooming failure. The volume fraction of the cured specimen was 0.41 , the same as in the struts of the octet hybrid. The pultruded rod specimens were prepared by cutting $20 \mathrm{~mm}$ lengths off the as-received rods, and subsequently coating the ends with epoxy, and placing in slots in aluminum tabs. The tabbed specimens were then compressed at a rate of $2 \times 10^{-4} \mathrm{~s}^{-1}$. The compressive strengths and moduli of both the infused linear tows and the pultruded rods are given in Table 1. Stress strain curves the compressive test for both truss materials is shown in Figures 8.10a and 8.10c. Figure 8.11 shows an XCT image slice of the mid-plane of a failed linear tow truss. It can be seen that the truss fails by microbuckling of the fiber tows. This involved a double "kink" of fibers on an inclined plane near the center of the sample, and buckling of the surrounding composite, leading to its brittle failure.

Delamination and brooming are not operative failure mechanisms, as the specimen was designed to avoid both, with tabbing on both ends. 


\subsubsection{Tension}

The tensile strength of the truss was determined in accordance with the ASTM D3039 standard for tensile tests. The linear tow samples were prepared in the same manner as the samples used for compressive testing. 70mm long specimens were cut from both the cured linear tows and the pultruded rods for tensile testing. The samples were securely clamped using a v-notched grip, with a specimen gauge length of $20 \mathrm{~mm}$ between the grips. The samples were then tested at ambient temperature at a strain rate of $2 \times 10^{-4} \mathrm{~s}^{-1}$. Five samples were tested for both truss materials, and the average tensile strength and moduli is summarized in Table 8.1. Also indicated are the range of measurements. Figures $8.10 \mathrm{~b}$ and $8.10 \mathrm{~d}$ show a typical stress strain curve in tension for each of these materials. The specimens fail by tensile fracture and pullout of the fibers. 

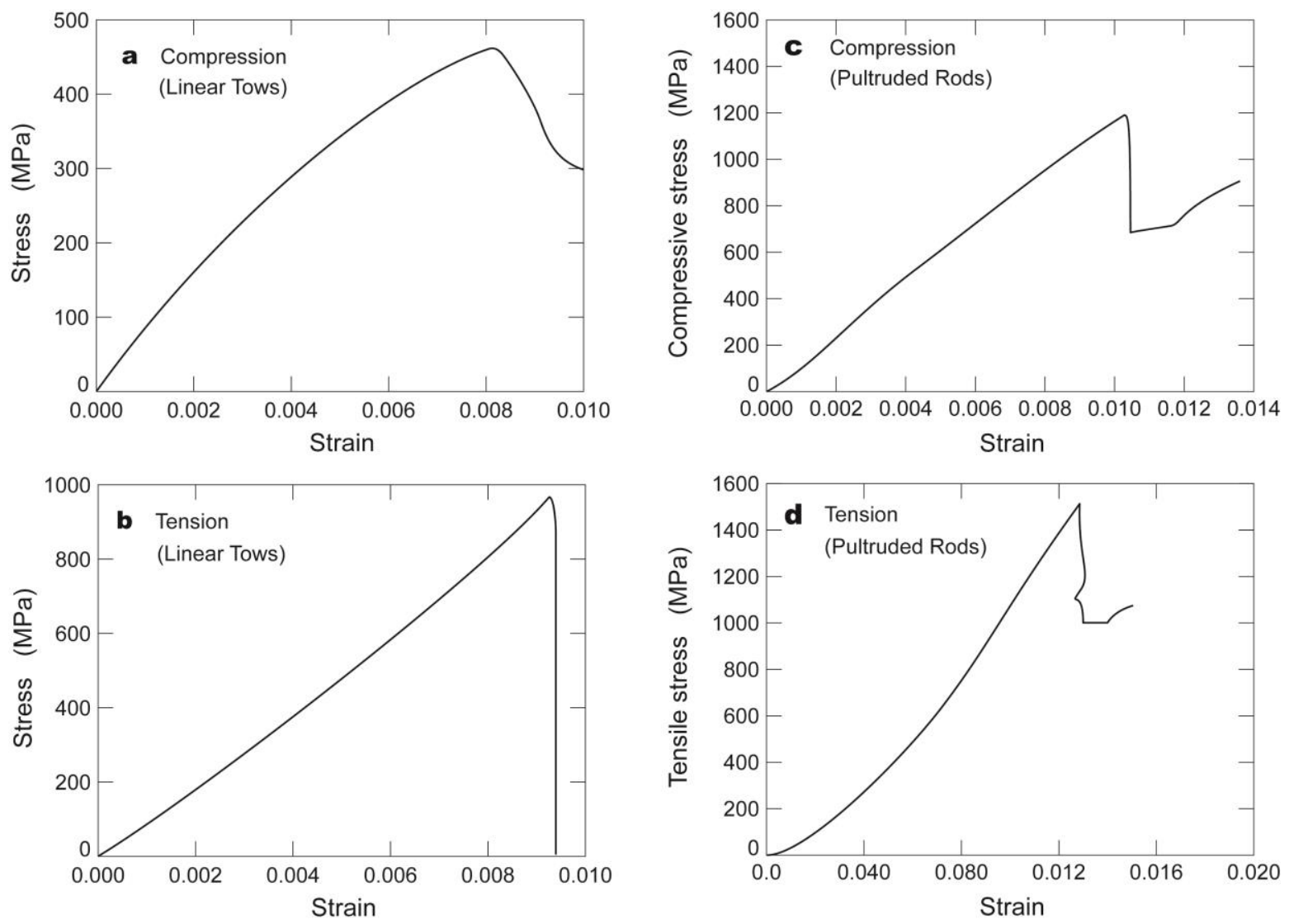

Figure 8.10: Stress-strain curves for the linear tow and pultruded rod trusses.

Table 8.1 CFRP Truss Properties

\begin{tabular}{|l|r|r|}
\hline & \multicolumn{1}{|l|}{ Linear Tows } & \multicolumn{1}{l|}{ Pultruded Rod } \\
\hline Density $\left(\mathrm{kg} / \mathrm{m}^{3}\right)$ & 1400 & 1490 \\
\hline Compressive Strength $(\mathrm{MPa})$ & $470 \pm 35$ & $1100 \pm 51$ \\
\hline Compressive Modulus $(\mathrm{GPa})$ & $85 \pm 4$ & $110 \pm 5$ \\
\hline Tensile Strength $(\mathrm{MPa})$ & $970 \pm 40$ & $1450 \pm 55$ \\
\hline Tensile Modulus $(\mathrm{GPa})$ & $95 \pm 7$ & $117 \pm 9$ \\
\hline
\end{tabular}




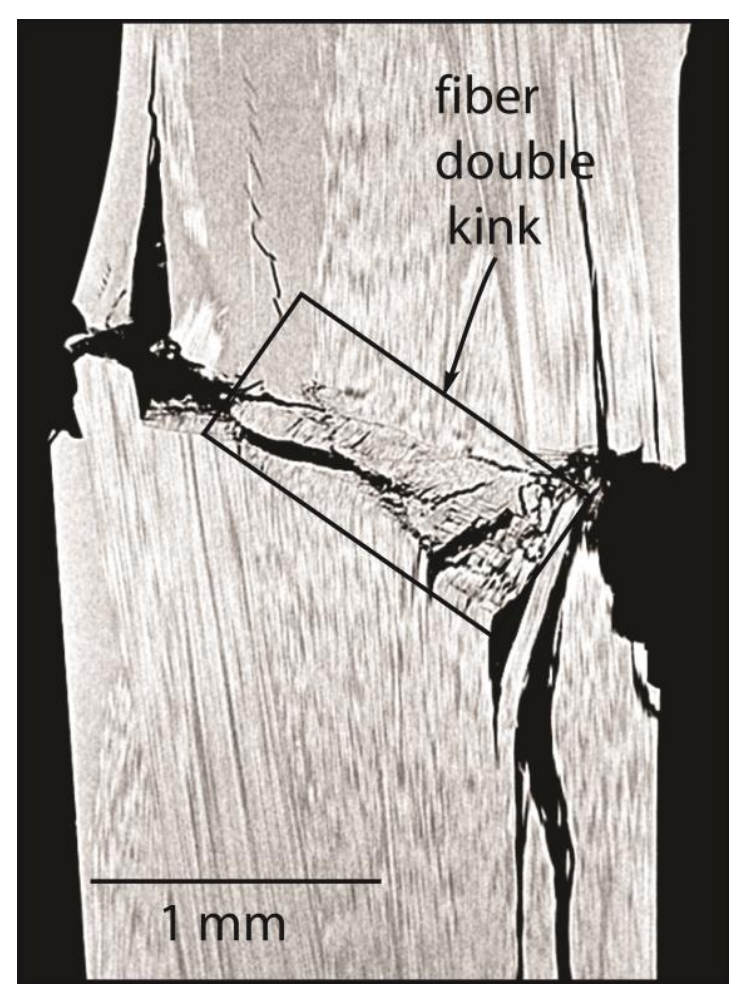

Figure 8.11: XCT image slice showing mid-plane of failed linear tow truss. It can be seen that the truss fails by microbuckling of the fiber tows.

\subsection{Hybrid Octet Lattice Response}

The compressive response of the panels was measured (in the direction perpendicular to the in-plane tows, such that the pyramidal struts are activated) using a screw driven universal testing machine (Model 4208, Instron Corporation, Canton, MA) with a $300 \mathrm{kN}$ load cell, in accordance with ASTM C365, the standard test method for flatwise compressive properties of sandwich cores, identical to the procedure used in preceding chapters. Retroreflective tabs were attached to the load platens on the top and bottom of the specimen, and a laser extensometer was used to record the displacement. 
The samples were tested at a nominal strain rate of $2 \times 10^{-4} \mathrm{~s}^{-1}$, and at ambient room temperature $\left(23^{\circ} \mathrm{C}\right)$. The elastic modulus for each specimen was measured by unloading within the nominally elastic region of the stress-strain curve.

The compressive stress strain response for the hybrid octet structure made using linear tows in Figure 8.12. Figures 8.12a-d shows the responses for the H80 foam, H250 foam, Trelleborg syntactic foam, and SC1 syntactic foam respectively. The stress strain curves were all initially linear, and yielding at a peak strength, followed by a drop in strength and a stress plateau. It can also be seen that the peak stress measured increases as the total density of the octet core increases. The stress strain curve for the octet structure made using pultruded rods and Trelleborg syntactic foam is shown in Figure 8.13. The measured compressive properties are summarized in Table 8.2.

Figure 8.14 shows reconstructed XCT images of an H250 foam/CFRP truss hybrid, in an unstrained state, and after loading to approximately $10 \%$ strain (one layer of cells is shown for clarity). The connectivity of the trusses within the specimen can be clearly seen. The failed trusses within the specimen are shown in black circles, and as indicated earlier, the linear tows fail by microbuckling. 

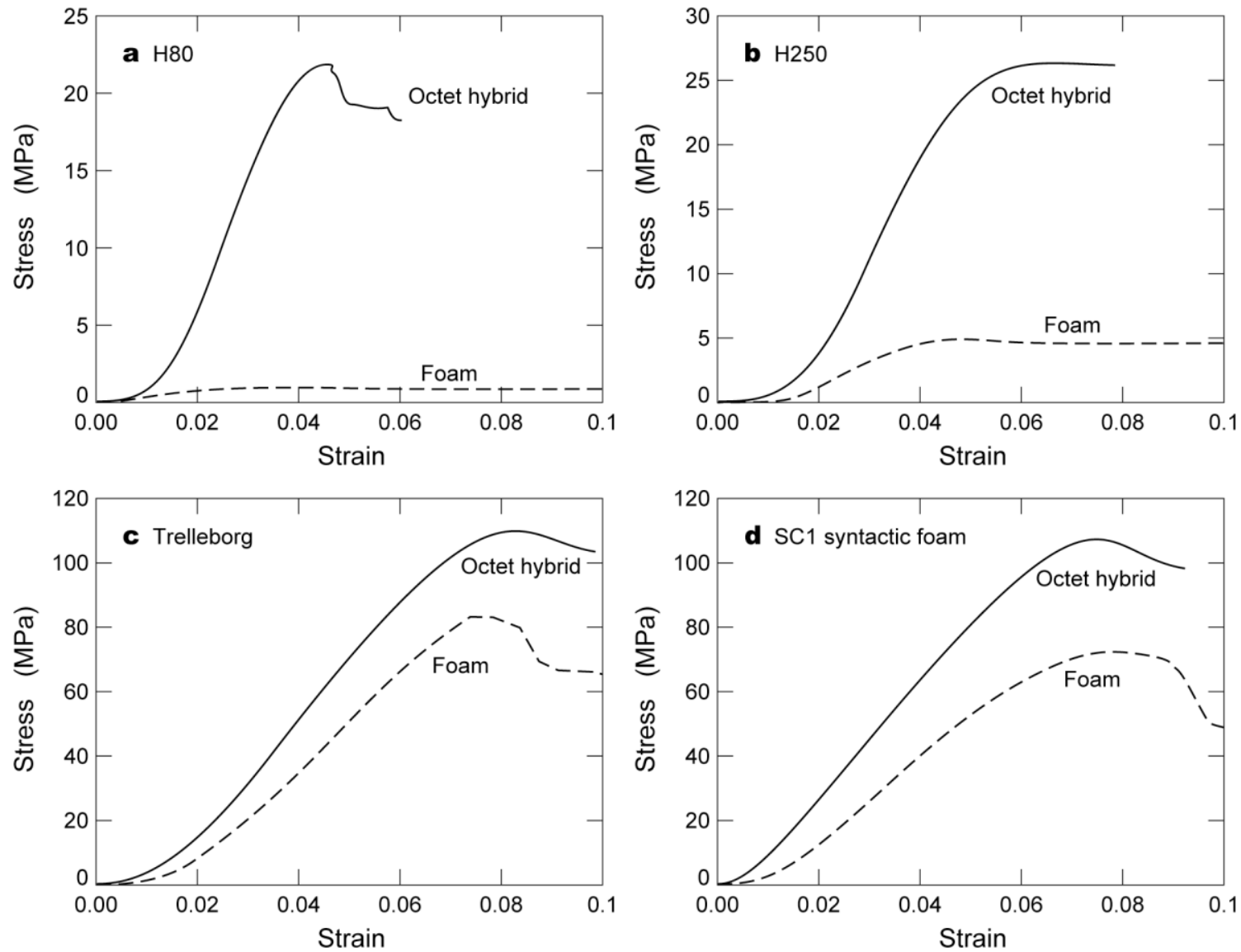

Figure 8.12: Compressive stress-strain responses for the hybrid octet lattices. Also shown are the stress-strain curves for the foams used, scaled by volume fraction within the hybrid. 
Table 8.2 Hybrid CFRP octet properties

\begin{tabular}{|l|r|r|r|r|r|}
\hline Specimen & \multicolumn{1}{|l|}{ H80 } & \multicolumn{1}{l|}{ H250 } & SC1 & Trelleborg & $\begin{array}{l}\text { Trelleborg } \\
\text { (Pultruded) }\end{array}$ \\
\hline Foam Density $\left(\mathrm{kg} / \mathrm{m}^{3}\right)$ & 80 & 250 & 750 & 650 & 650 \\
\hline $\begin{array}{l}\text { Foam Compressive } \\
\text { Strength (MPa) }\end{array}$ & 1.4 & 6 & 100 & 110 & 110 \\
\hline Truss relative density $\bar{\rho}$ & 26.4 & 26.4 & 26.4 & 26.4 & 26.4 \\
\hline Core Density $\left(\mathrm{kg} / \mathrm{m}^{3}\right)$ & 436 & 562 & 932 & 858 & 871 \\
\hline Truss used & 4 tows & 4 tows & 4 tows & 4 tows & $\begin{array}{r}\text { Pultruded } \\
\text { rod }\end{array}$ \\
\hline $\begin{array}{l}\text { Compressive Strength } \\
\text { (MPa) }\end{array}$ & 22 & 27 & 104 & 110 & 125 \\
\hline $\begin{array}{l}\text { Compressive Modulus } \\
\text { (MPa) }\end{array}$ & 640 & 750 & 1670 & 1714 & 2100 \\
\hline
\end{tabular}

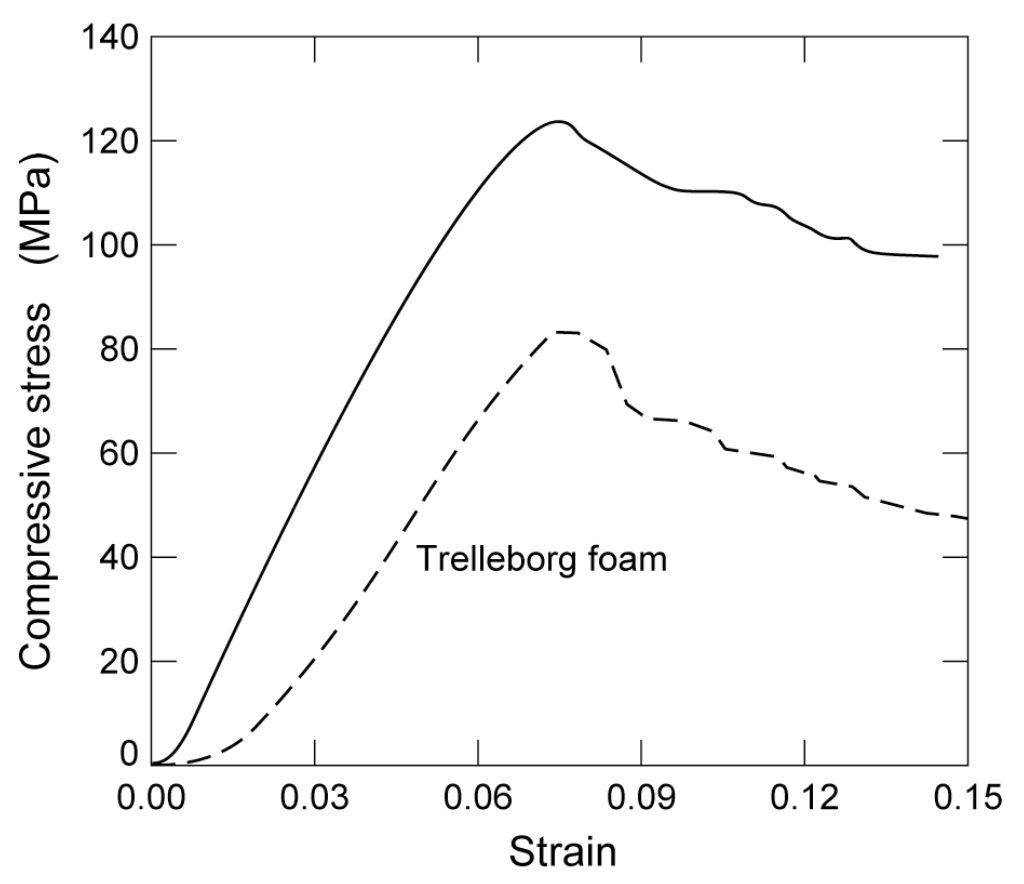

Figure 8.13: Stress-strain curve for hybrid octet specimen made using pultruded rod trusses. Also shown are the stress-strain curves for the foam used, scaled by its volume fraction within the hybrid. 


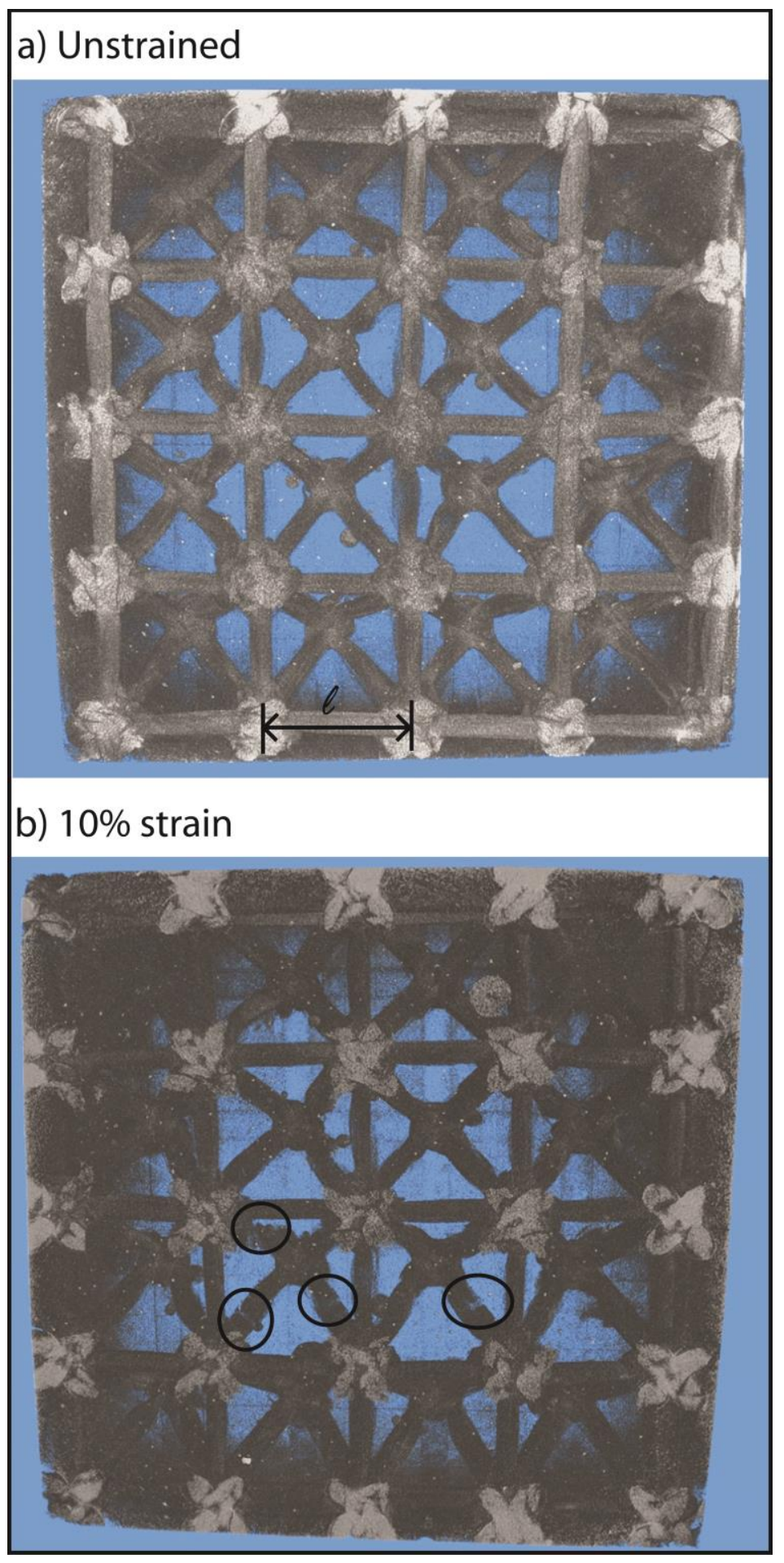

Figure 8.14: Reconstructed XCT images of a SC1 syntactic foam/ CFRP linear truss octet hybrid, showing one layer of cells a) as fabricated, and b) after loading to $10 \%$ strain. The failed trusses are shown in black circles. 


\subsection{Mechanical Behavior}

\subsubsection{Edge Effects}

It is noted that when the hybrid octet lattice is placed in through thickness compression, there are significant edge effects, whereby the trusses at the edges of the sample are not in contact with the load platens and therefore do not support any load. This effect was first discovered for diamond lattices ${ }^{61}$, and the knockdown effect on the compressive modulus and strength have been extensively analyzed. These studies have shown that this reduction in strength and modulus is related to the aspect ratio (length divided by height) of the specimen, as well as the truss inclination angle, and can be expressed as a knockdown parameter $K$, as shown below:

$$
K=1-\left(\frac{1}{A} \tan \omega\right)
$$

where $A$ is the specimen aspect ratio, defined as the length of the sample, $L$, divided by the height of the sample $H$; and $\omega$ is the angle of inclination of the trusses. This knockdown factor is used to obtain a lower bound estimate when predicting the strength and moduli of these lattices. For the hybrid octet lattices described in this chapter, we can estimate a knockdown factor by counting the number of unsupported inclined trusses, and dividing that by the total number of inclined trusses within the specimen. It is assumed that the tows that pass through the inclined holes are counted as single trusses, ignoring the nodes (i.e, the assumption is that the nodes provide no support). Using this method, only 16 of 80 trusses are fully supported, and $\mathrm{K} \approx 0.2$ for the hybrid octet specimens, which is a very significant effect. 


\subsubsection{Elastic Properties}

Deshpande and Fleck ${ }^{27}$ have extensively analyzed the micromechanical behavior of octet lattice structures, and their analysis is applied here. They have shown that for a through thickness compressive force on the octet lattice, the elastic modulus of the lattice is dependent on the modulus of the parent truss material, $E_{s}$, as well as the relative density of the lattice $\bar{\rho}$, and an upper bound estimate for the elastic modulus can be expressed as:

$$
E_{L}=\left(\frac{\bar{\rho}}{5}\right) E_{s}
$$

An upper bound estimate for the Young's modulus for the hybrid octet structure $E_{\text {core }}$ can be determined using the rule of mixtures, and accounting for the foam, and can be expressed as shown in the equation below:

$$
E_{\text {coreH }}=(1-\bar{\rho}) E_{\text {foam }}+E_{L}
$$

where $E_{\text {foam }}$ is the elastic modulus of the foam. A more reasonable estimate for the elastic modulus of the hybrid cores can be obtained by multiplying the lattice modulus contribution by the knockdown factor $\mathrm{K}$, as shown below:

$$
E_{\text {coreL }}=(1-\bar{\rho}) E_{\text {foam }}+K E_{L}
$$

The measured modulus of the hybrid octet structure is compared to the two predictions for different foams in Figure 8.15a. The measured values lie between the estimates, 
suggesting that the edge effects do significantly reduce the compressive modulus of the hybrid octet structure.

\subsubsection{Collapse Strength}

When an out of plane compressive force is applied to the octet lattice material, the lattice collapses either by plastic microbuckling of the constituent struts, or, in the case where the struts are slender enough, elastic buckling. The hybrid octet specimens are in the geometric regime $(d=2 \mathrm{~mm}, l=10 \mathrm{~mm})$ where elastic buckling is not operative.

Deshpande and Fleck ${ }^{27}$ have shown that for an octet lattice with these unit cell dimensions ( $d=2 \mathrm{~mm}, l=10 \mathrm{~mm})$, the compressive strength of the lattice is related to the compressive strength of the patent material as shown below:

$$
\sigma_{\text {lattice }}=0.085 \sigma_{p}
$$

where $\sigma_{p}$ is the compressive strength of the parent material. For the CFRP hybrid lattice, this strength is equivalent to the microbuckling strength of the linear tow and pultruded rod trusses, as measured in Section 8.3. An upper bound expression could then be written for the compressive strength of the hybrid octet lattice $\sigma_{\text {core }}$ using the rule of mixtures, as shown below:

$$
\sigma_{\text {core }}=(1-\bar{\rho}) \sigma_{\text {foam }}+\sigma_{\text {lattice }}
$$

where $\sigma_{\text {foam }}$ is the compressive strength of the foam. Similarly, a more realistic estimate can be obtained by incorporating the knockdown factor, as shown below: 


$$
\sigma_{\text {core }}=(1-\bar{\rho}) \sigma_{\text {foam }}+K \sigma_{\text {lattice }}
$$

The predictions for the compressive strength of the octet hybrid structures plotted along with the measured values in Figure $8.15 \mathrm{~b}$. It can be seen that measure compressive strength of the hybrid cores lie between the bounds indicated by the predictions, again demonstrating the influence of edge effects for smaller aspect ratio specimens. This effect can be very significantly reduced making wider specimens. This should be kept in mind while designing future hybrid octet specimens.

\subsection{Summary}

The measured strength and moduli of the hybrid octet structures are shown on a modified Ashby plot in Figure 8.16. It can be seen that the strength of these structures generally exceed those of similar hybrid CFRP-foam structures, but are also more dense. Octet hybrid structures can be manufactured as a compact, uniform material, not requiring face sheets. Thus, octet hybrid structures have the potential for a wider applications/uses than other hybrid structures. This chapter demonstrates that these octet hybrid structures can be readily designed and fabricated. 

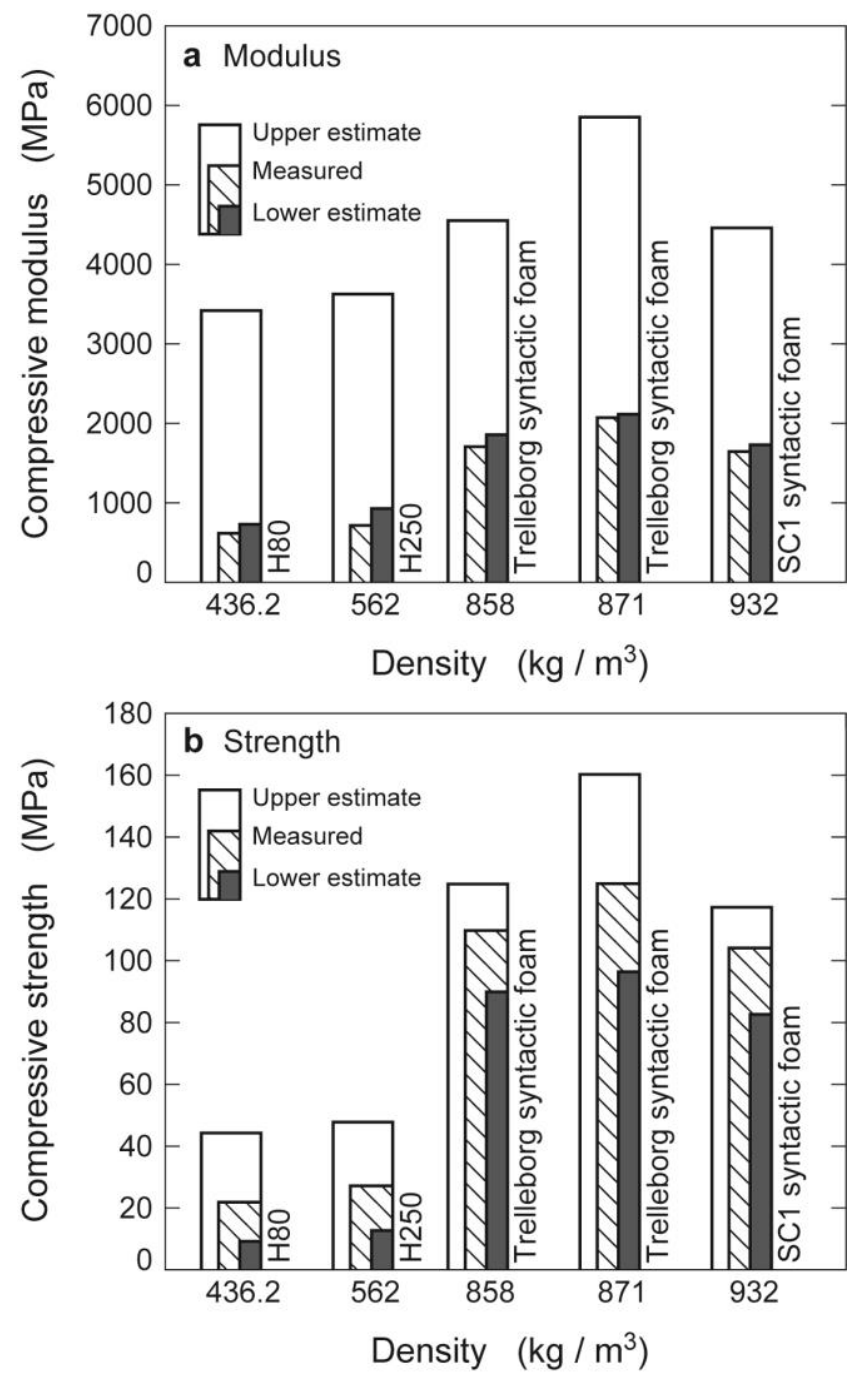

Figure 8.15: a) The upper and lower predicted modulus compared to the measured modulus. b) The upper and lower predicted collapse strength compared to the measured values. 

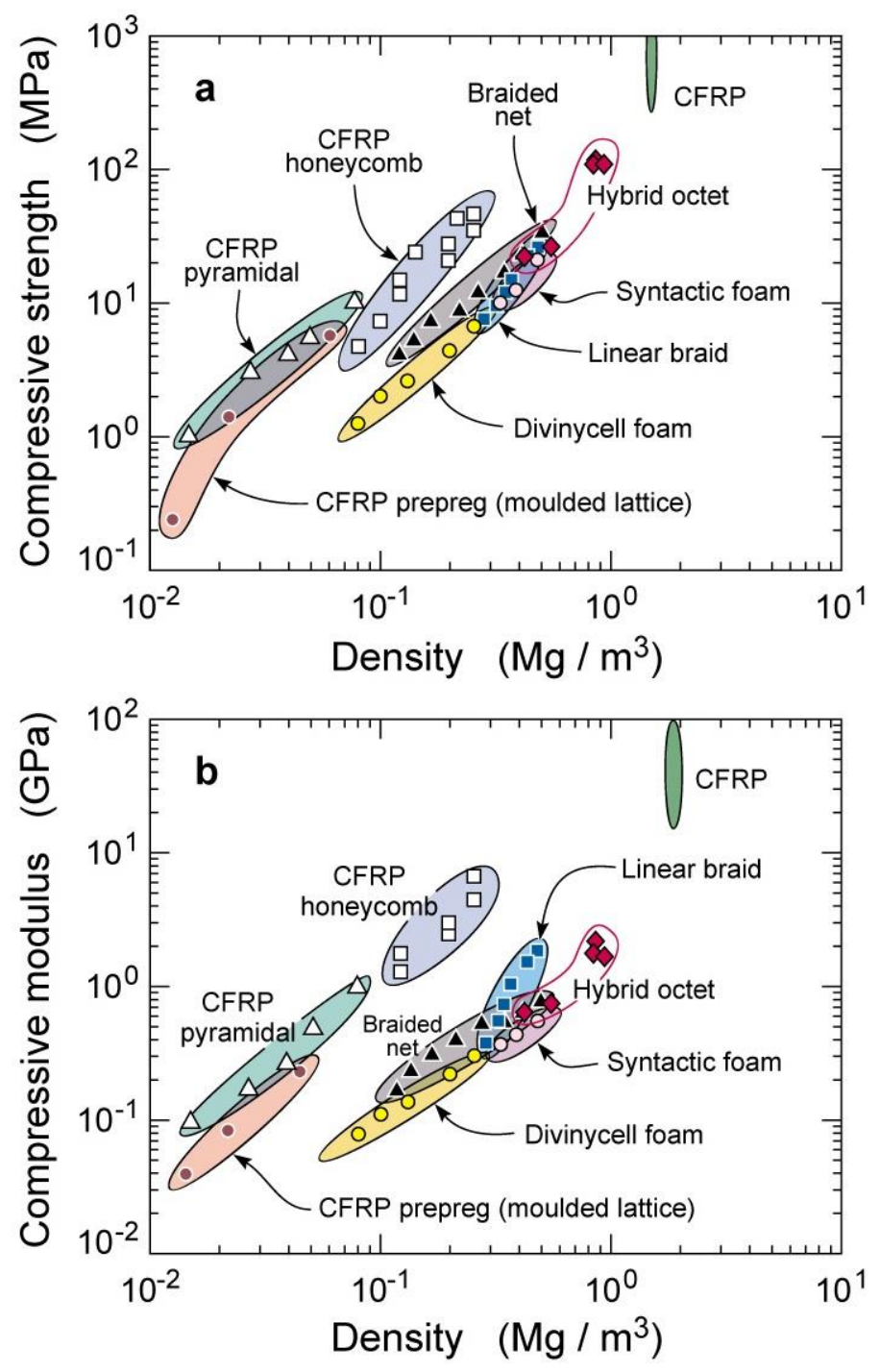

Figure 8.16: Modified Ashby charts showing the (a) compressive moduli and (b) compressive strength of the hybrid octet structures compared to other CFRP structures. 


\section{Chapter 9}

\section{Discussion}

This chapter examines the significance of the results discussed in the preceding chapters. This is considered in terms of strength, stiffness, and energy absorption. In addition, potential applications for the structures presented in this dissertation are discussed, as well as areas where further research is needed.

\subsection{Stiffness, strength, and energy absorption.}

Most sandwich panels are designed for stiffness, with sufficient strength to avoid failure. Other panels are designed to have high strength under loading. For still other applications, the panels are designed to have a significant amount of residual strength after initial strut failure. The results presented in this dissertation show that the use of carbon fiber composites advances the field of possibilities for each of these three applications.

\subsubsection{Stiffness}

A central motivation for the research presented in this dissertation is to create sandwich structures with high stiffness at a lower density, based on the use of high specific modulus CFRP materials. When designing sandwich panels for high stiffness, the modulus of the parent material used is critical, given that the modulus of the lattice would scale linearly with the modulus of the parent material for most periodic topologies (see Chapter 2). The selection of topology is also critical, due to the fact that some 
topologies (for example honeycombs) would have high stiffness for loading in compression, and much lower stiffness in shear or bending. The use of high specific modulus CFRP materials in fabricating the lattice, along with the selection of a pyramidal or octet topology, ensures the creation of a high stiffness lattice, with a good balance of properties in both through thickness compression and in-plane shear. This expectation is validated by the results presented in the preceding chapters. Figure 9.1 shows a modified Ashby chart, comparing the compressive and shear moduli of various competing lattice sandwich structure concepts. Also shown on the chart is the data for the lattices made from CFRP laminates, as well as the deduced empty lattice stiffness data for the pyramidal and octet lattice CFRP hybrids. It can be seen that the stiffness of the CFRP lattices are competitive with, or exceed those of competing concepts.

\subsubsection{Strength}

Another key motivation for the research presented in this dissertation is to create sandwich structures with high compressive and shear strengths at a lower density, through the use of high specific strength CFRP materials. The use of high specific strength CFRP materials in fabricating the lattice, as well as the pyramidal or octet topology, ensures the creation of a high strength lattice, with good strength in both compression and shear. The CFRP structures fabricated and tested, and analyzed as presented in Chapters 3-8 demonstrate that CFRP materials do indeed help fill in the existing gaps on the Ashby materials chart. Figure 9.2 shows a modified Ashby chart, comparing the compressive and shear strengths of various competing lattice sandwich structure concepts. Also shown on the chart is the data for the lattices made from CFRP laminates, as well as the deduced empty lattice strength data for the pyramidal and octet 
lattice CFRP hybrids. It can be seen that the compressive strength of the CFRP are competitive with, or exceed those of competing concepts. These results confirm that these structures offer an attractive alternative to metal lattices and foams for applications requiring high strength and low density. The empty lattice structures could be fabricated for more weight sensitive applications where residual strength and energy absorption are not critical factors, and the hybrid structures used for applications where the reverse is the case.

\subsubsection{Hybrid Core Design for Energy Absorption Applications.}

The main characteristics which dictate the energy absorption capacity for a cellular material to absorb energy include the plateau compressive stress, and the densification strain $\varepsilon_{d}$. Within a CFRP hybrid, the energy absorption capacity can be increased either by increasing the compressive plateau strength, or increasing the densification strain. Figure 9.3 shows the projected change in the energy absorption for the linear braid hybrid cores based on an increase in the truss inclination angle $\omega$ within the core. Increasing the truss inclination angle causes an increase in the compressive strength of the lattice (See Chapter 6), as well as a slight increase in the truss volume fraction within the hybrid core. The densification strain would increase slightly $\left(d_{l}\right.$ and $l$ are constant, $\sin \omega$ increases). 

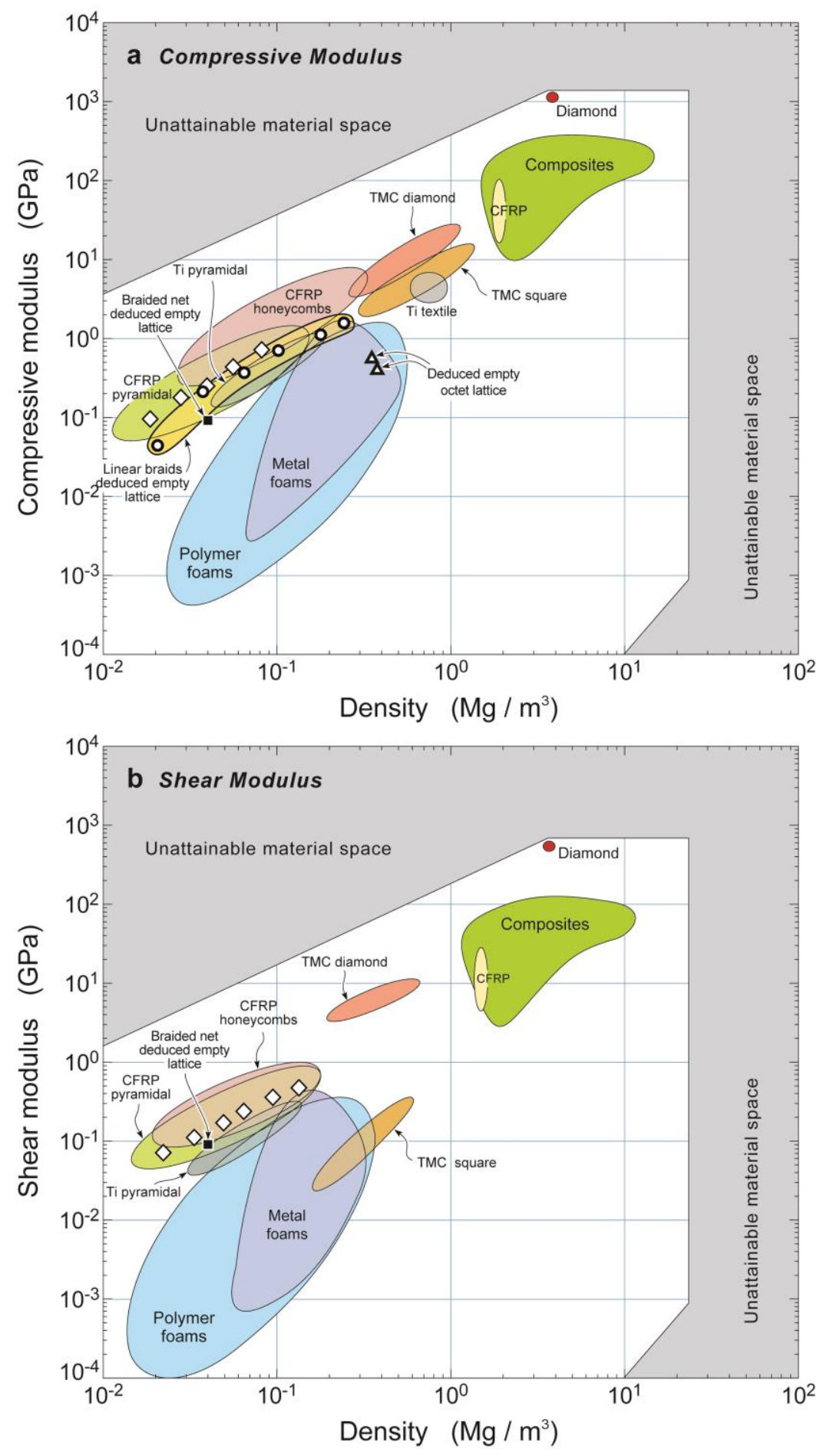

Figure 9.1: Modified Ashby ${ }^{10}$ charts showing the (a) compressive and (b) shear moduli of the structures developed in this dissertation, compared to other lattices/materials. 

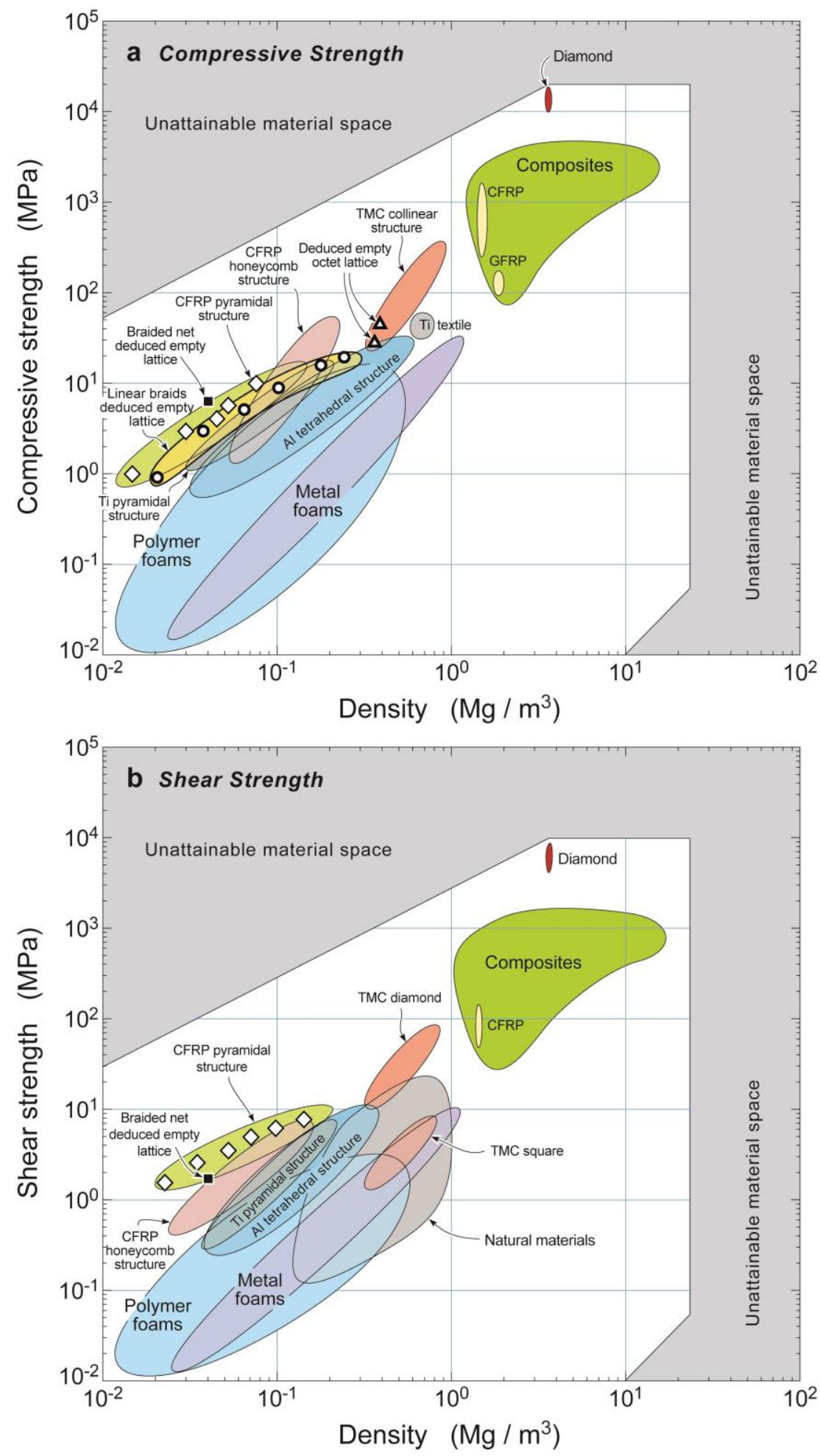

Figure 9.2: Modified Ashby ${ }^{10}$ charts showing the (a) compressive and (b) shear strengths of the structures developed in this dissertation, compared to other lattices/materials 
It can be seen that the linear braid structures would have approximately the same energy absorption capacity as the braided net structures for similar truss volume fractions and truss inclination angles within the hybrid. More importantly, hybrid cores can be designed to attain specific energy absorption capacities, depending on the requirements of the application. Core parameters such as truss inclination angle $\omega$, truss length $l$, truss diameter $d$, can be set based on what the desired energy absorption capacity of the finished hybrid would be. The capacity to set the fabrication parameters based on the final desired properties would be very useful for materials design and analysis.
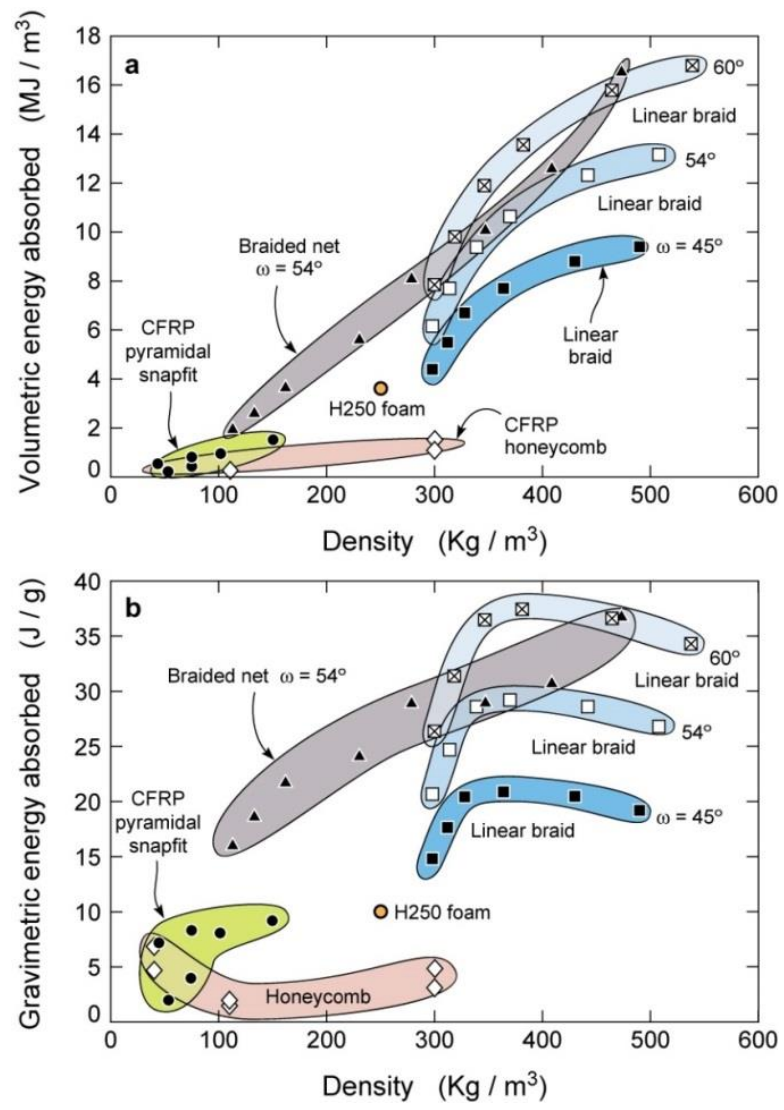

Figure 9.3: (a) Volumetric and (b) Gravimetric projections of the change in energy absorption of the linear braid hybrid cores for increases in the inclined strut angle $\omega$ within the hybrid. 


\subsection{Future Work}

\subsubsection{Application of fabrication methods to other composite materials}

There may exist materials with even higher stiffness and strength than CFRP composites from which lattice structures may be made. The basic fabrication approaches devised in this dissertation could be applied in the manufacture of such non-CFRP lattice structures. One potential area of application for these fabrication approaches is metal matrix composite structures. Nextel 610, for instance, is an alumina fiber which can be cast within aluminum alloy matrix to form a very high stiffness and strength composite. Nextel 610 in an $\mathrm{Al}_{2} \mathrm{Cu}$ matrix has been shown to have strengths of $3.4 \mathrm{GPa}$ in compression and 1.7 GPa in tension, and a Young's modulus of $240 \mathrm{GPa}^{68}$. A hybrid Nextel 610 metal matrix composite lattice could potentially be manufactured by utilizing the same principles used to make the linear braid hybrid structures. Preliminary experiments have shown that this concept is feasible. Figure 9.3 illustrates how the linear braid fabrication approach could be applied to making alumina fiber Nextel 610 metal matrix composite structures. The procedure would be as follows: prismatic mold pieces would be milled from blocks of a metal syntactic foam, replicating the shape of the desired unit cells, Figure 9.3a. Next, bundles of Nextel 610 fiber tows would be placed in the grooved channels, and the alternating mold pieces placed on top of the bundles to complete the assembly, such that the Nextel fiber bundles are completely contained within the channels created by the grooved molds, Figure $9.3 \mathrm{~b}$. Subsequently, layers of woven Nextel fabric would be placed on the top and bottom of the assembled part to complete the dry assembly, Figure 9.3c. The entire assembly is then placed in a pressure casting system, and infiltrated with liquid aluminum, or a liquid aluminum alloy to create 
the composite. The fabrication steps are similar to the linear braid fabrication procedure, and the materials are substituted and adapted for this application; for example, a metal foam is used instead of polymer foams or resin based syntactic foams; Nextel fiber is used instead of carbon fiber, and the infiltration is done using liquid aluminum instead of epoxy resin. By adapting the materials in a similar fashion, the techniques presented in this dissertation can potentially be applied to fabricating a wide range of composite structures.

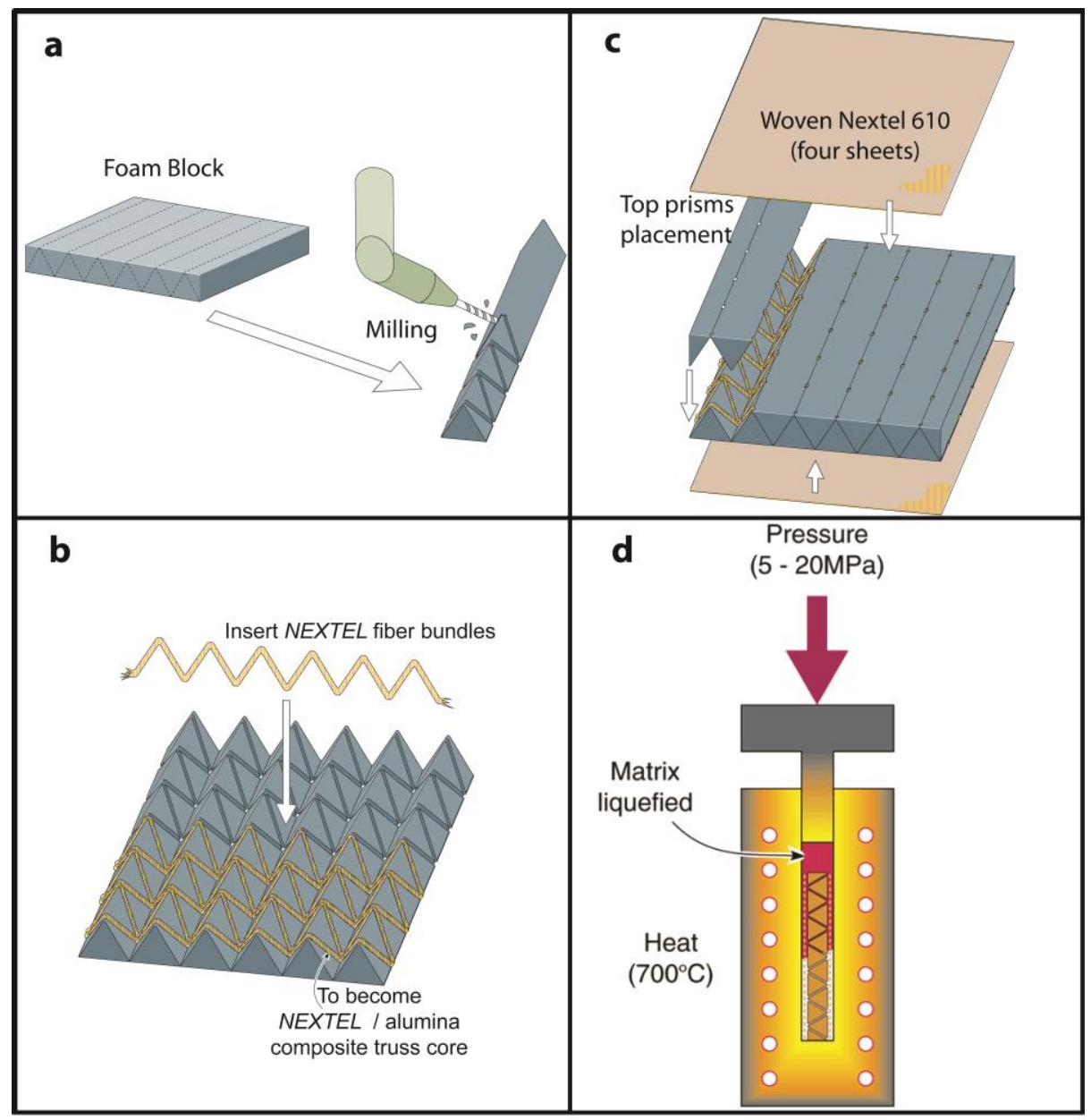

Figure 9.4: Proposed fabrication method for Nextel 610 metal matrix composites which mimics the linear braid fabrication procedure. 


\subsubsection{Further exploration of hybrid octet lattices}

One area for potential future work would be further exploration of the hybrid octet lattice structre. Given the potential advantages of this topology, it would be informative to characterize the change in mechanical behavior as the relative density of the truss material within the core changes. Also, given the brittle nature of most syntactic foams, hybrid carbon fiber octet lattices might be one potential method to enhance the fracture toughness of these foams. In addition, the behavior of these lattices under inplane shear loading and 3-point bending would provide clearer guidance on the utility of these structures for a wide variety of applications.

\subsubsection{Reduction of the the node saturation effect on core strength}

One effect that was consistently observed was that the presense of a node mass which did not contribute to core strength led to a saturation in strength as the truss density within the core increased, and the node volume became a larger fraction of the core. A minimum node mass was necessary to maintain arobust node/face sheet connection, and prevent node failure. Further exploration of alternative fabrication methods could yield ways to further minimize this effect. 


\section{Chapter 10}

\section{Conclusions}

Carbon fiber and carbon fiber hybrid sandwich structures have been fabricated using four different techniques, and their mechanical performance experimentally investigated. Micromechanical models have been developed which predict the behavior of these structures based on the parent material properties, unit cell topology, and core density. The predictions of these micromechanical models have been compared to the experimentally obtained results. It has been shown that:

1. Pyramidal truss sandwich cores with relative densities $\bar{\rho}$ in the range $1-10 \%$ have been manufactured from 0/90 crossply carbon fiber reinforced polymer laminates by employing a snap-fitting method.

2. The measured quasi-static shear strength of these laminated structures varied between 1 and 7.5 MPa, increasing with increasing $\bar{\rho}$.

3. Two failure modes were observed for the laminated structures: (i) Euler buckling of slender struts and (ii) delamination failure of stubby struts.

4. Analytical models are developed for the elastic response and collapse strengths of the laminated composite cores. In general good agreement between the measurements and predictions is obtained. 
5. A CFRP pyramidal lattice structure can be fabricated from a braided IM7 carbon fiber net using pre-machined polymer foam inserts and a pressure assisted SC 1 resin transfer molding process.

6. Sandwich panels with robustly bonded hybrid CRP/foam core 3D weave IM7 face sheets can be fabricated using Kevlar fiber reinforcement at the nodes.

7. The strength and moduli of the hybrid cores are found to increase with foam density due to a combination of increase in foam strength and modulus, and retention of a more circular cross section CFRP truss which is less susceptible to elastic buckling.

8. The moduli and strengths of the braided net hybrid cellular materials are well predicted by micromechanical models that decompose the applied stresses into axial and shear loads on the CFRP trusses and calculate the global (Euler) elastic and plastic microbuckling stresses for the struts.

9. The strength and moduli of the braided net hybrid material lie between those of foams and CFRP lattices, but their volumetric and gravimetric energy absorptions up to the onset of densification significantly exceed those of either material from which they are composed.

10. A hybrid CFRP sandwich structure can be manufactured using linear carbon fiber braids, woven CFRP face sheets, machined polymer foam inserts, and a vacuum assisted resin transfer molding process using SC1 epoxy.

11. The use of linear braids has facilitated the manufacture of hybrid cores with a with the truss volume fraction ranging from $1.5-17.5 \%$ of the core. 
12. The compressive strength and moduli of the linear braid hybrid cores increased with increasing truss volume fraction of the core, and eventually saturates due to an increasing portion of the truss volume being contained in the nodes.

13. The compressive strengths and moduli are well estimated by the braided net hybrid core micromechanical models which utilize parent material properties and unit cell geometry to predict core strength and moduli.

14. The strength of the linear braid hybrid structures are similar to that of the structures made using the braided net, and the modulus of the linear braid hybrids is higher for higher truss volume fractions. The energy absorption capacity of these structures is slightly less than those made using the braided net.

15. A hybrid CFRP octet structure can be fabricated using linear tows and pultruded rods, along with vacuum assisted resin transfer molding. This hybrid CFRP octet structure has cell size independent of face sheet separation. 


\section{References}

${ }^{1}$ Kooistra, G., Queheillalt, D., Wadley, H.N.G. Shear Behavior of Aluminum Lattice Truss Sandwich Panel Structures, Material Science and Engineering A 472 (2008) p 242250.

${ }^{2}$ Queheillalt, D.T., Wadley, H.N.G., Titanium alloy lattice truss structures. Materials and Design, 2009. 30: p. 1966-1975

${ }^{3}$ Cheung, K.C., Gershenfeld, N., Reversibly Assembled Cellular Composite Materials, Science, 341 (2013) p. 1219-1221.

${ }^{4}$ Finnegan, K., Kooistra, G., Wadley, H.N.G., The compressive response of carbon fiber composite pyramidal truss sandwich cores. International Journal of Materials Research, 2007. 98: p. 1264-1272.

5 Sypeck, D. J., Cellular Truss Core Sandwich Structures, Applied Composite Materials 12 (2005) p 229-246.

${ }^{6}$ Queheillalt, D.T., Murty, Y., Wadley, H.N.G., Mechanical properties of an extruded pyramidal lattice truss sandwich structure. Scripta Materialia 2008. 58: p. 76-79.

${ }^{7}$ Moongkhamklang, P., Elzey, D.M., Wadley, H.N.G., Titanium matrix composite lattice structures. Composites: Part A, 2008. 39: p. 176-187.

${ }^{8}$ Moongkhamklang, P., Deshpande, V.S., Wadley H.N.G, The compressive and shear response of titanium matrix composite lattice structures. Acta Materialia, 2010. 58: p. 2822-2835.

${ }^{9}$ Gibson, L.J., Ashby, M.F., Cellular Solids Structure and Properties. 1999: Cambridge University Press.

${ }^{10}$ Ashby M.F., Brechet Y.J.M., Designing hybrid materials, Acta Materiala , 51 (2003) p. 5801-5821.

${ }^{11}$ Picture credits: Sintered foam: http://www-diva.eng.cam.ac.uk/; Duocel foam: http://www.mdiproducts.com; Polyfoam: http://www.polyfoamcorp.com/.

12 Ashby, M.F., Metal foams: a design guide. 2000: Elsevier

${ }^{13}$ Mills N. Polymer foams handbook: engineering and biomechanics applications and design guide. Butterworth-Heinemann; 2007

${ }^{14}$ Penga, H.X., Fana, Z., Evans., J.R., Busfield, J.J., Microstructure of ceramic foams, Journal of the European Ceramic Society, 20 (2000) p. 807-813 
${ }^{15}$ Ashby, M.F., The properties of foams and lattices. Philosophical Transactions of the Royal Society A, 2006. 364(1838): p. 15-30.

${ }^{16}$ Wadley, H.N.G., Multifunctional periodic cellular metals. Transactions of the Royal Society A, 2006. 364(1838): p. 31-68.

${ }^{17}$ Evans, A.G., J.W. Hutchinson, and M.F. Ashby, Multifunctionality of cellular metal systems. Progress in Materials Science, 1999. 43, p. 171-221.

18 Deshpande, V. S., Ashby, M. F., and Fleck, N. A., Foam Topology Bending Versus Stretching Dominated Architectures, Acta Materialia. 49 (2001) p 1035-1040.

${ }^{19}$ Cote, F., Deshpande, V.S., Fleck, N. A., and Evans, A. G., The compressive and shear responses of corrugated and diamond lattice materials International Journal of Solids and Structures, 43 (2006) p 6220-6242.

${ }^{20}$ Bitzer, T., Honeycomb technology: materials, design, manufacturing, applications and testing. 1997: Kluwer Academic Pub.

${ }^{21}$ Cote, F., Deshpande, V. S., Fleck, N. A, and Evans, A. G., The Out-of-Plane Compressive Behavior of Metallic Honeycombs, Materials Science \& Engineering A, 380 (2004). p. 272-280.

${ }^{22}$ Paika, J.K., Thayamballi, A.K., Kima, G.S., The Strength Characteristics of Aluminum Honeycomb Sandwich Panels, Thin-Walled Structures 35 (1999) p 205-231.

${ }^{23}$ Sypeck, D.J, Wadley, H.N.G., Cellular Metal Truss Core Sandwich Structures, Advanced Engineering Materials, 10 (2002) p 759-764.

${ }^{24}$ Wadley, H.N.G., Fleck, N.A., Evans, A.G., Fabrication and Structural Performance of Periodic Cellular Metal Sandwich Structures, Composites Science and Technology 63 (2003) p 2331-2343.

${ }^{25}$ Queheillalt, D.T., Wadley, H.N.G., Pyramidal Lattice Truss Structures With Hollow Trusses Materials Science and Engineering A 397 (2005) p. 132-137.

${ }^{26}$ Russell, B.P., Deshpande, V.S., Wadley, H.N.G., Quasistatic deformation and failure modes of composite square honeycombs. Journal of Mechanics of Materials and Structures 2008. 3: p. 1315-1340.

${ }^{27}$ Deshpande, V.S., Fleck, N.A., Ashby, M.F., Effective properties of the octet-truss lattice material. Journal of the Mechanics and Physics of Solids 49 (8), 1747-1769

${ }^{28}$ Moongkhamklang, P., Titanium-Silicon Carbide Composite Lattice Structures, PhD Dissertation, University of Virginia, 2009. 
${ }^{29}$ Fleck N, An overview of the mechanical properties of foam and periodic lattice materials. Cellular Metals and Polymers 2004; p 3-7

${ }^{30}$ Deshpande VS, Fleck NA. Collapse of truss core sandwich beams in 3-point bending. International Journal of Solids and Structures 2001; 38, p 6275-6305.

${ }^{31} \mathrm{Lu}$ T. J., The effects of topology upon fluid-flow and heat-transfer within cellular copper structures. International Journal of Heat and Mass Transfer 2004; 47, p 31713186.

${ }^{32}$ Wang, B., Wu, L., Ma, L., Sun, Y., Du, S., Mechanical behavior of the sandwich structures with carbon fiber-reinforced pyramidal lattice truss core. Materials and Design, 2010. 31: p. 2659-2663.

${ }^{33}$ Xiong, J., Ma, L., Wu, L., Wang, B., Vaziri, A., Fabrication and crushing behavior of low density carbon fiber composite pyramidal truss structures. Composite Structures, 2010. 92: p. 2695-2702.

${ }^{34}$ George, T., Deshpande, V.S., Wadley, H.N.G., Mechanical response of carbon fiber composite sandwich panels with pyramidal truss cores. Composites Part A, 2013, 47.p. 31-40.

${ }^{35}$ Dharmasena, K.P., Wadley, H.N.G., Xue, W, and Hutchinson, J.W., Mechanical Response of Metallic Honeycomb Sandwich Panel Structures to High Intensity Dynamic Loading, International Journal of Impact Engineering, 35 (2008), p.1063-1074.

${ }^{36}$ Russell, B.P., Deshpande, V.S., Wadley, H.N.G., Quasistatic deformation and failure modes of composite square honeycombs. Journal of Mechanics of Materials and Structures 2008. 3: p. 1315-1340.

${ }^{37}$ Wen T., Tian J., Lu T.J., Queheillalt D.T., Wadley, H.N.G, Forced convection in metallic honeycomb structures, International Journal of Heat and Mass Transfer, 49 (2006), p.3313-3324.

${ }^{38}$ Gibson, L.J, Ashby, M.F., Schajer, G.S., Robertson, C.I., The mechanics of Two Dimensional Cellular Materials, The Proceedings of the Royal Society A, 382 (1982), p. 25-42.

${ }^{39}$ Gibson, L.J., Ashby, M.F., Cellular Solids Structure and Properties. 1999: Cambridge University Press.

${ }^{40}$ Kooistra, G.W., Deshpande, V.S., Wadley, H.N.G, Hierarchical Corrugated Core Sandwich Panel Concepts, Journal of Applied Mechanics, 74 (2007) p 259-268. 
${ }^{41}$ Rimoli, J.J.,Talamini, B., Wetzel, J.J., Dharmasena, K.P, Radovitzky, R., Wadley, H.N.G, Wet-sand impulse loading of metallic plates and corrugated core sandwich panels, International Journal of Impact Engineering, 38 (2011), p 837-848.

${ }^{42}$ Rathbun, H.J., Wei, Z., He, M.Y., Zok, F.W., Evans, A.G., Sypeck, D.J., Wadley, H.N.G., Measurement and Simulation of the Performance of a Lightweight Metallic Sandwich Structure with a Tetrahedral Truss Core, Journal of Applied Mechanics, 71 (2004), p. 368-374.

${ }^{43}$ Kooistra G.W., Deshpande VS, and Wadley HNG. Compressive behavior of age hardenable tetrahedral lattice truss structures made from aluminum. Acta Materialia 2004; 52, p 4229-4237.

${ }^{44}$ Chiras S., Mumm, D.R., Evans, A.G., Wicks, N., Hutchinson, J.W., Dharmasena, K., Wadley, H.N.G., Fichter, S., The structural performance of near-optimized truss core panels, International Journal of Solids and Structures, 39 (2002), p. 4093-4115.

${ }^{45}$ Deshpande V.S, Fleck N.A, Collapse of truss core sandwich beams in 3-point bending, International Journal of Solids and Structures 2001; 38, p 6275-6305.

${ }^{46}$ ASTM D6641 / D6641M Standard Test Method for Determining the Compressive Properties of Polymer Matrix Composite Laminates Using a Combined Loading Compression (CLC) Test Fixture.

${ }^{47}$ ASTM C273 / C273M Standard Test Method for Shear Properties of Sandwich Core Materials.

${ }^{48}$ Spathis G, Kontou E. Non-linear viscoplastic behavior of fiber reinforced polymer composites, Composites Science and Technology 2004; 64; p. 2333-2340.

${ }^{49}$ Argon A. Fracture of composites. Treatise of Material Science and Technology 1972; 1, p. 79-114.

${ }^{50}$ Budiansky B, Fleck NA. Compressive failure of fiber composites, Journal of the Mechanics and Physics of Solids 1993; 41, p.183-211.

${ }^{51}$ Yurgatis SW. Measurement of small angle misalignments in continuous fibre composites. Composites Science and Technology 1987; 30, p. 279-293.

${ }^{52}$ Ko, F.K., Preform Architecture for Ceramic-Matrix Composites. American Ceramic Society Bulletin, 1989. 68(2): p. 401-414.

${ }^{53}$ Ko, F.K., Engineered Materials Handbook. Vol. 1. 1987, Metals Park, Ohio: ASM International. 
${ }^{54}$ Bogdanovich, A., Mungalov, D., Recent Advancements in Manufacturing 3-D Braided Preforms and Composites. Proc. of Int. Conf. ACUN-4, 2002: p. 61-72.

${ }^{55}$ Mungalov, D., Bogdanovich, A, Complex Shape 3-D Braided Composite Preforms: Structural Shapes for Marine and Aerospace. SAMPE Journal, 2004. 40(3): p. 7-20.

${ }^{56}$ Kostar, T.D., Chou, T.-W, Braided Structures, 3-D Textile Reinforcements in Composite Materials ed. A. Miravete. Cambridge, England: Woodhead Publ. Ltd.

${ }^{57}$ Mungalov, D., Bogdanovich, A, Automated 3-D Braiding Machine and Method, United States Patent No. 6439096, Issued August 2002.

${ }^{58}$ Odom, E. M, Adams, D. F, Failure modes of unidirectional carbon/epoxy composite compression specimens, Journal of Composites. 1990, 21(4), p 289 - 296.

${ }^{59}$ Queheillalt, D.T., Deshpande, V.S., Wadley, H.N.G., Truss Waviness Effects in Cellular Lattice Structures, Journal of Mechanics of Materials and Structures, 2 (2007), p. $1657-1775$.

${ }^{60} \mathrm{Li}$, Q. M, Magkiriadis, I and Harrigan, J.J. Compressive Strain at the Onset of Densification of Cellular Solids, Journal of Cellular Plastics, 2006, 42 p 371- 391.

${ }^{61}$ Queheillalt, D.T., Wadley, H.N.G., Cellular metal lattices with hollow trusses, Acta Materialia, 53 (2005), p. 303-313.

${ }^{62}$ Odom, E. M, Adams, D. F, Failure modes of unidirectional carbon/epoxy composite compression specimens, Journal of Composites. 1990, 21(4), p $289-296$.

${ }^{63}$ Cox, B.N., Dadkhah, M.S., Morris, W.L., Tensile failure of 3D woven composites, Composites, Part A, 27 (1996), p. 447-458.

${ }^{64}$ Fleck, N.A., Jelf, P.M., Compression Failure Mechanisms in Unidirectional Composites, Journal of Composite Materials, 26 (1992), p. 2706-2726.

${ }^{65}$ Cartie, D.D., Fleck, N.A., The effect of pin reinforcement upon the through-thickness compressive strength of foam-cored sandwich panels, Composites Science and Technology, 63 (2003), p. 2401-2409.

${ }^{66}$ Mouritz, A.P., Review of z-pinned composite laminates, Composites: Part A, 38 (2007), p. 2383-2397.

${ }^{67}$ A.J. Malcom, M.T. Aronson, V.S. Deshpande, H.N.G. Wadley, Compressive Response of Glass Fiber Composite Sandwich Structures, Composites: Part A, 54, p. 88-97. 
${ }^{68}$ Aluminum Matrix Composites, Typical Properties Data sheet, AMCPROP No. 1 Rev 3, 3M Corporation, St Paul, MN. 